\title{
Photoassisted Diversity-Oriented Synthesis: Intramolecular Cycloadditions of Photogenerated Azaxylylenes with Oxazole Pendants, and Subsequent Postphotochemical Multicomponent Modifications.
}

\author{
N.N. Bhuvan Kumar and Andrei G. Kutateladze* \\ Department of Chemistry and Biochemistry, University of Denver, Denver, CO 80208
}

\section{SUPPORTING INFORMATION}

\section{Table of contents}

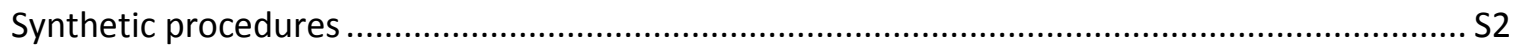

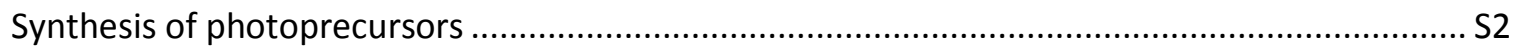

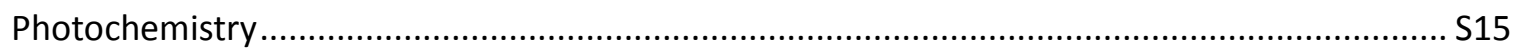

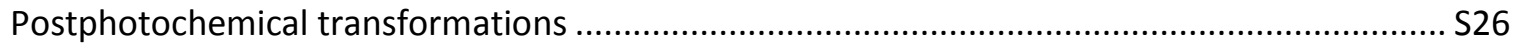

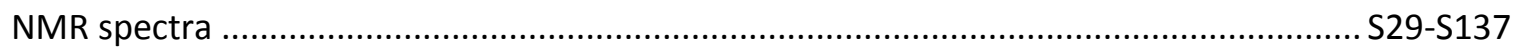

(cif files for $\mathbf{x}$-ray structures of compounds $\mathbf{3 c}$, 11f, and $\mathbf{1 5}$ are uploaded separately)

Abbreviations:

HATU: (1-[Bis(dimethylamino)methylene]-1H-1,2,3-triazolo[4,5-b]pyridinium 3-oxid hexafluorophosphate); HOAT: 1-Hydroxy-7-azabenzotriazole; DIPEA: di-isopropyl-ethylamine; DMP: Dess-Martin periodinane 


\section{SYNTHETIC PROCEDURES}

Common solvents were purchased from Pharmco and used as is, except for THF, which was refluxed over and distilled from potassium benzophenone ketyl prior to use. Common reagents were purchased from Aldrich or TCI America and used without additional purification, unless indicated otherwise. NMR spectra were recorded at $25^{\circ} \mathrm{C}$ on a Bruker Avance III $500 \mathrm{MHz}$ in $\mathrm{CDCl}_{3}$ with TMS as an internal standard (unless noted otherwise). X-Ray structures were obtained with a Bruker APEX II instrument. High resolution mass spectra were obtained on the $M D S$ SCIEX/Applied Biosystems API QSTARTM Pulsar i Hybrid LC/MS/MS System mass spectrometer at CU-Boulder. Flash column chromatography was performed using Teledyne Ultra Pure Silica Gel (230 - 400 mesh) on a Teledyne Isco Combiflash Rf.

\section{SYNTHESIS OF PHOTOPRECUSORS}<smiles>Nc1ccccc1C1OCCO1</smiles>

N-(2-(1,3-Dioxolan-2-yl)phenylamine: $1.00 \mathrm{~g}$ (1 eq, $5.13 \mathrm{mmol})$ of 1-(1,3dioxolan-2-yl)-2-nitrobenzene in $10 \mathrm{~mL}$ of ethanol was added to a stirred suspension of $\mathrm{Na}_{2} \mathrm{~S}(2.5$ eq, $0.86 \mathrm{~g}, 11.1 \mathrm{mmol})$ in ethanol $(25 \mathrm{~mL})$. The mixture was refluxed for 1 hour, then TEA $(0.5$ $\mathrm{mL})$ was added and the mixture was concentrated. The residue was then dissolved in $\mathrm{Et}_{2} \mathrm{O}(20 \mathrm{~mL})$, TEA $(0.5 \mathrm{~mL}), \mathrm{H}_{2} \mathrm{O}(10 \mathrm{~mL})$. The aqueous phase was extracted with $\mathrm{Et}_{2} \mathrm{O}(3 \times 20 \mathrm{~mL})$, the combined organic phases were washed with $\mathrm{H}_{2} \mathrm{O}(20 \mathrm{~mL})$, dried over $\mathrm{Na}_{2} \mathrm{SO}_{4}$ and concentrated to yield 0.47 $\mathrm{g}(55 \%)$ of the product. ${ }^{1} \mathrm{H} \mathrm{NMR}\left(500 \mathrm{MHz}, \mathrm{CDCl}_{3}\right) \delta 7.36(\mathrm{t}, J=7.8,1 \mathrm{H}), 7.19(\mathrm{td}, J=7.8,1.5$, $1 \mathrm{H}), 6.79(\mathrm{td}, J=7.6,0.9,1 \mathrm{H}), 6.70(\mathrm{dd}, J=8.0,0.6,1 \mathrm{H}), 5.85(\mathrm{~s}, 1 \mathrm{H}), 4.23(\mathrm{~s}, 2 \mathrm{H}), 4.13(\mathrm{~m}, 2 \mathrm{H})$, 4.06 (m, 2H). ${ }^{13} \mathrm{C} \mathrm{NMR}\left(126 \mathrm{MHz}, \mathrm{CDCl}_{3}\right) \delta 145.4,130.0,127.5,121.3,117.9,116.5,103.3,64.9$.

General Procedure A for the synthesis of amido-4-oxopentanoates (S1a-e):<smiles>COC(=O)CCC(=O)CCC(=O)CNC(=O)CCC(=O)OC</smiles> 
5-aminolevulinic acid methyl ester hydrochloride $(3 \mathrm{mmol})$, triethylamine $(9 \mathrm{mmol})$ were taken up in dichloromethane $(40 \mathrm{~mL})$ and treated with acyl chloride $(3.60 \mathrm{mmol})$ at $0{ }^{\circ} \mathrm{C}$ under nitrogen atmosphere. The obtained mixture was stirred at room temperature overnight. The reaction mixture was washed with $20 \mathrm{~mL}$ of water. Solvent was removed under reduced pressure and the residue was purified by flash column chromatography on silica gel to give amido-4-oxopentanoates.<smiles>COC(=O)CCC(=O)CNC(=O)c1ccccc1</smiles>

Methyl 5-benzamido-4-oxopentanoate (S1a): General procedure A was followed using 5-aminolevulinic acid methyl ester hydrochloride (2.01 g, $11.07 \mathrm{mmol})$, benzoyl chloride ( $1.87 \mathrm{~g}, 13.28 \mathrm{mmol}, 1.54 \mathrm{~mL})$ and triethylamine $(3.36 \mathrm{~g}, 33.20 \mathrm{mmol}, 4.63 \mathrm{~mL})$ in $\mathrm{CH}_{2} \mathrm{Cl}_{2}(100 \mathrm{~mL})$. After purification by flash column chromatography $2.51 \mathrm{~g}(91 \%)$ of product S1a was obtained. ${ }^{1} \mathrm{H}$ NMR $\left(500 \mathrm{MHz}, \mathrm{CDCl}_{3}\right) \delta 7.78-7.70(\mathrm{~m}, 2 \mathrm{H}), 7.43-7.33(\mathrm{~m}, 2 \mathrm{H}), 7.29$ (t, $J=7.7 \mathrm{~Hz}, 2 \mathrm{H}), 4.21(\mathrm{~d}, J=5.1 \mathrm{~Hz}, 2 \mathrm{H}), 3.54(\mathrm{~s}, 3 \mathrm{H}), 2.68(\mathrm{t}, J=6.5 \mathrm{~Hz}, 2 \mathrm{H}), 2.53(\mathrm{~d}, J=12.9$ $\mathrm{Hz}, 2 \mathrm{H}) .{ }^{13} \mathrm{C} \mathrm{NMR}\left(126 \mathrm{MHz}, \mathrm{CDCl}_{3}\right) \delta 204.39,172.78,167.34,133.50,131.52,128.34,126.97$, $51.66,49.34,34.32,27.37$.

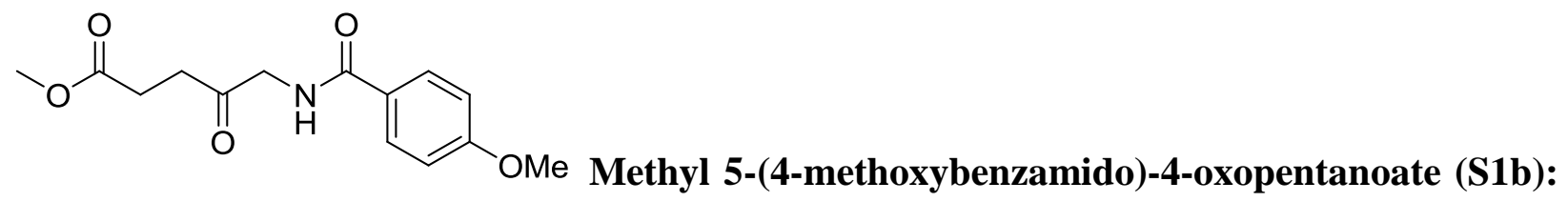

General procedure $\mathbf{A}$ was followed using 5-aminolevulinic acid methyl ester hydrochloride ( 0.52 g, $2.86 \mathrm{mmol}), p$-anisoyl chloride $(0.58 \mathrm{~g}, 3.43 \mathrm{mmol}, 0.46 \mathrm{~mL})$ and triethylamine $(0.87 \mathrm{~g}, 8.59$ $\mathrm{mmol}, 1.20 \mathrm{~mL})$ in $\mathrm{CH}_{2} \mathrm{Cl}_{2}(40 \mathrm{~mL})$. After purification by flash column chromatography $0.66 \mathrm{~g}$ (83\%) of product $\mathbf{S 1 b}$ was obtained. ${ }^{1} \mathrm{H}$ NMR $\left(500 \mathrm{MHz}, \mathrm{CDCl}_{3}\right) \delta 7.77(\mathrm{~d}, J=8.90 \mathrm{~Hz}, 2 \mathrm{H}), 6.91$ (d, $J=8.90 \mathrm{~Hz}, 2 \mathrm{H}), 6.88$ (br, 1H), 4.38 (d, $J=4.6 \mathrm{~Hz}, 2 \mathrm{H}), 3.84$ (s, 3H), 3.68 (s, 3H), $2.85-2.76$ (m, 2H), $2.68(\mathrm{dd}, J=7.4,5.4 \mathrm{~Hz}, 2 \mathrm{H}) .{ }^{13} \mathrm{C}$ NMR $\left(126 \mathrm{MHz}, \mathrm{CDCl}_{3}\right) \delta 204.32,172.96,166.88$, 162.52, 129.03, 126.13, 113.91, 55.53, 52.09, 49.79, 34.79, 27.76. 
<smiles>COC(=O)CCC(=O)CNC(=O)c1ccco1</smiles>

\section{Methyl 5-(furan-2-carboxamido)-4-oxopentanoate (S1c):}

General procedure A was followed using 5-aminolevulinic acid methyl ester hydrochloride (0.80 g, $4.40 \mathrm{mmol})$, 2-Furoyl chloride (0.69 g, $5.28 \mathrm{mmol}, 0.52 \mathrm{ml})$ and triethylamine $(1.34 \mathrm{~g}, 13.21$ $\mathrm{mmol}, 1.84 \mathrm{~mL})$ in $\mathrm{CH}_{2} \mathrm{Cl}_{2}(50 \mathrm{~mL})$. After purification by flash column chromatography $0.99 \mathrm{~g}$ $(94 \%)$ of the product (S1c) was obtained. ${ }^{1} \mathrm{H} \mathrm{NMR}\left(500 \mathrm{MHz}, \mathrm{CDCl}_{3}\right) \delta 7.43(\mathrm{dd}, J=1.8,0.8 \mathrm{~Hz}$, 1H), 7.11 (br, 1H), 7.08 (dd, $J=3.5,0.8 \mathrm{~Hz}, 1 \mathrm{H}), 6.46$ (dd, $J=3.5,1.8 \mathrm{~Hz}, 1 \mathrm{H}), 4.33$ (d, $J=5.0$ $\mathrm{Hz}, 2 \mathrm{H}), 3.64(\mathrm{~s}, 3 \mathrm{H}), 2.77(\mathrm{dd}, J=7.5,5.6 \mathrm{~Hz}, 2 \mathrm{H}), 2.65(\mathrm{dd}, J=7.4,5.4 \mathrm{~Hz}, 2 \mathrm{H}) .{ }^{13} \mathrm{C}$ NMR $(126$ $\left.\mathrm{MHz}, \mathrm{CDCl}_{3}\right) \delta 203.73,172.88,158.29,147.50,144.36,114.66,112.16,51.99,48.83,34.65$, 27.67.<smiles>COC(=O)CCC(=O)CNC(=O)c1cccs1</smiles>

Methyl 4-oxo-5-(thiophene-2-carboxamido)pentanoate (S1d):

General procedure A was followed using 5-aminolevulinic acid methyl ester hydrochloride (1.03 $\mathrm{g}, 5.67 \mathrm{mmol})$, 2-thiophenecarbonyl chloride $(0.99 \mathrm{~g}, 6.80 \mathrm{mmol}, 0.73 \mathrm{ml})$ and triethylamine $(1.72$ g, $17.01 \mathrm{mmol}, 2.37 \mathrm{~mL})$ in $\mathrm{CH}_{2} \mathrm{Cl}_{2}(50 \mathrm{~mL})$. After purification by flash column chromatography $1.29 \mathrm{~g}(89 \%)$ of product S1d was obtained. ${ }^{1} \mathrm{H} \mathrm{NMR}\left(500 \mathrm{MHz}, \mathrm{CDCl}_{3}\right) \delta 7.54(\mathrm{dd}, J=3.8,1.2$ $\mathrm{Hz}, 1 \mathrm{H}), 7.42(\mathrm{dd}, J=5.0,1.2 \mathrm{~Hz}, 1 \mathrm{H}), 7.20(\mathrm{t}, J=5.0 \mathrm{~Hz}, 1 \mathrm{H}), 7.02-6.96(\mathrm{~m}, 1 \mathrm{H}), 4.26(\mathrm{~d}, J=$ $5.0 \mathrm{~Hz}, 2 \mathrm{H}), 3.60(\mathrm{~s}, 3 \mathrm{H}), 2.74(\mathrm{dd}, J=7.4,5.5 \mathrm{~Hz}, 2 \mathrm{H}), 2.59(\mathrm{dd}, J=7.3,5.5 \mathrm{~Hz}, 2 \mathrm{H}) .{ }^{13} \mathrm{C} \mathrm{NMR}$ $\left(126 \mathrm{MHz} \mathrm{CDCl}_{3}\right) \delta 204.42,172.97,162.03,138.11,130.46,128.60,127.72,51.88,49.35,34.48$, 27.54 .<smiles>COC(=O)CCC(=O)CNC(=O)c1cnco1</smiles>

Methyl 5-(oxazole-5-carboxamido)-4-oxopentanoate (S1e):

General procedure A was followed using 5-aminolevulinic acid methyl ester hydrochloride (0.49 $\mathrm{g}, 2.71 \mathrm{mmol})$, and triethylamine $(1.37 \mathrm{~g}, 13.55 \mathrm{mmol}, 1.89 \mathrm{~mL})$ in $\mathrm{CH}_{2} \mathrm{Cl}_{2}(25 \mathrm{~mL})$. The reaction mixture was treated with oxazole-5-carbonyl chloride $(2.98 \mathrm{mmol})$ in $5 \mathrm{~mL}$ of $\mathrm{CH}_{2} \mathrm{Cl}_{2}$ at $0^{\circ} \mathrm{C}$ under 
nitrogen atmosphere. After purification by flash column chromatography $0.44 \mathrm{~g}(68 \%)$ of product S1e was obtained. ${ }^{1} \mathrm{H}$ NMR $\left(500 \mathrm{MHz}, \mathrm{CDCl}_{3}\right) \delta 7.92$ (s, 1H), 7.72 (s, 1H), 7.08 (br, 1H), 4.37 (d, $J=4.8 \mathrm{~Hz}, 2 \mathrm{H}), 3.66(\mathrm{~s}, 3 \mathrm{H}), 2.83-2.73(\mathrm{~m}, 2 \mathrm{H}), 2.73-2.63(\mathrm{~m}, 2 \mathrm{H}) .{ }^{13} \mathrm{C}$ NMR $(126 \mathrm{MHz}$, $\left.\mathrm{CDCl}_{3}\right) \delta 203.26,172.89,156.72,151.68,145.15,130.73,52.12,48.88,34.72,27.75$.

General Procedure B for the synthesis of oxazole propanoates (S2a-e): Burgess reagent (3.0 to $6.0 \mathrm{mmol}$ ) was added to a solution of 5-(aroylamido)levulinic acid methyl ester $\mathbf{S 1}$ (2.0 mmol) and triethylamine $(2.4 \mathrm{mmol})$ in acetonitrile $(15 \mathrm{~mL})$ and refluxed until the starting material was consumed. Solvent was removed under reduced pressure and the residue was purified by flash column chromatography on silica gel to yield desired oxazole propanoates.
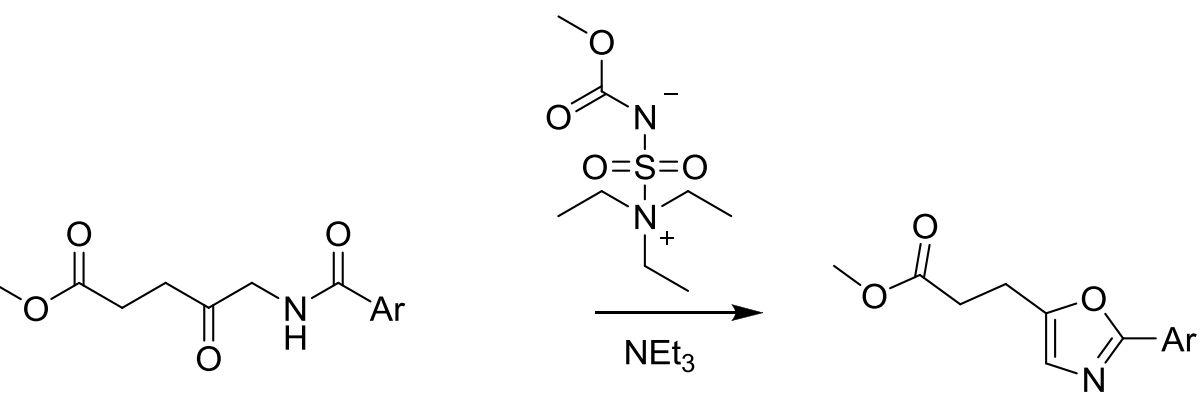<smiles>COC(=O)CCc1cnc(-c2ccccc2)o1</smiles>

Methyl 3-(2-phenyloxazol-5-yl)propanoate (S2a): General procedure B was followed using 5-benzamido-4-oxopentanoate $(2.51 \mathrm{~g}, 10.06 \mathrm{mmol})$, Burgess reagent $(3.39 \mathrm{~g}, 15.10 \mathrm{mmol})$ and triethylamine $(1.32 \mathrm{~g}, 13.08 \mathrm{mmol}, 1.83 \mathrm{~mL})$ in acetonitrile $(50$ $\mathrm{mL})$. After purification by flash column chromatography $2.11 \mathrm{~g}(91 \%)$ of product S2a was obtained. ${ }^{1} \mathrm{H}$ NMR $\left(500 \mathrm{MHz}, \mathrm{CDCl}_{3}\right) \delta 8.02-7.97(\mathrm{~m}, 2 \mathrm{H}), 7.47-7.41(\mathrm{~m}, 3 \mathrm{H}), 6.89$ (t, $J=1.1$ $\mathrm{Hz}, 1 \mathrm{H}), 3.72(\mathrm{~s}, 3 \mathrm{H}), 3.08$ (ddd, $J=7.8,7.2,1.1 \mathrm{~Hz}, 2 \mathrm{H}), 2.74(\mathrm{dd}, J=8.1,7.0 \mathrm{~Hz}, 2 \mathrm{H}) .{ }^{13} \mathrm{C} \mathrm{NMR}$ $\left(126 \mathrm{MHz}, \mathrm{CDCl}_{3}\right) \delta 172.32,160.92,150.96,130.01,128.68,127.57,125.98,124.13,51.81$, $32.05,21.19$. 


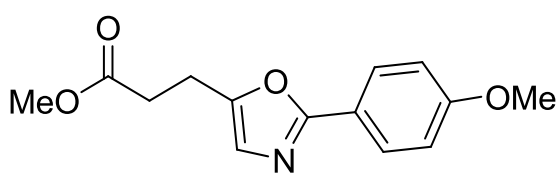

\section{Methyl 3-(2-(4-methoxyphenyl)oxazol-5-yl)propanoate}

(S2b): General procedure B was followed using 5-(4-methoxybenzoyl)aminolevulinic acid methyl ester $(0.66 \mathrm{~g}, 2.36 \mathrm{mmol})$, Burgess reagent $(0.84 \mathrm{~g}, 3.54 \mathrm{mmol})$ and triethylamine $(0.31 \mathrm{~g}, 3.07$ $\mathrm{mmol}, 0.43 \mathrm{~mL})$ in acetonitrile $(15 \mathrm{~mL})$. After purification by flash column chromatography 0.59 g $(96 \%)$ of product $\mathbf{S 2 b}$ was obtained. ${ }^{1} \mathrm{H}$ NMR $\left(500 \mathrm{MHz}, \mathrm{CDCl}_{3}\right) \delta 7.91(\mathrm{~d}, J=8.90 \mathrm{~Hz}, 2 \mathrm{H})$, $6.94(\mathrm{~d}, J=9.0 \mathrm{~Hz}, 2 \mathrm{H}), 6.82(\mathrm{t}, J=1.0 \mathrm{~Hz}, 1 \mathrm{H}), 3.83(\mathrm{~s}, 3 \mathrm{H}), 3.70(\mathrm{~s}, 3 \mathrm{H}), 3.04(\mathrm{~m}, 2 \mathrm{H}), 2.70(\mathrm{~m}$, 2H). ${ }^{13} \mathrm{C}$ NMR $\left(126 \mathrm{MHz}, \mathrm{CDCl}_{3}\right) \delta 172.56,161.22,161.18,150.45,127.78,123.96,120.59$, 114.24, 55.46, 51.96, 32.29, 21.34.<smiles>COC(=O)CCc1cnc(-c2cccs2)o1</smiles>

Methyl 3-(2-(thiophen-2-yl)oxazol-5-yl)propanoate (S2c): General

procedure B was followed using methyl 4-oxo-5-(thiophene-2-carboxamido)pentanoate $(0.52 \mathrm{~g}$, $2.04 \mathrm{mmol})$, Burgess reagent $(0.73 \mathrm{~g}, 3.06 \mathrm{mmol})$ and triethylamine $(0.27 \mathrm{~g}, 2.65 \mathrm{mmol}, 0.37 \mathrm{~mL})$ in acetonitrile $(15 \mathrm{~mL})$. After purification by flash column chromatography $0.45 \mathrm{~g}(93 \%)$ of product S2c was obtained. ${ }^{1} \mathrm{H}$ NMR $\left(500 \mathrm{MHz}, \mathrm{CDCl}_{3}\right) \delta 7.56(\mathrm{dd}, J=3.7,1.2 \mathrm{~Hz}, 1 \mathrm{H}), 7.35(\mathrm{dd}$, $J=5.0,1.2 \mathrm{~Hz}, 1 \mathrm{H}), 7.05(\mathrm{dd}, J=5.0,3.7 \mathrm{~Hz}, 1 \mathrm{H}), 6.80(\mathrm{t}, J=1.1 \mathrm{~Hz}, 1 \mathrm{H}), 3.67(\mathrm{~s}, 3 \mathrm{H}), 3.01(\mathrm{td}$, $J=7.5,1.2 \mathrm{~Hz}, 2 \mathrm{H}), 2.67(\mathrm{dd}, J=8.1,6.9 \mathrm{~Hz}, 2 \mathrm{H}) .{ }^{13} \mathrm{C} \mathrm{NMR}\left(126 \mathrm{MHz}, \mathrm{CDCl}_{3}\right) \delta 172.34,157.20$, $150.56,130.18,127.88,127.86,127.18,124.07,51.90,32.10,21.16$.

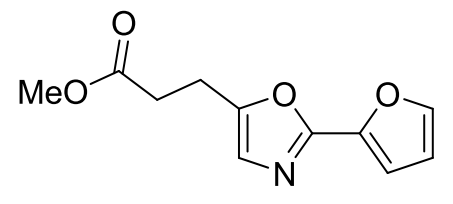

Methyl 3-(2-(furan-2-yl)oxazol-5-yl)propanoate (S2d): General procedure B was followed using methyl 4-oxo-5-(furan-2-carboxamido)pentanoate (0.99 g, 4.14 $\mathrm{mmol})$, Burgess reagent $(4.48 \mathrm{~g}, 18.63 \mathrm{mmol})$ and triethylamine $(0.54 \mathrm{~g}, 5.38 \mathrm{mmol}, 0.75 \mathrm{~mL})$ in acetonitrile $(20 \mathrm{~mL})$. After purification by flash column chromatography $0.56 \mathrm{~g}(61 \%)$ of product S2d was obtained. ${ }^{1} \mathrm{H}$ NMR $\left(500 \mathrm{MHz}, \mathrm{CDCl}_{3}\right) \delta 7.51(\mathrm{dd}, J=1.8,0.8 \mathrm{~Hz}, 1 \mathrm{H}), 6.93(\mathrm{dd}, J=3.5$, $0.8 \mathrm{~Hz}, 1 \mathrm{H}), 6.85(\mathrm{t}, J=1.1 \mathrm{~Hz}, 1 \mathrm{H}), 6.50(\mathrm{dd}, J=3.5,1.8 \mathrm{~Hz}, 1 \mathrm{H}), 3.69(\mathrm{~s}, 3 \mathrm{H}), 3.04(\mathrm{td}, J=7.5$, $1.1 \mathrm{~Hz}, 2 \mathrm{H}), 2.69(\mathrm{dd}, J=8.0,7.0 \mathrm{~Hz}, 3 \mathrm{H}) .{ }^{13} \mathrm{C} \mathrm{NMR}\left(126 \mathrm{MHz}, \mathrm{CDCl}_{3}\right) \delta 172.39,153.95,150.65$, $144.19,143.15,124.12,111.83,110.90,51.99,32.13,21.19$. 
<smiles>COC(=O)CCc1cnc(-c2cnco2)o1</smiles>

Methyl 3-(2,5'-bioxazol-5-yl)propanoate (S2e): General procedure B was followed using methyl 5-(oxazole-5-carboxamido)-4-oxopentanoate (0.44 g, $1.83 \mathrm{mmol})$, Burgess reagent $(0.65 \mathrm{~g}, 2.74 \mathrm{mmol})$ and triethylamine $(0.24 \mathrm{~g}, 2.38 \mathrm{mmol}, 0.33 \mathrm{~mL})$ in acetonitrile $(15 \mathrm{~mL})$. After purification by flash column chromatography $0.21 \mathrm{~g}(51 \%)$ of product S2e was obtained. ${ }^{1} \mathrm{H}$ NMR $\left(500 \mathrm{MHz}, \mathrm{CDCl}_{3}\right) \delta 7.98(\mathrm{~s}, 1 \mathrm{H}), 7.62(\mathrm{~s}, 1 \mathrm{H}), 6.95(\mathrm{t}, J=1.1 \mathrm{~Hz}, 1 \mathrm{H}), 3.71$ (s, 3H), $3.08(\mathrm{td}, J=7.5,1.1 \mathrm{~Hz}, 2 \mathrm{H}), 2.73(\mathrm{t}, J=7.5 \mathrm{~Hz}, 2 \mathrm{H}) .{ }^{13} \mathrm{C} \mathrm{NMR}\left(126 \mathrm{MHz}, \mathrm{CDCl}_{3}\right) \delta$ 172.29, 151.90, 151.65 (two signals overlap), 141.12, 126.80, 124.68, 52.12, 32.05, 21.21.

\section{General Procedure C for hydrolysis of methyl esters S2 to oxazolylpropanoic acids (1a-e):}

A $1 \mathrm{M}$ sodium hydroxide aqueous solution $(7 \mathrm{~mL}, 7 \mathrm{mmol})$ was added to a solution of oxazole propanoates $(2 \mathrm{mmol})$ in methanol $(10 \mathrm{~mL})$ and the obtained mixture was then stirred at room temperature for 3 hours. Thereafter, $1 \mathrm{M}$ hydrochloric acid was added to the reaction solution until the reaction mixture reaches $\mathrm{pH} 4$ and the generated solid was then collected by filtration, washed with water and then washed with a mixture of $n$-hexane and dichloromethane $20 \mathrm{~mL}(4: 1)$, so as to obtain the title compound.
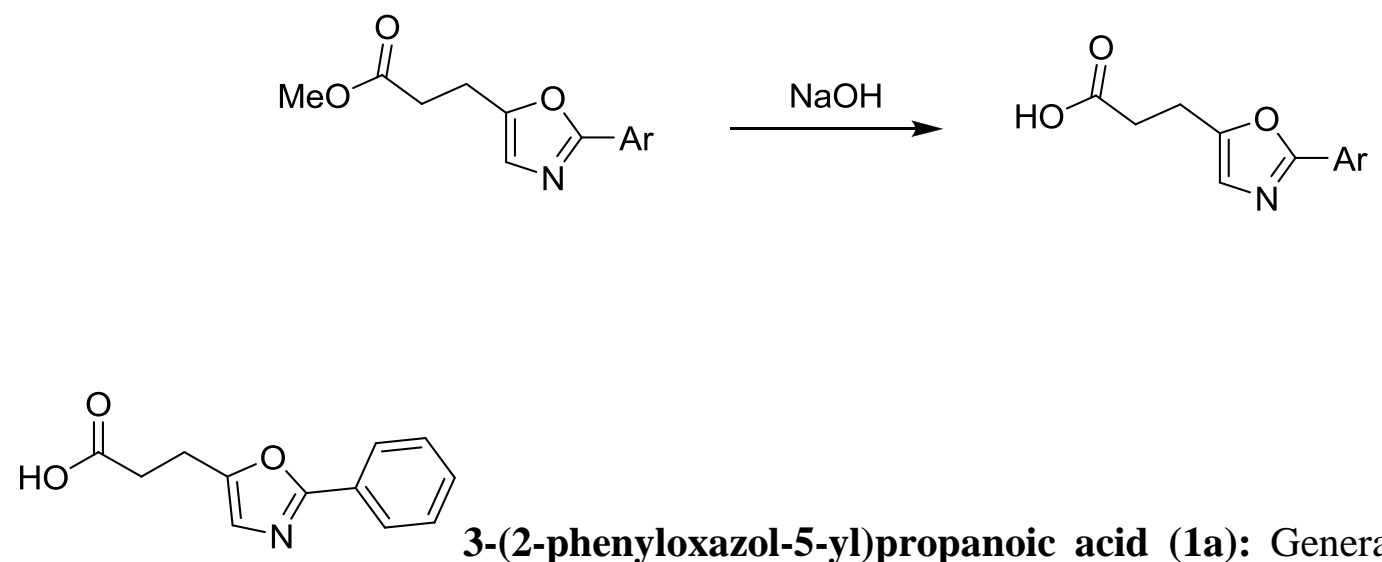

3-(2-phenyloxazol-5-yl)propanoic acid (1a): General procedure C was followed using methyl 3-(2-phenyloxazol-5-yl)propanoate $(2.11 \mathrm{~g}, 9.07 \mathrm{mmol})$ in $75 \mathrm{~mL}$ of methanol and $1 \mathrm{M}$ aqueous sodium hydroxide $(27 \mathrm{~mL})$, which gave $1.76 \mathrm{~g}$, (89\%) of 1a. ${ }^{1} \mathrm{H}$ NMR $\left(500 \mathrm{MHz} \mathrm{CDCl}_{3}\right) \delta 7.92(\mathrm{~d}, J=9.0 \mathrm{~Hz}, 2 \mathrm{H}), 6.96(\mathrm{~d}, J=9.0 \mathrm{~Hz}, 2 \mathrm{H}), 6.89$ (t, $\left.J=1.0 \mathrm{~Hz}, 2 \mathrm{H}\right)$, 3.85 (s, 3H), 3.07 (m, 2H), 2.78 (m, 2H). ${ }^{13} \mathrm{C}$ NMR (126 MHz, $\left.\mathrm{CDCl}_{3}\right) \delta 175.83,161.38,161.34$, $150.31,127.93,123.95,120.42,114.33,55.53,32.01,21.19$. 
<smiles>COc1ccc(-c2ncc(CCC(=O)O)o2)cc1</smiles>

3-(2-(4-methoxyphenyl)oxazol-5-yl)propanoic acid (1b):

General procedure C was followed using methyl 3-(2-(4-methoxyphenyl)oxazol-5-yl)propanoate $(0.59 \mathrm{~g}, 2.11 \mathrm{mmol})$ in $10 \mathrm{~mL}$ of methanol and $1 \mathrm{M}$ aqueous sodium hydroxide $(7 \mathrm{~mL})$, which gave $0.45 \mathrm{~g},(86 \%)$ of $\mathbf{1 b} .{ }^{1} \mathrm{H}$ NMR $\left(500 \mathrm{MHz}, \mathrm{CDCl}_{3}\right) \delta 7.92(\mathrm{~d}, J=9.0 \mathrm{~Hz}, 2 \mathrm{H}), 6.96(\mathrm{~d}, J=9.0 \mathrm{~Hz}$, $2 \mathrm{H}), 6.89(\mathrm{t}, J=1.0 \mathrm{~Hz}, 2 \mathrm{H}), 3.85(\mathrm{~s}, 3 \mathrm{H}), 3.07(\mathrm{~m}, 2 \mathrm{H}), 2.78(\mathrm{~m}, 2 \mathrm{H}) .{ }^{13} \mathrm{C} \mathrm{NMR}\left(126 \mathrm{MHz}, \mathrm{CDCl}_{3}\right)$ $\delta 175.83,161.38,161.34,150.31,127.93,123.95,120.42,114.33,55.53,32.01,21.19$.<smiles>O=C(O)CCc1cnc(-c2cccs2)o1</smiles>

3-(2-(thiophen-2-yl)oxazol-5-yl)propanoic acid (1c): General procedure $\mathrm{C}$ was followed using methyl 3-(2-(thiophen-2-yl)oxazol-5-yl)propanoate (0.45 g, 1.90 $\mathrm{mmol})$ in $10 \mathrm{~mL}$ of methanol and $1 \mathrm{M}$ aqueous sodium hydroxide $(5.7 \mathrm{~mL})$, which gave $0.41 \mathrm{~g}(97$ \%) of 1c. ${ }^{1} \mathrm{H}$ NMR (500 MHz, CD $\left.{ }_{3} \mathrm{OD}\right) \delta 7.67(\mathrm{~m}, 1 \mathrm{H}), 7.60(\mathrm{~m}, 1 \mathrm{H}), 7.17(\mathrm{~m}, 1 \mathrm{H}), 6.91(\mathrm{~s}, 1 \mathrm{H})$, $3.04(\mathrm{t}, J=7.3 \mathrm{~Hz}, 2 \mathrm{H}), 2.71(\mathrm{t}, J=7.3 \mathrm{~Hz}, 2 \mathrm{H}) .{ }^{13} \mathrm{C} \mathrm{NMR}\left(126 \mathrm{MHz}, \mathrm{CD}_{3} \mathrm{OD}\right) \delta 175.79,158.66$, $153.11,130.36,129.83,129.18,128.97,124.29,32.79,21.94$.<smiles>O=C(O)CCc1cnc(-c2ccco2)o1</smiles>

3-(2-(furan-2-yl)oxazol-5-yl)propanoic acid (1d): General procedure $\mathrm{C}$ was followed using methyl 3-(2-(furan-2-yl)oxazol-5-yl)propanoate (0.56 g, $2.53 \mathrm{mmol})$ in $15 \mathrm{~mL}$ of methanol and $1 \mathrm{M}$ aqueous sodium hydroxide $(7.5 \mathrm{~mL})$, which gave $0.45 \mathrm{~g}(86 \%) \mathbf{1 d}$. ${ }^{1} \mathrm{H}$ NMR $\left(500 \mathrm{MHz}, \mathrm{CD}_{3} \mathrm{OD}\right) \delta 7.69(\mathrm{dd}, J=1.8,0.8 \mathrm{~Hz}, 1 \mathrm{H}), 7.04(\mathrm{dd}, J=3.4,0.8 \mathrm{~Hz}, 1 \mathrm{H}), 6.93$ $(\mathrm{t}, J=1.1 \mathrm{~Hz}, 1 \mathrm{H}), 6.61(\mathrm{dd}, J=3.5,1.8 \mathrm{~Hz}, 1 \mathrm{H}), 3.01(\mathrm{ddd}, J=7.9,7.2,1.1 \mathrm{~Hz}, 2 \mathrm{H}), 2.53(\mathrm{dd}, J$ $=8.1,7.1 \mathrm{~Hz}, 2 \mathrm{H}) .{ }^{13} \mathrm{C} \mathrm{NMR}\left(126 \mathrm{MHz}, \mathrm{CD}_{3} \mathrm{OD}\right) \delta 180.20,155.09,154.69,145.98,143.98$, 123.69, 112.90, 112.32, 36.54, 23.54. 
<smiles>O=C(O)CCc1cnc(-c2cnco2)o1</smiles>

3-(2,5'-bioxazol-5-yl)propanoic acid (1e): General procedure C was followed using methyl 3-(2,5'-bioxazol-5-yl)propanoate $(0.21 \mathrm{~g}, 0.94 \mathrm{mmol})$ in $5 \mathrm{~mL}$ of methanol and $1 \mathrm{M}$ aqueous sodium hydroxide $(3 \mathrm{~mL})$, which gave $0.16 \mathrm{~g}(82 \%)$ of 1 e. ${ }^{1} \mathrm{H}$ NMR $(500 \mathrm{MHz}$, $\left.\mathrm{CD}_{3} \mathrm{OD}\right) \delta 8.36(\mathrm{~s}, 1 \mathrm{H}), 7.73(\mathrm{~s}, 1 \mathrm{H}), 7.02(\mathrm{t}, J=1.1 \mathrm{~Hz}, 1 \mathrm{H}), 3.06-3.01(\mathrm{~m}, 2 \mathrm{H}), 2.54(\mathrm{t}, J=7.5$ $\mathrm{Hz}, 2 \mathrm{H}) .{ }^{13} \mathrm{C} \mathrm{NMR}\left(126 \mathrm{MHz}, \mathrm{CD}_{3} \mathrm{OD}\right) \delta 180.27,170.65,155.87,154.30,152.49,142.11,127.32$, $124.42,36.33,23.46$.

General procedure D for the synthesis of photoprecursors 2a-e (i.e. 2-aryloxazol-5-yl) and 9a-c (i.e. 5-aryloxazol-2-yl):<smiles>Nc1ccccc1C1OCCO1</smiles><smiles>[R]c1ncc(CCC(=O)O)o1</smiles>

(1) HATU, HOAT DIPEA

(2) PTSA. $\mathrm{H}_{2} \mathrm{O}$<smiles>[R]c1ncc(CCC(=O)Nc2ccccc2C=O)o1</smiles>

2a-e, $35 \%-85 \%$ $\mathrm{R}=\mathrm{Ph}, \mathrm{p}$-anisyl, 2-thienyl, 2-furyl, 2-oxazolyl

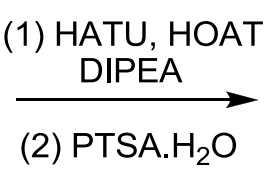

9a-c, $55 \%-89 \%$ $\mathrm{R}=\mathrm{Ph}, 4-\mathrm{Cl}-\mathrm{Phenyl}$, 4-Br-Phenyl

Aryloxazolyl propanoic acid (5.0 mmol), HATU (6.0 mmol), HOAt (6.0 mmol), and DIPEA (12.5 mmol) were dissolved in $12 \mathrm{~mL}$ of DMF and stirred for $5 \mathrm{~min}$ at room temperature. To this reaction mixture 2-(1,3-dioxolan-2-yl)aniline $(6.0 \mathrm{mmol})$ was added and allowed to stir for $48 \mathrm{~h}$. The solvent was removed under vacuum. The residue was dissolved in $150 \mathrm{~mL}$ of $\mathrm{CH}_{2} \mathrm{Cl}_{2}$ and washed with $50 \mathrm{~mL}$ of $5 \% \mathrm{NaHCO}_{3}$ and $50 \mathrm{~mL}$ of water. The organic layer was dried over $\mathrm{Na}_{2} \mathrm{SO}_{4}$. The solvent was removed under reduced pressure and the residue was purified by flash column 
chromatography. The resulting acetal was deprotected by stirring with $\mathrm{TsOH} \cdot \mathrm{H}_{2} \mathrm{O}(1.0 \mathrm{mmol}, 20$ $\mathrm{mol} \%$ ) in $30 \mathrm{~mL}$ of acetone at $20^{\circ} \mathrm{C}$ for $24 \mathrm{~h}$. The photoprecursors 1a-e and $\mathbf{9 f}-\mathbf{h}$ were purified by flash column chromatography on silica gel.

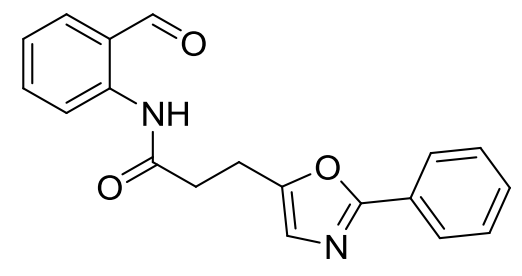

N-(2-formylphenyl)-3-(2-phenyloxazol-5-yl)propanamide (2a): General procedure D was followed using 3-(2-(phenyl)oxazol-5-yl)propanoic acid (0.89 g, 4.09 mmol), HATU (1.87 g, $4.92 \mathrm{mmol})$, HOAT (0.67 g, $4.92 \mathrm{mmol})$, DIPEA (1.32 g, $10.02 \mathrm{mmol}$, $1.78 \mathrm{~mL})$ and 2-(1,3-dioxolan-2-yl)aniline $(0.81 \mathrm{~g}, 4.92 \mathrm{mmol})$ in $15 \mathrm{~mL}$ of DMF. The product was deprotected in $20 \mathrm{~mL}$ of acetone with $\mathrm{TsOH} \cdot \mathrm{H}_{2} \mathrm{O}(0.16 \mathrm{~g}, 0.82 \mathrm{mmol})$. Purification by flash column chromatography gave $1.12 \mathrm{~g}(85 \%)$ of $\mathbf{2 a} .{ }^{1} \mathrm{H}$ NMR $\left(500 \mathrm{MHz}, \mathrm{CDCl}_{3}\right) \delta 11.23(\mathrm{~s}, 1 \mathrm{H})$, $9.88(\mathrm{~d}, J=0.7 \mathrm{~Hz}, 1 \mathrm{H}), 8.74(\mathrm{~d}, J=8.4 \mathrm{~Hz}, 1 \mathrm{H}), 8.01-7.94(\mathrm{~m}, 2 \mathrm{H}), 7.65(\mathrm{dd}, J=7.7,1.6 \mathrm{~Hz}$, 1H), 7.61 (ddd, $J=8.7,7.3,1.7 \mathrm{~Hz}, 1 \mathrm{H}), 7.44-7.37$ (m, 3H), $7.22(\mathrm{td}, J=7.5,1.1 \mathrm{~Hz}, 1 \mathrm{H}), 6.93$ (s, $1 \mathrm{H}), 3.20$ (ddd, $J=7.8,6.8,1.1 \mathrm{~Hz}, 2 \mathrm{H}), 2.87$ (dd, $J=8.2,6.8 \mathrm{~Hz}, 2 \mathrm{H}) .{ }^{13} \mathrm{C}$ NMR $(126 \mathrm{MHz}$, $\left.\mathrm{CDCl}_{3}\right) \delta 195.70,170.58,161.13,151.05,140.77,136.35,136.16,130.12,128.78,127.68,126.17$, 124.44, 123.19, 121.67, 120.02, 36.15, 21.50. HRMS (ESI) calcd for $\mathrm{C}_{19} \mathrm{H}_{17} \mathrm{~N}_{2} \mathrm{O}_{3}\left(\mathrm{MH}^{+}\right)$ 321.1233 , found 321.1228 .

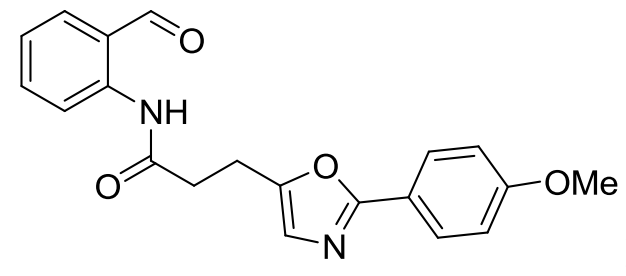

\section{N-(2-formylphenyl)-3-(2-(4-methoxyphenyl)oxazol-5-}

yl)propanamide (2b): General procedure D was followed using 3-(2-(4-methoxyphenyl)oxazol5-yl)propanoic acid (0.27 g, $1.09 \mathrm{mmol})$, HATU (0.49 g, $1.31 \mathrm{mmol})$, HOAT (0.18 g, $1.31 \mathrm{mmol})$, DIPEA (0.35 g, $2.72 \mathrm{mmol}, 0.47 \mathrm{~mL})$ and 2-(1,3-dioxolan-2-yl)aniline $(0.22 \mathrm{~g}, 1.31 \mathrm{mmol})$ in 5 $\mathrm{mL}$ of DMF. The product was deprotected in $20 \mathrm{~mL}$ of acetone with $\mathrm{TsOH} \cdot \mathrm{H}_{2} \mathrm{O}(0.04 \mathrm{~g}, 0.22$ mmol). Purification by flash column chromatography gave $0.26 \mathrm{~g}(68 \%)$ of $\mathbf{2 b} .{ }^{1} \mathrm{H}$ NMR (500 $\left.\mathrm{MHz}, \mathrm{CDCl}_{3}\right) \delta 11.24(\mathrm{~s}, 1 \mathrm{H}), 9.89(\mathrm{~d}, J=0.7 \mathrm{~Hz}, 1 \mathrm{H}), 8.75(\mathrm{~d}, J=8.4 \mathrm{~Hz}, 1 \mathrm{H}), 7.91(\mathrm{~d}, J=9.0$ 
$\mathrm{Hz}, 2 \mathrm{H}), 7.67(\mathrm{dd}, J=7.6,1.6 \mathrm{~Hz}, 1 \mathrm{H}), 7.65-7.60(\mathrm{~m}, 1 \mathrm{H}), 7.24(\mathrm{td}, J=7.5,1.0 \mathrm{~Hz}, 1 \mathrm{H}), 6.93$ $(\mathrm{d}, J=9.0 \mathrm{~Hz}, 2 \mathrm{H}), 6.90-6.87(\mathrm{~m}, 1 \mathrm{H}), 3.85(\mathrm{~s}, 3 \mathrm{H}), 3.18(\mathrm{ddd}, J=7.9,6.7,1.1 \mathrm{~Hz}, 2 \mathrm{H}), 2.87$ $(\mathrm{dd}, J=8.2,6.8 \mathrm{~Hz}, 2 \mathrm{H}) .{ }^{13} \mathrm{C}$ NMR $\left(126 \mathrm{MHz}, \mathrm{CDCl}_{3}\right) \delta 195.74,170.71,161.27,161.22,150.45$, $140.84,136.40,136.20,127.86,124.19,123.22$, 121.72, 120.63, 120.08, 114.24, 55.50, 36.29, 21.55. HRMS (ESI) calcd for $\mathrm{C}_{20} \mathrm{H}_{19} \mathrm{~N}_{2} \mathrm{O}_{4}\left(\mathrm{MH}^{+}\right) 351.1339$, found 351.1338 .

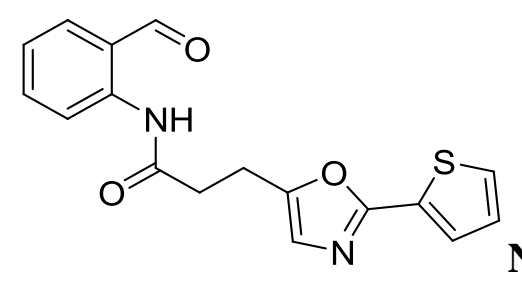

\section{N-(2-formylphenyl)-3-(2-(thiophen-2-yl)oxazol-5-yl)}

propanamide (2c): General procedure D was followed using 3-(2-(thiophen-2-yl)oxazol-5yl)propanoic acid (0.25 g, $1.12 \mathrm{mmol})$, HATU (0.51 g, $1.34 \mathrm{mmol})$, HOAT (0.18 g, $1.34 \mathrm{mmol})$, DIPEA (0.36 g, $2.80 \mathrm{mmol}, 0.49 \mathrm{~mL})$ and 2-(1,3-dioxolan-2-yl)aniline $(0.22 \mathrm{~g}, 1.34 \mathrm{mmol})$ in 5 $\mathrm{mL}$ of DMF. The product was deprotected in $20 \mathrm{~mL}$ of acetone with $\mathrm{TsOH} \cdot \mathrm{H}_{2} \mathrm{O}(0.04 \mathrm{~g}, 0.82$ mmol). Purification by flash column chromatography gave $0.23 \mathrm{~g}(63 \%)$ of $2 \mathrm{c} .{ }^{1} \mathrm{H}$ NMR (500 $\left.\mathrm{MHz}, \mathrm{CDCl}_{3}\right) \delta 11.24(\mathrm{~s}, 1 \mathrm{H}), 9.91(\mathrm{~s}, 1 \mathrm{H}), 8.75(\mathrm{~d}, J=8.4 \mathrm{~Hz}, 1 \mathrm{H}), 7.68(\mathrm{dd}, J=7.6,1.6 \mathrm{~Hz}, 1 \mathrm{H})$, 7.63 (dddd, $J=8.5,7.4,1.7,0.5 \mathrm{~Hz}, 1 \mathrm{H}), 7.60(\mathrm{dd}, J=3.7,1.2 \mathrm{~Hz}, 1 \mathrm{H}), 7.38(\mathrm{dd}, J=5.0,1.2 \mathrm{~Hz}$, $1 \mathrm{H}), 7.24(\mathrm{dd}, J=7.5,1.1 \mathrm{~Hz}, 1 \mathrm{H}), 7.08(\mathrm{dd}, J=5.0,3.7 \mathrm{~Hz}, 1 \mathrm{H}), 6.90-6.87(\mathrm{~m}, 1 \mathrm{H}), 3.18(\mathrm{~m}$, 2H), $2.87(\mathrm{~m}, 2 \mathrm{H}) .{ }^{13} \mathrm{C} \mathrm{NMR}\left(126 \mathrm{MHz}, \mathrm{CDCl}_{3}\right) \delta 195.77,170.55,157.42,150.67,140.83,136.43$, 136.21, 130.36, 127.97, 127.36, 124.42, 123.27, 121.75, 120.11, 36.17, 21.46. HRMS (ESI) calcd for $\mathrm{C}_{17} \mathrm{H}_{15} \mathrm{~N}_{2} \mathrm{O}_{3} \mathrm{~S}\left(\mathrm{MH}^{+}\right)$327.0798, found 327.0797.

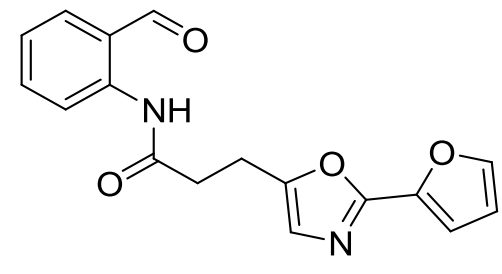

\section{N-(2-formylphenyl)-3-(2-(furan-2-yl)oxazol-5-yl)propanamide}

(2d): General procedure D was followed using3-(2-(furan-2-yl)oxazol-5-yl)propanoic acid (0.20 g, $0.96 \mathrm{mmol})$, HATU (0.44 g, $1.15 \mathrm{mmol})$, HOAT (0.16 g, $1.15 \mathrm{mmol})$, DIPEA (0.31 g, 2.40 $\mathrm{mmol}, 0.42 \mathrm{~mL})$ and 2-(1,3-dioxolan-2-yl)aniline $(0.19 \mathrm{~g}, 1.15 \mathrm{mmol})$ in $4 \mathrm{~mL}$ of DMF. The 
product was deprotected in $8 \mathrm{~mL}$ of acetone with $\mathrm{TsOH} \cdot \mathrm{H}_{2} \mathrm{O}(0.04 \mathrm{~g}, 0.19 \mathrm{mmol})$ stirring for $24 \mathrm{~h}$ at room temperature. Purification by flash column chromatography gave $0.15 \mathrm{~g}(50 \%)$ of $\mathbf{2 d} .{ }^{1} \mathrm{H}$ NMR (500 MHz, $\left.\mathrm{CHCl}_{3}\right) \delta 11.22(\mathrm{~s}, 1 \mathrm{H}), 9.90(\mathrm{~d}, J=0.7 \mathrm{~Hz}, 1 \mathrm{H}), 8.73(\mathrm{~d}, J=8.4 \mathrm{~Hz}, 1 \mathrm{H}), 7.67$ (dd, $J=7.6,1.6 \mathrm{~Hz}, 1 \mathrm{H}), 7.64-7.59$ (m, 1H), 7.52 (dd, $J=1.7,0.8 \mathrm{~Hz}, 1 \mathrm{H}), 7.24$ (td, $J=7.5,1.0$ $\mathrm{Hz}, 1 \mathrm{H}), 6.95(\mathrm{dd}, J=3.4,0.8 \mathrm{~Hz}, 1 \mathrm{H}), 6.91(\mathrm{t}, J=1.1 \mathrm{~Hz}, 1 \mathrm{H}), 6.51(\mathrm{dd}, J=3.5,1.8 \mathrm{~Hz}, 1 \mathrm{H})$, $3.18(\mathrm{ddd}, J=8.8,6.6,1.1 \mathrm{~Hz}, 2 \mathrm{H}), 2.87(\mathrm{dd}, J=8.2,6.8 \mathrm{~Hz}, 2 \mathrm{H}) .{ }^{13} \mathrm{C} \mathrm{NMR}\left(126 \mathrm{MHz}, \mathrm{CDCl}_{3}\right)$ $\delta$ 195.74, 170.47, 154.03, 150.68, 144.20, 143.20, 140.79, 136.40, 136.19, 124.35, 123.25, 121.72, 120.07, 111.85, 110.96, 36.05, 21.35. HRMS (ESI) calcd for $\mathrm{C}_{17} \mathrm{H}_{15} \mathrm{~N}_{2} \mathrm{O}_{4}\left(\mathrm{MH}^{+}\right) 311.1026$, found 311.1031.

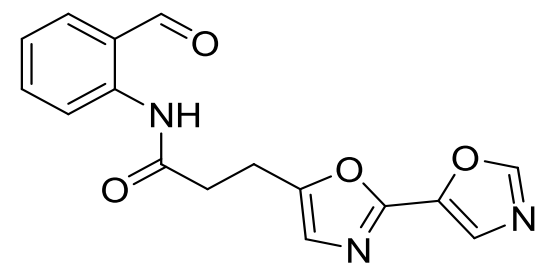

3-(2,5'-bioxazol-5-yl)-N-(2-formylphenyl)propanamide (2e): General procedure D was followed using 3-(2,5'-bioxazol-5-yl)propanoic acid (0.16 g, 0.76 mmol), HATU (0.35 g, $0.92 \mathrm{mmol})$, HOAT (0.12 g, $0.92 \mathrm{mmol})$, DIPEA (0.24 g, $1.90 \mathrm{mmol}, 0.33$ $\mathrm{mL})$ and 2-(1,3-dioxolan-2-yl)aniline $(0.15 \mathrm{~g}, 0.92 \mathrm{mmol})$ in $4 \mathrm{~mL}$ of DMF. The product was deprotected in $8 \mathrm{~mL}$ of acetone with $\mathrm{TsOH} \cdot \mathrm{H}_{2} \mathrm{O}(0.03 \mathrm{~g}, 0.15 \mathrm{mmol})$. Purification by flash column chromatography gave $0.10 \mathrm{~g}(35 \%)$ of $2 \mathrm{e} .{ }^{1} \mathrm{H}$ NMR $\left(500 \mathrm{MHz}, \mathrm{CDCl}_{3}\right) \delta 11.24(\mathrm{~s}, 1 \mathrm{H}), 9.91(\mathrm{~d}, J$ $=0.7 \mathrm{~Hz}, 1 \mathrm{H}), 8.73(\mathrm{~d}, J=8.5 \mathrm{~Hz}, 1 \mathrm{H}), 7.97(\mathrm{~s}, 1 \mathrm{H}), 7.68(\mathrm{dd}, J=7.7,1.6 \mathrm{~Hz}, 1 \mathrm{H}), 7.66-7.59$ (m, 1H), $7.61(\mathrm{~s}, 1 \mathrm{H}), 7.25(\mathrm{td}, J=7.5,1.1 \mathrm{~Hz}, 1 \mathrm{H}), 6.99$ (s, 1H), $3.21(\mathrm{td}, J=7.4,1.1 \mathrm{~Hz}, 2 \mathrm{H})$, $2.88(\mathrm{dd}, J=8.0,6.9 \mathrm{~Hz}, 2 \mathrm{H}) .{ }^{13} \mathrm{C} \mathrm{NMR}\left(126 \mathrm{MHz}, \mathrm{CDCl}_{3}\right) \delta 195.82,170.27,151.91,151.66$, 151.63, 141.14, 140.75, 136.47, 136.25, 126.81, 124.86, 123.36, 121.74, 120.08, 35.90, 21.32 (acetone peaks observed in ${ }^{13} \mathrm{C}$ NMR). HRMS (ESI) calcd for $\mathrm{C}_{16} \mathrm{H}_{14} \mathrm{~N}_{3} \mathrm{O}_{4}\left(\mathrm{MH}^{+}\right) 312.0979$, found 312.0974 . 


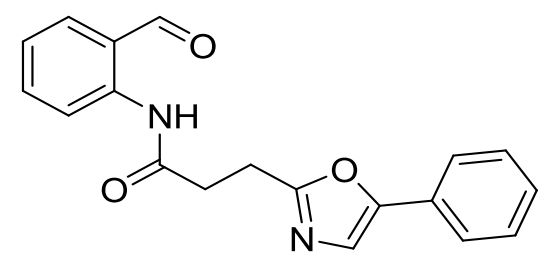

\section{N-(2-formylphenyl)-3-(5-phenyloxazol-2-yl)propanamide}

(9f): General procedure D was followed using3-(5-phenyl)oxazol-2-yl)propanoic acid (1.52 g, $7.00 \mathrm{mmol})$, HATU ( $3.19 \mathrm{~g}, 8.39 \mathrm{mmol})$, HOAT (1.14 g, $8.39 \mathrm{mmol})$, DIPEA (2.26 g, $17.47 \mathrm{mmol}$, $3.04 \mathrm{~mL})$ and 2-(1,3-dioxolan-2-yl)aniline $(1.38 \mathrm{~g}, 8.39 \mathrm{mmol})$ in $15 \mathrm{~mL}$ of DMF. The product was deprotected in $20 \mathrm{~mL}$ of acetone with $\mathrm{TsOH} \cdot \mathrm{H}_{2} \mathrm{O}(0.24 \mathrm{~g}, 1.40 \mathrm{mmol})$. Purification by flash column chromatography gave $1.66 \mathrm{~g}(74 \%)$ of 9 f. ${ }^{1} \mathrm{H}$ NMR $\left(500 \mathrm{MHz}, \mathrm{CDCl}_{3}\right) \delta 11.29(\mathrm{~s}, 1 \mathrm{H})$, $9.92(\mathrm{~d}, J=0.7 \mathrm{~Hz}, 1 \mathrm{H}), 8.75(\mathrm{~d}, J=8.4 \mathrm{~Hz}, 1 \mathrm{H}), 7.67$ (dd, $J=7.7,1.7 \mathrm{~Hz}, 1 \mathrm{H}), 7.64-7.57$ (m, $3 \mathrm{H}), 7.42-7.36(\mathrm{~m}, 2 \mathrm{H}), 7.33-7.27(\mathrm{~m}, 1 \mathrm{H}), 7.26-7.21(\mathrm{~m}, 2 \mathrm{H}), 3.29(\mathrm{dd}, J=8.1,6.8 \mathrm{~Hz}, 2 \mathrm{H})$, $3.05(\mathrm{dd}, J=8.1,6.9 \mathrm{~Hz}, 2 \mathrm{H}) .{ }^{13} \mathrm{C} \mathrm{NMR}\left(126 \mathrm{MHz}, \mathrm{CDCl}_{3}\right) \delta 195.62,170.62,162.85,151.42$, 140.92, 136.34, 136.15, 128.96, 128.33, 128.20, 124.19, 123.14, 122.00, 121.75, 120.12, 34.66, 23.71. HRMS (ESI) calcd for $\mathrm{C}_{19} \mathrm{H}_{16} \mathrm{~N}_{2} \mathrm{O}_{3} \mathrm{Li}\left(\mathrm{MLi}^{+}\right)$327.1316, found 327.1326.

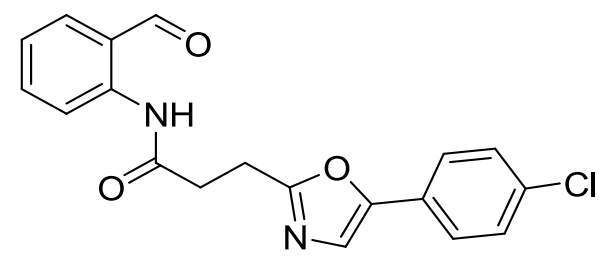

\section{3-(5-(4-chlorophenyl)oxazol-2-yl)-N-(2-formylphenyl)}

propanamide (9g): General procedure D was followed using3-(5-(4-chlorophenyl)oxazol-2yl)propanoic acid (0.35 g, $1.39 \mathrm{mmol})$, HATU (0.63 g, $1.67 \mathrm{mmol})$, HOAT (0.23 g, $1.67 \mathrm{mmol})$, DIPEA (0.45 g, $3.47 \mathrm{mmol}, 0.60 \mathrm{~mL})$ and 2-(1,3-dioxolan-2-yl)aniline $(0.27 \mathrm{~g}, 1.67 \mathrm{mmol})$ in 5 $\mathrm{mL}$ of DMF. The product was deprotected in $5 \mathrm{~mL}$ of acetone with $\mathrm{TsOH} \cdot \mathrm{H}_{2} \mathrm{O}(0.05 \mathrm{~g}, 0.10$ mmol). Purification by flash column chromatography gave $0.27 \mathrm{~g} \mathrm{(55 \% )} \mathrm{of} \mathrm{1g.}{ }^{1} \mathrm{H}$ NMR (500 $\left.\mathrm{MHz}, \mathrm{CDCl}_{3}\right) \delta 11.28(\mathrm{~s}, 1 \mathrm{H}), 9.91(\mathrm{~d}, J=0.7 \mathrm{~Hz}, 1 \mathrm{H}), 8.74(\mathrm{~d}, J=8.4 \mathrm{~Hz}, 1 \mathrm{H}), 7.67$ (dd, $J=7.6$, $1.6 \mathrm{~Hz}, 1 \mathrm{H}), 7.64-7.57(\mathrm{~m}, 1 \mathrm{H}), 7.52(\mathrm{~d}, J=8.7 \mathrm{~Hz}, 2 \mathrm{H}), 7.35(\mathrm{~d}, J=8.7 \mathrm{~Hz}, 2 \mathrm{H}), 7.23(\mathrm{td}, J=$ $7.5,1.0 \mathrm{~Hz}, 1 \mathrm{H}), 7.21(\mathrm{~s}, 1 \mathrm{H}), 3.27(\mathrm{dd}, J=7.9,6.9 \mathrm{~Hz}, 2 \mathrm{H}), 3.04(\mathrm{dd}, J=7.9,6.9 \mathrm{~Hz}, 2 \mathrm{H}) .{ }^{13} \mathrm{C}$ NMR $\left(126 \mathrm{MHz}, \mathrm{CDCl}_{3}\right) \delta 195.63,170.52,163.11,150.41,140.86,136.34,136.15,134.03$, 129.20, 126.67, 125.40, 123.16, 122.39, 121.72, 120.07, 34.55, 23.65. HRMS (ESI) calcd for $\mathrm{C}_{19} \mathrm{H}_{15} \mathrm{~N}_{2} \mathrm{O}_{3} \mathrm{ClLi}\left(\mathrm{MLi}^{+}\right)$361.0926, found 361.0937. 


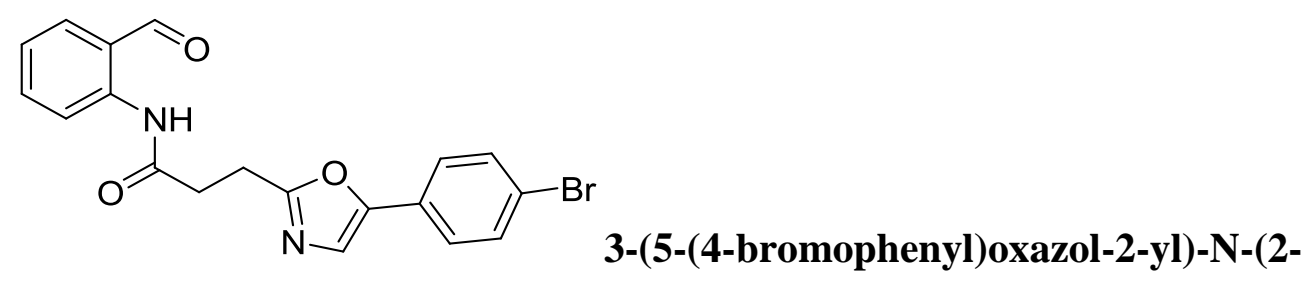

formylphenyl)propanamide (9h): General procedure D was followed using3-(5-(4bromophenyl)oxazol-2-yl)propanoic acid (0.52 g, $1.75 \mathrm{mmol}$ ), HATU (0.80 g, $2.10 \mathrm{mmol}$ ), HOAT (0.28 g, $2.10 \mathrm{mmol})$, DIPEA (0.56 g, $4.37 \mathrm{mmol}, 0.76 \mathrm{~mL})$ and 2-(1,3-dioxolan-2-yl)aniline (0.35 $\mathrm{g}, 2.10 \mathrm{mmol}$ ) in $6 \mathrm{~mL}$ of DMF. The product was deprotected in $10 \mathrm{~mL}$ of acetone with $\mathrm{TsOH} \cdot \mathrm{H}_{2} \mathrm{O}$ $(0.07 \mathrm{~g}, 0.35 \mathrm{mmol})$ stirring overnight at room temperature. Purification by flash column chromatography gave $0.62 \mathrm{~g}(89 \%)$ of $9 \mathrm{~h} .{ }^{1} \mathrm{H}$ NMR $\left(500 \mathrm{MHz}, \mathrm{CDCl}_{3}\right) \delta 11.28(\mathrm{~s}, 1 \mathrm{H}), 9.91(\mathrm{~d}$, $J=0.8 \mathrm{~Hz}, 1 \mathrm{H}), 8.74(\mathrm{~d}, J=8.5 \mathrm{~Hz}, 1 \mathrm{H}), 7.67(\mathrm{dd}, J=7.7,1.7 \mathrm{~Hz}, 1 \mathrm{H}), 7.61$ (ddd, $J=8.8,7.3$, $1.7 \mathrm{~Hz}, 1 \mathrm{H}), 7.50(\mathrm{~d}, J=8.7 \mathrm{~Hz}, 2 \mathrm{H}), 7.45(\mathrm{~d}, J=8.7 \mathrm{~Hz}, 2 \mathrm{H}), 7.23(\mathrm{td}, J=7.5,1.0 \mathrm{~Hz}, 1 \mathrm{H}), 7.22$ $(\mathrm{s}, 1 \mathrm{H}), 3.27(\mathrm{dd}, J=7.9,6.8 \mathrm{~Hz}, 2 \mathrm{H}), 3.04(\mathrm{dd}, J=7.8,6.9 \mathrm{~Hz}, 2 \mathrm{H}) .{ }^{13} \mathrm{C} \mathrm{NMR}\left(126 \mathrm{MHz}, \mathrm{CDCl}_{3}\right)$ $\delta 195.64,170.51,163.17,150.44,140.86,136.34,136.16,132.14,127.10,125.64,123.17,122.51$, 122.16, 121.72, 120.08, 34.54, 23.66. HRMS (ESI) calcd for $\mathrm{C}_{19} \mathrm{H}_{16} \mathrm{BrN}_{2} \mathrm{O}_{3}\left(\mathrm{MH}^{+}\right)$399.0339, found 399.0341 . 


\section{PHOTOCHEMISTRY}

Irradiation of photoprecursors 2a-e.<smiles>[R]c1ncc(CCC(=O)Nc2ccccc2C=O)o1</smiles>

2a-e

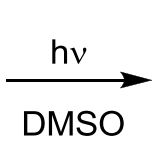

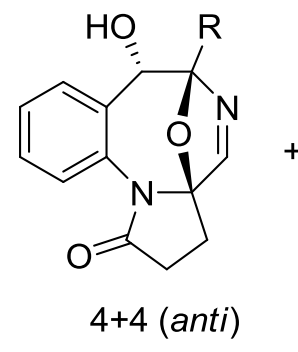

3a-e

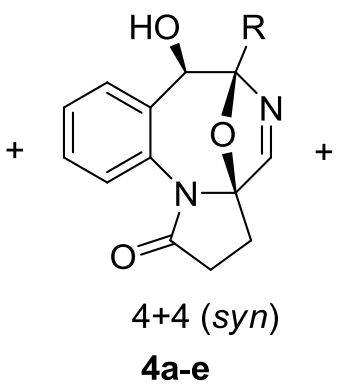

4a-e

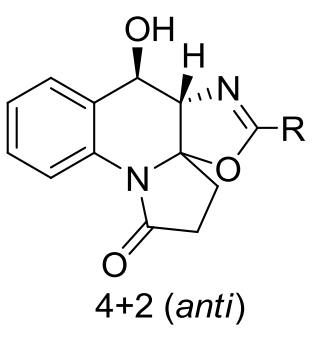

5a-e

General Procedure E for irradiations: Approximately 0.03M solution of a photoprecursor 2 in DMSO was degassed by bubbling with nitrogen for $30 \mathrm{~min}$ and irradiated with UV LED-based illuminator with five $250 \mathrm{~mW} @ 365 \mathrm{~nm}$ Nichia LEDs for $3 \mathrm{~h}$. The solvent was removed under vacuum and the products were purified by flash column chromatography.

Irradiation of 2a. General Procedure E was followed on $0.19 \mathrm{~g}(0.59 \mathrm{mmol})$ of photoprecursor $\mathbf{2 a}$ in $20 \mathrm{~mL}$ of DMSO, 3h irradiation. The products were purified by flash column chromatography affording:<smiles>O=C1CC[C@]23C=N[C@](c4ccccc4)(O2)[C@H](O)c2ccccc2N13</smiles>

anti-8-hydroxy-9-phenyl-6,7-benzo-4,12-dioxa-5,10-diaza-

tricyclo[7.2.1.0 ${ }^{1,5}$ ]dodeca-6,10-diene (3a): $0.07 \mathrm{~g}(34 \%) .{ }^{1} \mathrm{H}$ NMR (500 MHz, CDCl3) $\delta 7.86$ $(\mathrm{dt}, J=8.0,1.4 \mathrm{~Hz}, 1 \mathrm{H}), 7.81-7.76(\mathrm{~m}, 2 \mathrm{H}), 7.59(\mathrm{dd}, J=8.0,1.4 \mathrm{~Hz}, 1 \mathrm{H}), 7.53-7.43(\mathrm{~m}, 4 \mathrm{H})$, 7.35 (dddd, $J=8.0,7.3,1.6,0.8 \mathrm{~Hz}, 1 \mathrm{H}), 7.30-7.26$ (m, 1H), 5.04 (dt, $J=6.3,1.0 \mathrm{~Hz}, 1 \mathrm{H}), 2.96$ (ddd, $J=17.3,10.1,9.2 \mathrm{~Hz}, 1 \mathrm{H}), 2.71$ (ddd, $J=17.3,10.0,1.4 \mathrm{~Hz}, 1 \mathrm{H}), 2.61$ (dt, $J=13.9,10.0$ $\mathrm{Hz}, 1 \mathrm{H}), 2.38(\mathrm{ddd}, J=13.9,9.2,1.3 \mathrm{~Hz}, 1 \mathrm{H}), 2.00(\mathrm{~d}, J=6.2 \mathrm{~Hz}, 1 \mathrm{H}) .{ }^{13} \mathrm{C} \mathrm{NMR}(126 \mathrm{MHz}$, 
$\left.\mathrm{CDCl}_{3}\right) \delta 172.55,161.04,138.15,132.21,131.46,129.36,128.88,128.31,128.03,127.14,126.81$, 126.53, 110.70, 102.54, 78.74, 30.05, 26.89. HRMS (ESI) calcd for $\mathrm{C}_{19} \mathrm{H}_{17} \mathrm{~N}_{2} \mathrm{O}_{3}\left(\mathrm{MH}^{+}\right) 321.1233$, found 321.1239 .

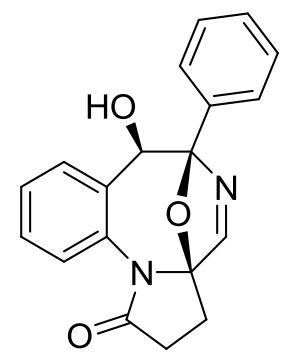

syn-8-hydroxy-9-phenyl-6,7-benzo-4,12-dioxa-5,10-diaza-tricyclo[7.2.1.0 $\left.{ }^{1,5}\right]$ dodeca-6,10-diene (4a): $0.06 \mathrm{~g}(32 \%) .{ }^{1} \mathrm{H}$ NMR (500 MHz, $\left.\mathrm{CDCl}_{3}\right) \delta 7.83-7.78(\mathrm{~m}, 2 \mathrm{H}), 7.66$ $(\mathrm{dd}, J=8.2,1.3 \mathrm{~Hz}, 1 \mathrm{H}), 7.49-7.43(\mathrm{~m}, 2 \mathrm{H}), 7.43-7.35(\mathrm{~m}, 2 \mathrm{H}), 7.32(\mathrm{dd}, J=7.7,1.7 \mathrm{~Hz}, 1 \mathrm{H})$, 7.29 (s, 1H), $7.26-7.21(\mathrm{~m}, 1 \mathrm{H}), 5.02$ (d, $J=10.2 \mathrm{~Hz}, 1 \mathrm{H}), 3.03$ (dt, $J=16.4,9.4 \mathrm{~Hz}, 1 \mathrm{H}), 2.80$ - $2.62(\mathrm{~m}, 2 \mathrm{H}), 2.56-2.49(\mathrm{~m}, 1 \mathrm{H}), 2.47(\mathrm{dd}, J=10.1,1.1 \mathrm{~Hz}, 1 \mathrm{H}) .{ }^{13} \mathrm{C} \mathrm{NMR}\left(126 \mathrm{MHz}, \mathrm{CDCl}_{3}\right)$ $\delta 172.72,159.04,137.69,133.32,132.11,131.66,129.12,128.87,128.47,127.22,126.80,126.65$, 110.30, 103.91, 83.31, 29.94, 26.95. HRMS (ESI) calcd for $\mathrm{C}_{19} \mathrm{H}_{17} \mathrm{~N}_{2} \mathrm{O}_{3}\left(\mathrm{MH}^{+}\right)$321.1233, found 321.1229.<smiles>O=C(N[C@H]1[C@H](O)c2ccccc2N2C(=O)CC[C@]12O)c1ccccc1</smiles>

\section{N-((3aS,4S,5R)-3a,5-dihydroxy-1-oxo-1,2,3,3a,4,5-hexahydropyrrolo}

[1,2-a]quinolin-4-yl)benzamide (5a): General Procedure E followed on $0.19 \mathrm{~g}(0.59 \mathrm{mmol})$ of aldehyde (1a) in $20 \mathrm{~mL}$ of DMSO, and irradiated for $3 \mathrm{~h}$. The product was purified by flash column chromatography which afforded $0.03 \mathrm{~g}(14 \%)$.of quinolinol 3a. ${ }^{1} \mathrm{H}$ NMR (500 MHz, DMSO- $\left.d_{6}\right)$ $\delta 8.47(\mathrm{dd}, J=8.3,1.2 \mathrm{~Hz}, 1 \mathrm{H}), 8.32(\mathrm{~d}, J=9.0 \mathrm{~Hz}, 1 \mathrm{H}), 8.05-7.92(\mathrm{~m}, 2 \mathrm{H}), 7.60-7.53(\mathrm{~m}, 2 \mathrm{H})$, $7.53-7.46(\mathrm{~m}, 2 \mathrm{H}), 7.32-7.23(\mathrm{~m}, 1 \mathrm{H}), 7.15(\mathrm{td}, J=7.5,1.3 \mathrm{~Hz}, 1 \mathrm{H}), 6.46(\mathrm{~d}, J=1.4 \mathrm{~Hz}, 1 \mathrm{H})$, $5.51(\mathrm{~d}, J=8.1 \mathrm{~Hz}, 1 \mathrm{H}), 4.89(\mathrm{dd}, J=10.3,8.2 \mathrm{~Hz}, 1 \mathrm{H}), 4.30(\mathrm{ddd}, J=10.3,8.9,1.4 \mathrm{~Hz}, 1 \mathrm{H}), 2.74$ $(\mathrm{dt}, J=16.5,9.4 \mathrm{~Hz}, 1 \mathrm{H}), 2.44-2.26(\mathrm{~m}, 2 \mathrm{H}), 1.93(\mathrm{ddd}, J=13.4,9.4,2.2 \mathrm{~Hz}, 1 \mathrm{H}) .{ }^{13} \mathrm{C}$ NMR (126 MHz, DMSO) $\delta 172.91,167.30,134.47,133.64,131.24,129.87,128.14,127.79,127.61$, 
127.41, 123.51, 117.95, 90.84, 66.67, 57.89, 30.00, 29.85. HRMS (ESI) calcd for $\mathrm{C}_{19} \mathrm{H}_{19} \mathrm{~N}_{2} \mathrm{O}_{4}$ $\left(\mathrm{MH}^{+}\right)$339.1339, found 339.1344.

Irradiation of $\mathbf{2 b}$ : General Procedure E followed on $0.20 \mathrm{~g}(0.57 \mathrm{mmol})$ of photoprecursor $\mathbf{2 b}$ in $20 \mathrm{~mL}$ of DMSO, 3h irradiation. The product was purified by flash column chromatography which afforded:

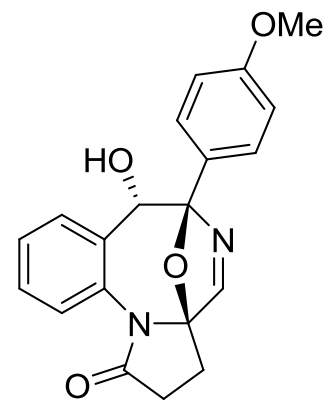

anti-8-hydroxy-9-(4-methoxyphenyl)-6,7-benzo-4,12-dioxa-5,10-diazatricyclo[7.2.1.01,5]dodeca-6,10-diene (3b): $0.05 \mathrm{~g}(27 \%) .{ }^{1} \mathrm{H}$ NMR $\left(500 \mathrm{MHz}, \mathrm{CDCl}_{3}\right) \delta 7.86(\mathrm{dt}$, $J=8.1,1.4 \mathrm{~Hz}, 1 \mathrm{H}), 7.69(\mathrm{~d}, J=8.8,2 \mathrm{H}), 7.58(\mathrm{dd}, J=8.0,1.4 \mathrm{~Hz}, 1 \mathrm{H}), 7.48(\mathrm{~s}, 1 \mathrm{H}), 7.36-7.31$ $(\mathrm{m}, 1 \mathrm{H}), 7.29-7.24(\mathrm{~m}, 1 \mathrm{H}), 7.03-6.98(\mathrm{~d}, J=8.8,2 \mathrm{H}), 4.99(\mathrm{~d}, J=5.8 \mathrm{~Hz}, 1 \mathrm{H}), 3.85(\mathrm{~s}, 3 \mathrm{H})$, $2.99-2.91(\mathrm{~m}, 1 \mathrm{H}), 2.70(\mathrm{ddd}, J=17.3,9.9,1.4 \mathrm{~Hz}, 1 \mathrm{H}), 2.60(\mathrm{dt}, J=13.9,10.0 \mathrm{~Hz}, 1 \mathrm{H}), 2.36$ $(\mathrm{ddd}, J=13.9,9.2,1.3 \mathrm{~Hz}, 1 \mathrm{H}), 2.07(\mathrm{~d}, J=5.8 \mathrm{~Hz}, 1 \mathrm{H}) .{ }^{13} \mathrm{C} \mathrm{NMR}\left(126 \mathrm{MHz}, \mathrm{CDCl}_{3}\right) \delta 172.56$, $160.87,160.37,132.24,131.47,130.12$, 128.43, 128.32, 127.96, 126.77, 126.48, 114.28, 110.58, 102.56, 78.69, 55.54, 30.05, 26.92. HRMS (ESI) calcd for $\mathrm{C}_{20} \mathrm{H}_{19} \mathrm{~N}_{2} \mathrm{O}_{4}\left(\mathrm{MH}^{+}\right) 351.1339$, found 351.1338 .

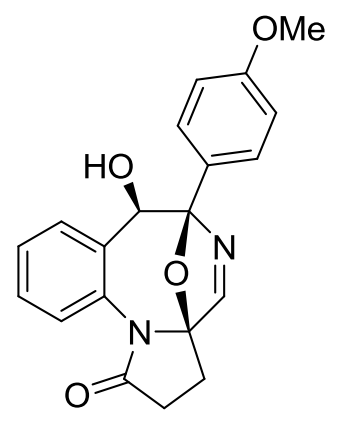

syn-8-hydroxy-9-(4-methoxyphenyl)-6,7-benzo-4,12-dioxa-5,10-diazatricyclo[7.2.1.0 ${ }^{1,5}$ ]dodeca-6,10-diene (4b): $0.07 \mathrm{~g}(34 \%) .{ }^{1} \mathrm{H}$ NMR $\left(500 \mathrm{MHz}, \mathrm{CDCl}_{3}\right) \delta 7.75-$ $7.68(\mathrm{~m}, 2 \mathrm{H}), 7.65(\mathrm{dd}, J=8.2,1.3 \mathrm{~Hz}, 1 \mathrm{H}), 7.37(\mathrm{ddd}, J=8.2,7.3,1.7 \mathrm{~Hz}, 1 \mathrm{H}), 7.31$ (dd, $J=7.7$, $1.7 \mathrm{~Hz}, 1 \mathrm{H}), 7.27(\mathrm{~s}, 1 \mathrm{H}), 7.23(\mathrm{~d}, J=1.4 \mathrm{~Hz}, 1 \mathrm{H}), 7.00-6.96(\mathrm{~m}, 2 \mathrm{H}), 4.99$ (d, $J=9.4 \mathrm{~Hz}, 1 \mathrm{H})$, 
$3.84(\mathrm{~s}, 3 \mathrm{H}), 3.03(\mathrm{dt}, J=16.5,9.4 \mathrm{~Hz}, 1 \mathrm{H}), 2.80-2.71(\mathrm{~m}, 1 \mathrm{H}), 2.71-2.64(\mathrm{~m}, 1 \mathrm{H}), 2.56-2.48$ $(\mathrm{m}, 1 \mathrm{H}), 2.44(\mathrm{~d}, J=10.1 \mathrm{~Hz}, 1 \mathrm{H}) .{ }^{13} \mathrm{C} \mathrm{NMR}\left(126 \mathrm{MHz}, \mathrm{CDCl}_{3}\right) \delta 172.76,160.02,158.86,133.30$, 132.12, 131.77, 129.89, 129.04, 127.94, 127.17, 126.78, 113.88, 110.16, 103.90, 83.23, 55.52, 29.92, 26.97. HRMS (ESI) calcd for $\mathrm{C}_{20} \mathrm{H}_{18} \mathrm{~N}_{2} \mathrm{O}_{4} \mathrm{Li}\left(\mathrm{MLi}^{+}\right)$357.1421, found 357.1427.<smiles>COc1ccc(C(=O)NC2CC3(C)CCC(=O)N3c3ccccc3[C@@H]2O)cc1</smiles>

N-((3aS,4S,5R)-3a,5-dihydroxy-1-oxo-1,2,3,3a,4,5hexahydropyrrolo[1,2-a]quinolin-4-yl)-4-methoxybenzamide (5b): $0.03 \mathrm{~g}(15 \%) .{ }^{1} \mathrm{H}$ NMR $\left(500 \mathrm{MHz}, \mathrm{DMSO}-d_{6}\right) \delta 8.44(\mathrm{~d}, J=8.2 \mathrm{~Hz}, 1 \mathrm{H}), 8.15(\mathrm{~d}, J=9.0 \mathrm{~Hz}, 1 \mathrm{H}), 7.96(\mathrm{~d}, J=8.8 \mathrm{~Hz}$, 2H), $7.57(\mathrm{~d}, J=7.7 \mathrm{~Hz}, 1 \mathrm{H}), 7.26(\mathrm{t}, J=7.9 \mathrm{~Hz}, 1 \mathrm{H}), 7.14(\mathrm{t}, J=7.8 \mathrm{~Hz}, 1 \mathrm{H}), 7.02$ (d, $J=8.8 \mathrm{~Hz}$, 2H), $6.52(\mathrm{~d}, J=1.4 \mathrm{~Hz}, 1 \mathrm{H}), 5.54(\mathrm{~d}, J=8.1 \mathrm{~Hz}, 1 \mathrm{H}), 4.88(\mathrm{t}, J=9.2 \mathrm{~Hz}, 1 \mathrm{H}), 4.27(\mathrm{t}, J=9.6 \mathrm{~Hz}$, 1H), $3.82(\mathrm{~s}, 3 \mathrm{H}), 2.73(\mathrm{dt}, J=16.4,9.4 \mathrm{~Hz}, 1 \mathrm{H}), 2.43-2.34(\mathrm{~m}, 1 \mathrm{H}), 2.34-2.25(\mathrm{~m}, 1 \mathrm{H}), 1.90$ (ddd, $J=12.9,9.6,2.1 \mathrm{~Hz}, 1 \mathrm{H}) .{ }^{13} \mathrm{C}$ NMR $\left(126 \mathrm{MHz}, \mathrm{DMSO}-\mathrm{d}_{6}\right) \delta 173.10,166.95,161.78,133.72$, 130.02, 129.61, 127.93, 127.57, 126.66, 123.71, 118.15, 113.51, 91.04, 66.85, 57.90, 55.50, 30.07, 29.97. HRMS (ESI) calcd for $\mathrm{C}_{20} \mathrm{H}_{20} \mathrm{~N}_{2} \mathrm{O}_{5} \mathrm{Li}\left(\mathrm{MLi}^{+}\right)$375.1527, found 375.1529.

Irradiation of 2c: General Procedure E followed on $0.20 \mathrm{~g}(0.64 \mathrm{mmol})$ of photoprecursor $2 c$ in $25 \mathrm{~mL}$ of DMSO, 3h irradiation. The product was purified by flash column chromatography which afforded:

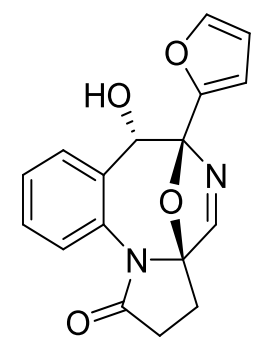

anti-8-hydroxy-9-(furan-2-yl)-6,7-benzo-4,12-dioxa-5,10-diaza-tricyclo [7.2.1.01,5] dodeca-6,10-diene (3c): $0.08 \mathrm{~g}(39 \%)$. ${ }^{1} \mathrm{H}$ NMR $\left(500 \mathrm{MHz}, \mathrm{CDCl}_{3}\right) \delta 7.86$ (dt, $J=8.0$, $1.4 \mathrm{~Hz}, 1 \mathrm{H}), 7.59-7.54$ (m, 2H), 7.54 (s, 1H), 7.34 (dddd, $J=8.0,7.2,1.7,0.8 \mathrm{~Hz}, 1 \mathrm{H}), 7.27$ 
(ddd, $J=8.7,7.3,1.5 \mathrm{~Hz}, 1 \mathrm{H}), 6.63(\mathrm{dd}, J=3.3,0.9 \mathrm{~Hz}, 1 \mathrm{H}), 6.47(\mathrm{dd}, J=3.3,1.8 \mathrm{~Hz}, 1 \mathrm{H}), 5.29$ $(\mathrm{dt}, J=8.4,1.0 \mathrm{~Hz}, 1 \mathrm{H}), 2.93(\mathrm{ddd}, J=15.9,9.5,8.9 \mathrm{~Hz}, 1 \mathrm{H}), 2.73-2.58(\mathrm{~m}, 2 \mathrm{H}), 2.48(\mathrm{~d}, J=$ $8.5 \mathrm{~Hz}, 1 \mathrm{H}), 2.44-2.37(\mathrm{~m}, 1 \mathrm{H}) .{ }^{13} \mathrm{C} \mathrm{NMR}\left(126 \mathrm{MHz}, \mathrm{CDCl}_{3}\right) \delta 172.43,162.75,150.44,143.71$, $131.85,131.15,128.43,128.11,126.89,126.59$, 110.76, 110.18, 106.98, 103.15, 76.84, 29.86, 26.74. HRMS (ESI) calcd for $\mathrm{C}_{17} \mathrm{H}_{15} \mathrm{~N}_{2} \mathrm{O}_{4}\left(\mathrm{MH}^{+}\right) 311.1026$, found 311.1031. (X-ray - see cif)

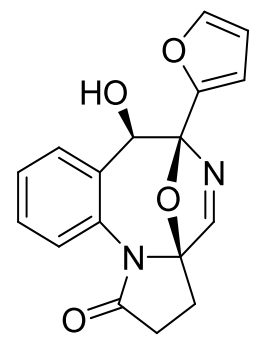

syn-8-hydroxy-9-(furan-2-yl)-6,7-benzo-4,12-dioxa-5,10-diazatricyclo[7.2.1.0 ${ }^{1,5}$ ]dodeca-6,10-diene (4c): $0.06 \mathrm{~g}(31 \%) .{ }^{1} \mathrm{H} \mathrm{NMR}\left(500 \mathrm{MHz}, \mathrm{CDCl}_{3}\right) \delta 7.64-$ $7.59(\mathrm{~m}, 1 \mathrm{H}), 7.53(\mathrm{dd}, J=1.8,0.9 \mathrm{~Hz}, 1 \mathrm{H}), 7.38-7.35(\mathrm{~m}, 2 \mathrm{H}), 7.33(\mathrm{~s}, 1 \mathrm{H}), 7.24(\mathrm{dd}, J=7.4$, $1.3 \mathrm{~Hz}, 1 \mathrm{H}), 6.71(\mathrm{dd}, J=3.3,0.9 \mathrm{~Hz}, 1 \mathrm{H}), 6.46(\mathrm{dd}, J=3.4,1.8 \mathrm{~Hz}, 1 \mathrm{H}), 5.32$ (d, $J=10.3 \mathrm{~Hz}$, 1H), $3.03-2.87(\mathrm{~m}, 1 \mathrm{H}), 2.74(\mathrm{~d}, J=10.3 \mathrm{~Hz}, 1 \mathrm{H}) 2.73-2.66(\mathrm{~m}, 2 \mathrm{H}), 2.55(\mathrm{dd}, J=12.2,9.3$ $\mathrm{Hz}, 1 \mathrm{H}) .{ }^{13} \mathrm{C} \mathrm{NMR}\left(126 \mathrm{MHz}, \mathrm{CDCl}_{3}\right) \delta 172.76,160.15,149.82,143.48,133.39,131.97,131.26$, 129.24, 127.34, 126.89, 110.76, 109.66, 106.45, 103.89, 80.30, 29.77, 26.84. HRMS (ESI) calcd for $\mathrm{C}_{17} \mathrm{H}_{15} \mathrm{~N}_{2} \mathrm{O}_{4}\left(\mathrm{MH}^{+}\right)$311.1026, found 311.1032.

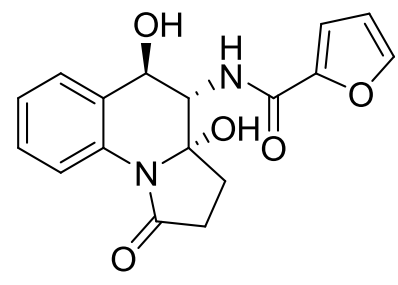

\section{N-((3aS,4S,5R)-3a,5-dihydroxy-1-oxo-1,2,3,3a,4,5-hexahydropyrrolo}

[1,2-a]quinolin-4-yl)furan-2-carboxamide (5c): $0.04 \mathrm{~g}(17 \%) .{ }^{1} \mathrm{H} \mathrm{NMR}\left(500 \mathrm{MHz},\left(\mathrm{CD}_{3}\right)_{2} \mathrm{SO}\right)$ $\delta 8.45(\mathrm{dd}, J=8.4,1.2 \mathrm{~Hz}, 1 \mathrm{H}), 8.02(\mathrm{~d}, J=9.3 \mathrm{~Hz}, 1 \mathrm{H}), 7.88(\mathrm{dd}, J=1.8,0.8 \mathrm{~Hz}, 1 \mathrm{H}), 7.55(\mathrm{dt}$, $J=7.6,1.3 \mathrm{~Hz}, 1 \mathrm{H}), 7.31-7.24(\mathrm{~m}, 2 \mathrm{H}), 7.14(\mathrm{td}, J=7.5,1.3 \mathrm{~Hz}, 1 \mathrm{H}), 6.66(\mathrm{dd}, J=3.5,1.8 \mathrm{~Hz}$, 1H), 6.60 (d, $J=1.2 \mathrm{~Hz}, 1 \mathrm{H}), 5.61(\mathrm{~d}, J=8.1 \mathrm{~Hz}, 1 \mathrm{H}), 4.88-4.75(\mathrm{~m}, 1 \mathrm{H}), 4.20$ (ddd, $J=10.3$, 9.2, 1.1 Hz, 1H), $2.74(\mathrm{dt}, J=17.0,9.6 \mathrm{~Hz}, 1 \mathrm{H}), 2.38$ (ddd, $J=17.0,10.0,2.4 \mathrm{~Hz}, 1 \mathrm{H}), 2.24(\mathrm{dt}, J$ $=13.2,9.8 \mathrm{~Hz}, 1 \mathrm{H}), 1.92(\mathrm{ddd}, J=13.3,9.5,2.4 \mathrm{~Hz}, 1 \mathrm{H}) .{ }^{13} \mathrm{C} \mathrm{NMR}\left(126 \mathrm{MHz},\left(\mathrm{CD}_{3}\right)_{2} \mathrm{SO}\right) \delta$ 173.08, 158.63, 147.59, 145.33, 133.66, 129.74, 127.88, 127.64, 123.74, 118.13, 114.16, 112.10, 90.78, 66.92, 57.35, 30.16, 29.93. HRMS (ESI) calcd for $\mathrm{C}_{17} \mathrm{H}_{16} \mathrm{~N}_{2} \mathrm{O}_{5} \mathrm{Li}\left(\mathrm{MLi}^{+}\right)$335.1214, found 335.1215 . 
Irradiation of 2d: General Procedure E followed on $0.18 \mathrm{~g}(0.55 \mathrm{mmol})$ of photoprecursor $2 \mathbf{d}$ in $18 \mathrm{~mL}$ of DMSO, 3h irradiation. The product was purified by flash column chromatography which afforded:<smiles>O=C1CC[C@]23C=N[C@](c4cccs4)(O2)[C@H](O)c2ccccc2N13</smiles>

anti-8-hydroxy-9-(thiophen-2-yl)-6,7-benzo-4,12-dioxa-5,10-diazatricyclo [7.2.1.0 ${ }^{1,5}$ ]dodeca-6,10-diene (3d): $0.04 \mathrm{~g}(23 \%)$. ${ }^{1} \mathrm{H} \mathrm{NMR}\left(500 \mathrm{MHz}, \mathrm{CDCl}_{3}\right) \delta 7.89$ (dt, $J=8.1$, $1.4 \mathrm{~Hz}, 1 \mathrm{H}), 7.58(\mathrm{dd}, J=8.0,1.4 \mathrm{~Hz}, 1 \mathrm{H}), 7.50(\mathrm{~s}, 1 \mathrm{H}), 7.46-7.40(\mathrm{~m}, 2 \mathrm{H}), 7.38-7.32(\mathrm{~m}, 1 \mathrm{H})$, $7.28(\mathrm{dd}, J=8.0,1.4 \mathrm{~Hz}, 1 \mathrm{H}), 7.15(\mathrm{dd}, J=5.1,3.6 \mathrm{~Hz}, 1 \mathrm{H}), 5.05(\mathrm{~d}, J=6.4 \mathrm{~Hz}, 1 \mathrm{H}), 2.95(\mathrm{dt}, J$ $=17.0,9.5 \mathrm{~Hz}, 1 \mathrm{H}), 2.76-2.57(\mathrm{~m}, 2 \mathrm{H}), 2.47-2.36(\mathrm{~m}, 1 \mathrm{H}), 2.28(\mathrm{dd}, J=6.5,1.4 \mathrm{~Hz}, 1 \mathrm{H}) .{ }^{13} \mathrm{C}$ NMR (126 MHz, $\left.\mathrm{CDCl}_{3}\right) \delta 172.44,161.53,140.32,131.71,131.35,128.52,128.14,127.50$, 126.96, 126.86, 126.65, 126.54, 109.14, 103.21, 78.74, 29.93, 26.86. HRMS (ESI) calcd for $\mathrm{C}_{17} \mathrm{H}_{14} \mathrm{~N}_{2} \mathrm{O}_{3} \mathrm{SLi}\left(\mathrm{MLi}^{+}\right)$333.0880, found 333.0881.

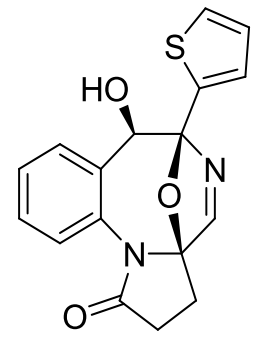

syn-8-hydroxy-9-(thiophen-2-yl)-6,7-benzo-4,12-dioxa-5,10-diazatricyclo[7.2.1.01,5]dodeca-6,10-diene (4d): $0.08 \mathrm{~g}(43 \%) .{ }^{1} \mathrm{H}$ NMR (500 MHz, $\left.\mathrm{CDCl}_{3}\right) \delta 7.63(\mathrm{~d}$, $J=8.2 \mathrm{~Hz}, 1 \mathrm{H}), 7.45(\mathrm{dt}, J=3.5,0.9 \mathrm{~Hz}, 1 \mathrm{H}), 7.41-7.35(\mathrm{~m}, 2 \mathrm{H}), 7.34-7.30(\mathrm{~m}, 2 \mathrm{H}), 7.23(\mathrm{~d}$, $J=7.6 \mathrm{~Hz}, 1 \mathrm{H}), 7.12-7.06(\mathrm{~m}, 1 \mathrm{H}), 5.11(\mathrm{~d}, J=10.1 \mathrm{~Hz}, 1 \mathrm{H}), 3.06-2.86(\mathrm{~m}, 1 \mathrm{H}), 2.72(\mathrm{dt}, J=$ 14.8, $9.6 \mathrm{~Hz}, 3 \mathrm{H}), 2.53$ (s, $1 \mathrm{H}) .{ }^{13} \mathrm{C} \mathrm{NMR}\left(126 \mathrm{MHz}, \mathrm{CDCl}_{3}\right) \delta 172.69,159.79,140.20,133.31$, $131.97,131.43$, 129.23, 127.29, 127.16, 126.84, 126.48, 126.39, 108.57, 104.37, 82.76, 29.82, 26.91. HRMS (ESI) calcd for $\mathrm{C}_{17} \mathrm{H}_{14} \mathrm{~N}_{2} \mathrm{O}_{3} \mathrm{SLi}\left(\mathrm{MLi}^{+}\right)$333.0880, found 333.0880. 
<smiles>O=C(NC1CCC(=O)N2c3ccccc3[C@@H](O)C12)c1cccs1</smiles>

N-((3aS,4S,5R)-3a,5-dihydroxy-1-oxo-1,2,3,3a,4,5-hexahydropyrrolo [1,2-a]quinolin-4-yl)thiophene-2-carboxamide (5d): $0.03 \mathrm{~g}(16 \%) .{ }^{1} \mathrm{H}$ NMR $(500 \mathrm{MHz}$, $\left.\mathrm{CD}_{3} \mathrm{OD}\right) \delta 8.48(\mathrm{dd}, J=8.4,1.3 \mathrm{~Hz}, 1 \mathrm{H}), 7.90(\mathrm{dd}, J=3.8,1.2 \mathrm{~Hz}, 1 \mathrm{H}), 7.70(\mathrm{dd}, J=5.0,1.1 \mathrm{~Hz}$, 1H), $7.68-7.61(\mathrm{~m}, 1 \mathrm{H}), 7.30$ (dddd, $J=8.2,7.3,1.6,0.8 \mathrm{~Hz}, 1 \mathrm{H}), 7.20$ (td, $J=7.5,1.3 \mathrm{~Hz}, 1 \mathrm{H})$, $7.17(\mathrm{dd}, J=5.0,3.8 \mathrm{~Hz}, 1 \mathrm{H}), 5.00$ (d, $J=10.1 \mathrm{~Hz}, 1 \mathrm{H}), 4.42(\mathrm{~d}, J=10.2 \mathrm{~Hz}, 1 \mathrm{H}), 2.90-2.79$ (m, $1 \mathrm{H}), 2.57-2.40(\mathrm{~m}, 2 \mathrm{H}), 2.07(\mathrm{ddd}, J=13.5,9.7,2.4 \mathrm{~Hz}, 1 \mathrm{H}) .{ }^{13} \mathrm{C} \mathrm{NMR}\left(126 \mathrm{MHz}, \mathrm{CD}_{3} \mathrm{OD}\right) \delta$ 174.04, 163.87, 138.63, 130.68, 129.10, 128.70, 127.62, 127.43, 127.35, 124.13, 118.95, 99.98, 91.38, 67.93, 58.37, 29.92, 29.67. HRMS (ESI) calcd for $\mathrm{C}_{17} \mathrm{H}_{16} \mathrm{~N}_{2} \mathrm{O}_{4} \mathrm{SLi}\left(\mathrm{MLi}^{+}\right)$351.0985, found 351.0986 .

Irradiation of 2e: General Procedure E followed on $0.09 \mathrm{~g}(0.28 \mathrm{mmol})$ of photoprecursor $2 \mathrm{e}$ in $9 \mathrm{~mL}$ of DMSO, $3 \mathrm{~h}$ irradiation. The product was purified by flash column chromatography which afforded:

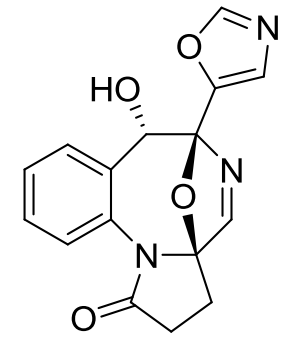

anti-8-hydroxy-9-(oxazol-5-yl)-6,7-benzo-4,12-dioxa-5,10-diazatricyclo [7.2.1.0 ${ }^{1,5}$ ]dodeca-6,10-diene (3e): $0.056 \mathrm{~g}(62 \%) .{ }^{1} \mathrm{H} \mathrm{NMR}\left(500 \mathrm{MHz}, \mathrm{CDCl}_{3}\right) \delta 7.99$ (d, $J=0.4$ $\mathrm{Hz}, 1 \mathrm{H}), 7.84$ (dt, $J=8.1,1.4 \mathrm{~Hz}, 1 \mathrm{H}), 7.59$ (s, 1H), 7.58 (d, $J=8.1,1.4 \mathrm{~Hz}, 1 \mathrm{H}), 7.37$ (dddd, $J=$ 8.1, 7.3, 1.7, $0.8 \mathrm{~Hz}, 1 \mathrm{H}), 7.33$ (s, 1H), 7.30 (ddd, $J=8.7,7.3,1.5 \mathrm{~Hz}, 1 \mathrm{H}), 5.24$ (dt, $J=10.6,0.9$ $\mathrm{Hz}, 1 \mathrm{H}), 2.98-2.87(\mathrm{~m}, 1 \mathrm{H}), 2.77-2.62(\mathrm{~m}, 2 \mathrm{H}), 2.52$ (dt, $J=10.6,1.4 \mathrm{~Hz}, 1 \mathrm{H}), 2.45-2.37$ (m, 1H). ${ }^{13} \mathrm{C} \mathrm{NMR}\left(126 \mathrm{MHz}, \mathrm{CDCl}_{3}\right) \delta 172.36,163.34,151.69,148.53,131.62,131.01,128.41$, 128.32, 127.07, 126.79, 126.34, 106.02, 103.45, 77.39, 29.76, 26.67. HRMS (ESI) calcd for $\mathrm{C}_{16} \mathrm{H}_{13} \mathrm{~N}_{3} \mathrm{O}_{4} \mathrm{Li}\left(\mathrm{MLi}^{+}\right)$318.1061, found 318.1064. 


\section{General irradiation/oxidation procedure $F$ for the synthesis of ketones 6ab and 11f-h:}

Approximately $0.03 \mathrm{M}$ solution of a photoprecursors $1 \mathbf{a b}$ or $\mathbf{9 f}-\mathbf{h}(1 \mathrm{mmol})$ in DMSO, was degassed by bubbling nitrogen for 30 min and irradiated with UV LED-based illuminator (five $250 \mathrm{~mW} @$ $365 \mathrm{~nm}$ Nichia UVLEDs) until the starting material was consumed. To the photo products DessMartin periodinane (DMP, $1.20 \mathrm{mmol}$ ) was added, and the mixture was stirred at room temperature for 1d. The reaction mixture was concentrated under vacuum, then the residue was dissolved in 60 $\mathrm{mL}$ of chloroform and thoroughly washed with saturated aq. $\mathrm{NaHCO}_{3}(10 \mathrm{~mL})$, saturated aq. $\mathrm{Na}_{2} \mathrm{~S}_{2} \mathrm{O}_{3}(10 \mathrm{~mL})$, and water, $2 \times 20 \mathrm{~mL}$. Combined aqueous layer was extracted with chloroform $(50 \mathrm{~mL})$. Combined organic layer was dried over anhydrous $\mathrm{Na}_{2} \mathrm{SO}_{4}$, and the solvent was removed under reduced pressure. The product was purified by flash column chromatography.

Irradiation/oxidation of 2a: General Procedure $\mathrm{F}$ was followed on $0.21 \mathrm{~g}(0.69 \mathrm{mmol})$ of photoprecursor 2a in $20 \mathrm{~mL}$ of DMSO, 3h irradiation. The product was purified by flash column chromatography which afforded:

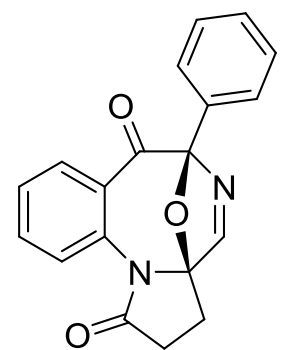

\section{9-phenyl-6,7-benzo-4,12-dioxa-5,10-diaza-tricyclo[7.2.1.0 $\left.{ }^{5,9}\right]$ dodeca-6,10-} diene-8-one (6a): $0.14 \mathrm{~g}(64 \%) .{ }^{1} \mathrm{H} \mathrm{NMR}\left(500 \mathrm{MHz}, \mathrm{CDCl}_{3}\right) \delta 7.85(\mathrm{dd}, J=8.0,1.7 \mathrm{~Hz}, 1 \mathrm{H})$, $7.76(\mathrm{dd}, J=8.2,1.2 \mathrm{~Hz}, 1 \mathrm{H}), 7.69-7.63(\mathrm{~m}, 2 \mathrm{H}), 7.59$ (ddd, $J=8.3,7.3,1.7 \mathrm{~Hz}, 1 \mathrm{H}), 7.56$ (s, $1 \mathrm{H}), 7.50-7.42$ (m, 3H), 7.32 (ddd, $J=8.0,7.3,1.2 \mathrm{~Hz}, 1 \mathrm{H}), 3.09-2.96$ (m, 1H), $2.86-2.73$ (m, 2H), $2.61-2.51(\mathrm{~m}, 1 \mathrm{H}) .{ }^{13} \mathrm{C} \mathrm{NMR}\left(126 \mathrm{MHz}, \mathrm{CDCl}_{3}\right) \delta 192.86,172.63,161.04,134.88$, 133.20, 132.76, 132.23, 129.32, 128.22, 127.63, 127.29, 126.82, 125.58, 110.71, 105.35, 29.84, 27.07. HRMS (ESI) calcd for $\mathrm{C}_{19} \mathrm{H}_{14} \mathrm{~N}_{2} \mathrm{O}_{3} \mathrm{Li}\left(\mathrm{MLi}^{+}\right) 325.1159$, found 325.1164 . 
<smiles>O=C(Nc1c2n(c3ccccc3c1=O)CCC2=O)c1ccccc1</smiles>

\section{N-(1,5-dioxo-1,2,3,5-tetrahydropyrrolo[1,2-a]quinolin-4-yl)}

benzamide (7a): $0.04 \mathrm{~g}(18 \%) .{ }^{1} \mathrm{H}$ NMR $\left(500 \mathrm{MHz}, \mathrm{CDCl}_{3}\right) \delta 9.20(\mathrm{dt}, J=8.7,0.8 \mathrm{~Hz}, 1 \mathrm{H})$, $8.42-8.33(\mathrm{~m}, 2 \mathrm{H}), 8.01-7.92(\mathrm{~m}, 2 \mathrm{H}), 7.77$ (ddd, $J=8.7,7.1,1.7 \mathrm{~Hz}, 1 \mathrm{H}), 7.61-7.47$ (m, $4 \mathrm{H}), 3.46-3.36(\mathrm{~m}, 2 \mathrm{H}), 3.05-2.92(\mathrm{~m}, 2 \mathrm{H}) .{ }^{13} \mathrm{C} \mathrm{NMR}\left(126 \mathrm{MHz}, \mathrm{CDCl}_{3}\right) \delta 175.92,175.01$, 165.50, 148.30, 136.21, 133.93, 133.48, 132.42, 128.93, 127.79, 126.53, 126.51, 124.03, 118.21, 118.16, 29.57, 23.28. HRMS (ESI) calcd for $\mathrm{C}_{19} \mathrm{H}_{15} \mathrm{~N}_{2} \mathrm{O}_{3} \mathrm{Li}\left(\mathrm{MLi}^{+}\right)$325.1159, found 325.1162.

Irradiation/oxidation of $\mathbf{2 b}$ : General Procedure F was followed on $0.21 \mathrm{~g}(0.60 \mathrm{mmol})$ of photoprecursor $\mathbf{2 b}$ in $20 \mathrm{~mL}$ of DMSO, 3h irradiation. The product was purified by flash column chromatography which afforded:<smiles>COc1ccc([C@]23N=C[C@@]4(CCC(=O)N4c4ccccc4C2=O)O3)cc1</smiles>

\section{9-(4-methoxyphenyl)-6,7-benzo-4,12-dioxa-5,10-diaza-tricyclo[7.2.1.0 $\left.{ }^{5,9}\right]$}

dodeca-6,10-diene-8-one (6b): $0.13 \mathrm{~g}(62 \%) .{ }^{1} \mathrm{H} \mathrm{NMR}\left(500 \mathrm{MHz}, \mathrm{CDCl}_{3}\right) \delta 7.84$ (dd, $J=8.0$, $1.7 \mathrm{~Hz}, 1 \mathrm{H}), 7.75(\mathrm{dd}, J=8.1,1.1 \mathrm{~Hz}, 1 \mathrm{H}), 7.61-7.55(\mathrm{~m}, 3 \mathrm{H}), 7.53(\mathrm{~s}, 1 \mathrm{H}), 7.34-7.28(\mathrm{~m}, 1 \mathrm{H})$, $7.02-6.97(\mathrm{~m}, 2 \mathrm{H}), 3.85(\mathrm{~s}, 3 \mathrm{H}), 3.08-2.95(\mathrm{~m}, 1 \mathrm{H}), 2.86-2.71(\mathrm{~m}, 2 \mathrm{H}), 2.59-2.49(\mathrm{~m}, 1 \mathrm{H})$. ${ }^{13} \mathrm{C} \mathrm{NMR}\left(126 \mathrm{MHz}, \mathrm{CDCl}_{3}\right) \delta 193.10,172.61,160.89,160.34,133.13,132.73,132.21,128.94$, 127.33, 127.01, 126.76, 125.53, 113.68, 110.57, 105.35, 55.47, 29.84, 27.08. HRMS (ESI) calcd for $\mathrm{C}_{20} \mathrm{H}_{17} \mathrm{~N}_{2} \mathrm{O}_{4}\left(\mathrm{MH}^{+}\right)$349.1183, found 349.1177 


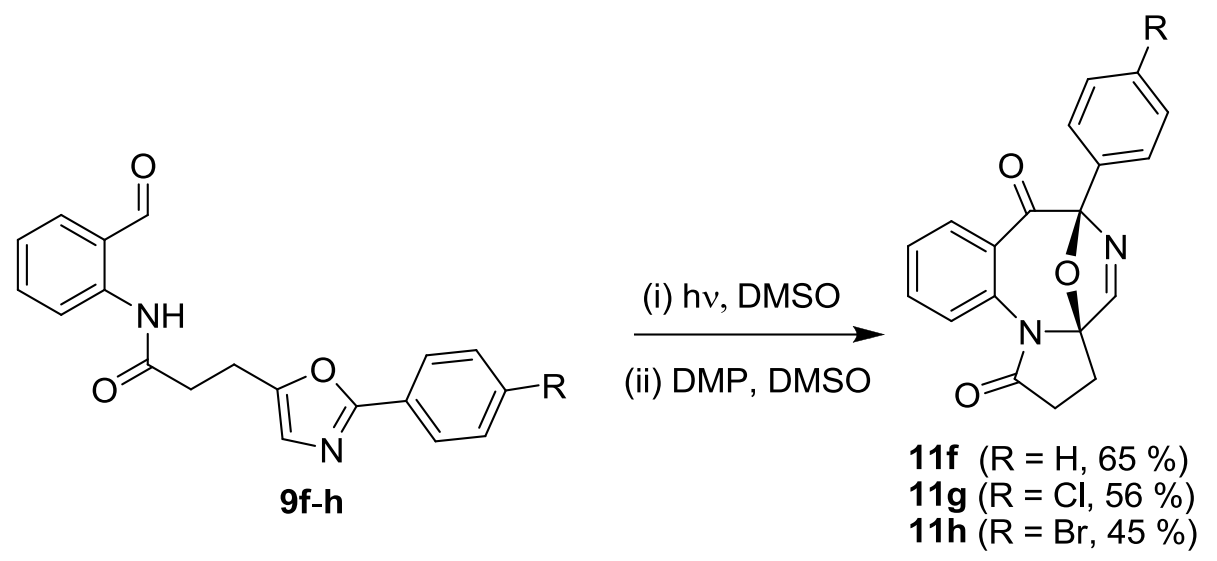<smiles>O=C1CC[C@@]23N=C[C@](c4ccccc4)(O2)C(=O)c2ccccc2N13</smiles>

9-phenyl-6,7-benzo-4,12-dioxa-5,11-diaza-tricyclo[7.2.1.0 $\left.{ }^{5,9}\right]$ dodeca-6,10-diene8-one (11f): General Procedure F was followed on $0.31 \mathrm{~g}(0.97 \mathrm{mmol})$ of photoprecursor $9 f$ in 20 $\mathrm{mL}$ of DMSO, 5h 30min irradiation. The product was purified by flash column chromatography which afforded: $0.20 \mathrm{~g}(65 \%)$ of imine 11f. ${ }^{1} \mathrm{H}$ NMR $\left(500 \mathrm{MHz}, \mathrm{CDCl}_{3}\right) \delta 8.01(\mathrm{~s}, 1 \mathrm{H}), 7.93$ (dd, $J=8.0,1.7 \mathrm{~Hz}, 1 \mathrm{H}), 7.91(\mathrm{dd}, J=8.3,1.2 \mathrm{~Hz}, 1 \mathrm{H}), 7.63(\mathrm{ddd}, J=8.3,7.2,1.7 \mathrm{~Hz}, 1 \mathrm{H}), 7.52-$ $7.42(\mathrm{~m}, 5 \mathrm{H}), 7.32(\mathrm{ddd}, J=8.0,7.3,1.2 \mathrm{~Hz}, 1 \mathrm{H}), 3.12-2.91(\mathrm{~m}, 3 \mathrm{H}), 2.78-2.69(\mathrm{~m}, 1 \mathrm{H}) .{ }^{13} \mathrm{C}$ NMR $\left(126 \mathrm{MHz}, \mathrm{CDCl}_{3}\right) \delta 191.87,172.22,166.63,134.01,132.54,132.30,132.00,129.14$, 128.67, 128.49, 126.76, 126.33, 126.23, 122.09, 96.26, 30.70, 29.77. HRMS (ESI) calcd for $\mathrm{C}_{19} \mathrm{H}_{14} \mathrm{~N}_{2} \mathrm{O}_{3} \mathrm{Li}\left(\mathrm{MLi}^{+}\right)$325.1159, found 325.1167.<smiles>O=C1CCC23N=CC(c4ccc(Cl)cc4)(O2)N2CC1(O3)C(=O)c1ccccc12</smiles>

\section{9-(4-Chlorophenyl)-6,7-benzo-4,12-dioxa-5,11-diaza-tricyclo[7.2.1.0 $\left.{ }^{5,9}\right]$}

dodeca-6,10-diene-8-one (11g): General Procedure F followed on $0.20 \mathrm{~g}(0.56 \mathrm{mmol})$ of photoprecursor $\mathbf{9 g}$ in $20 \mathrm{~mL}$ of DMSO, $4 \mathrm{~h}$ irradiation. The product was purified by flash column 
chromatography which afforded $0.11 \mathrm{~g}(56 \%)$ of imine $11 \mathrm{~g} .{ }^{1} \mathrm{H}$ NMR $\left(500 \mathrm{MHz}, \mathrm{CDCl}_{3}\right) \delta 7.96$ (s, $1 \mathrm{H}), 7.93(\mathrm{dd}, J=8.0,1.7 \mathrm{~Hz}, 1 \mathrm{H}), 7.90(\mathrm{dd}, J=8.3,1.2 \mathrm{~Hz}, 1 \mathrm{H}), 7.64(\mathrm{ddd}, J=8.3,7.3,1.7$ $\mathrm{Hz}, 1 \mathrm{H}), 7.49-7.44$ (m, 2H), $7.41-7.37$ (m, 2H), 7.32 (ddd, $J=8.3,7.3,1.2 \mathrm{~Hz}, 1 \mathrm{H}), 3.14-2.92$ (m, 4H), $2.77-2.68(\mathrm{~m}, 1 \mathrm{H}) .{ }^{13} \mathrm{C}$ NMR $\left(126 \mathrm{MHz}, \mathrm{CDCl}_{3}\right) \delta 191.55,172.17,166.14,135.30$, $134.21,132.54,132.34,130.49,128.94,128.28,128.20,126.43,126.34,122.23,95.84,30.65$, 29.75. HRMS (ESI) calcd for $\mathrm{C}_{19} \mathrm{H}_{13} \mathrm{ClLiN}_{2} \mathrm{O}_{3}\left(\mathrm{MLi}^{+}\right)$359.0769, found 359.0773 (X-ray - see cif)

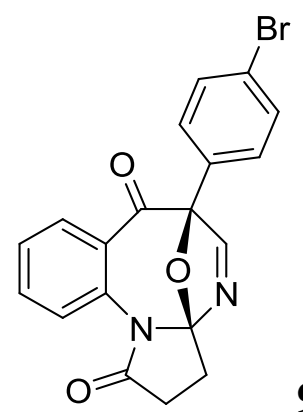

\section{9-(4-Bromophenyl)-6,7-benzo-4,12-dioxa-5,11-diaza-}

tricyclo[7.2.1.0 ${ }^{5,9}$ ]dodeca-6,10-diene-8-one (4h): General Procedure F followed on $0.29 \mathrm{~g}(0.72$ $\mathrm{mmol}$ ) of aldehyde (1h) in $20 \mathrm{~mL}$ of DMSO, and irradiated for $6 \mathrm{~h}$. The product was purified by flash column chromatography which afforded $0.13 \mathrm{~g}$ (45 \%). of imine 4h. ${ }^{1} \mathrm{H}$ NMR (500 MHz, $\left.\mathrm{CDCl}_{3}\right) \delta 7.95(\mathrm{~s}, 1 \mathrm{H}), 7.92(\mathrm{dd}, J=8.0,1.7 \mathrm{~Hz}, 1 \mathrm{H}), 7.89(\mathrm{dd}, J=8.3,1.2 \mathrm{~Hz}, 1 \mathrm{H}), 7.67-7.59$ (m, 3H), $7.36-7.29(\mathrm{~m}, 4 \mathrm{H}), 3.19-2.88(\mathrm{~m}, 4 \mathrm{H}), 2.73(\mathrm{ddd}, J=11.9,8.5,3.8 \mathrm{~Hz}, 1 \mathrm{H}) .{ }^{13} \mathrm{C}$ NMR $\left(126 \mathrm{MHz}, \mathrm{CDCl}_{3}\right) \delta 191.44,172.15,166.07,134.19,132.51,132.32,131.85,131.00,128.47$, $128.25,126.41,126.32,123.48,122.21,95.86,30.63,29.72$. HRMS (ESI) calcd for $\mathrm{C}_{19} \mathrm{H}_{13} \mathrm{BrLiN}_{2} \mathrm{O}_{3}\left(\mathrm{MLi}^{+}\right)$403.0264, found 403.0273. 


\section{POSTPHOTOCHEMICAL TRANSFORMATIONS}

In situ generation of dibromide 12.

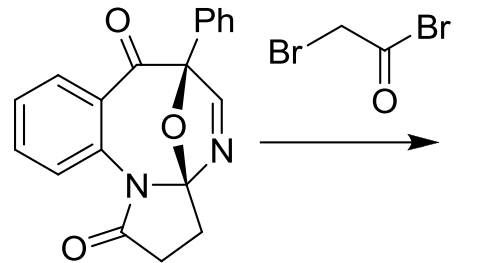

$11 f$

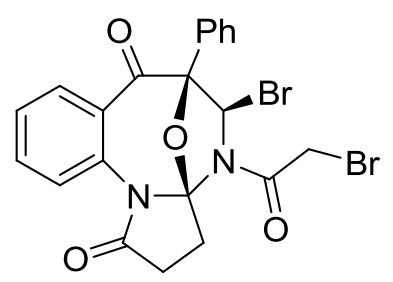

12<smiles>O=C1CC2[C@]3(CCC(=O)N3c3ccccc3)OC(=O)[C@@]2(c2ccccc2)O1</smiles>

2-bromo-1-(9-phenyl-10-bromo-6,7-benzo-4,12-dioxa-5,11-

diazatricyclo[7.2.1.0 ${ }^{1,5}$ ]dodeca-6-ene-8-one-11-yl)ethanone (12): Imine $11 f(0.33 \mathrm{mmol})$ was taken up in $5 \mathrm{~mL}$ of $\mathrm{CH}_{2} \mathrm{Cl}_{2}$ and treated with bromoacetyl bromide $(0.07 \mathrm{~g}, 0.35 \mathrm{mmol}, 30.7 \mu \mathrm{L})$ at room temperature under nitrogen atmosphere. The reaction mixture was stirred at room temperature for $3 \mathrm{~h}$ which afforded dibromide 12 quantitatively (by $\left.{ }^{1} \mathrm{H} \mathrm{NMR}\right) .{ }^{1} \mathrm{H} \mathrm{NMR}(500 \mathrm{MHz}$, $\left.\mathrm{CDCl}_{3}\right) \delta 8.44(\mathrm{dd}, J=8.5,1.1 \mathrm{~Hz}, 1 \mathrm{H}), 7.77(\mathrm{dd}, J=8.0,1.7 \mathrm{~Hz}, 1 \mathrm{H}), 7.60(\mathrm{ddd}, J=8.7,7.2,1.7$ $\mathrm{Hz}, 1 \mathrm{H}), 7.55-7.48(\mathrm{~m}, 2 \mathrm{H}), 7.49-7.41(\mathrm{~m}, 3 \mathrm{H}), 7.22(\mathrm{ddd}, J=8.2,7.2,1.1 \mathrm{~Hz}, 1 \mathrm{H}), 6.77$ (s, 1H), 4.11 (d, $J=11.5 \mathrm{~Hz}, 1 \mathrm{H}), 3.79$ (d, $J=11.5 \mathrm{~Hz}, 1 \mathrm{H}), 3.08$ (ddd, $J=15.5,11.0,5.8 \mathrm{~Hz}, 1 \mathrm{H}$ ), $2.99-2.87(\mathrm{~m}, 2 \mathrm{H}), 2.83(\mathrm{ddd}, J=15.4,11.0,5.8 \mathrm{~Hz}, 1 \mathrm{H}) .{ }^{13} \mathrm{C} \mathrm{NMR}\left(126 \mathrm{MHz}, \mathrm{CDCl}_{3}\right) \delta 196.04$, $172.97,164.35,134.90,134.29,133.91,132.73,129.33,128.09,127.17,127.14,125.59,122.59$, 109.11, 93.31, 68.93, 31.10, 30.78, 26.01.

\section{General Procedure G for the synthesis of fused N,S,O heterocycles 13-15:}

Dibromide 12 was generated as described above $(0.33 \mathrm{mmol})$. To this reaction mixture pyridine (0.99 mmol, 3 eq.) and a dinucleophile $(0.33 \mathrm{mmol})$ were added and stirred at room temperature overnight. The solvent was removed in vacuum and the residue was purified by flash column chromatography. 


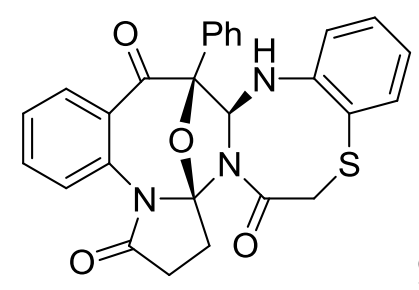

\section{9-phenyl-(6,7),(12,13)-dibenzo-14-thia-4,16,18-trioxa-5,11,17-triaza-}

pentacyclo[8.7.1.0 $\left.{ }^{1,5} \cdot 0^{10,17}\right]$ octadec-6,12-diene-8-one (14): General procedure $\mathbf{G}$ was followed on a $0.44 \mathrm{mmol}$ scale of imine $11 \mathrm{f}$ to give $0.20 \mathrm{~g}(94 \%)$ of thiadiazacane $14 .{ }^{1} \mathrm{H} \mathrm{NMR}(500 \mathrm{MHz}$, $\left.\mathrm{CDCl}_{3}\right) \delta 8.18(\mathrm{~d}, J=8.4 \mathrm{~Hz}, 1 \mathrm{H}), 7.76(\mathrm{dd}, J=7.9,1.7 \mathrm{~Hz}, 1 \mathrm{H}), 7.60(\mathrm{ddd}, J=8.4,7.3,1.7 \mathrm{~Hz}$, 1H), $7.52(\mathrm{dt}, J=4.1,1.4 \mathrm{~Hz}, 3 \mathrm{H}), 7.45(\mathrm{ddd}, J=6.2,2.7,1.5 \mathrm{~Hz}, 2 \mathrm{H}), 7.32-7.26(\mathrm{~m}, 2 \mathrm{H}), 7.06$ $(\mathrm{td}, J=7.7,1.6 \mathrm{~Hz}, 1 \mathrm{H}), 6.92(\mathrm{~s}, 1 \mathrm{H}), 6.84(\mathrm{td}, J=7.5,1.3 \mathrm{~Hz}, 1 \mathrm{H}), 6.04(\mathrm{dd}, J=8.0,1.3 \mathrm{~Hz}, 1 \mathrm{H})$, $3.85(\mathrm{~d}, J=15.4 \mathrm{~Hz}, 1 \mathrm{H}), 3.68$ (d, $J=8.3 \mathrm{~Hz}, 1 \mathrm{H}), 3.53$ (d, $J=15.5 \mathrm{~Hz}, 1 \mathrm{H}), 3.08-2.92(\mathrm{~m}, 2 \mathrm{H})$, $2.87(\mathrm{ddd}, J=15.7,10.2,4.5 \mathrm{~Hz}, 1 \mathrm{H}), 2.63(\mathrm{ddd}, J=13.3,10.2,4.7 \mathrm{~Hz}, 1 \mathrm{H}) .{ }^{13} \mathrm{C} \mathrm{NMR}(126 \mathrm{MHz}$, $\left.\mathrm{CDCl}_{3}\right) \delta 196.03,172.96,166.93,145.03,134.99,134.11,133.35,132.35,132.03,129.70,129.58$, $128.77,128.52$, 128.29, 125.96, 124.22, 122.37, 121.71, 120.55, 108.51, 91.34, 73.47, 37.80, 31.04, 29.81. HRMS (ESI) calcd for $\mathrm{C}_{27} \mathrm{H}_{22} \mathrm{~N}_{3} \mathrm{O}_{4} \mathrm{~S}\left(\mathrm{MH}^{+}\right)$484.1326, found 484.1320.

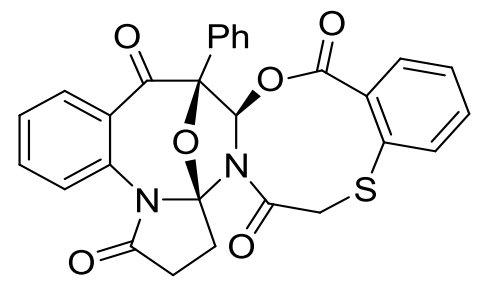

9-phenyl-(6,7), (13,14)-dibenzo-15-thia-4,11,17,19-tetraoxa-5,18diaza-pentacyclo[9.7.1.0 $\left.{ }^{1,5} \cdot .^{10,18}\right]$ nonadec-6,13-diene-8,12-dione (15): General procedure $\mathbf{G}$ was followed on a $0.43 \mathrm{mmol}$ scale of imine $11 \mathrm{f}$ to give $0.11 \mathrm{~g}(49 \%)$ of $10 .{ }^{1} \mathrm{H} \mathrm{NMR} \mathrm{(500} \mathrm{MHz,}$ $\left.\mathrm{CDCl}_{3}\right) \delta 8.23(\mathrm{~d}, J=8.4 \mathrm{~Hz}, 1 \mathrm{H}), 7.85(\mathrm{dd}, J=7.9,1.7 \mathrm{~Hz}, 1 \mathrm{H}), 7.61(\mathrm{ddd}, J=8.6,7.2,1.7 \mathrm{~Hz}$, 1H), $7.56-7.50(\mathrm{~m}, 2 \mathrm{H}), 7.47-7.33(\mathrm{~m}, 7 \mathrm{H}), 7.30(\mathrm{ddd}, J=8.2,7.2,1.1 \mathrm{~Hz}, 1 \mathrm{H}), 6.49(\mathrm{~s}, 1 \mathrm{H})$, $3.69(\mathrm{~d}, J=14.3 \mathrm{~Hz}, 1 \mathrm{H}), 3.15(\mathrm{~d}, J=14.3 \mathrm{~Hz}, 1 \mathrm{H}), 3.13-3.03(\mathrm{~m}, 2 \mathrm{H}), 2.98-2.88(\mathrm{~m}, 1 \mathrm{H})$, $2.87-2.77(\mathrm{~m}, 1 \mathrm{H}) .{ }^{13} \mathrm{C}$ NMR $\left(126 \mathrm{MHz}, \mathrm{CDCl}_{3}\right) \delta 194.77,172.84,167.67,167.34,134.75$, $134.48,133.44,132.77,132.75,132.64,131.19,131.06,131.02,129.19,128.52$, 128.36, 128.02, 127.16, 126.02, 123.97, 109.35, 90.91, 87.25, 39.58, 31.07, 29.37. HRMS (ESI) calcd for $\mathrm{C}_{28} \mathrm{H}_{21} \mathrm{~N}_{2} \mathrm{O}_{6} \mathrm{~S}\left(\mathrm{MH}^{+}\right)$513.1115, found 513.1120. (X-ray - see cif) 


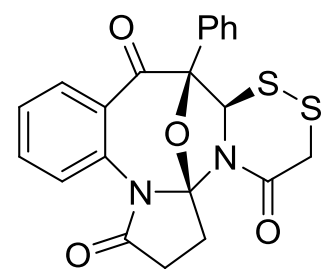

\section{9-phenyl-(6,7)-benzo-11,12-dithia-4,15,16-trioxa-5,15-diaza-pentacyclo}

[7.6.1.0 ${ }^{1,5} .^{10,15}$ ]hexadec-6-ene-8-one (13): Dibromide $12(0.070 \mathrm{~m}, 0.13 \mathrm{mmol})$ was generated in $12 \mathrm{~mL}$ of DCM and pyridine (74 $\mu \mathrm{L}, 4.5$ eq.) was added. Gaseous $\mathrm{H}_{2} \mathrm{~S}$ was bubbled for $1 \mathrm{~h}$ at $-40^{\circ} \mathrm{C}$, after which the reaction mixture was kept in the freezer $\left(-20^{\circ} \mathrm{C}\right)$ overnight and stirred at room temperature for 1 day (open air). Solvent was removed in vacuum and the residue was purified by flash column chromatography giving disulfide $130.023 \mathrm{~g} \mathrm{(42 \% ).}{ }^{1} \mathrm{H} \mathrm{NMR}(500 \mathrm{MHz}$, $\mathrm{CDCl}_{3} \delta 7.93(\mathrm{ddd}, J=8.2,5.0,1.4 \mathrm{~Hz}, 2 \mathrm{H}), 7.63-7.57(\mathrm{~m}, 3 \mathrm{H}), 7.49-7.43(\mathrm{~m}, 3 \mathrm{H}), 7.32$ (ddd, $J=8.2,7.3,1.2 \mathrm{~Hz}, 1 \mathrm{H}), 5.60(\mathrm{~s}, 1 \mathrm{H}), 3.40(\mathrm{~d}, J=17.6 \mathrm{~Hz}, 1 \mathrm{H}), 3.25$ (d, $J=17.6 \mathrm{~Hz}, 1 \mathrm{H}), 3.16-$ $2.99(\mathrm{~m}, 2 \mathrm{H}), 2.97-2.87(\mathrm{~m}, 1 \mathrm{H}), 2.65(\mathrm{ddd}, J=13.0,9.9,3.6 \mathrm{~Hz}, 1 \mathrm{H}) .{ }^{13} \mathrm{C} \mathrm{NMR}(126 \mathrm{MHz}$, $\left.\mathrm{CDCl}_{3}\right) \delta 192.42,172.56,162.10,135.12,134.07,132.81,132.69,130.69,129.37,128.45,126.51$, 126.48, 125.40, 110.21, 88.18, 73.47, 35.72, 30.52, 29.96. HRMS (ESI) calcd for $\mathrm{C}_{21} \mathrm{H}_{17} \mathrm{~N}_{2} \mathrm{O}_{4} \mathrm{~S}_{2}$ $\left(\mathrm{MH}^{+}\right)$425.0624, found 425.0623. 


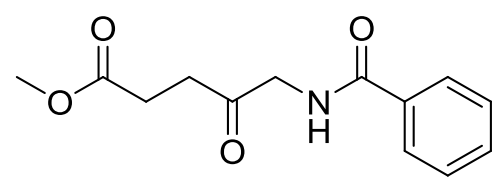

S1a

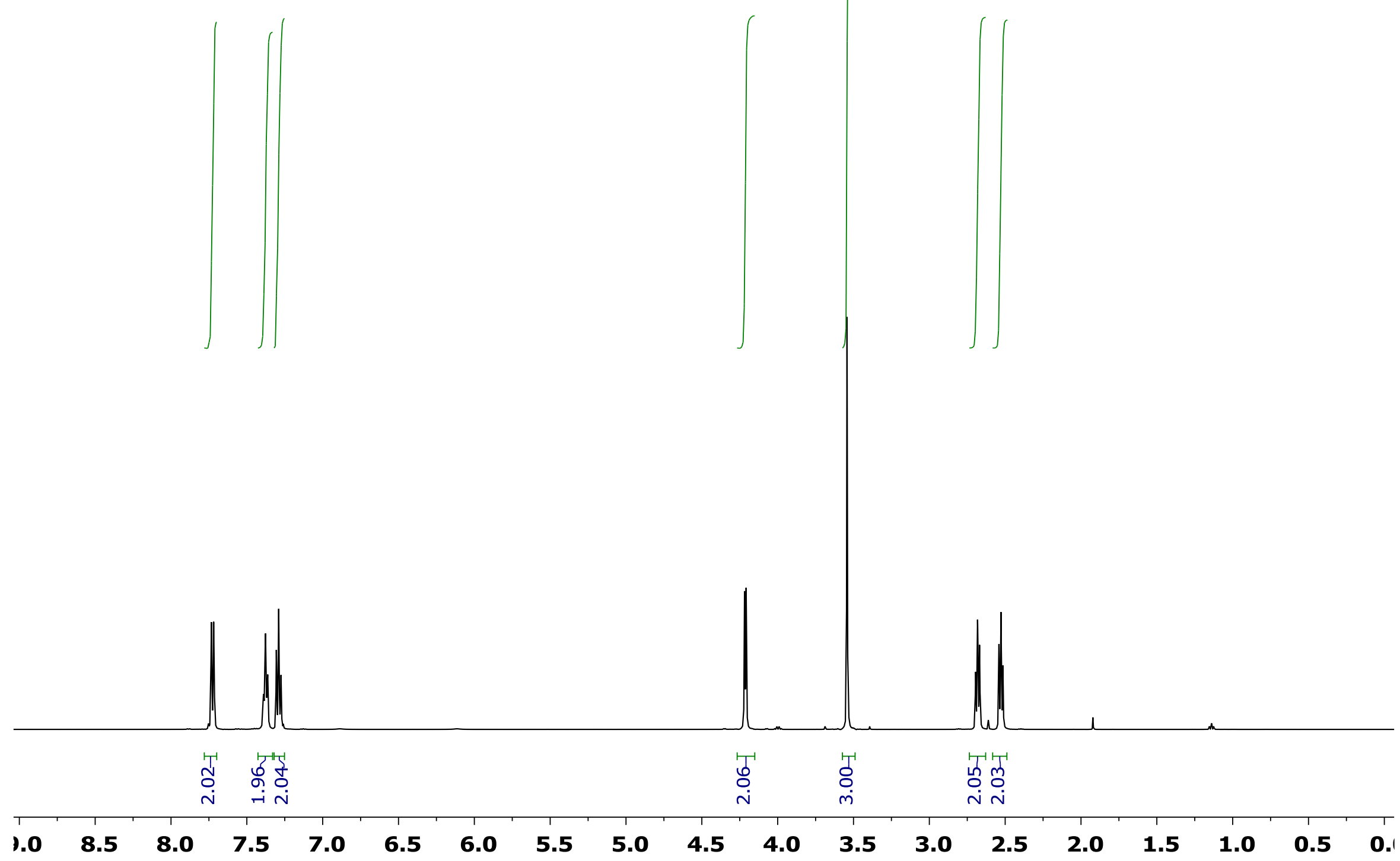




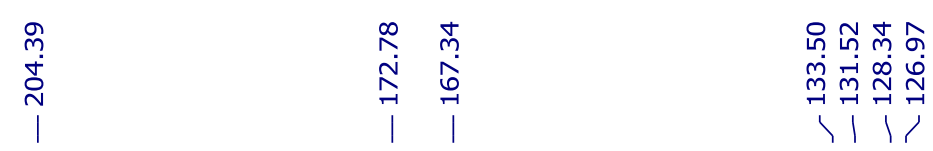

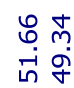

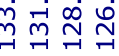

111

11

$\stackrel{\substack{m \\ \dot{m}}}{\stackrel{N}{N}}$

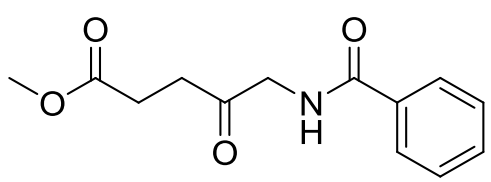

S1a

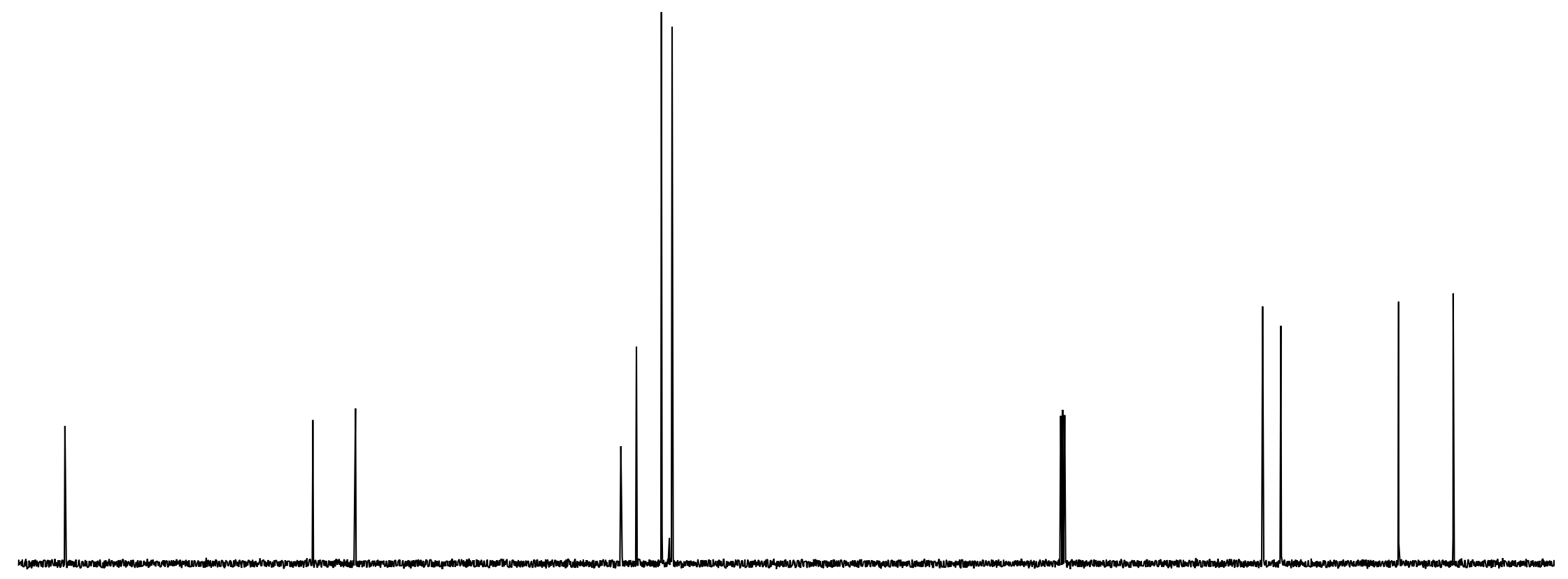

10200 
Photoassisted Diversity-Oriented Synthesis: Intramolecular Cycloadditions...

N.N. Bhuvan Kumar and Andrei G. Kutateladze, S31<smiles>COC(=O)CCC(=O)CNC(=O)c1ccc(OC)cc1</smiles>

S1b
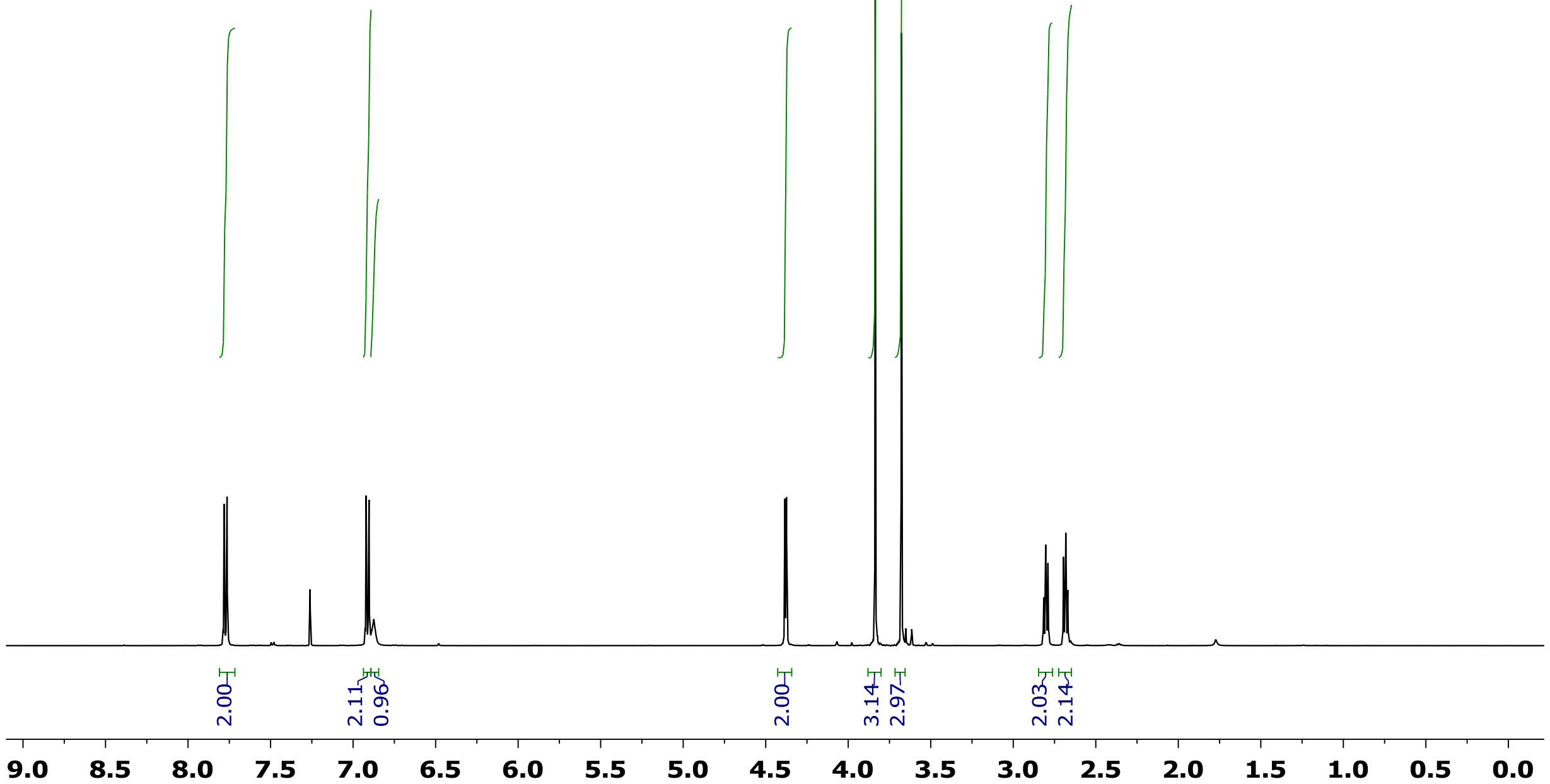
Photoassisted Diversity-Oriented Synthesis: Intramolecular Cycloadditions...

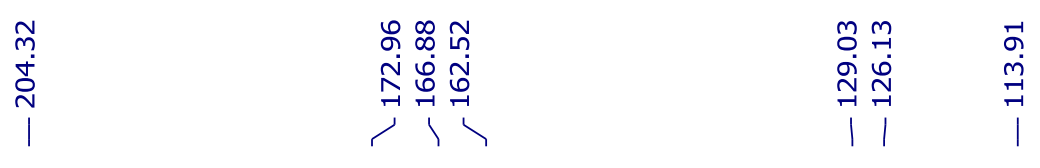<smiles>COC(=O)CCC(=O)CNC(=O)c1ccc(OC)cc1</smiles>

S1b
N.N. Bhuvan Kumar and Andrei G. Kutateladze, S32
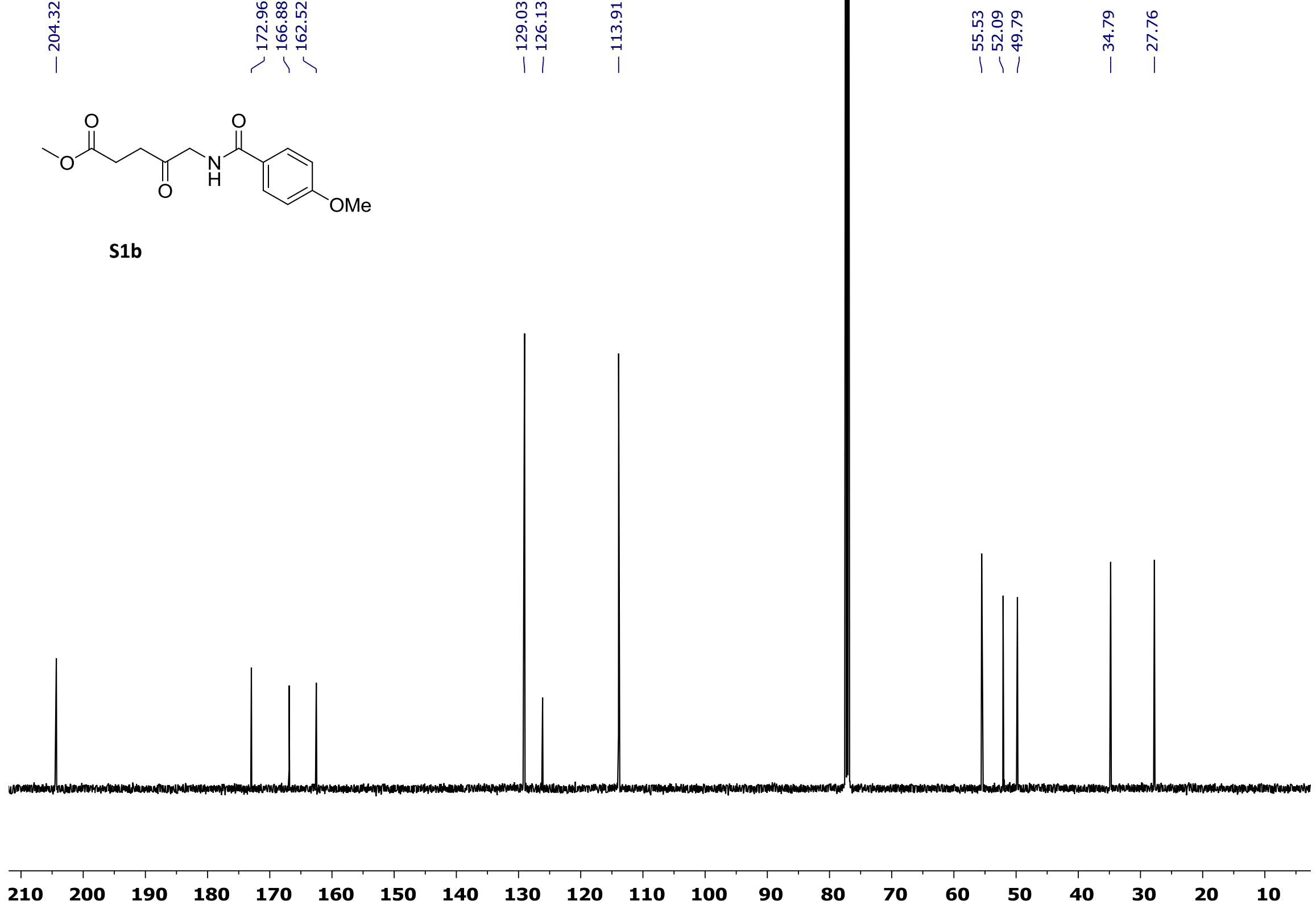
<smiles>COC(=O)CCC(=O)CNC(=O)c1ccco1</smiles>

S1c
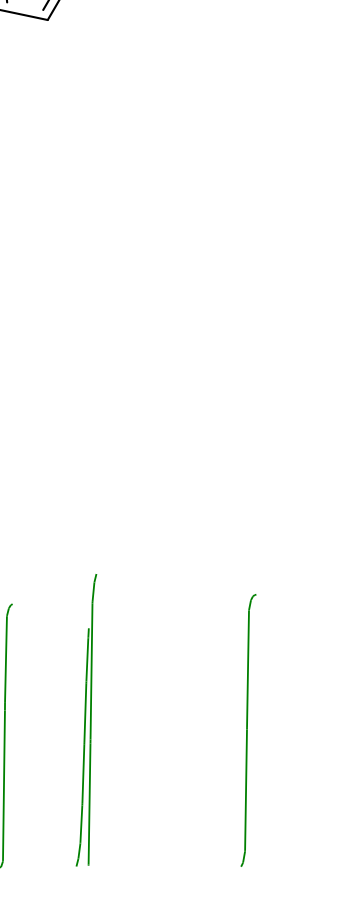


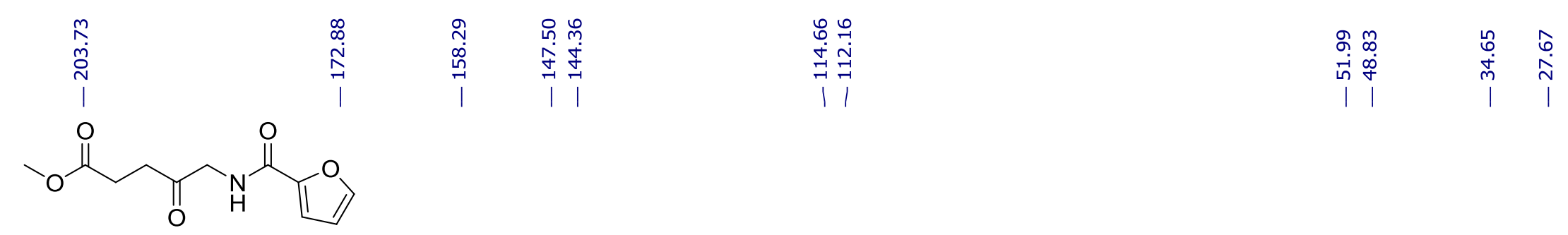

S1c

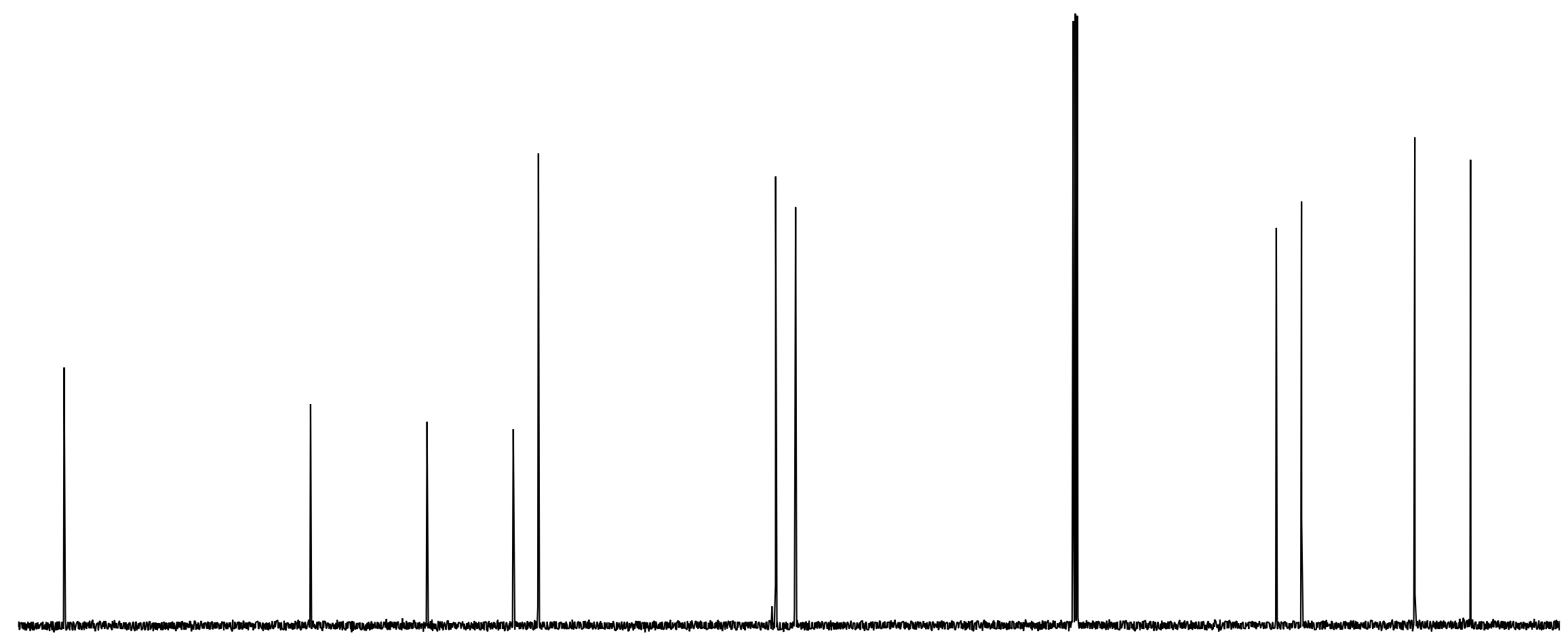




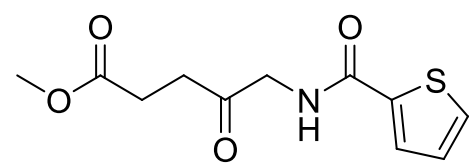

S1d
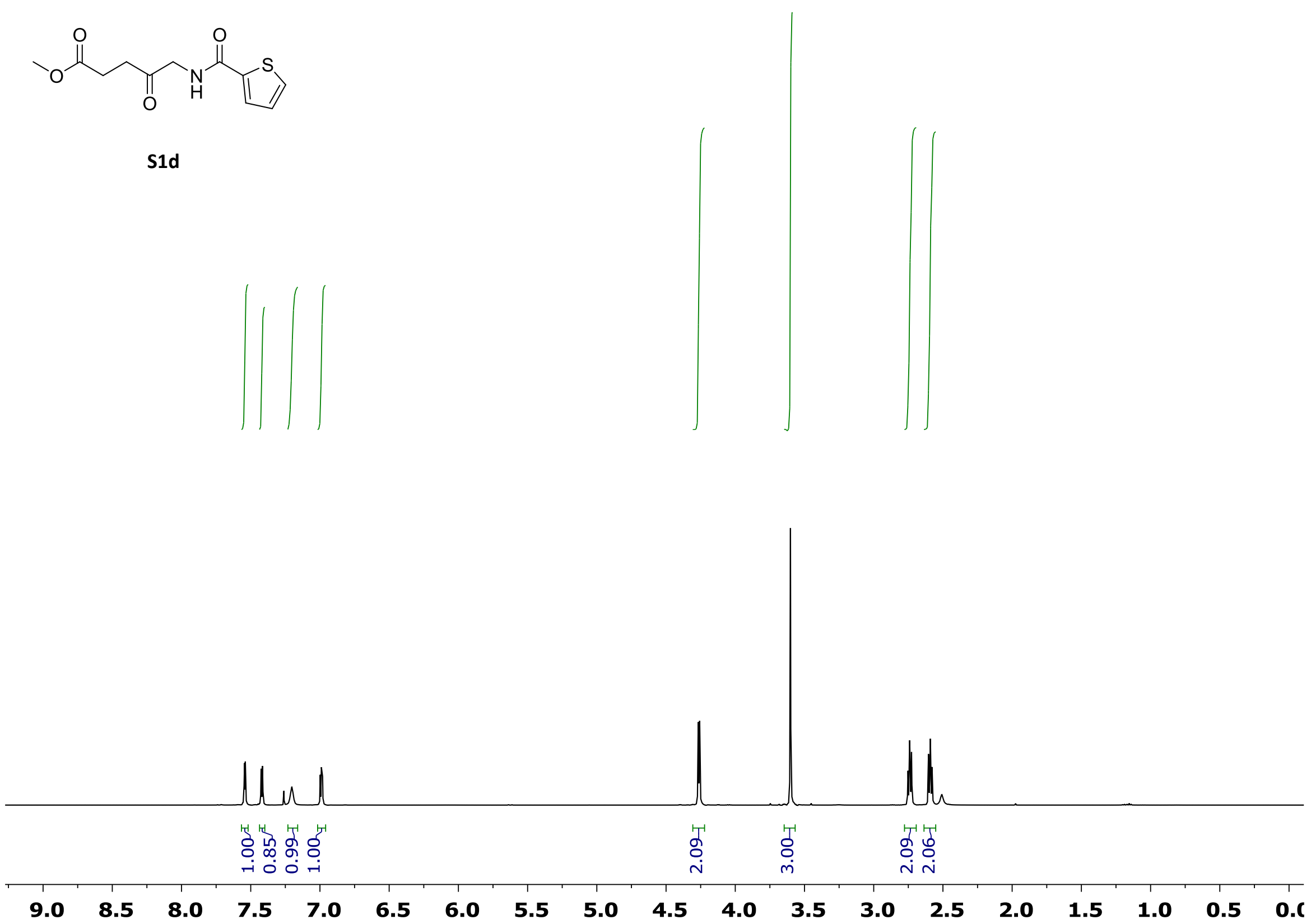
<smiles>COC(=O)CCC(=O)CNC(=O)c1cccs1</smiles>

S1d

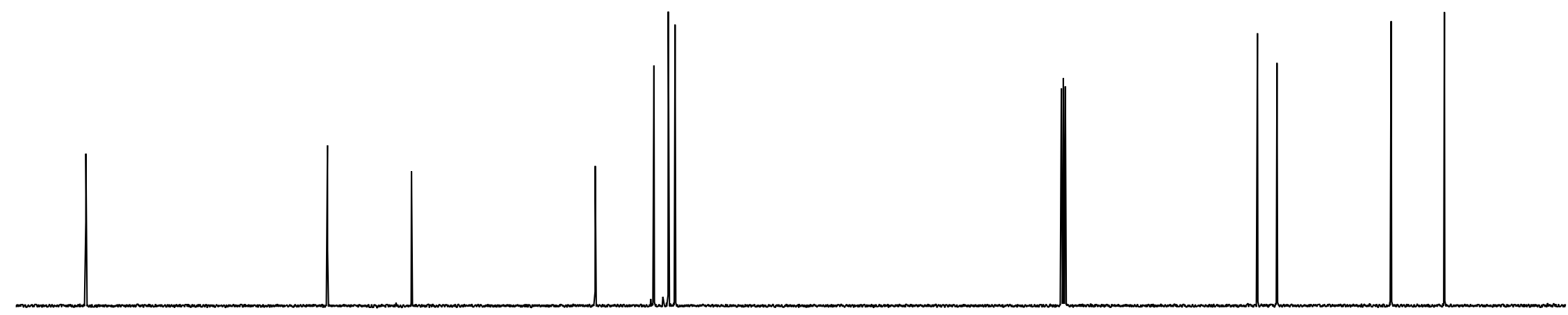




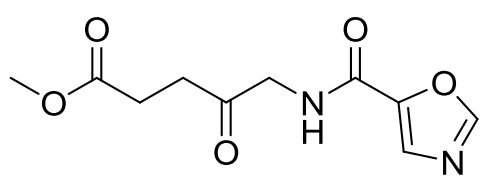

S1e
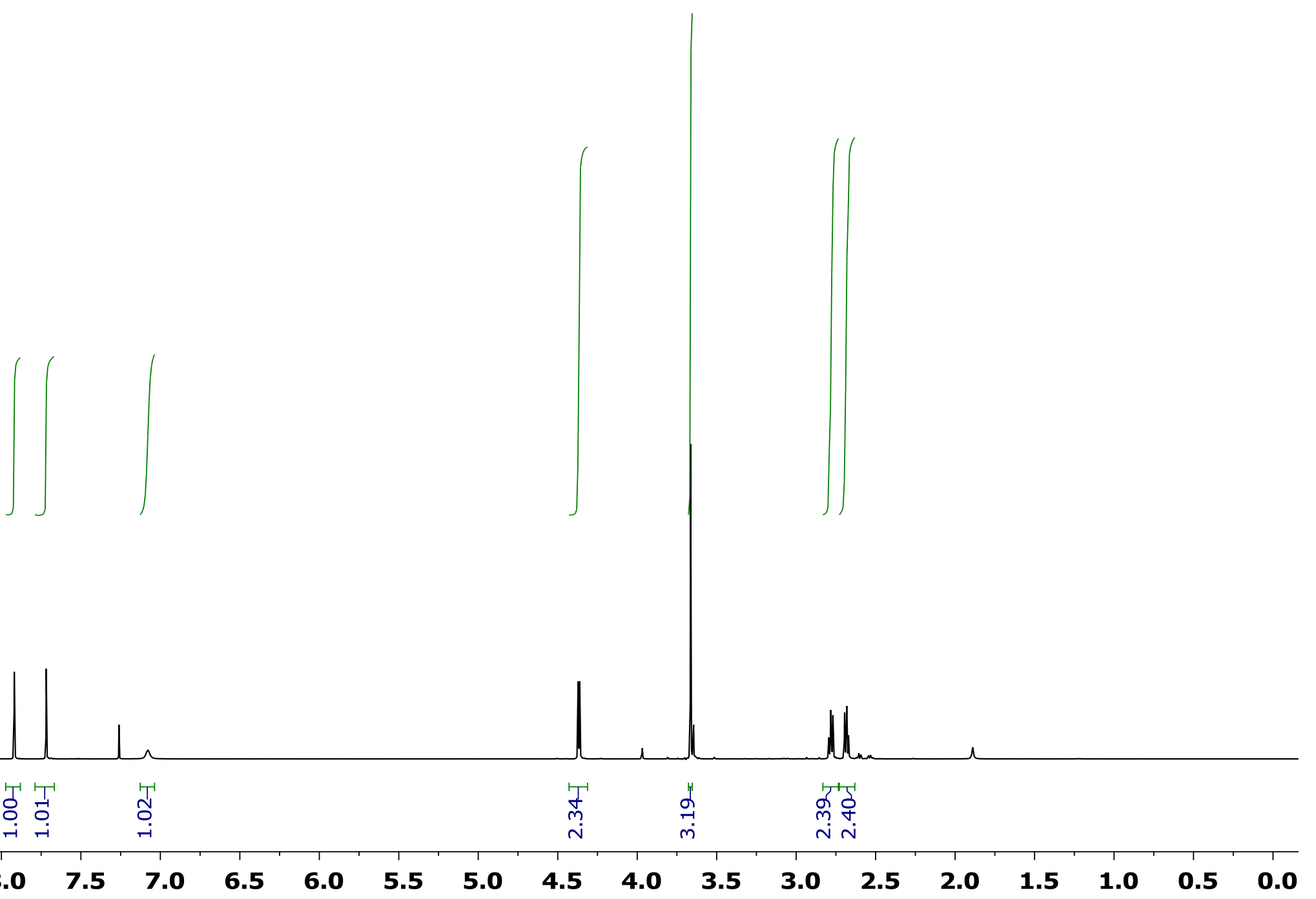

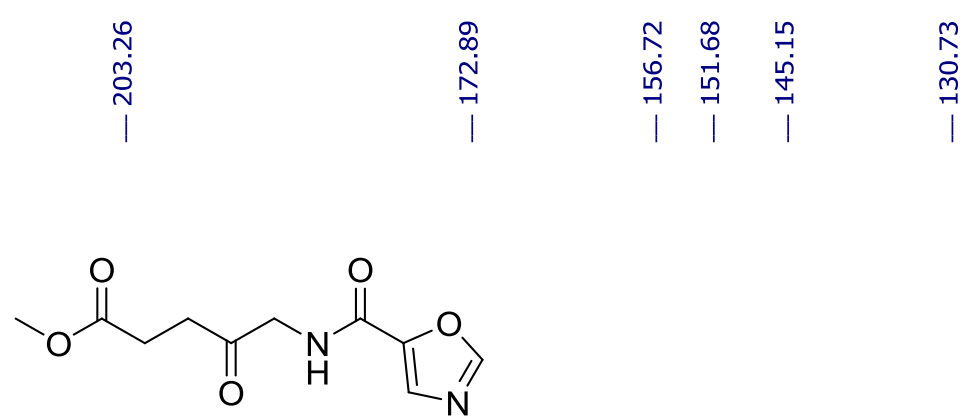

S1e

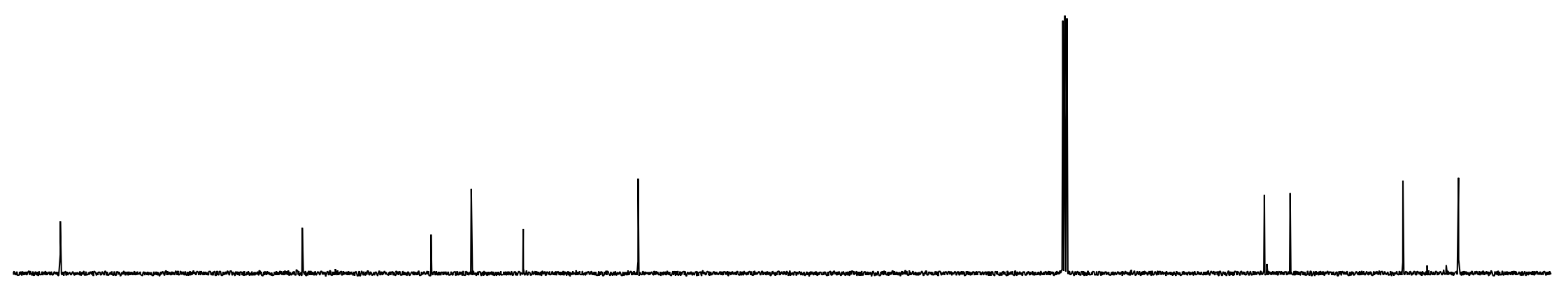

200

$\begin{array}{lllllll}190 & 180 & 170 & 160 & 150 & 140 & 130\end{array}$

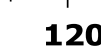

110100

90

80

70

6050

$50 \quad 40 \quad 30$ 
<smiles>COC(=O)CCc1cnc(-c2ccccc2)o1</smiles>

S2a

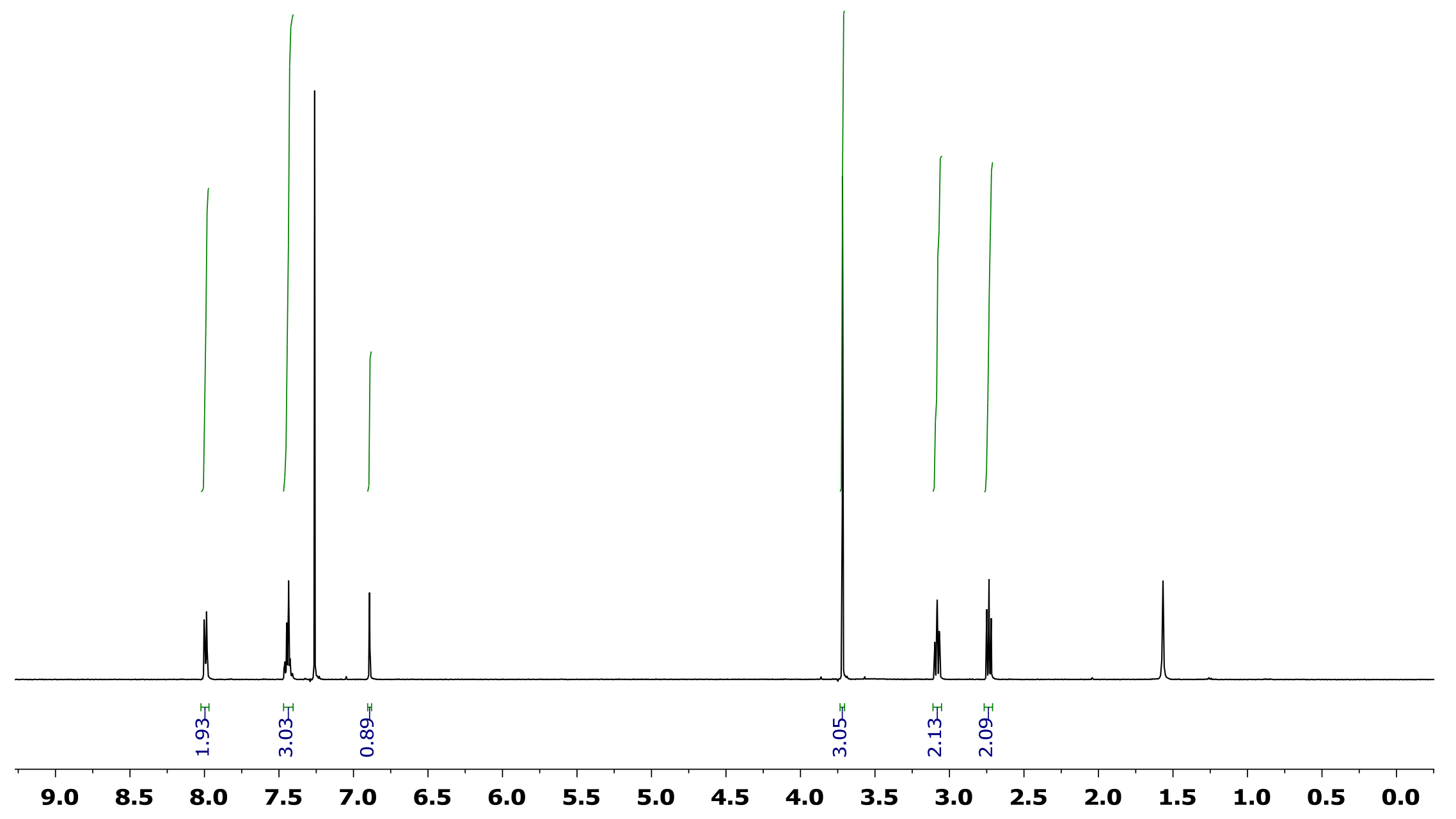




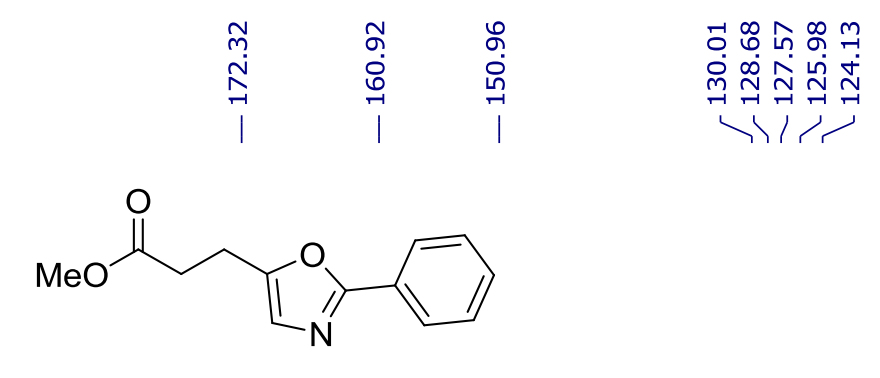

S2a
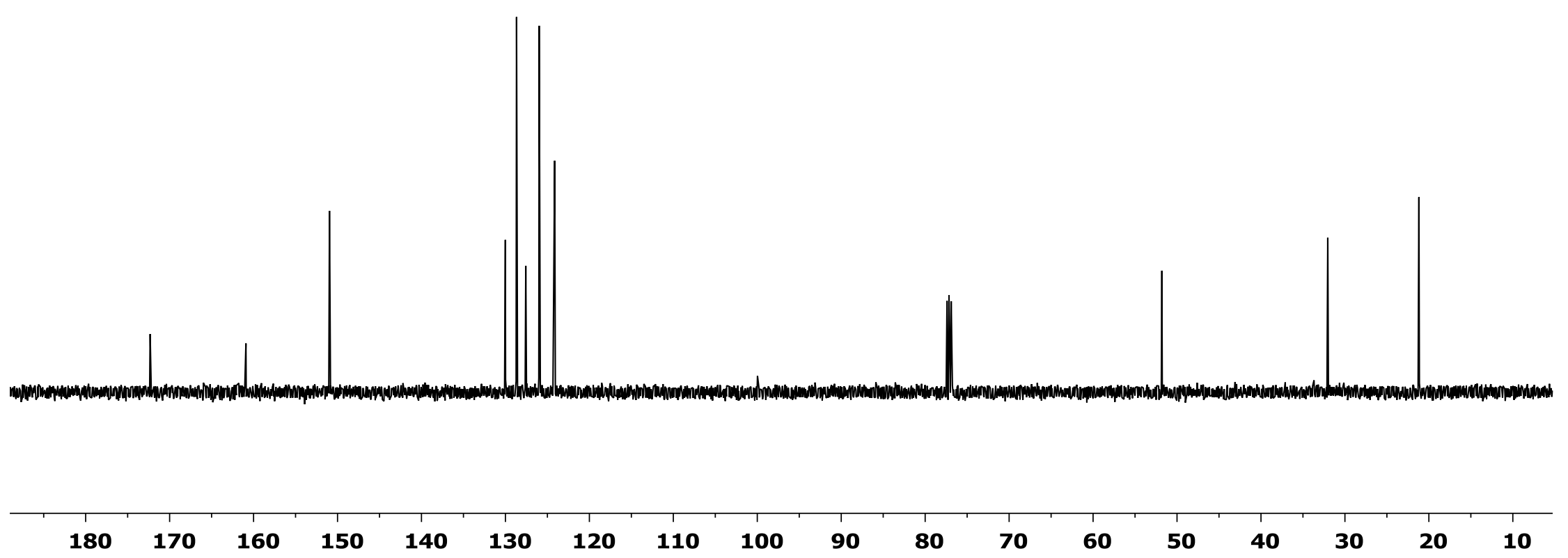
<smiles>COC(=O)CCc1cnc(-c2ccc(OC)cc2)o1</smiles>

S2b

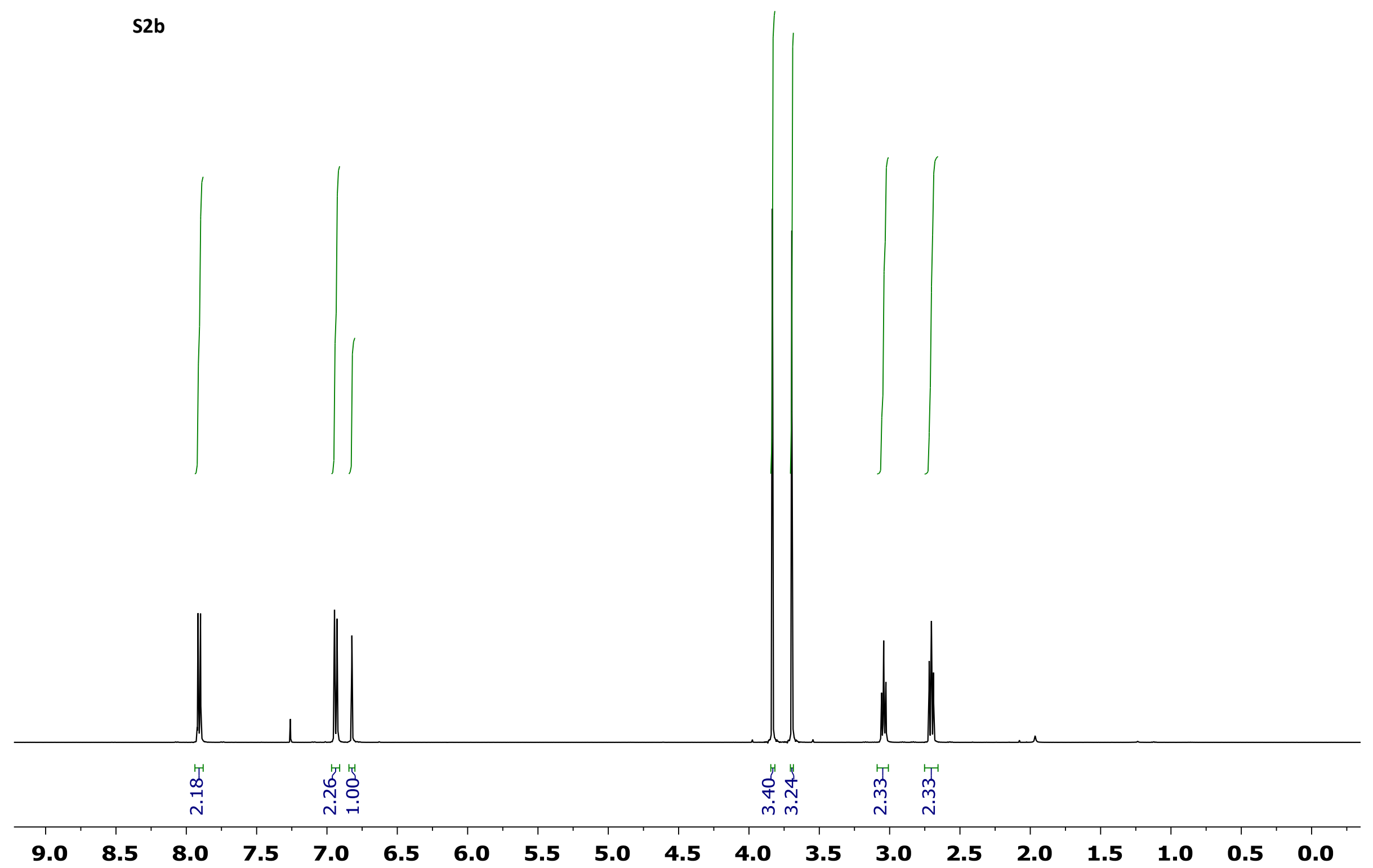




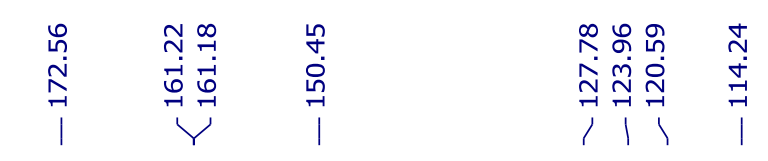<smiles>COC(=O)CCc1cnc(-c2ccc(OC)cc2)o1</smiles>

S2b

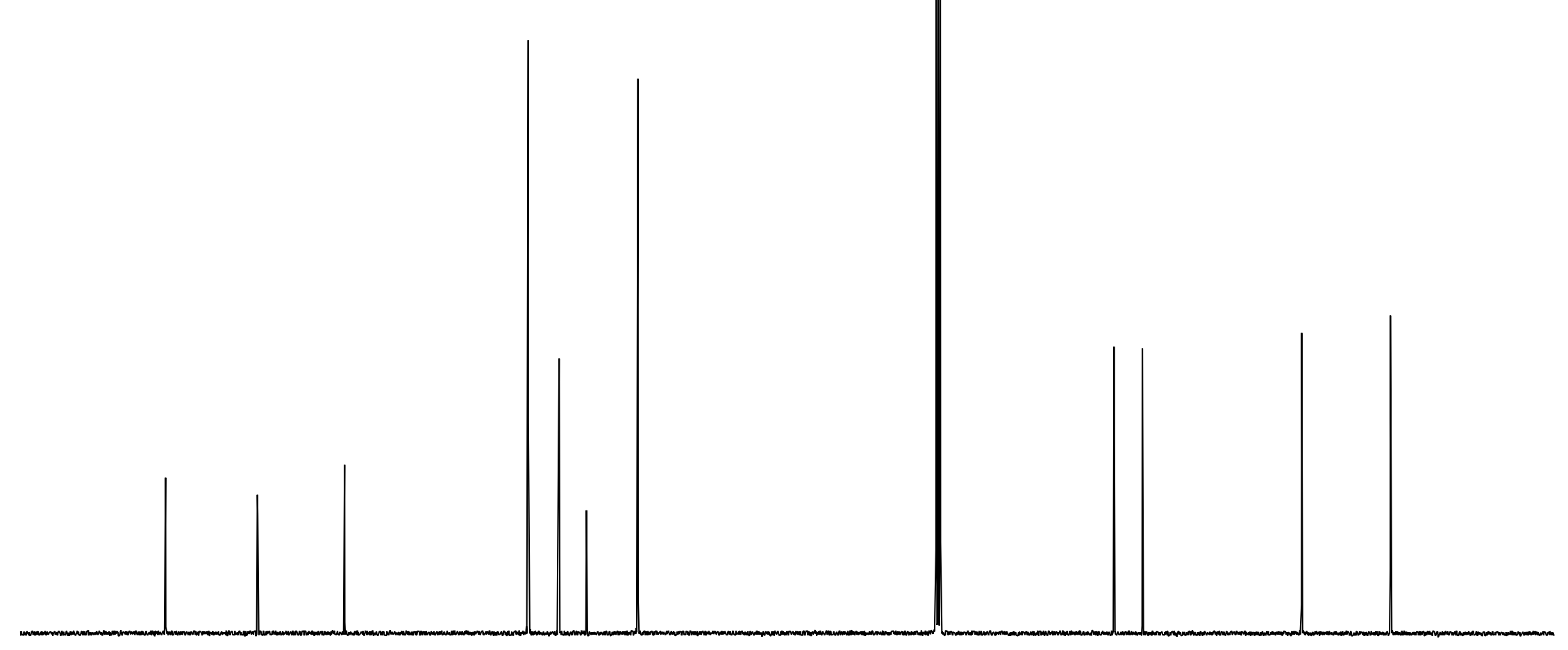

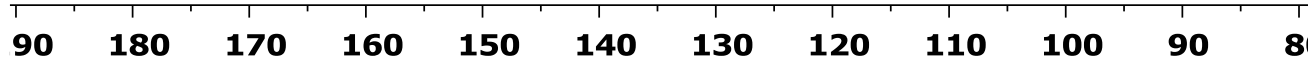

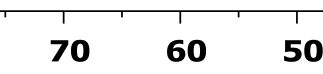

$40 \quad 30$

30 


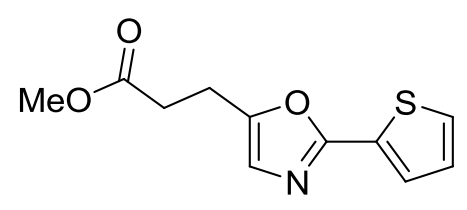

S2c
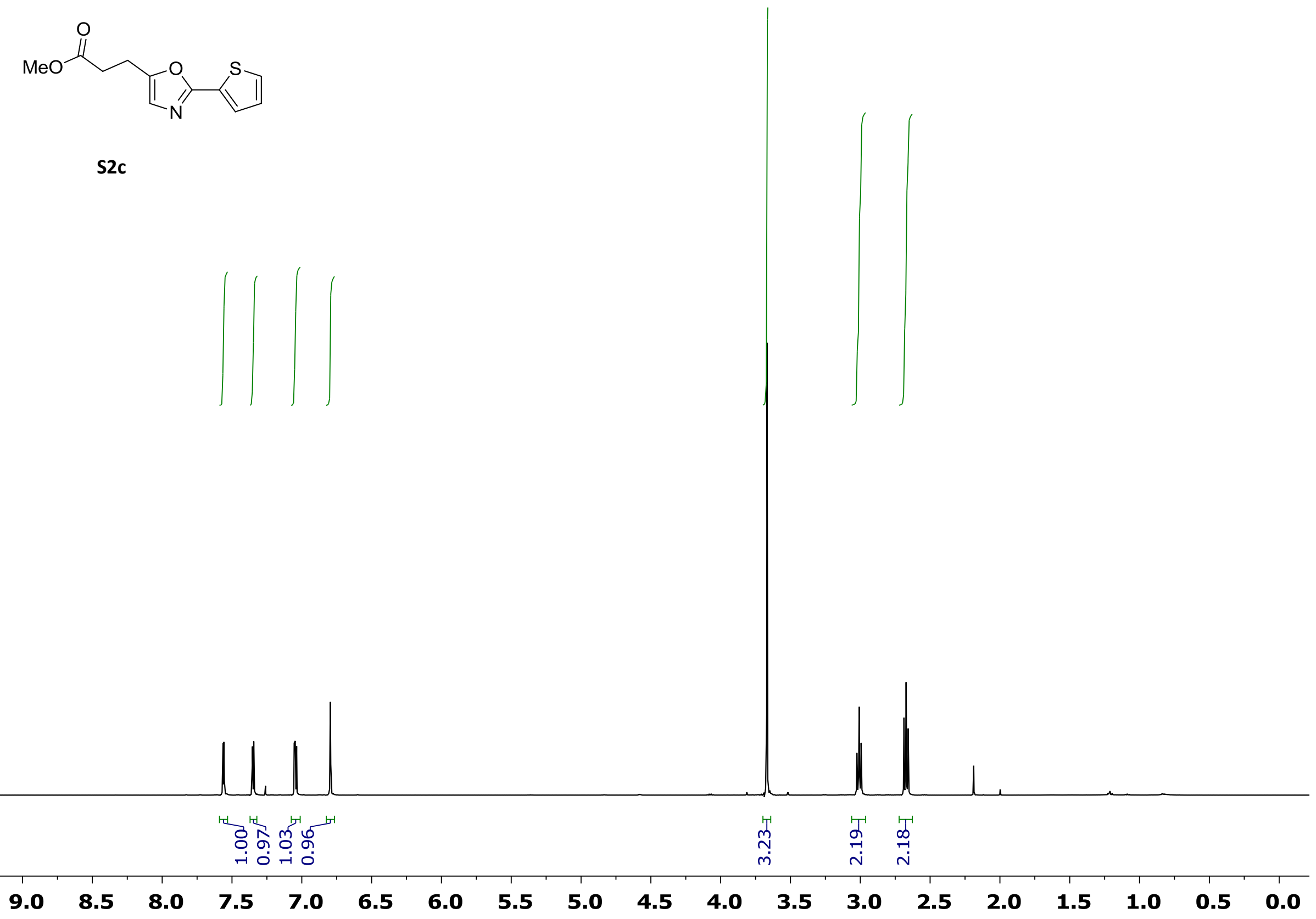


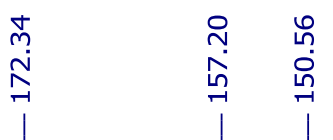

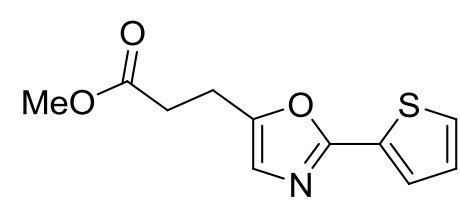

S2c

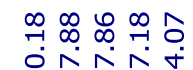

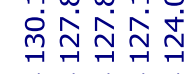

r

S2c

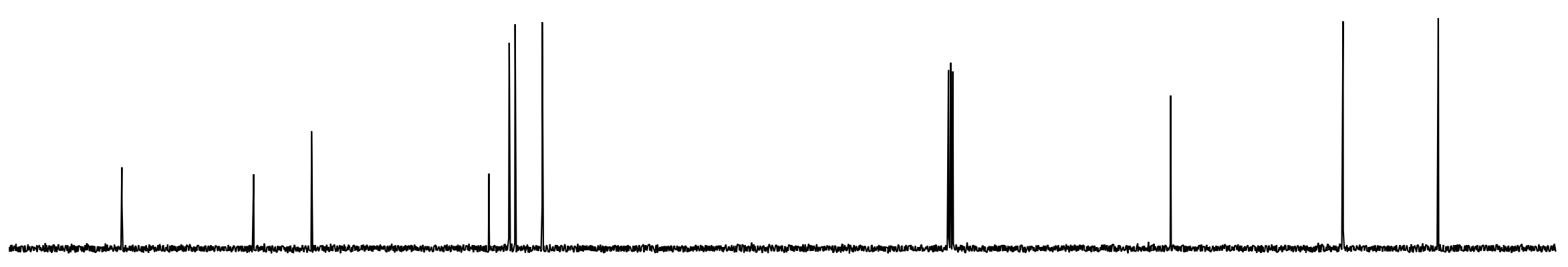




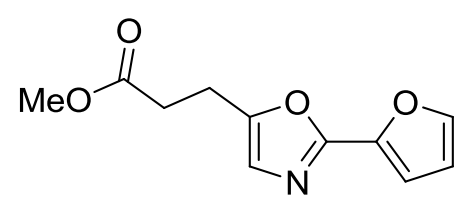

S2d

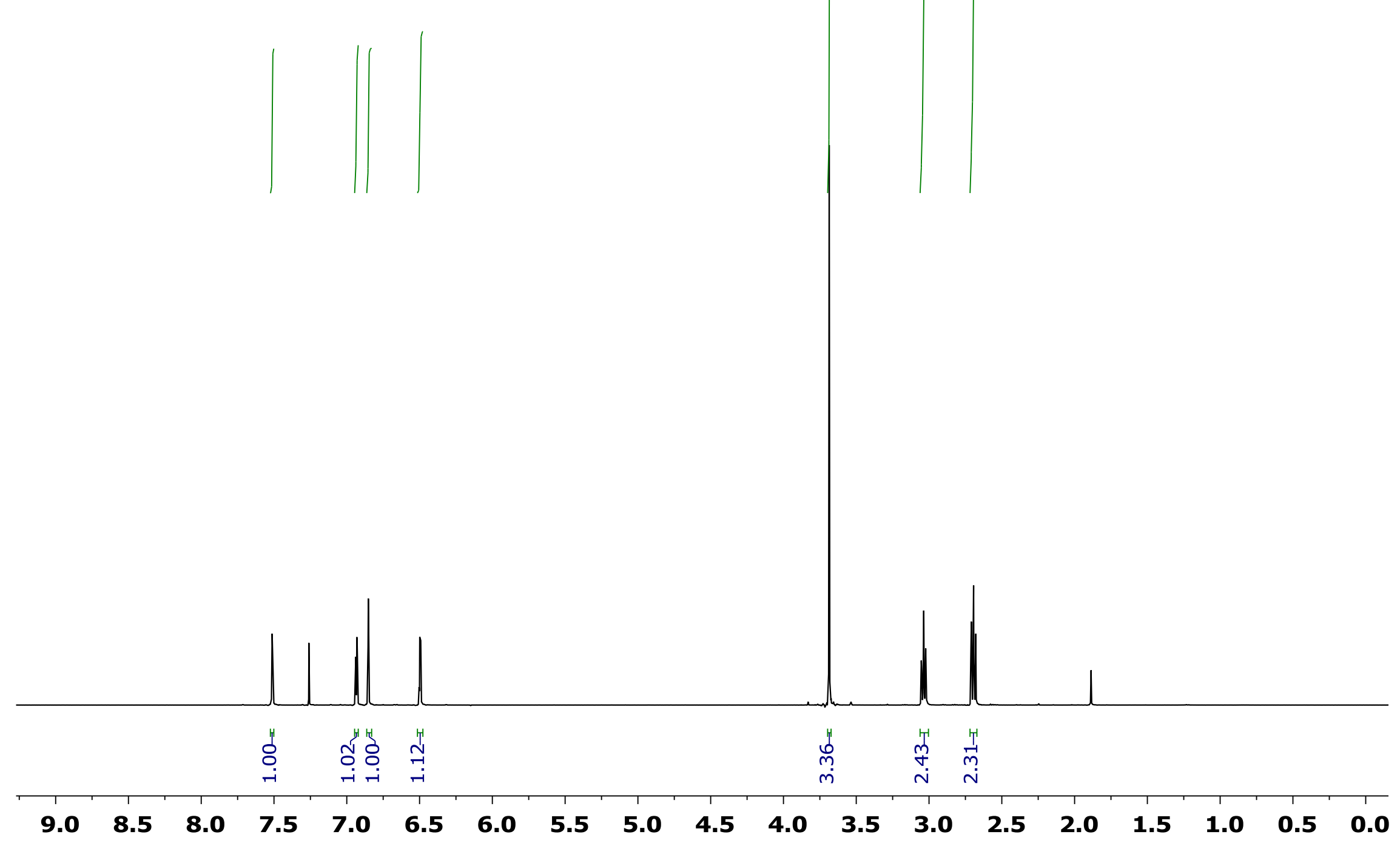




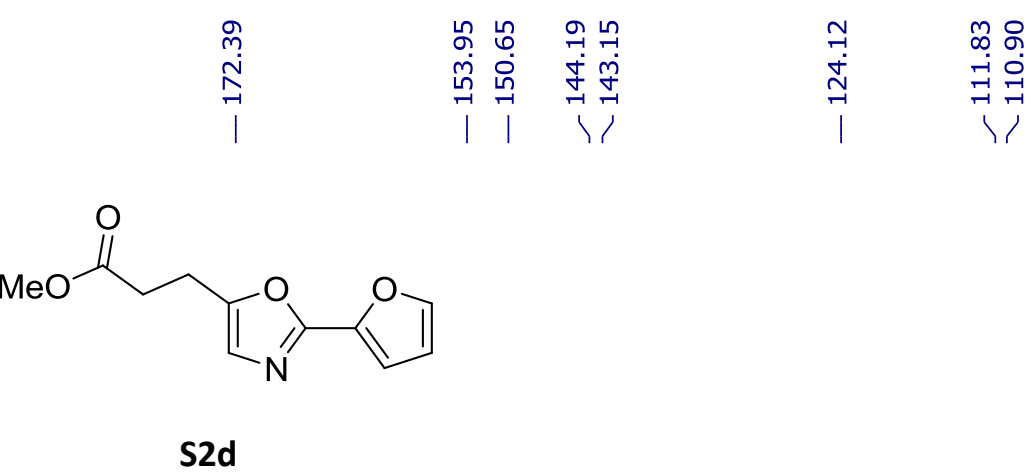

S2d
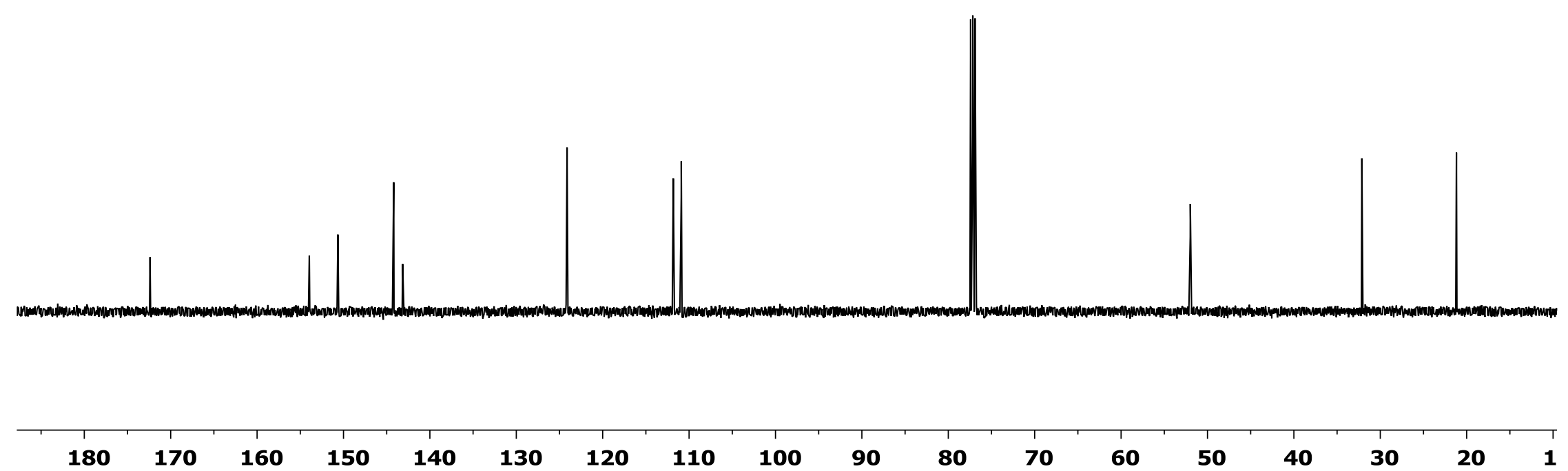


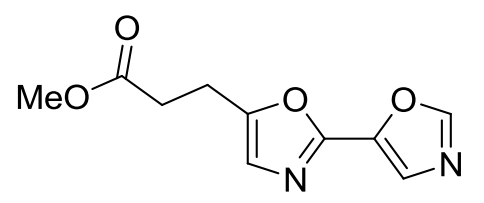

S2e

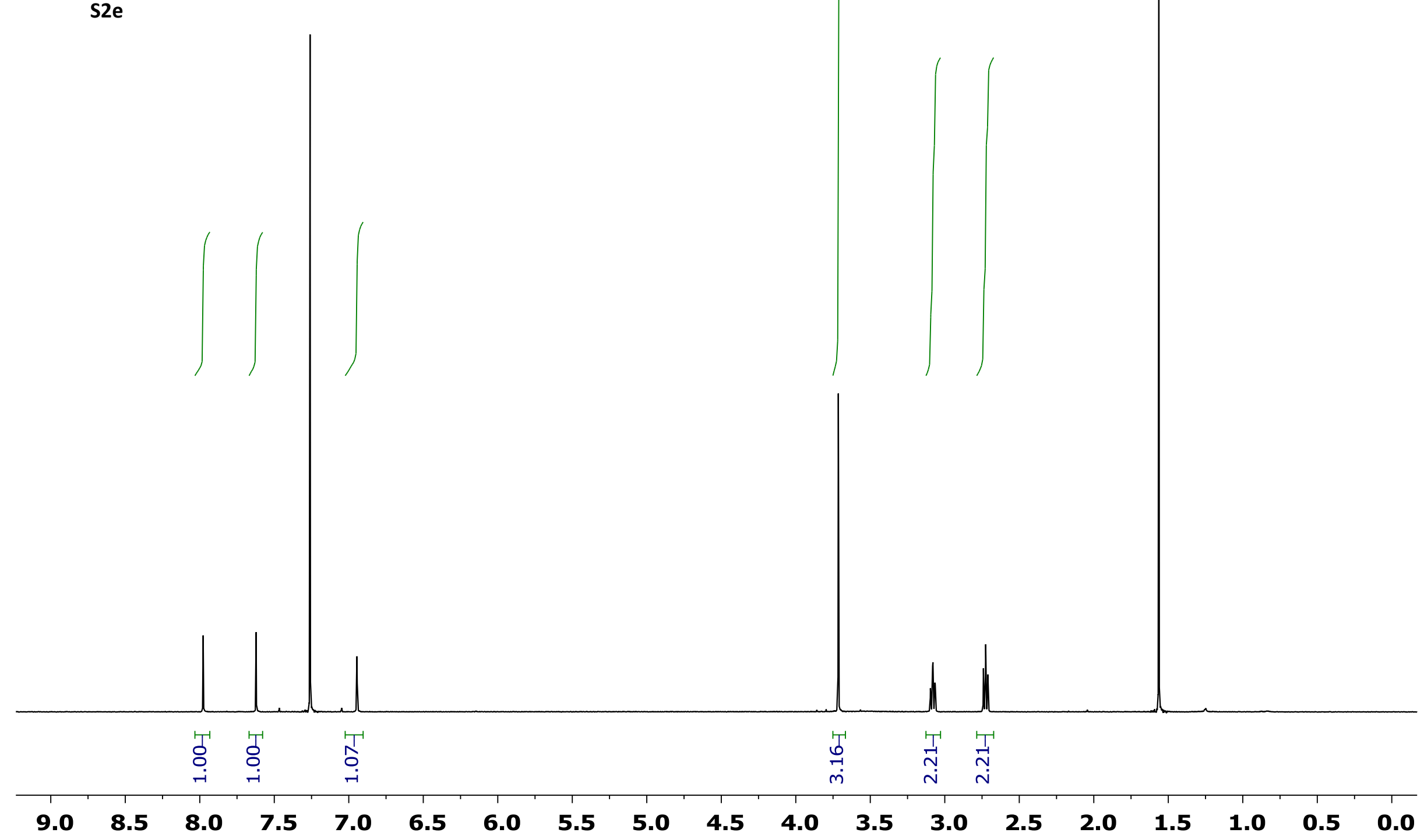




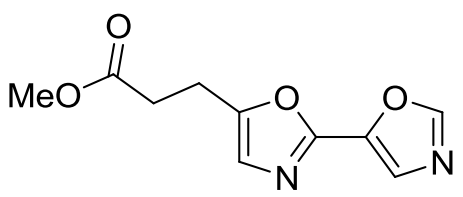

S2e

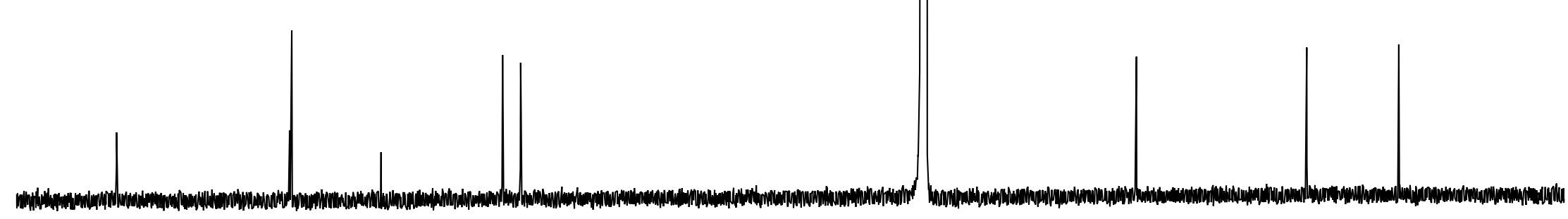

180

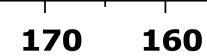

150

$140 \quad 130 \quad 120$

110100

90

80

70

60

50

40

30

20 
<smiles>O=C(O)CCc1cnc(-c2ccccc2)o1</smiles>

$1 a$

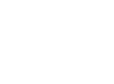




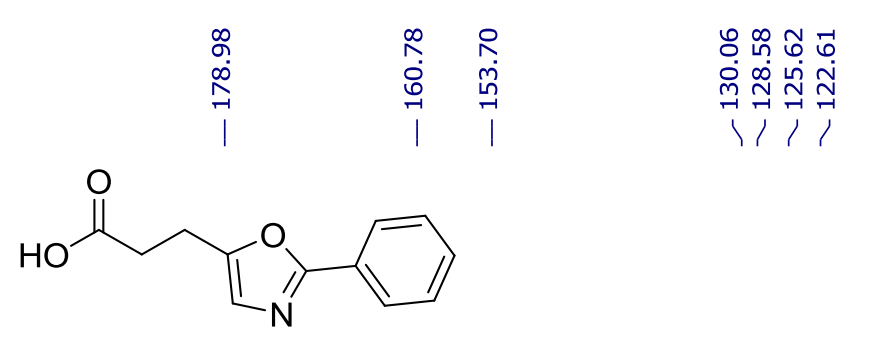

$1 a$

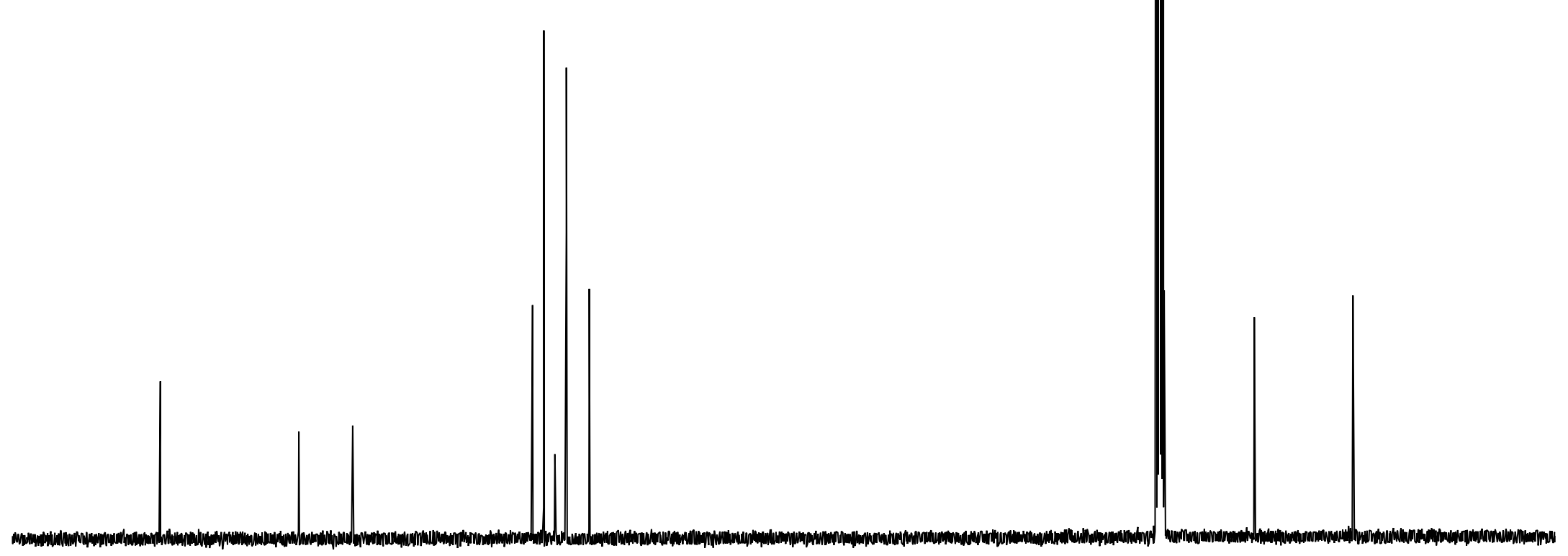

190180

$\begin{array}{llll}170 & 160 & 150 & 140\end{array}$

130

120

110100

90


<smiles>COc1ccc(-c2ncc(CCC(=O)O)o2)cc1</smiles>

$1 \mathrm{~b}$

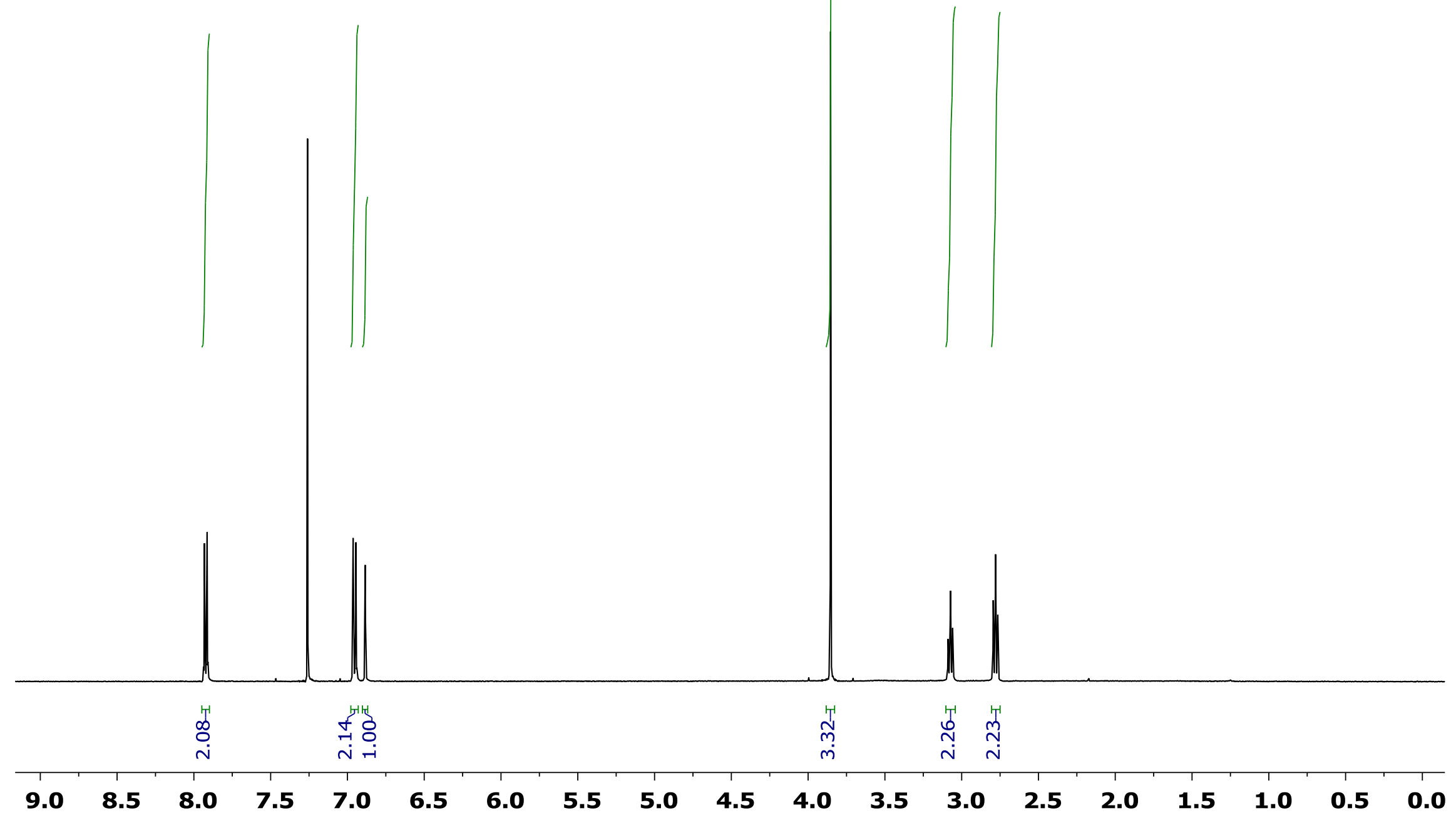




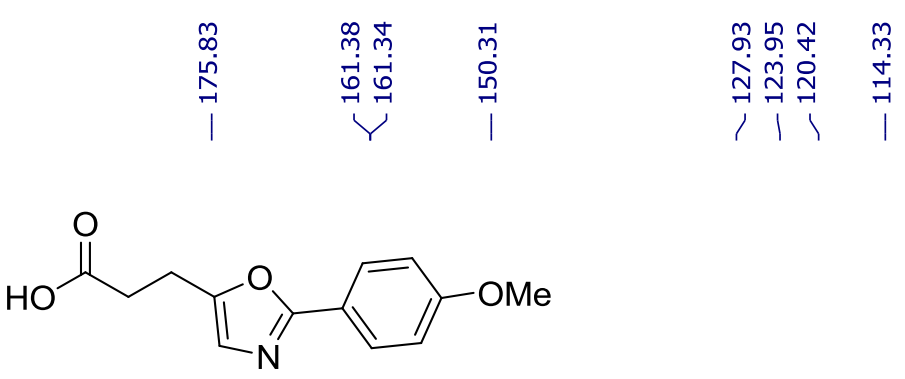

1b

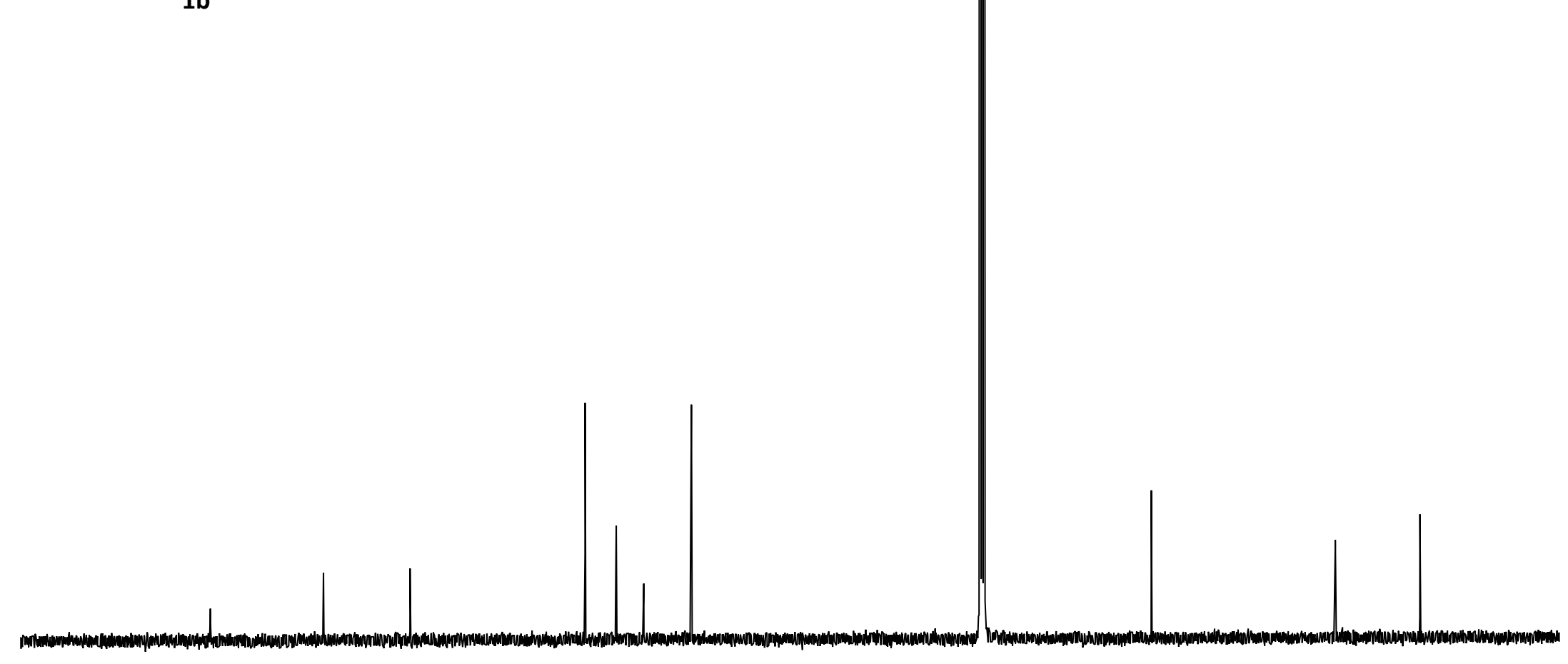

\begin{tabular}{lllllllllllllllllllll}
\hline 0 & 190 & 180 & 170 & 160 & 150 & 140 & 130 & 120 & 110 & 100 & 90 & 80 & 70 & 60 & 50 & 40 & 30 & 20 & 10 & 40
\end{tabular} 


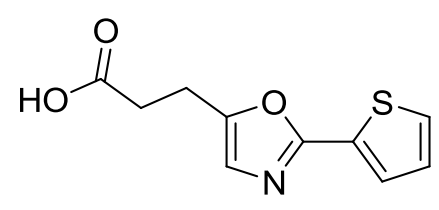

$1 c$

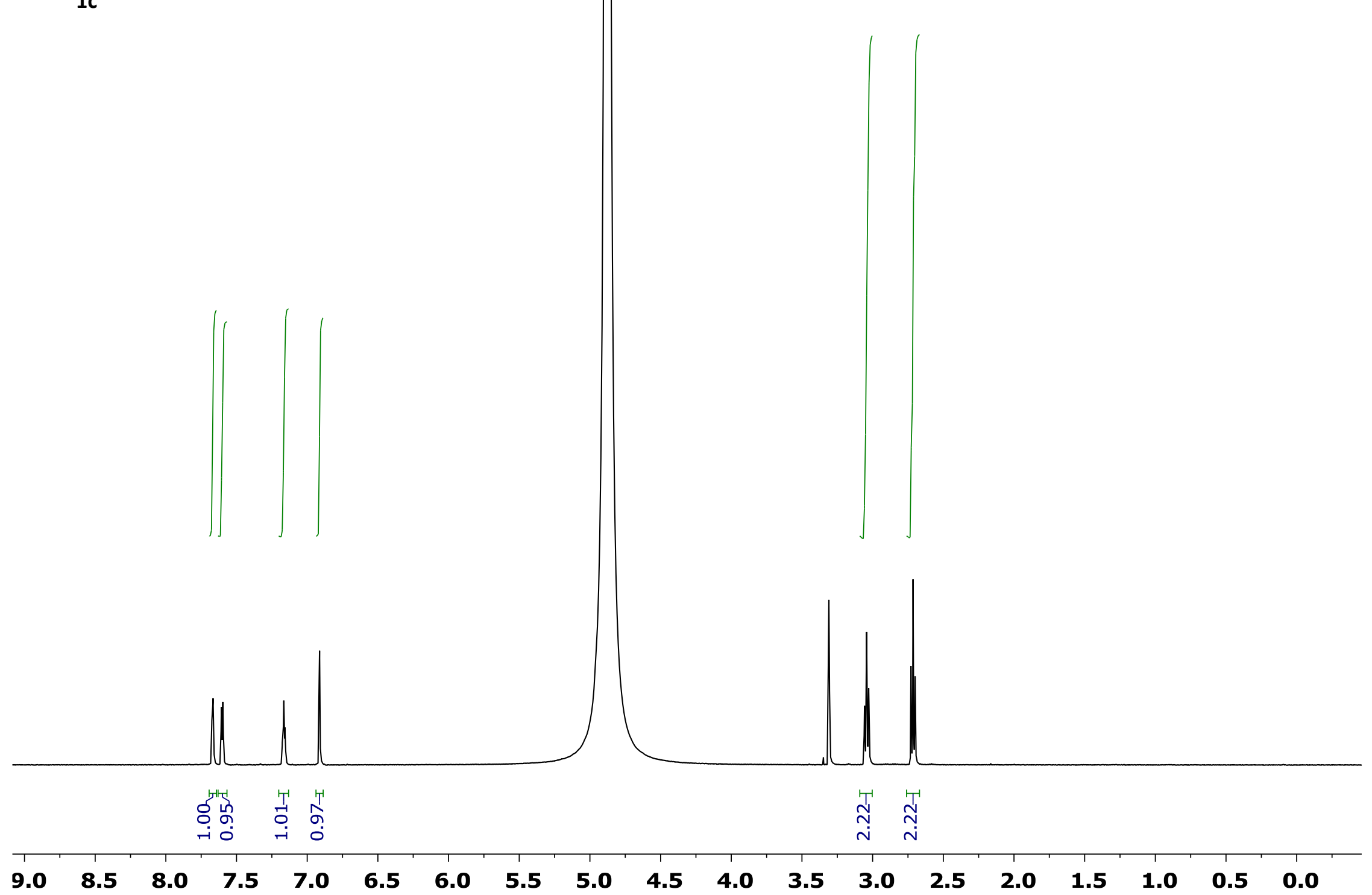




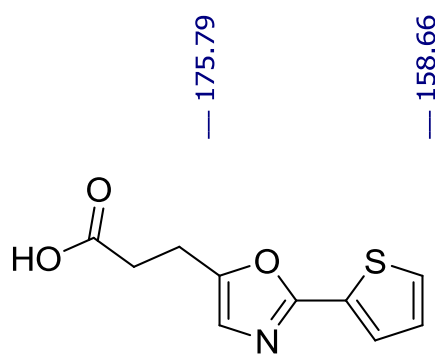

$\begin{array}{llll}0 & 7 \\ \infty & 0\end{array}$

1c

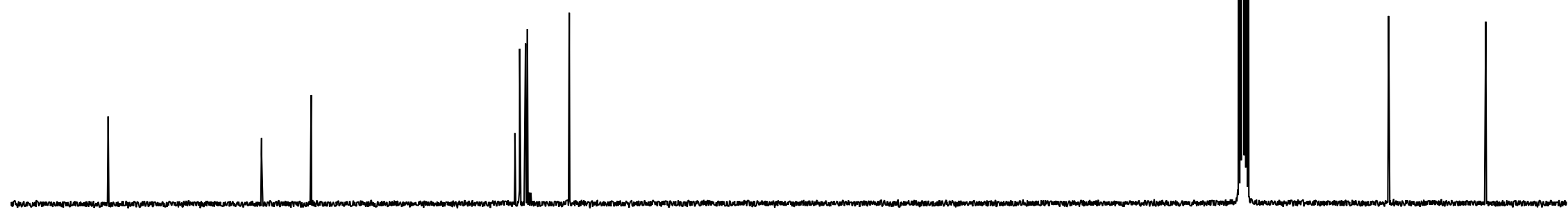

180

170160

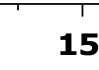

150140

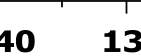


Photoassisted Diversity-Oriented Synthesis: Intramolecular Cycloadditions...

N.N. Bhuvan Kumar and Andrei G. Kutateladze, S55

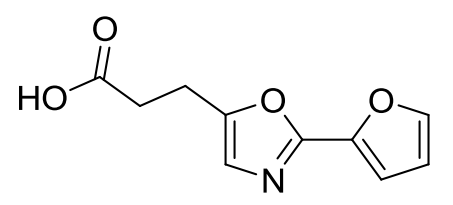

$1 d$

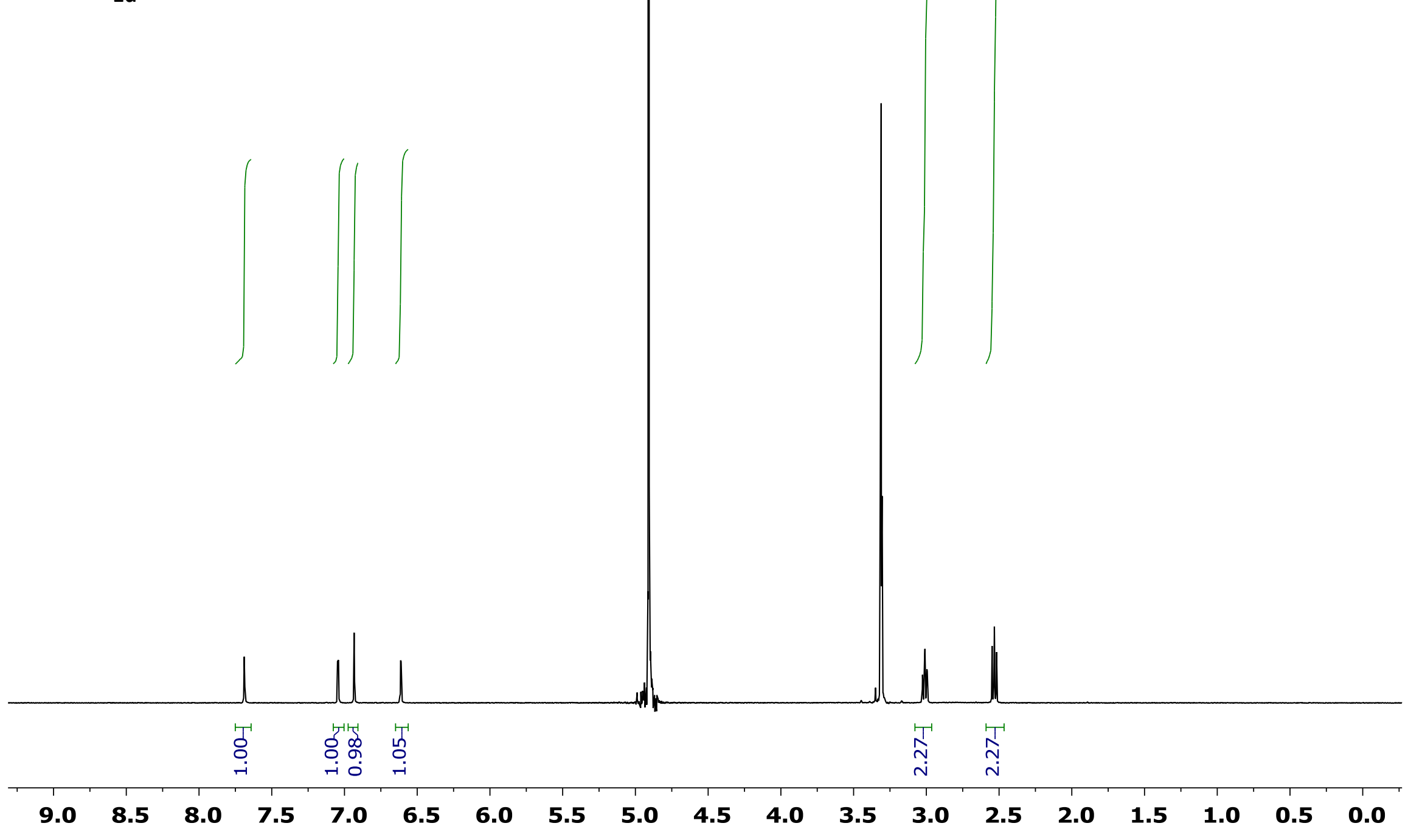



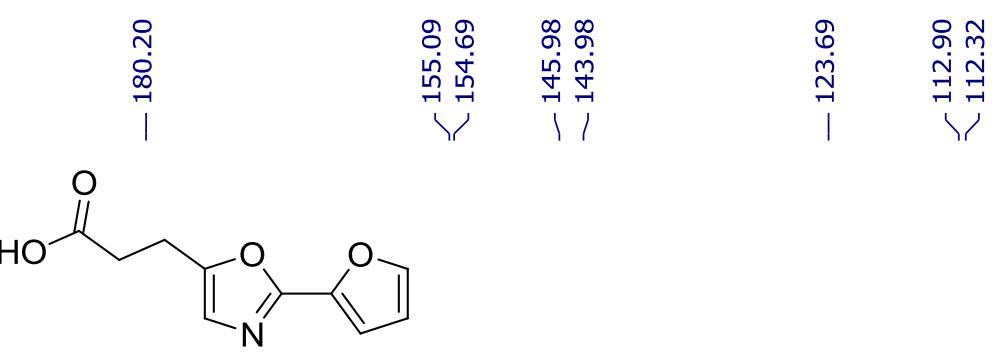

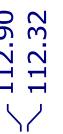

$1 d$

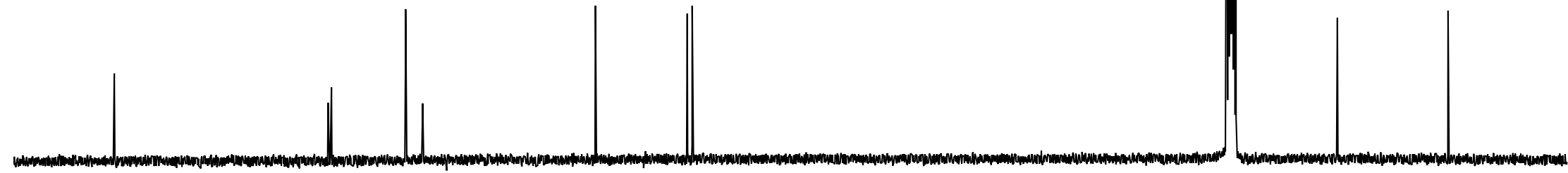

190 180 170 160 150 140130 120 
<smiles>O=C(O)CCc1cnc(-c2cnco2)o1</smiles>

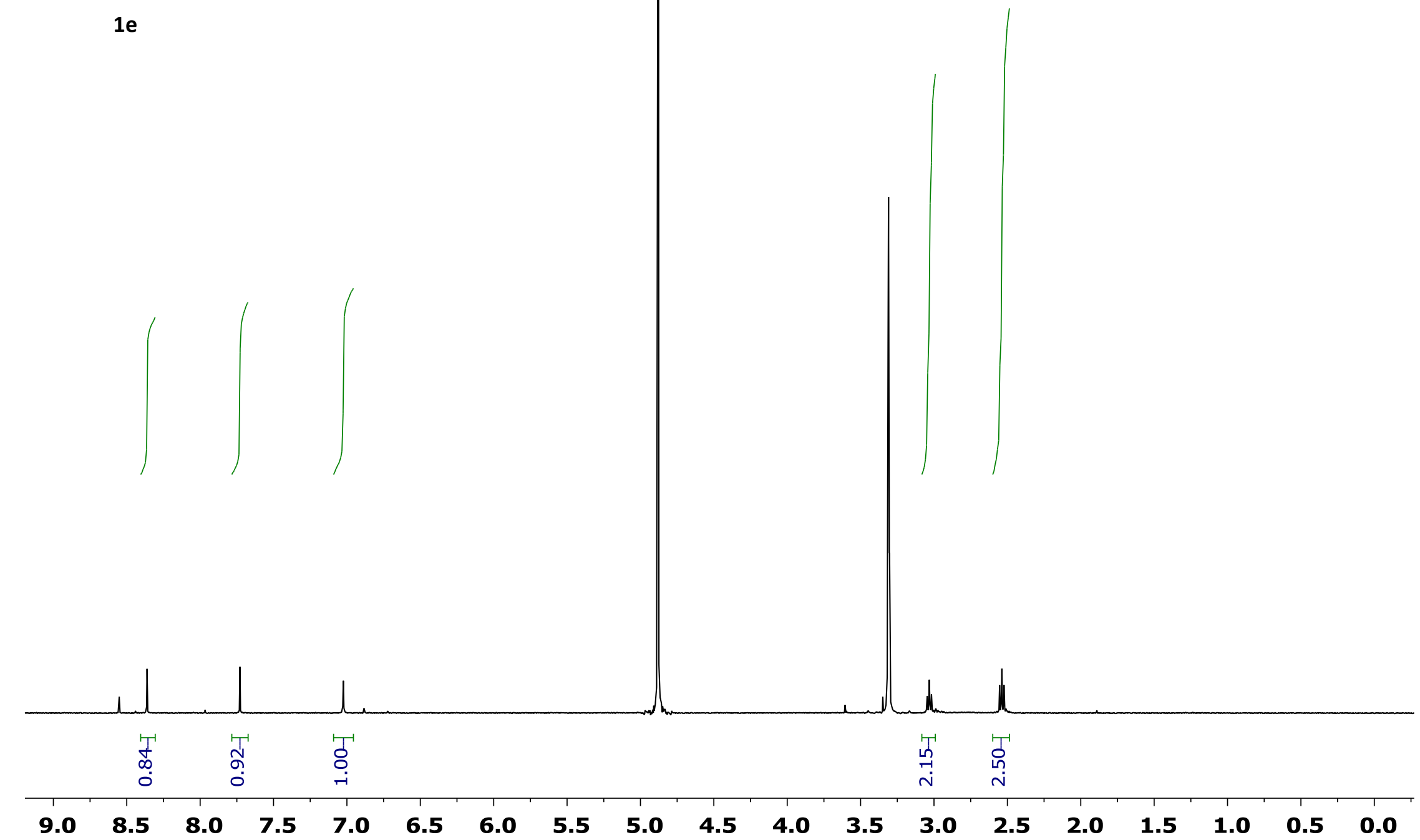




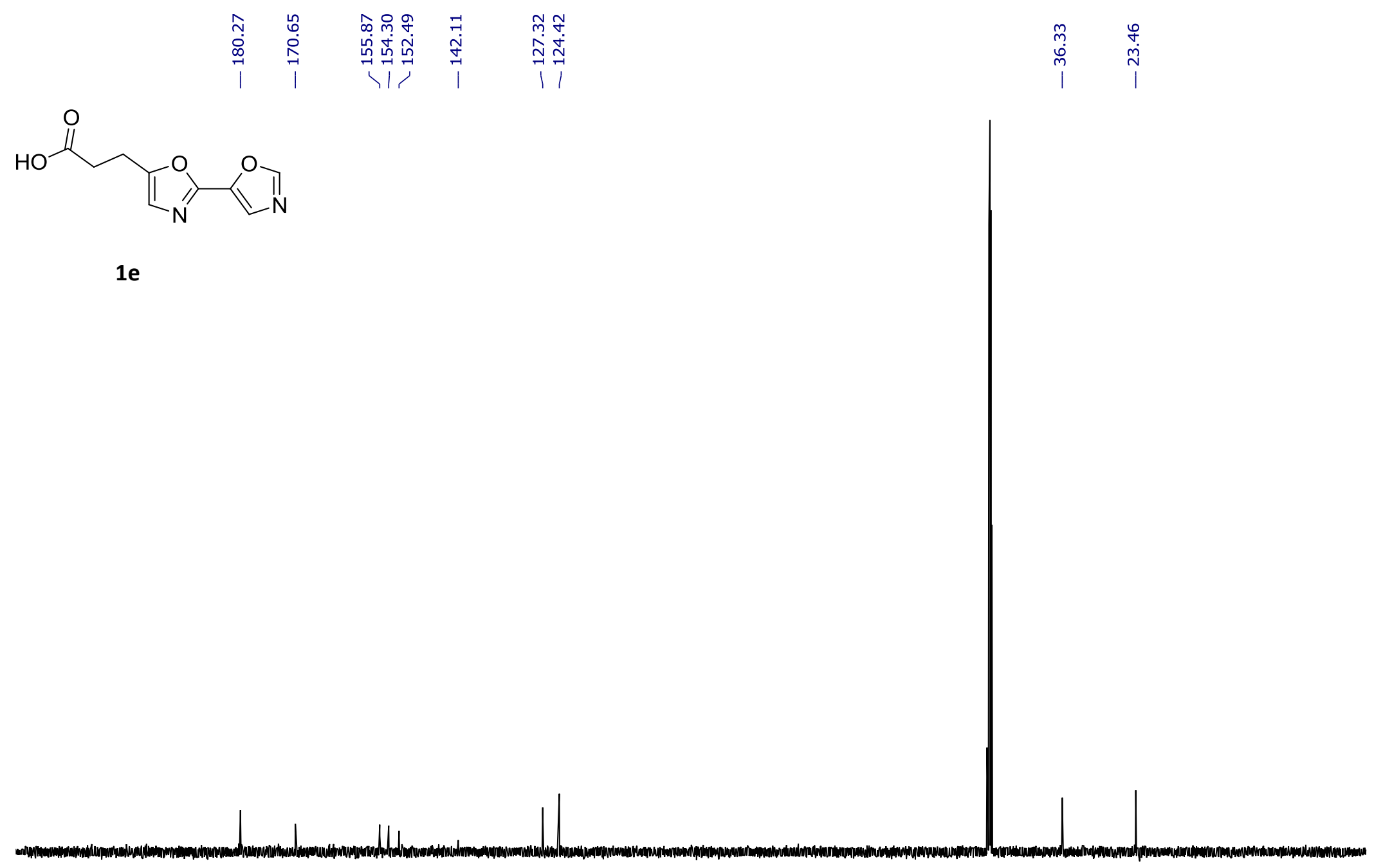

$\begin{array}{lllllllllllllllllllllll}210 & 200 & 190 & 180 & 170 & 160 & 150 & 140 & 130 & 120 & 110 & 100 & 90 & 80 & 70 & 60 & 50 & 40 & 30 & 20 & 10 & 0 & -10\end{array}$ 


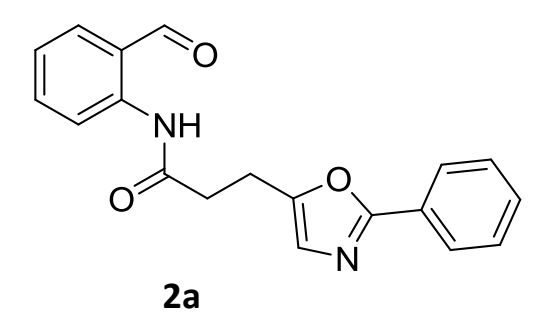

$2 a$
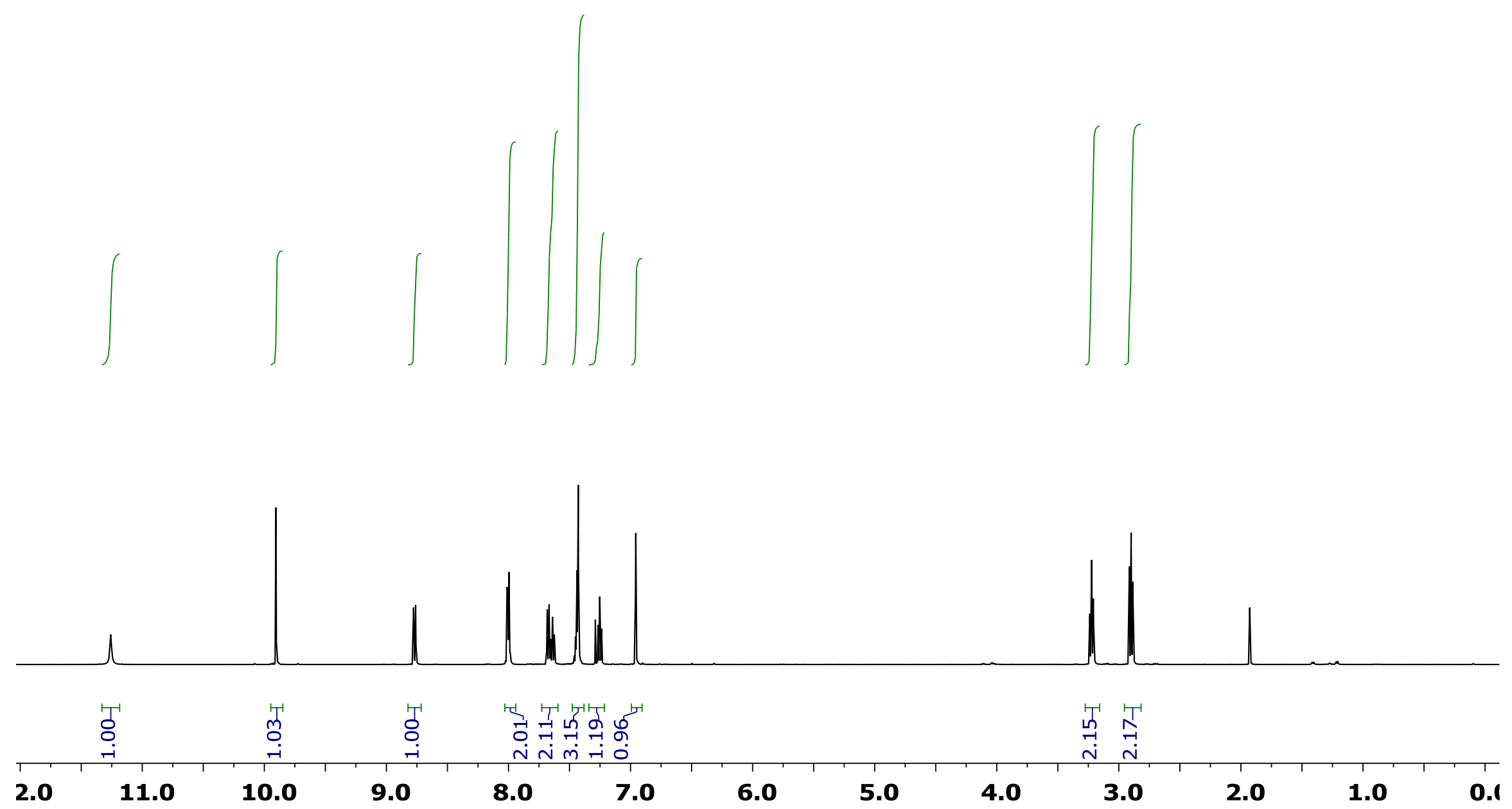


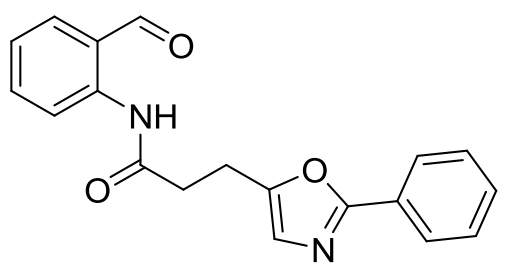

$2 a$

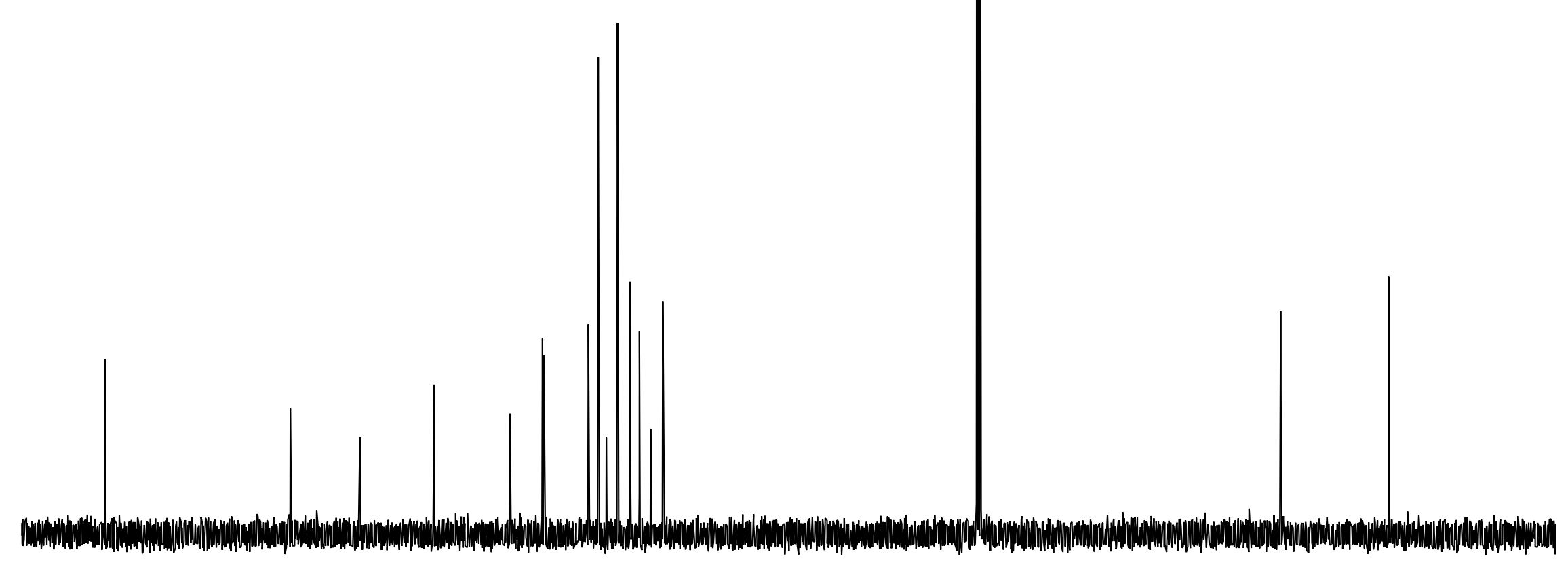




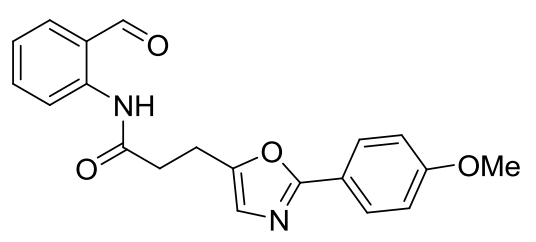

2b
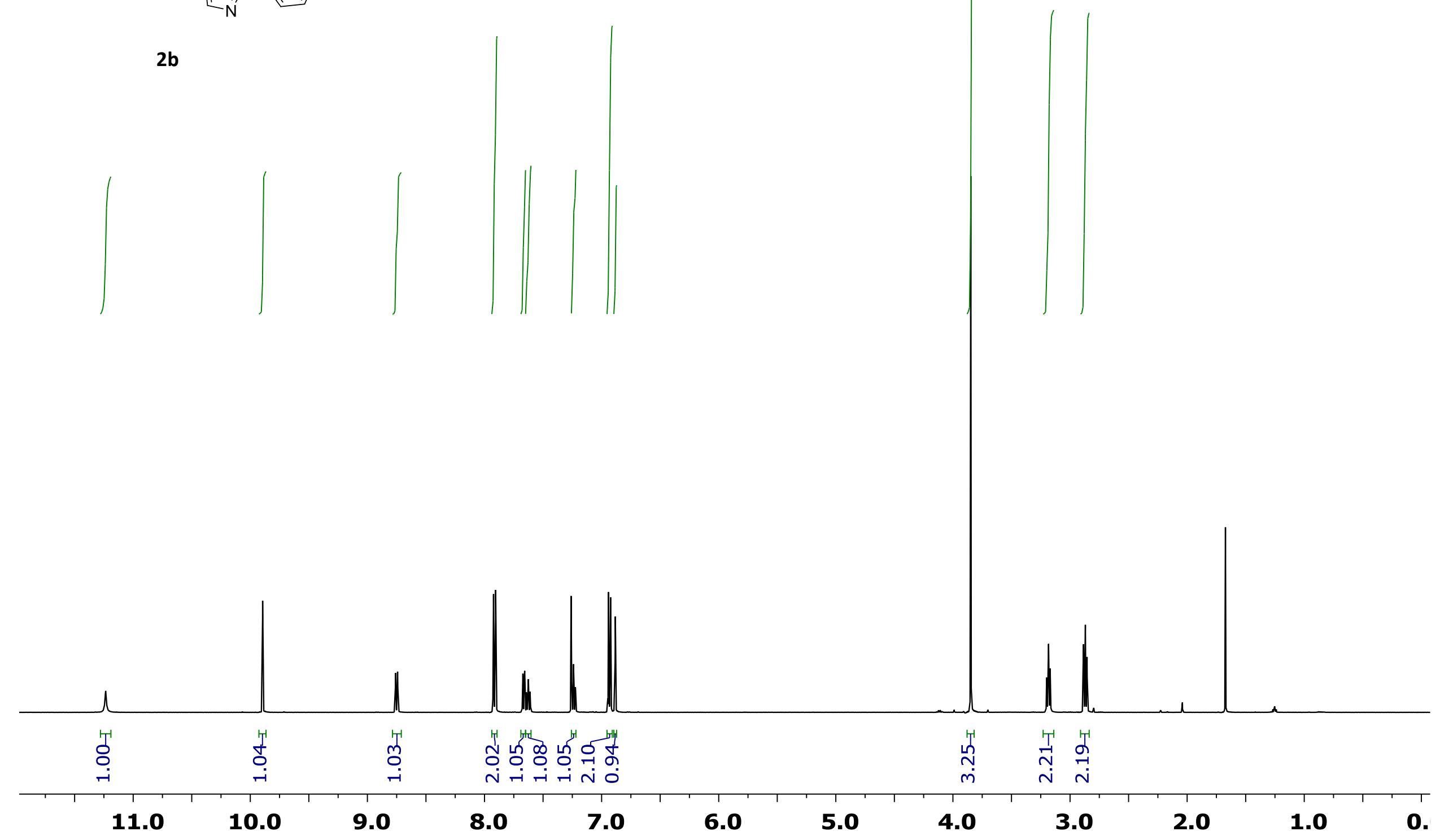


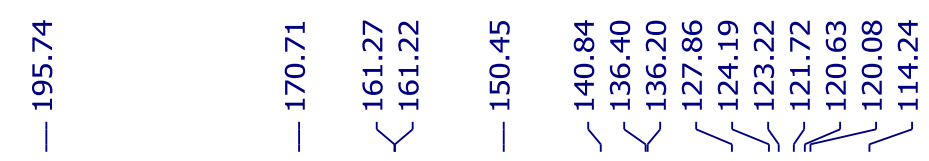

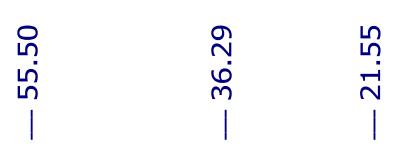

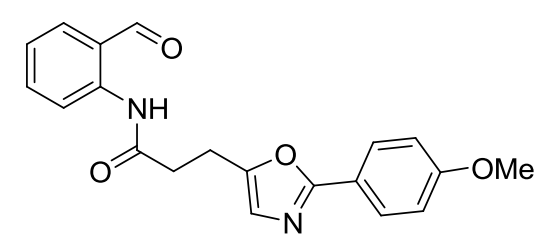

$2 b$

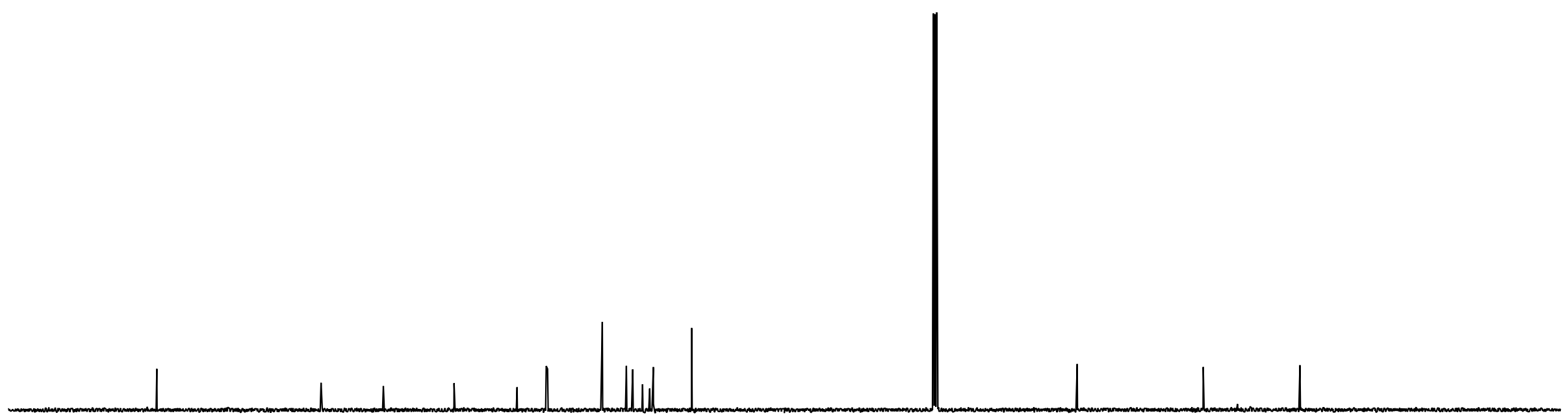

$\begin{array}{lllllllllllllllllllllll}210 & 200 & 190 & 180 & 170 & 160 & 150 & 140 & 130 & 120 & 110 & 100 & 90 & 80 & 70 & 60 & 50 & 40 & 30 & 20 & 10 & 0 & -10\end{array}$ 


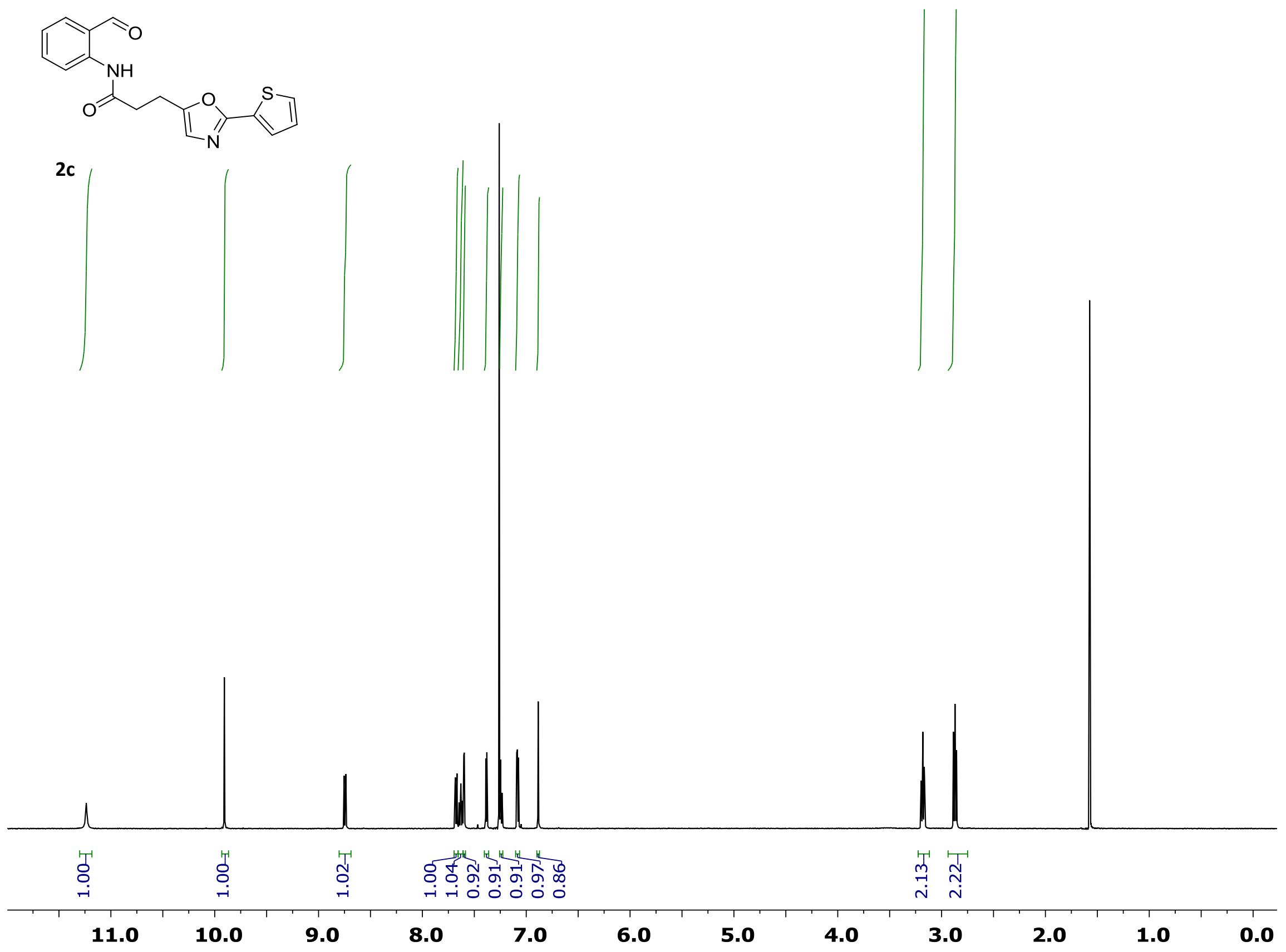


Photoassisted Diversity-Oriented Synthesis: Intramolecular Cycloadditions...

N.N. Bhuvan Kumar and Andrei G. Kutateladze, S64

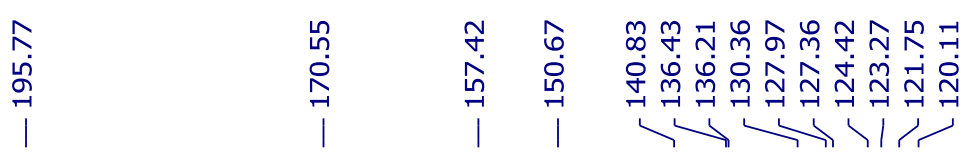

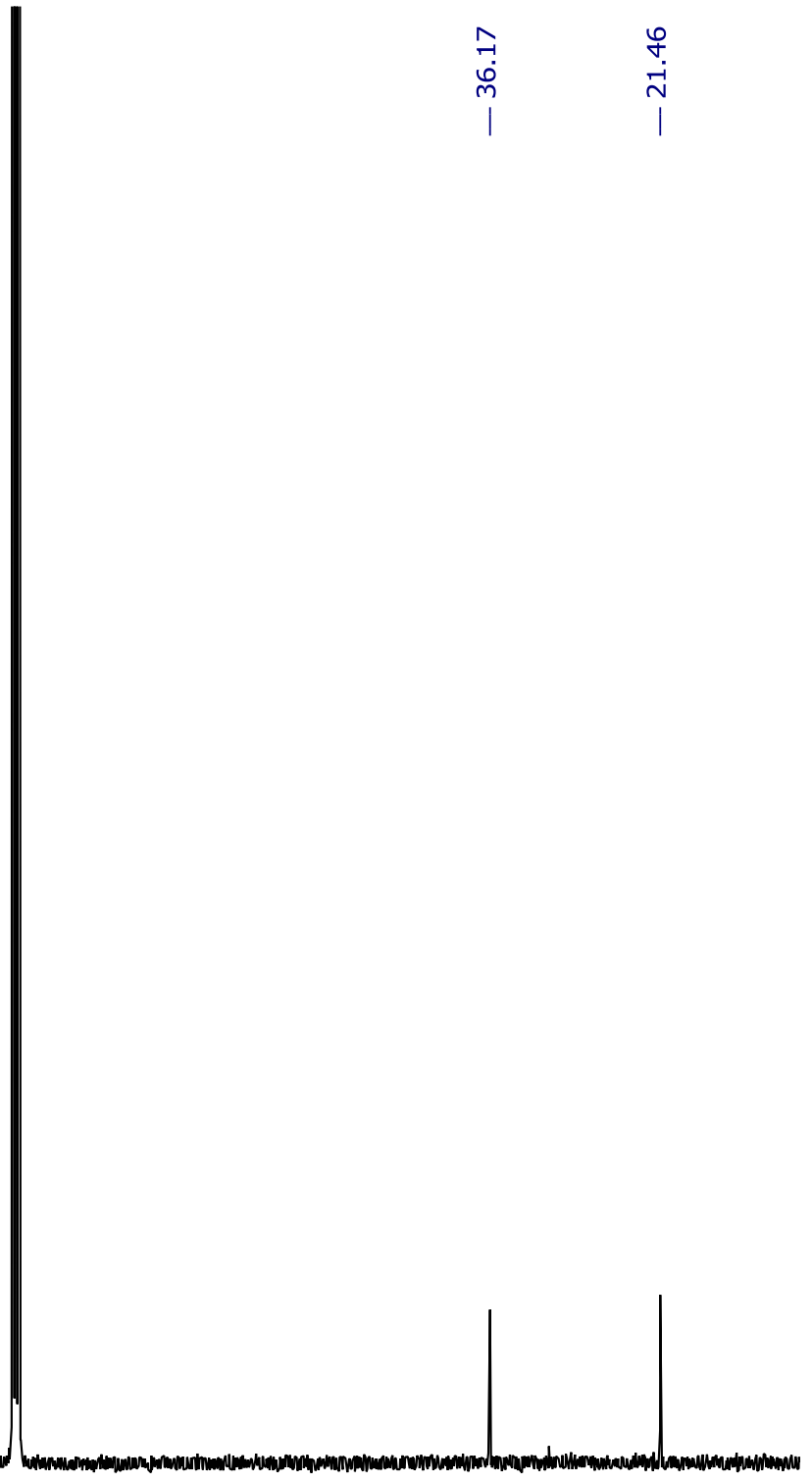<smiles>O=Cc1ccccc1NC(=O)CCc1cnc(-c2cccs2)o1</smiles>

2c
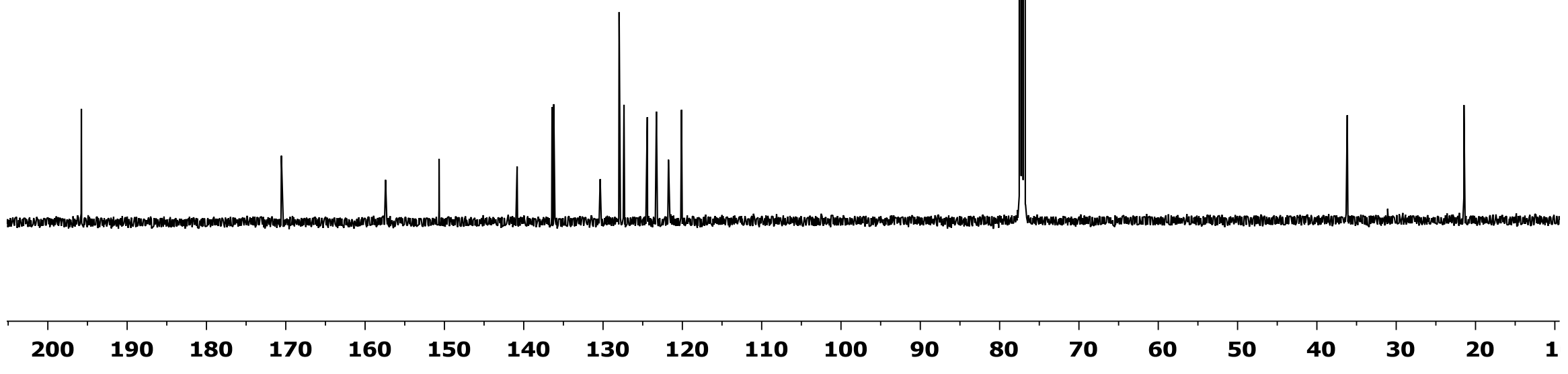
Photoassisted Diversity-Oriented Synthesis: Intramolecular Cycloadditions...

N.N. Bhuvan Kumar and Andrei G. Kutateladze, 565<smiles>O=Cc1ccccc1NC(=O)CCc1cnc(-c2ccco2)o1</smiles>

2d
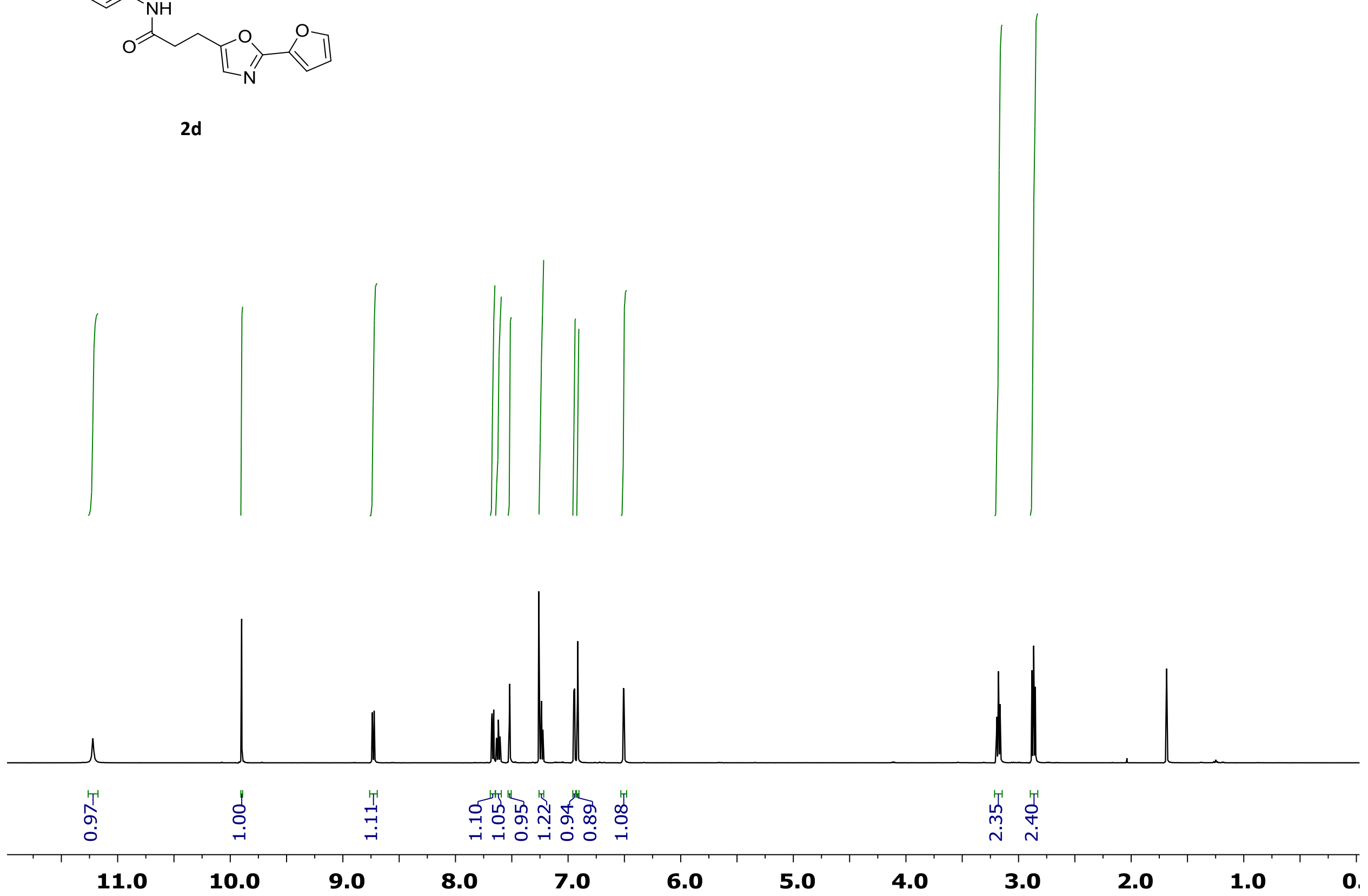


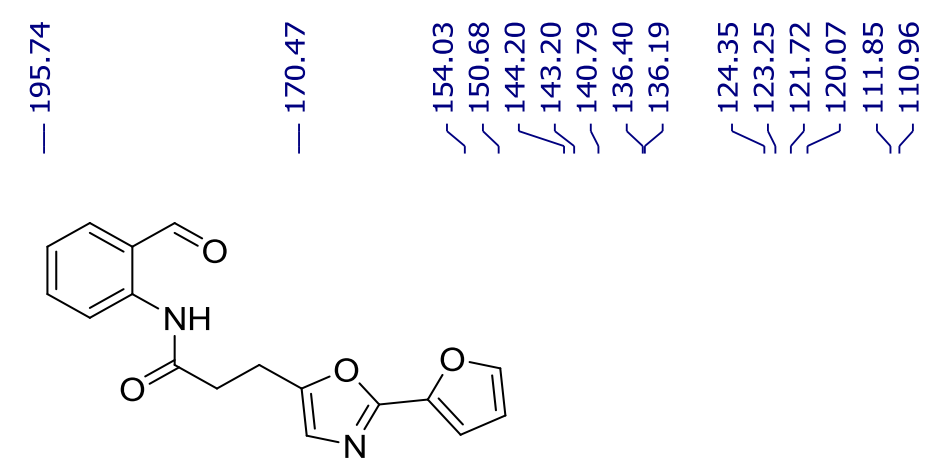

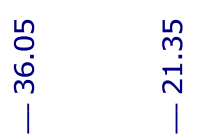

2d

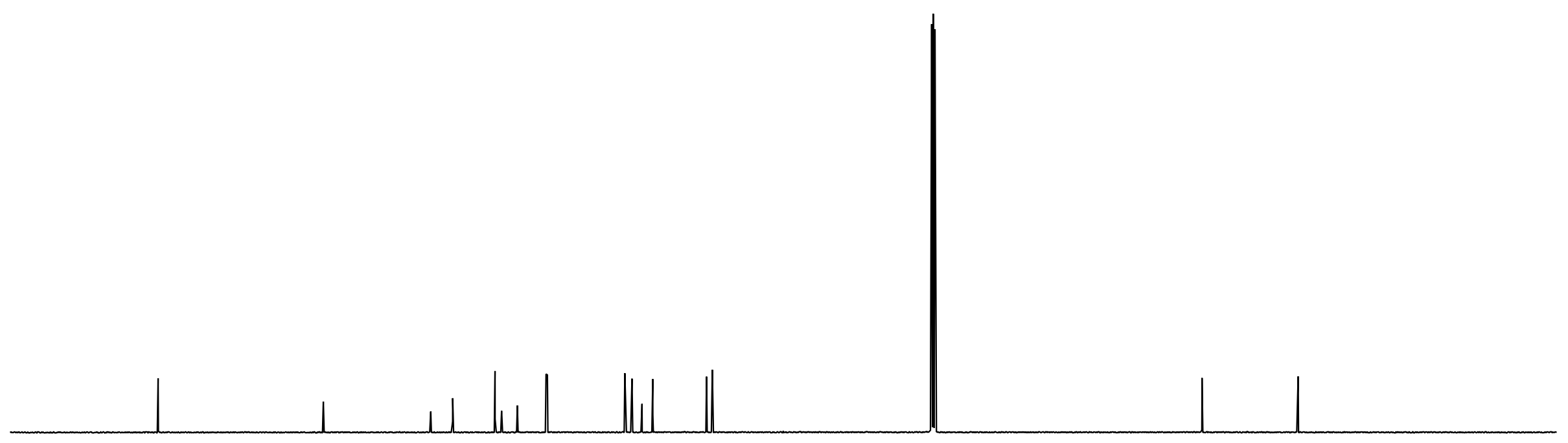

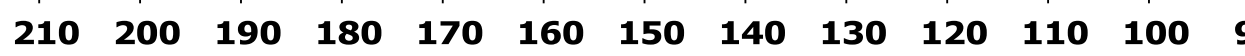

$90 \quad 80 \quad 70 \quad 60 \quad 50$

40

20

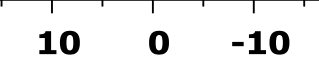




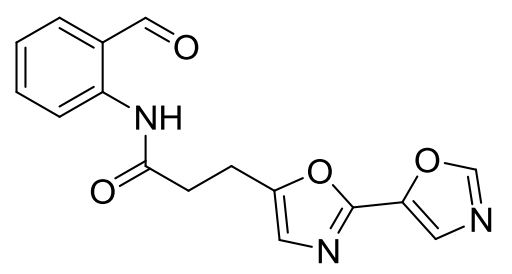

$2 e$

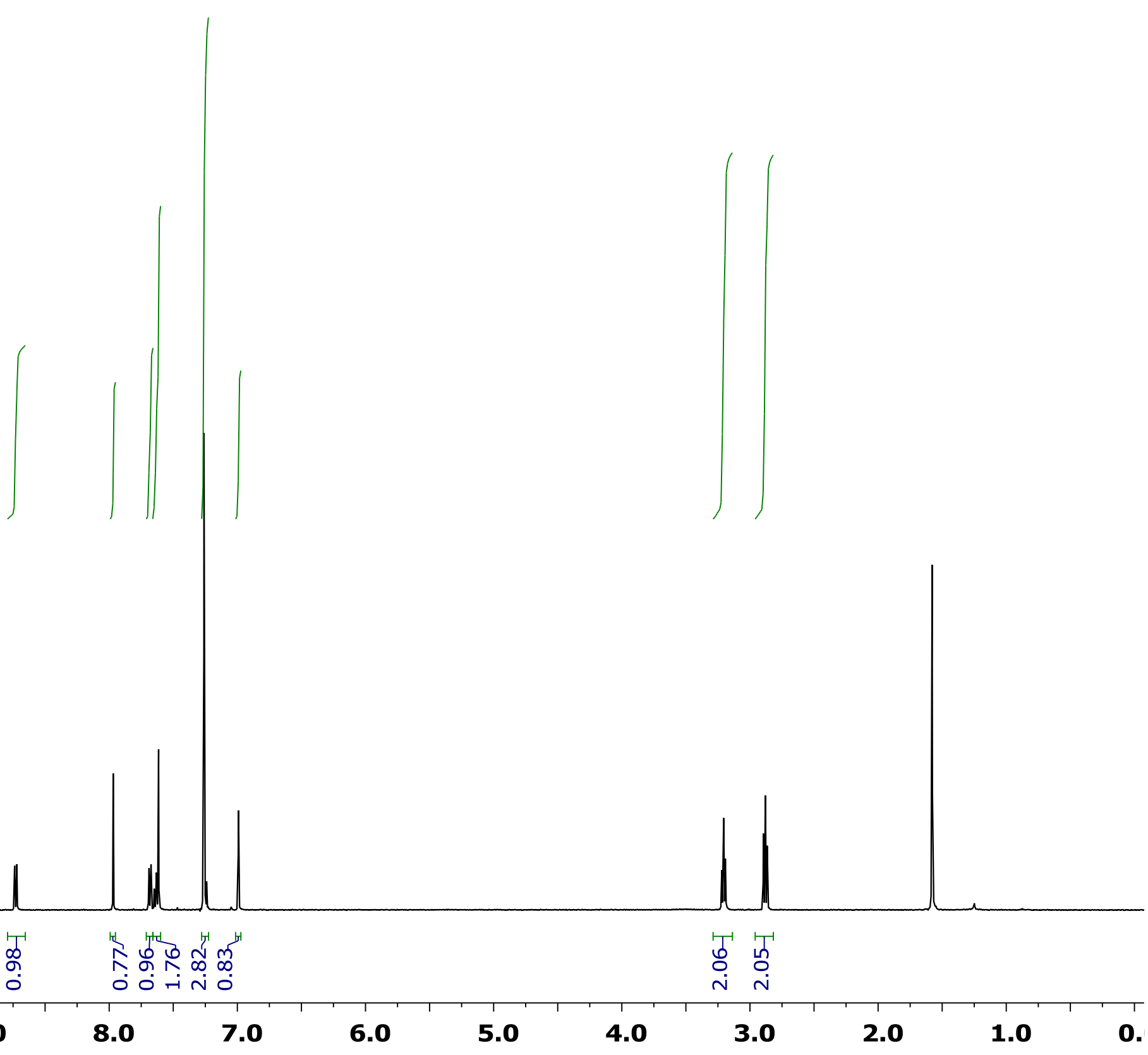



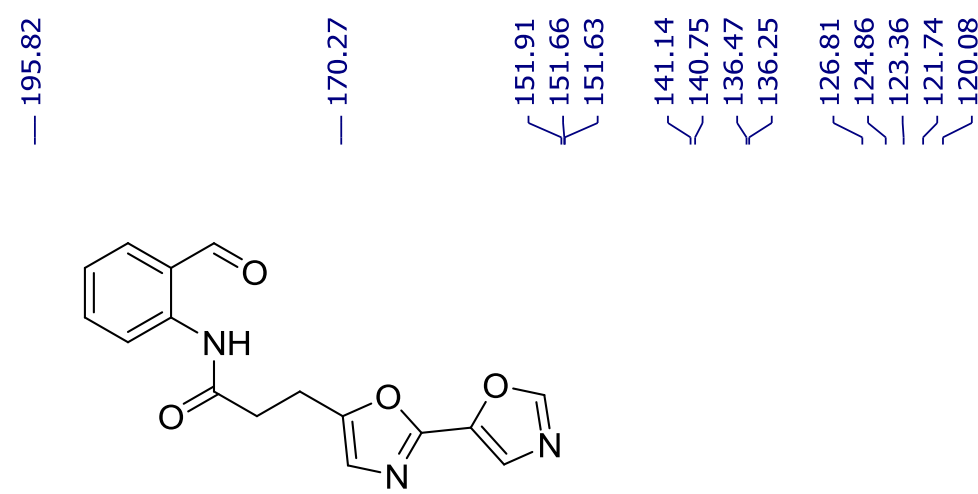

$2 \mathrm{e}$

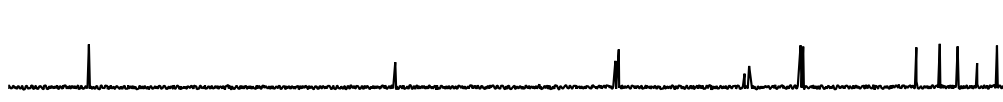




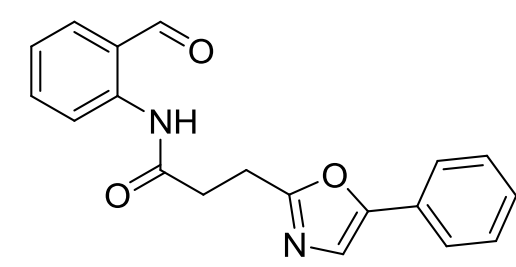

9f
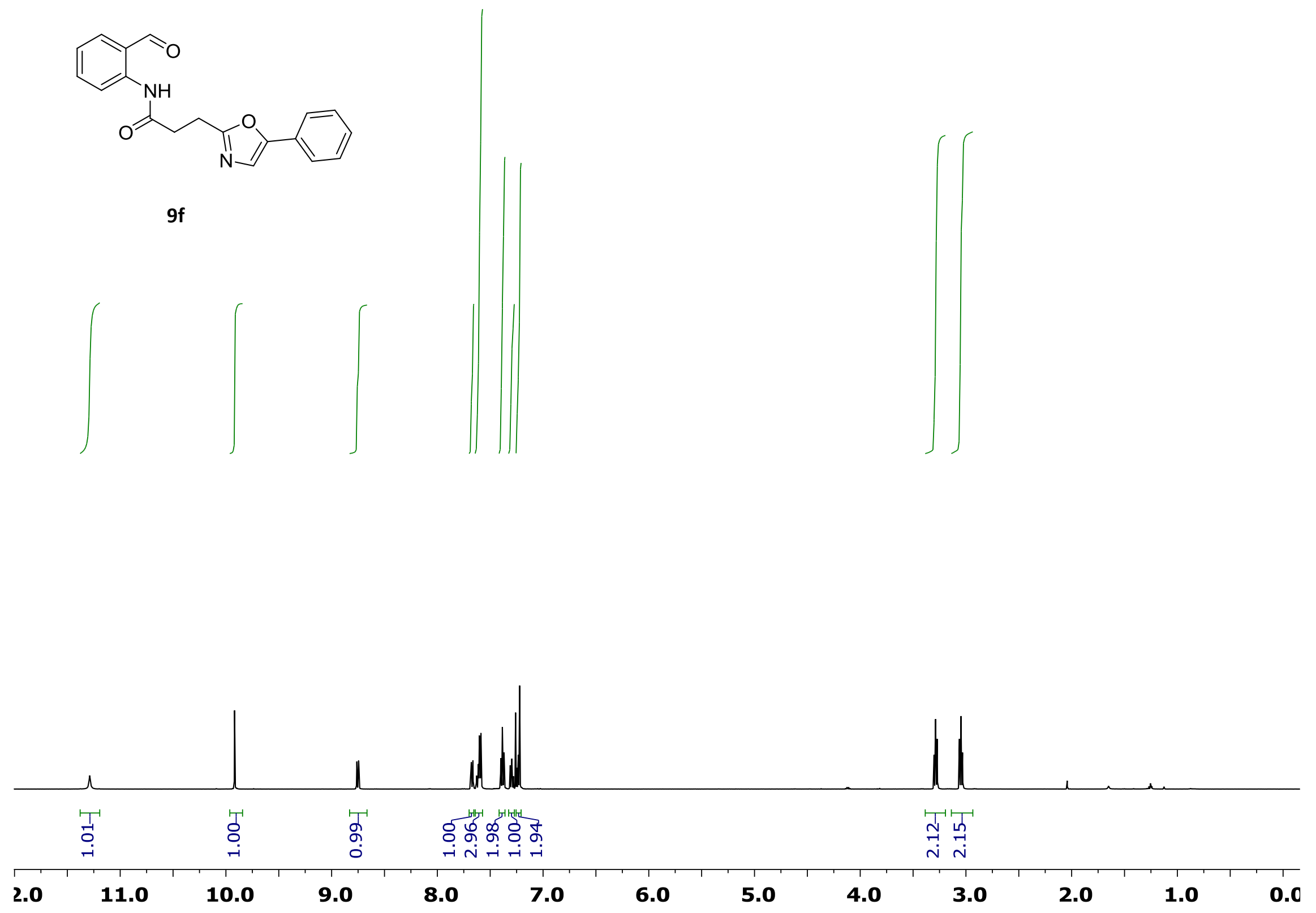


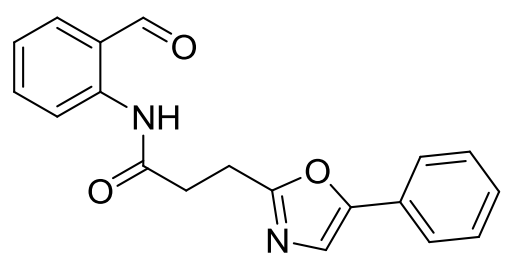

9f

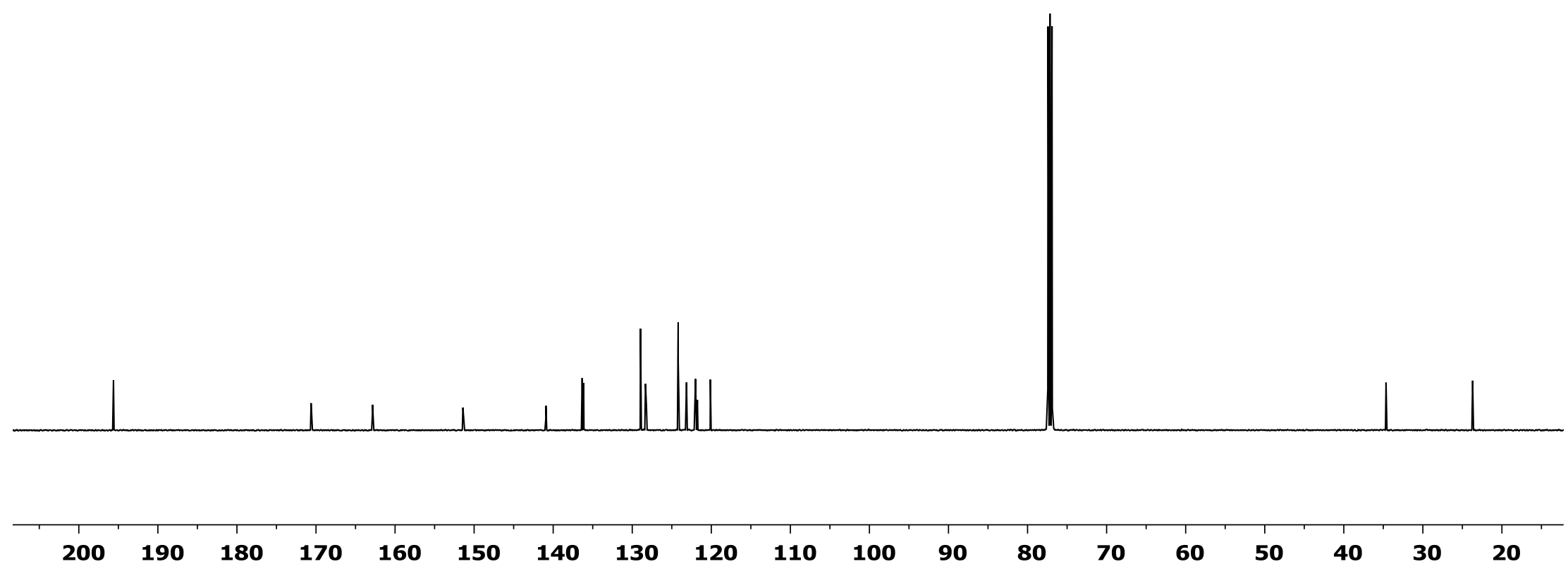

$\begin{array}{lllllllllllllllllll}200 & 190 & 180 & 170 & 160 & 150 & 140 & 130 & 120 & 110 & 100 & 90 & 80 & 70 & 60 & 50 & 40 & 30 & 20\end{array}$ 


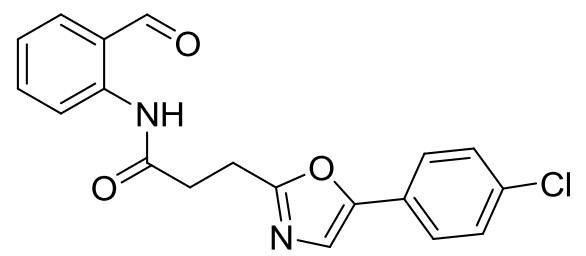

$9 g$
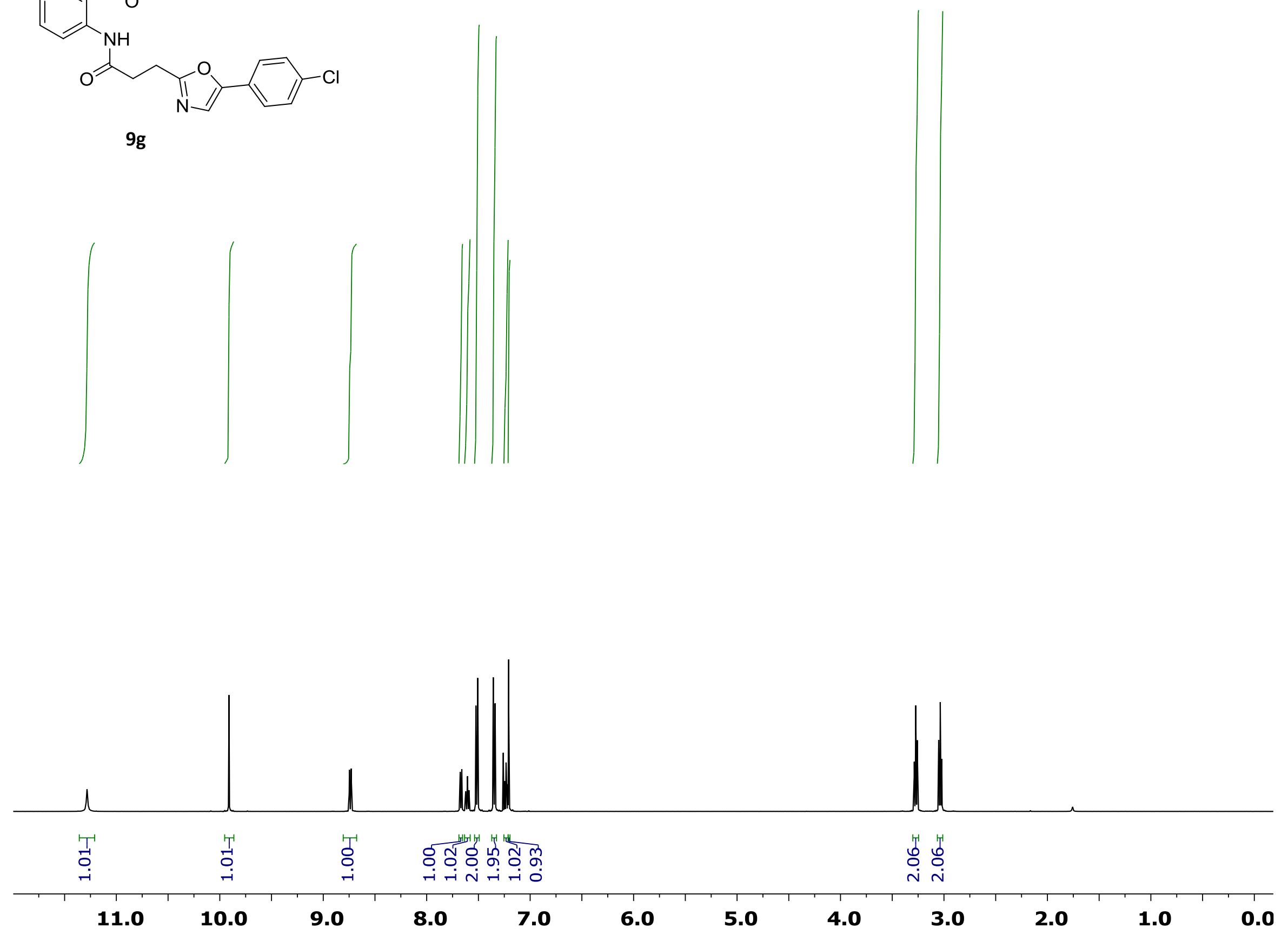

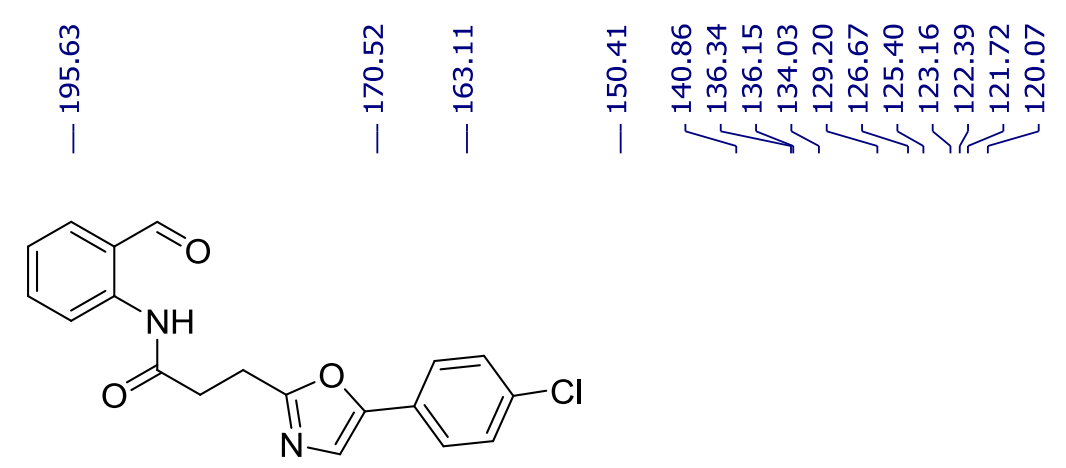

9g

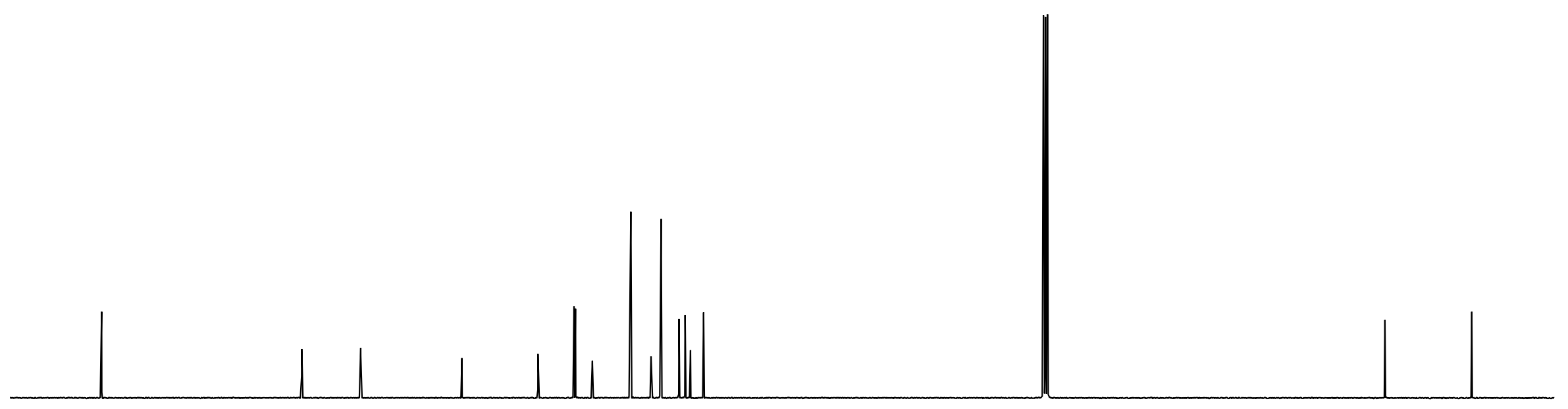




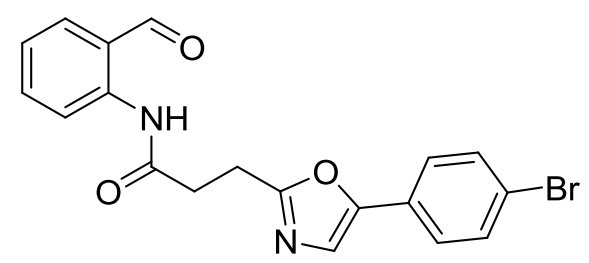

$9 h$
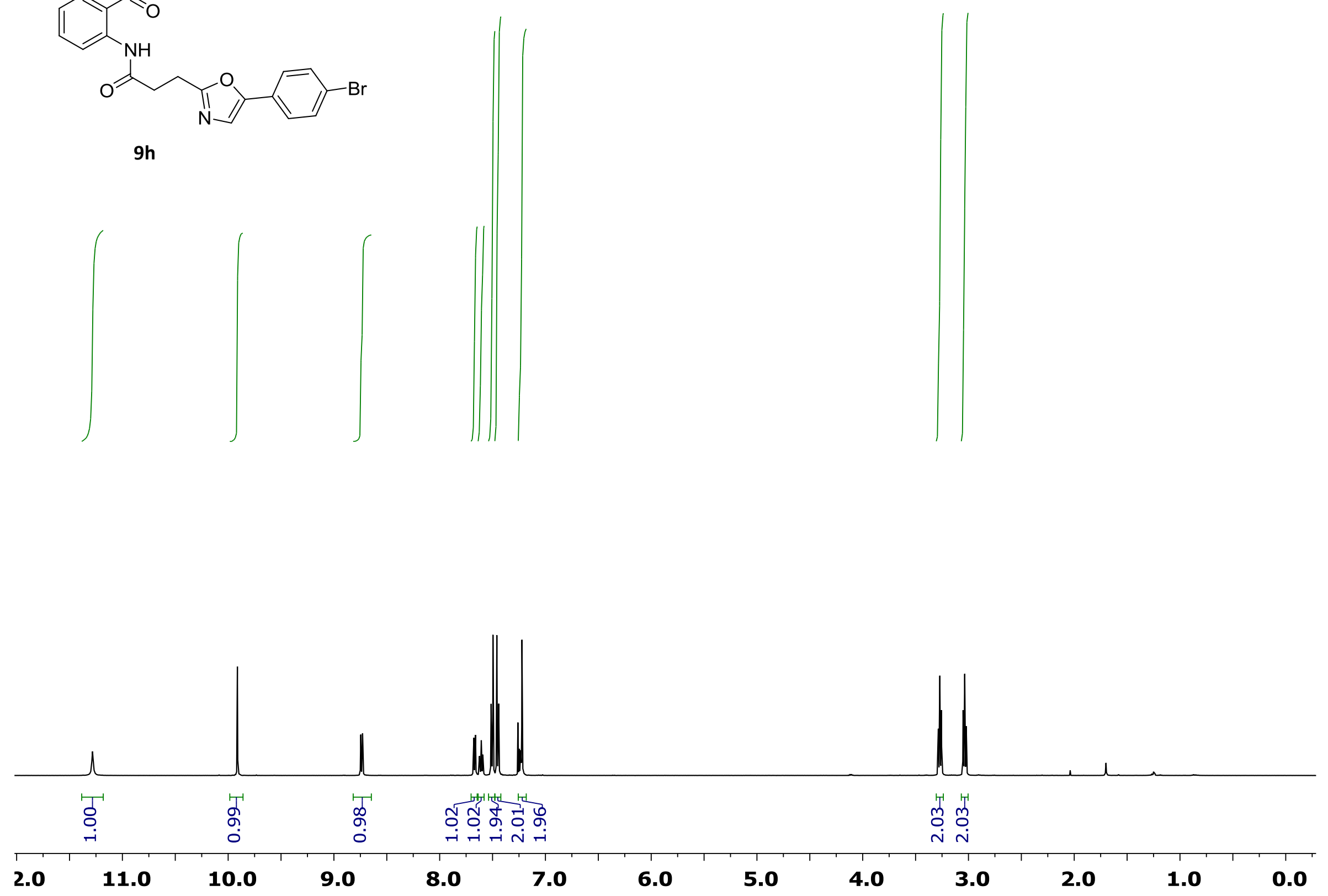


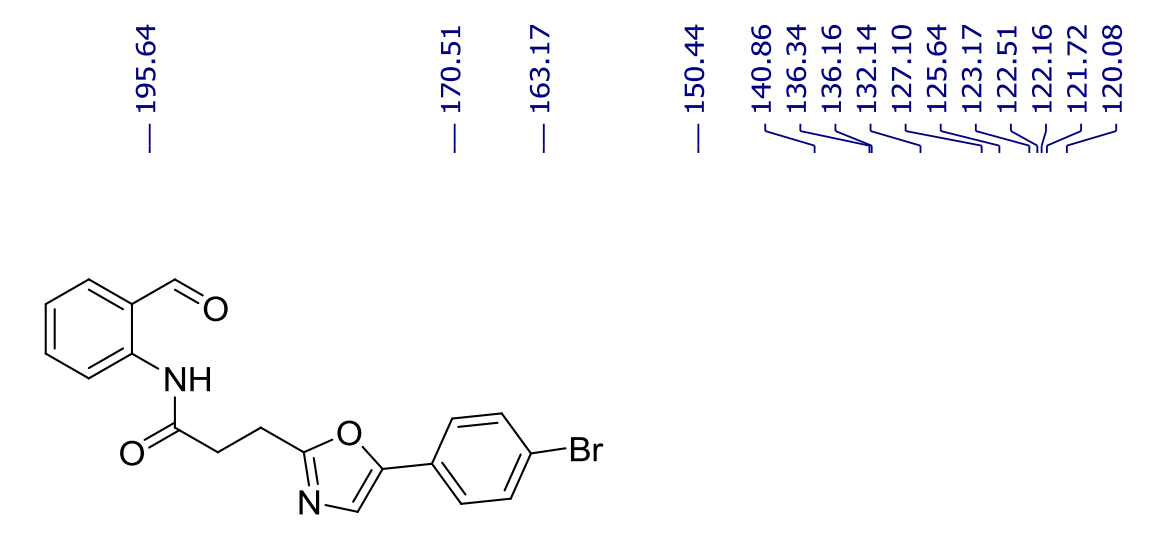

$9 h$

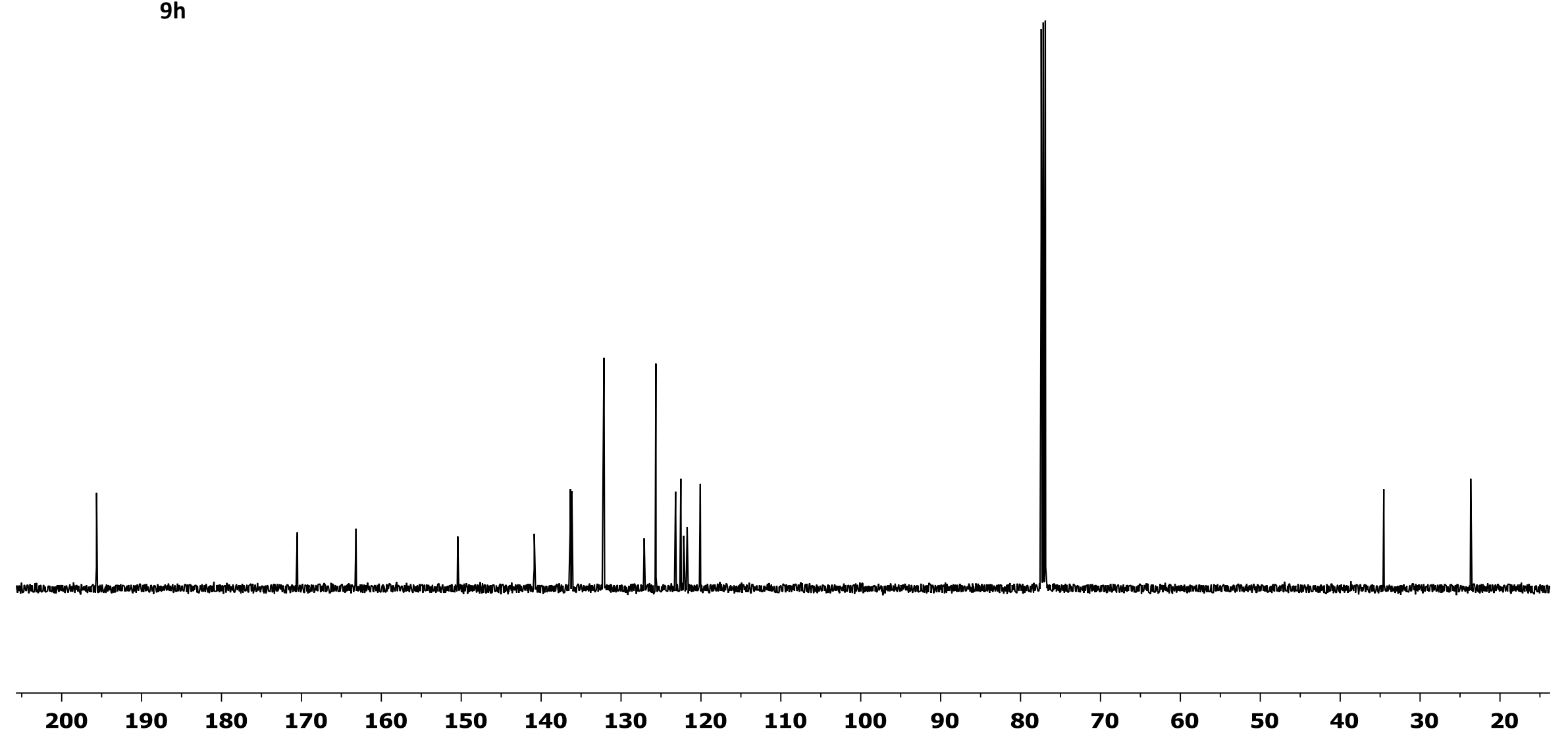




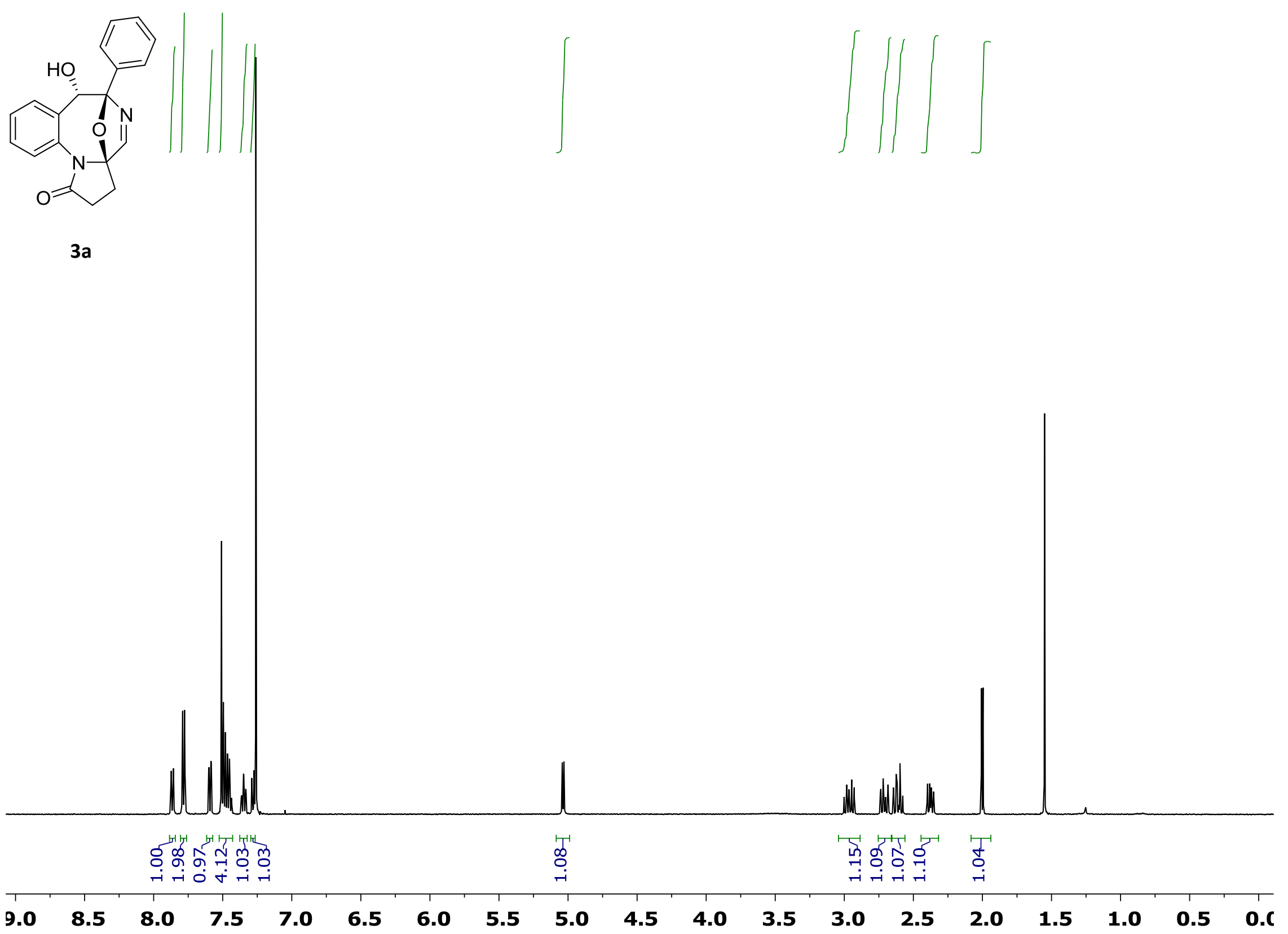




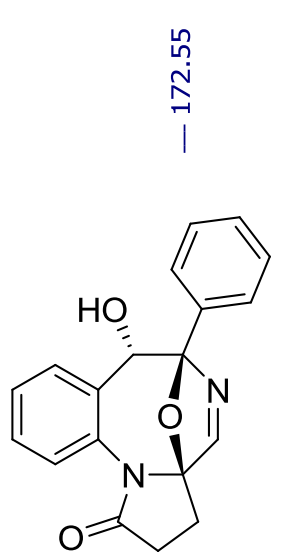

$$
\text { | }
$$

Ln :

mे

3a

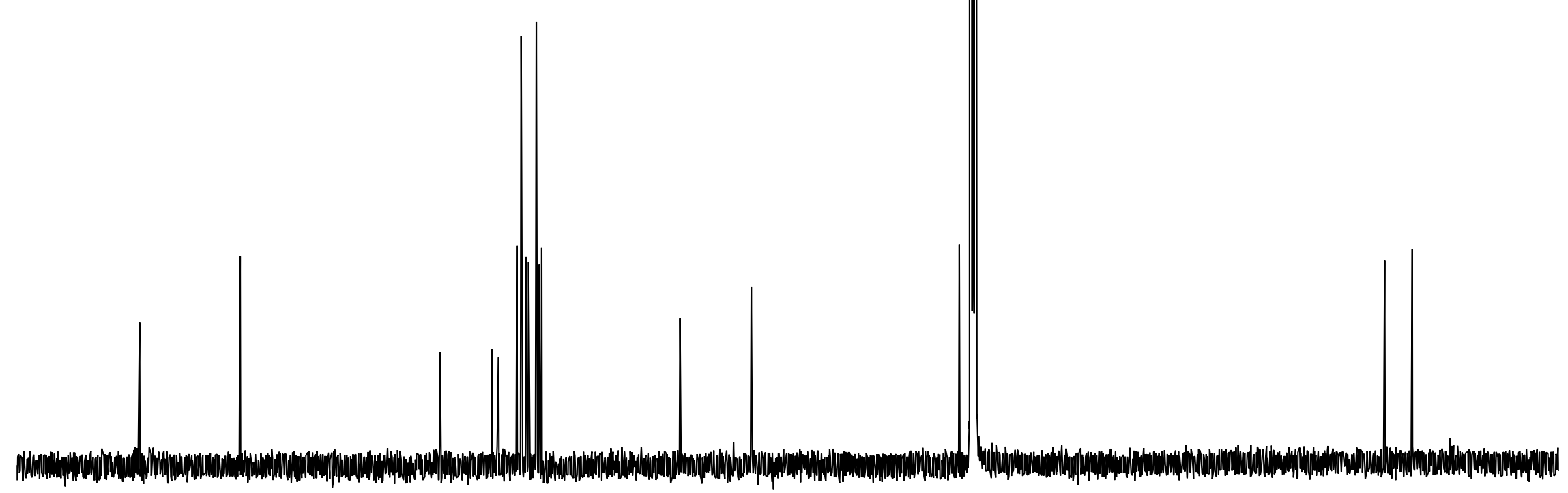

$180 \quad 170$

160

150

140

130

120

110100

90

80

70

60

50

40

30 


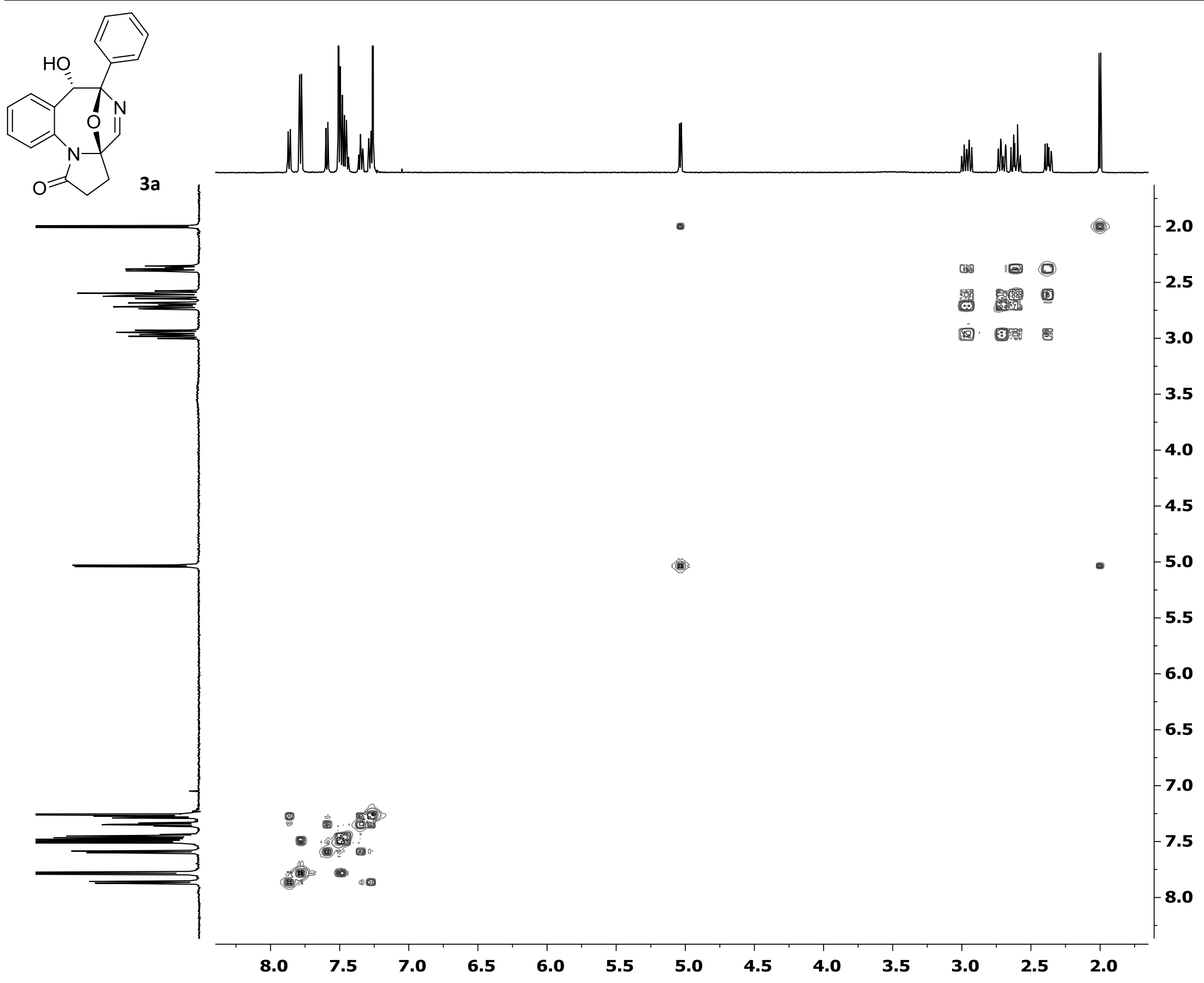




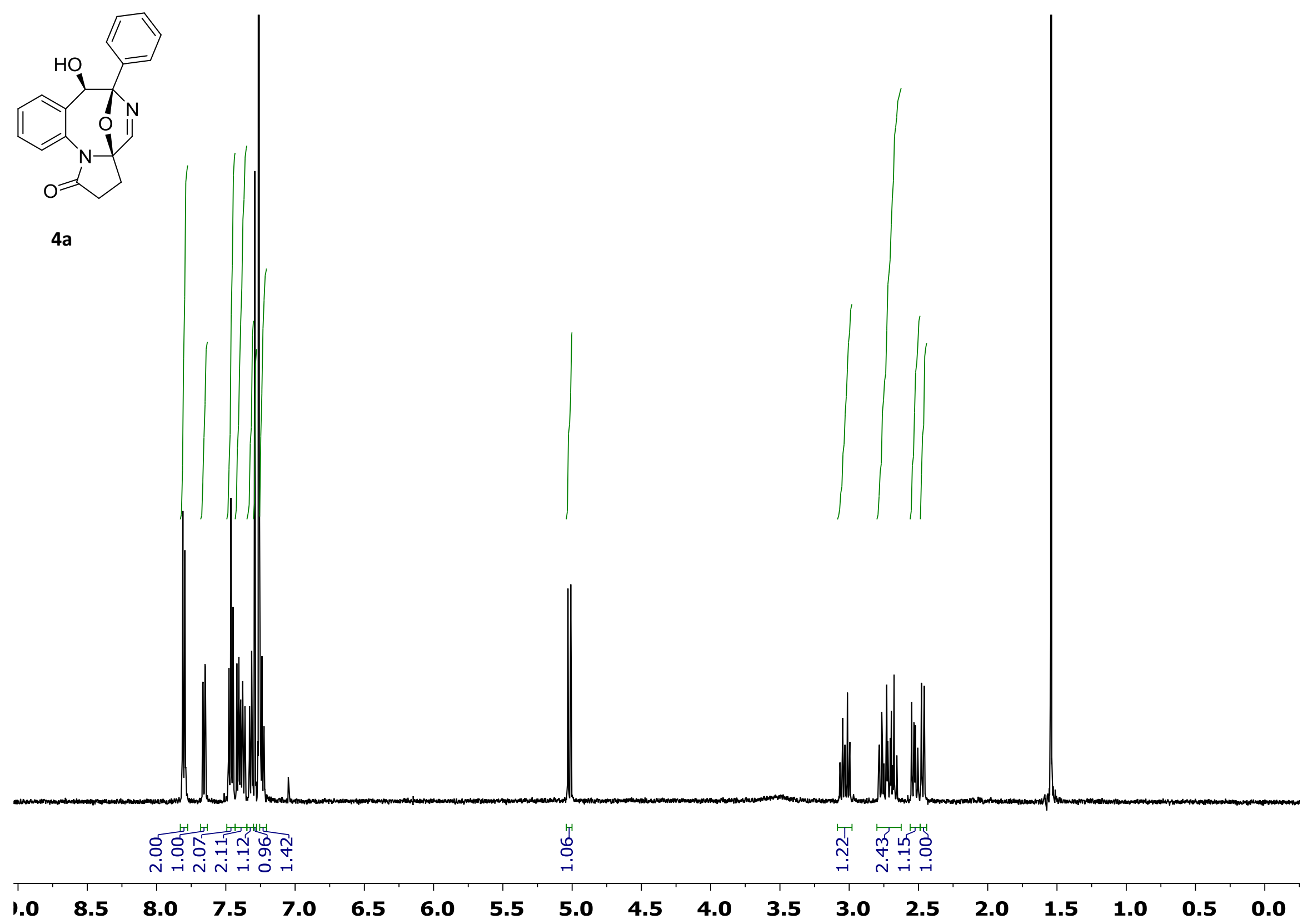




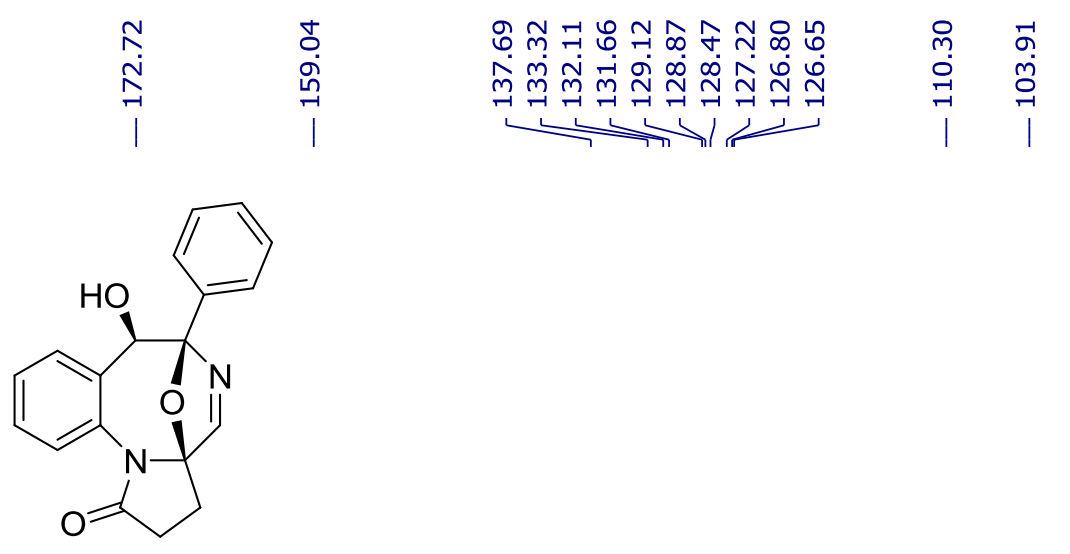

$4 a$
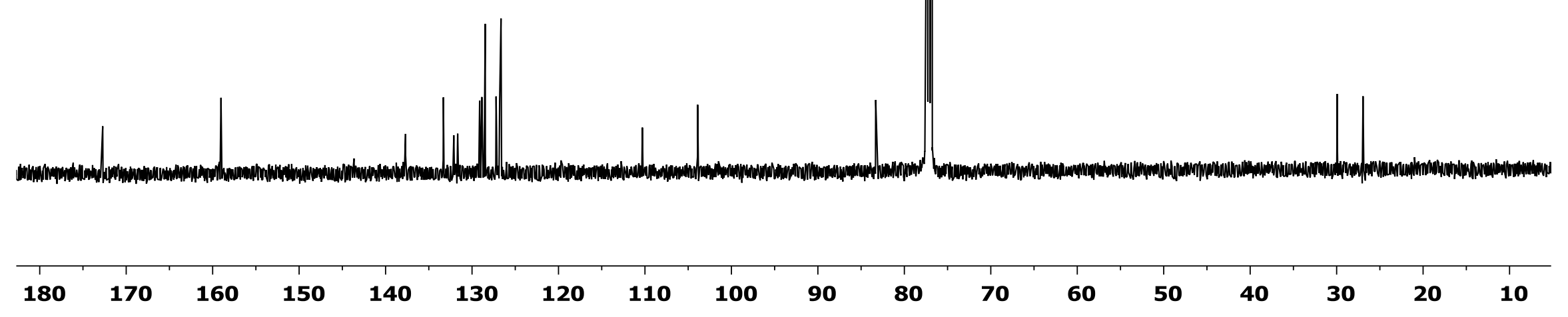


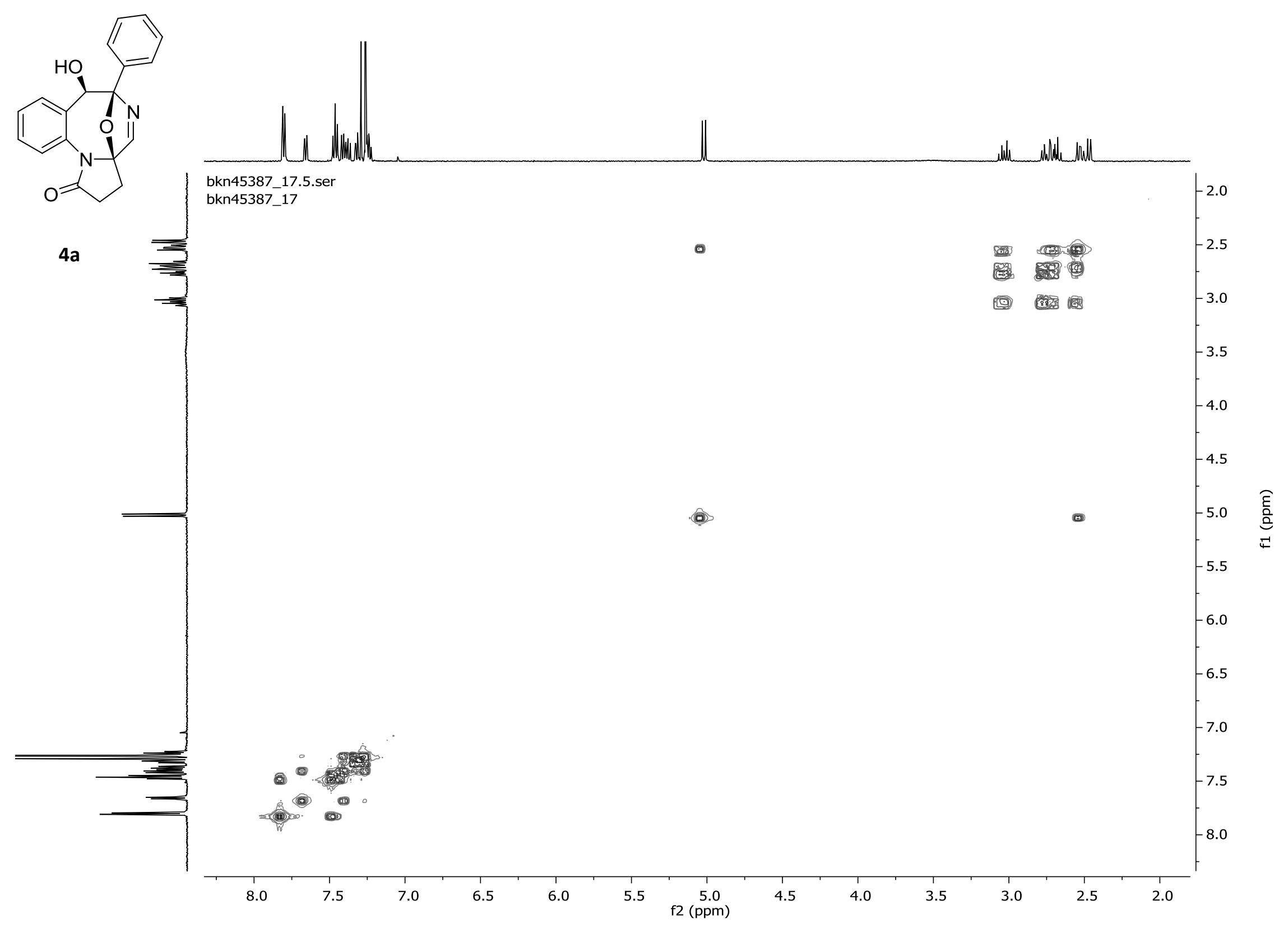




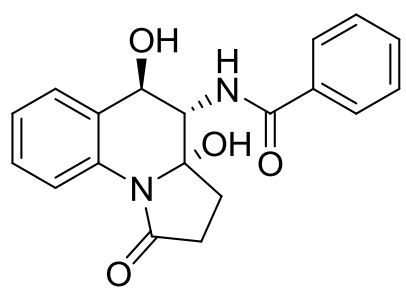

$5 a$
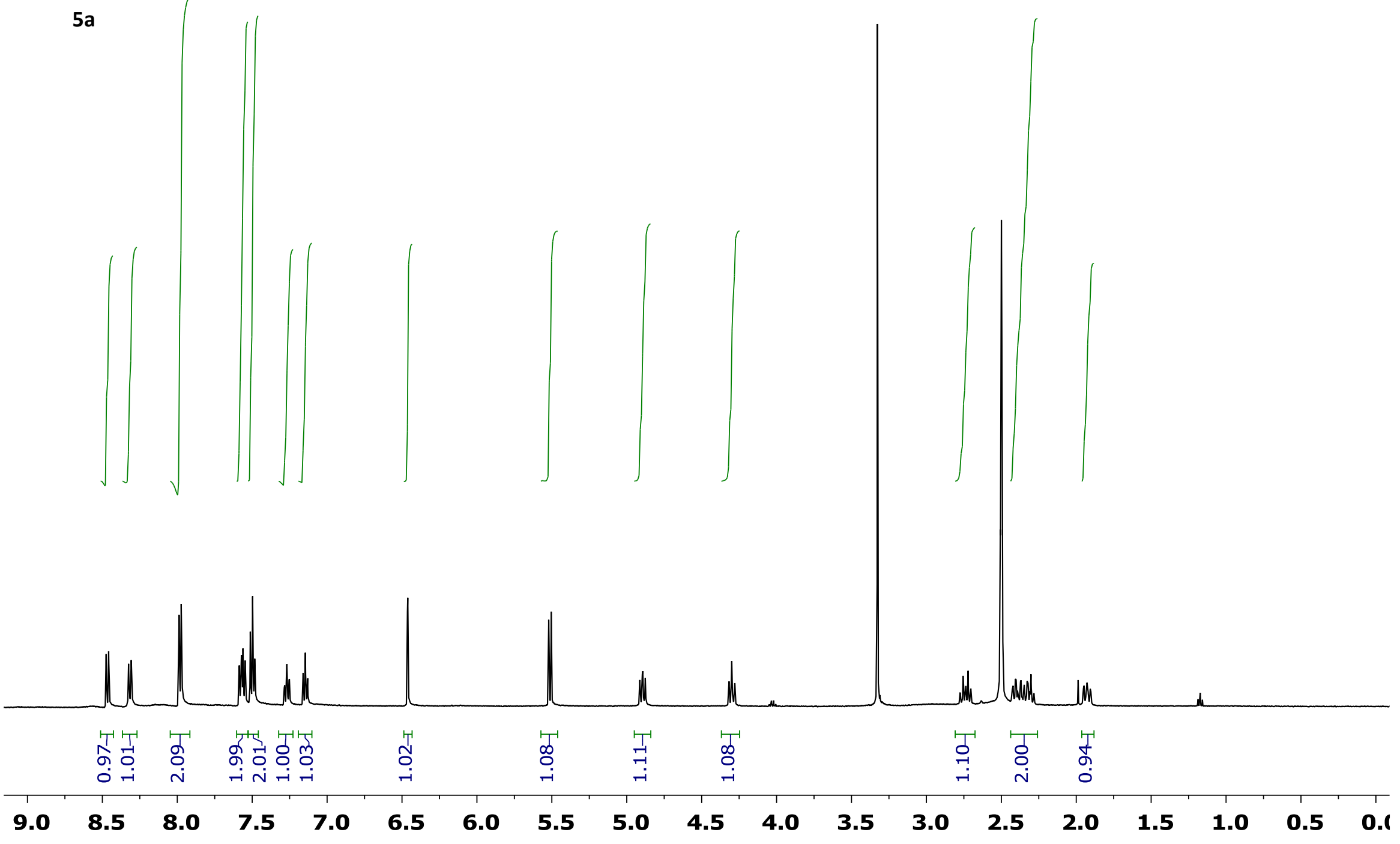


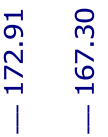

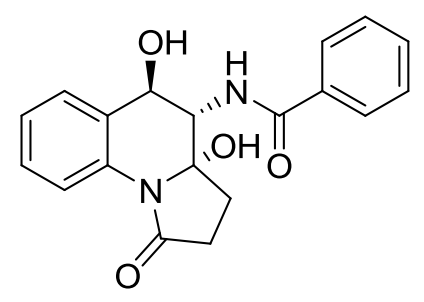

$5 a$

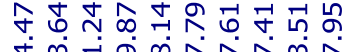

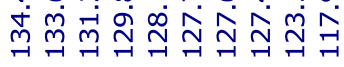

|

\begin{tabular}{l}
+ \\
$\infty$ \\
8 \\
\hline
\end{tabular}

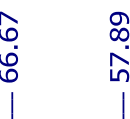

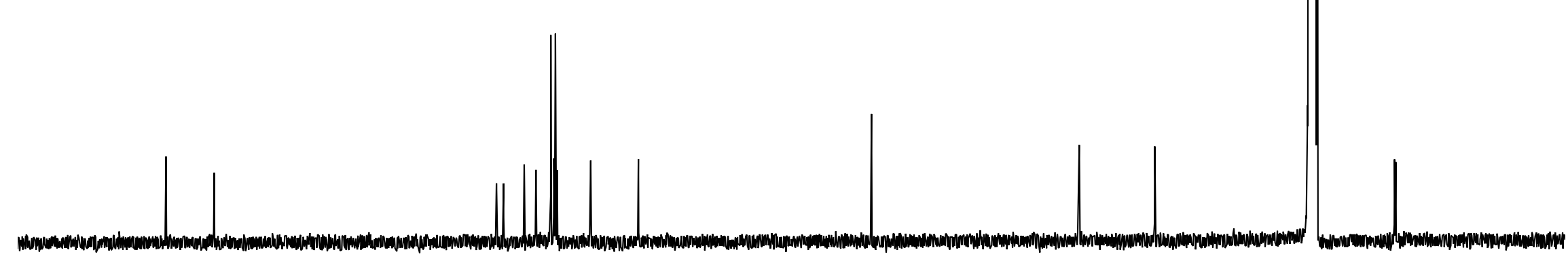




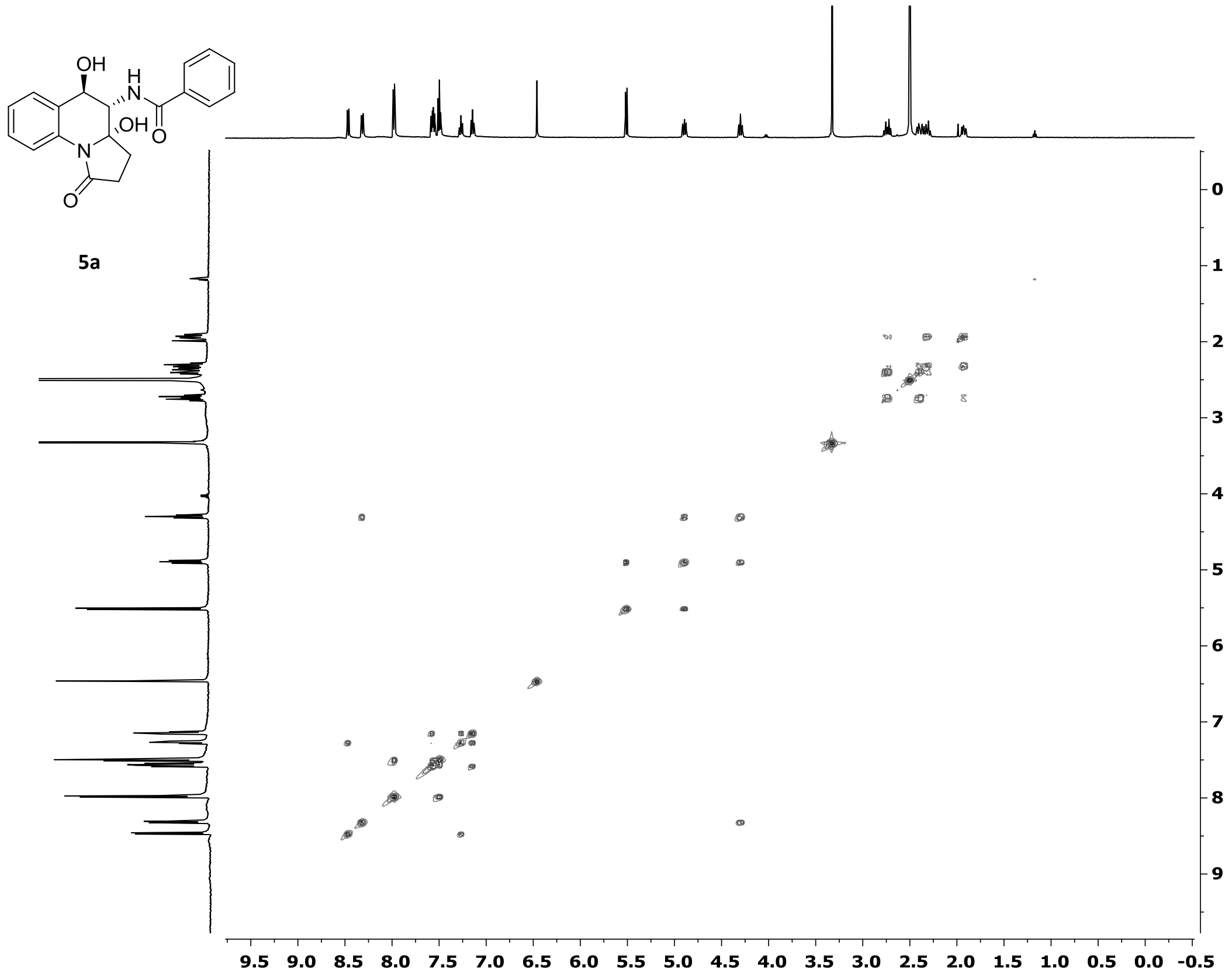



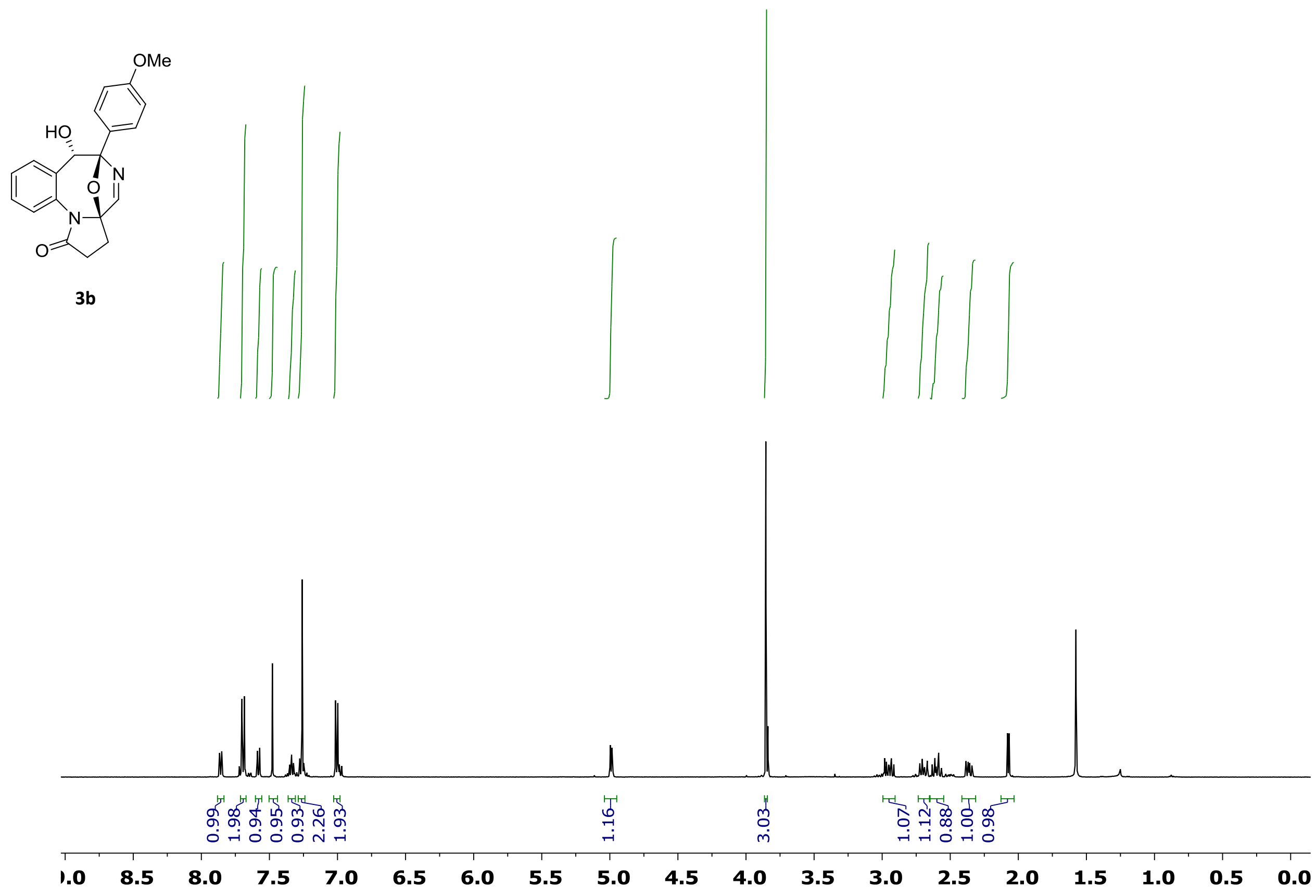

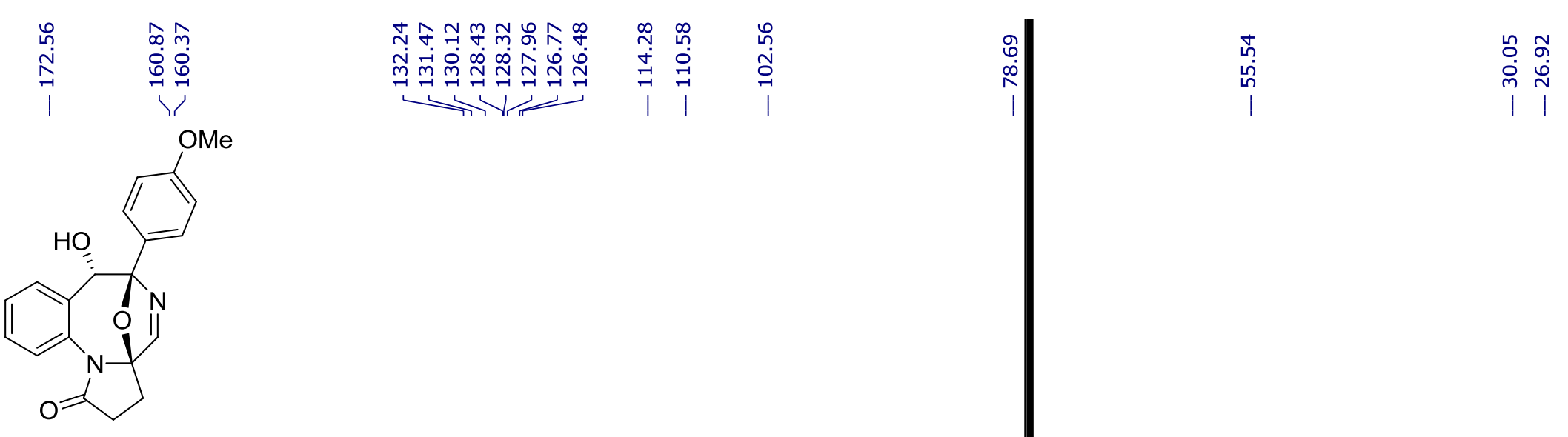

$3 b$

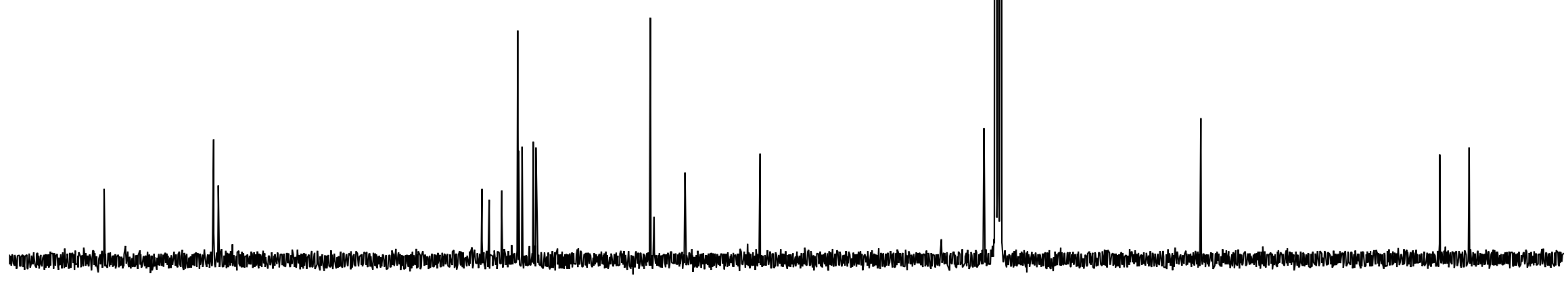




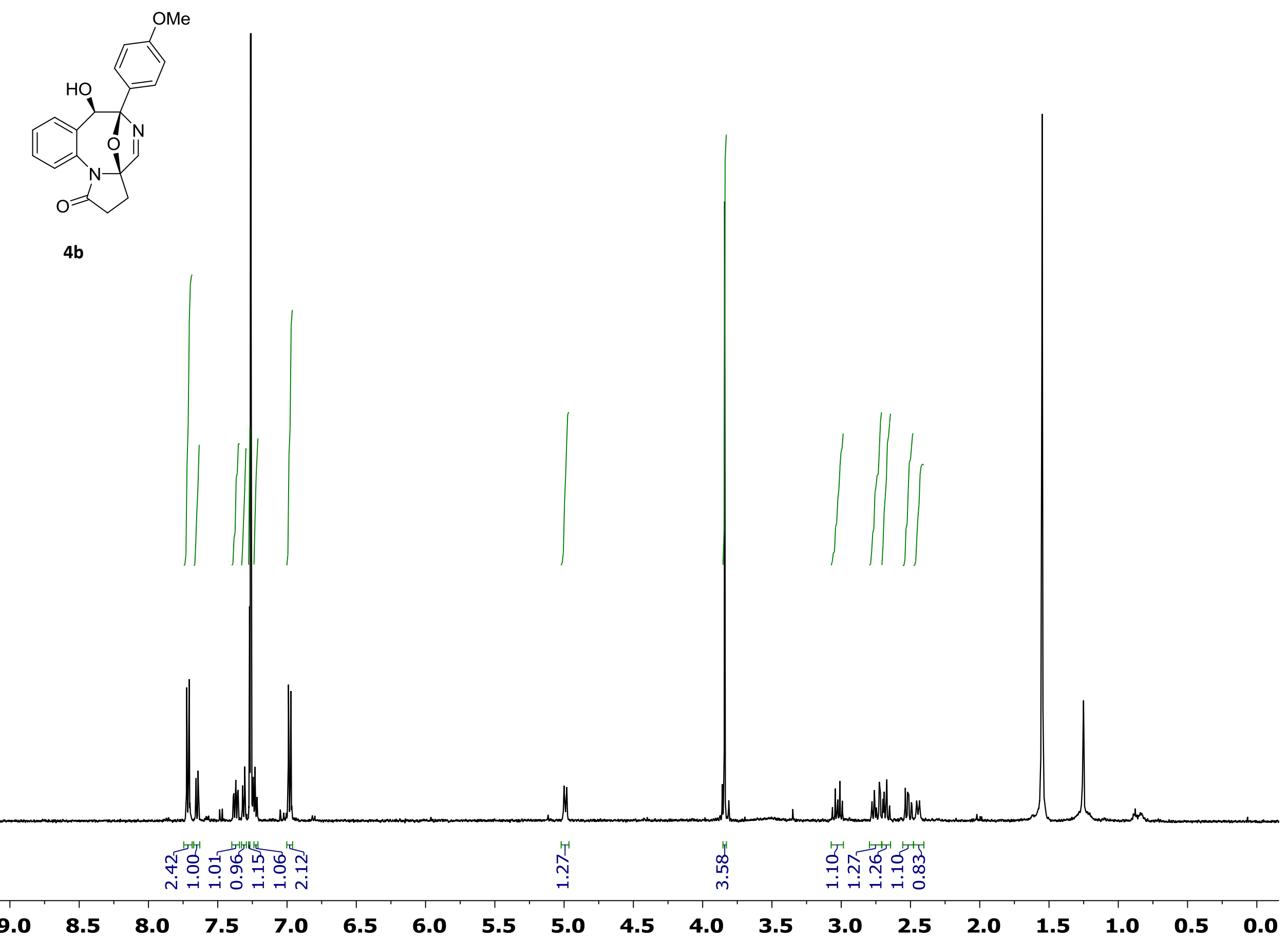




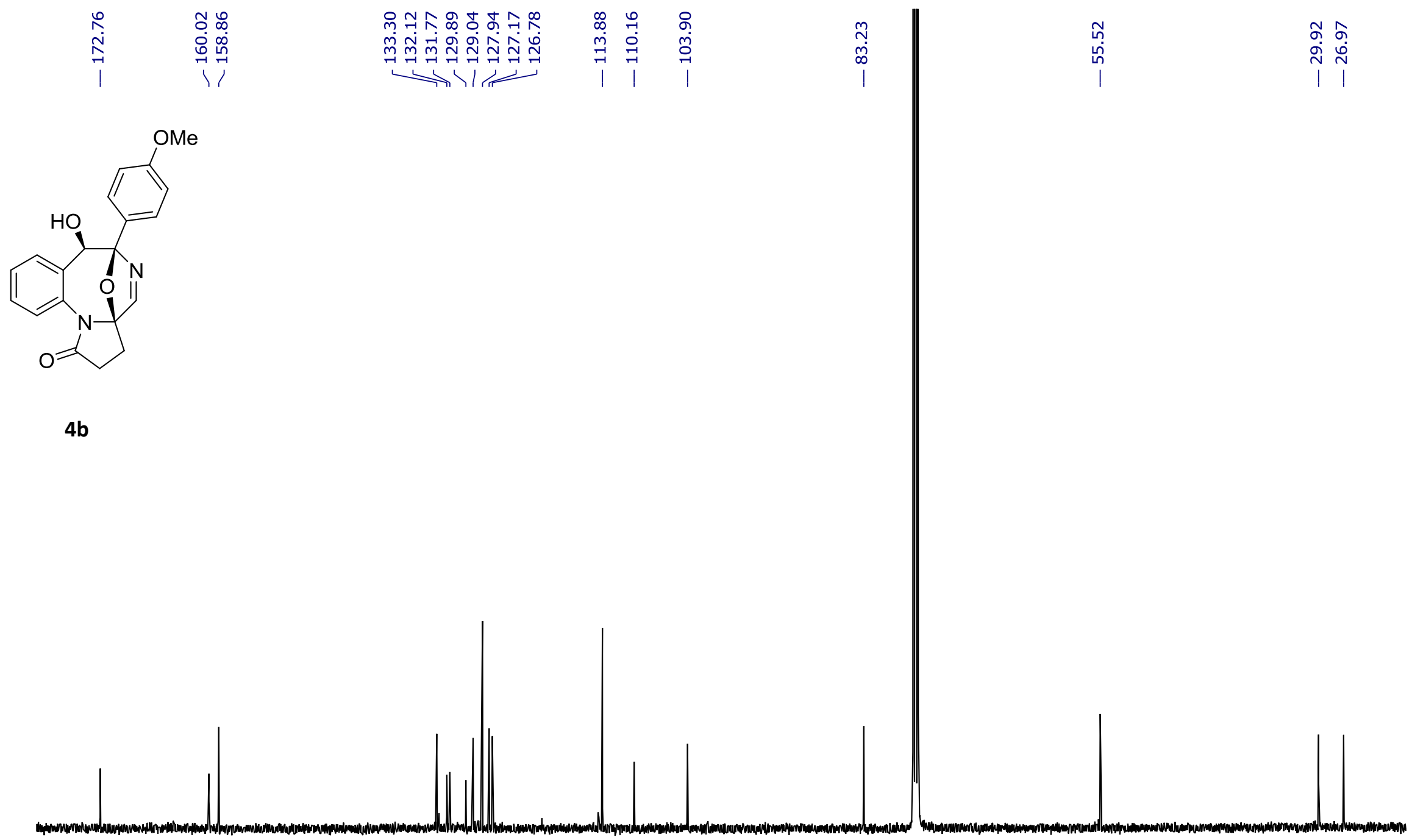

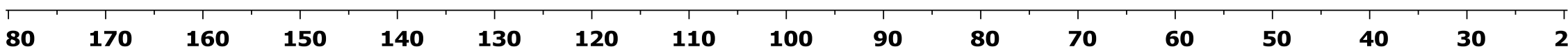


<smiles>COc1ccc(C(=O)N[C@H]2[C@H](O)c3ccccc3N3C(=O)CC[C@]23O)cc1</smiles>

$5 b$
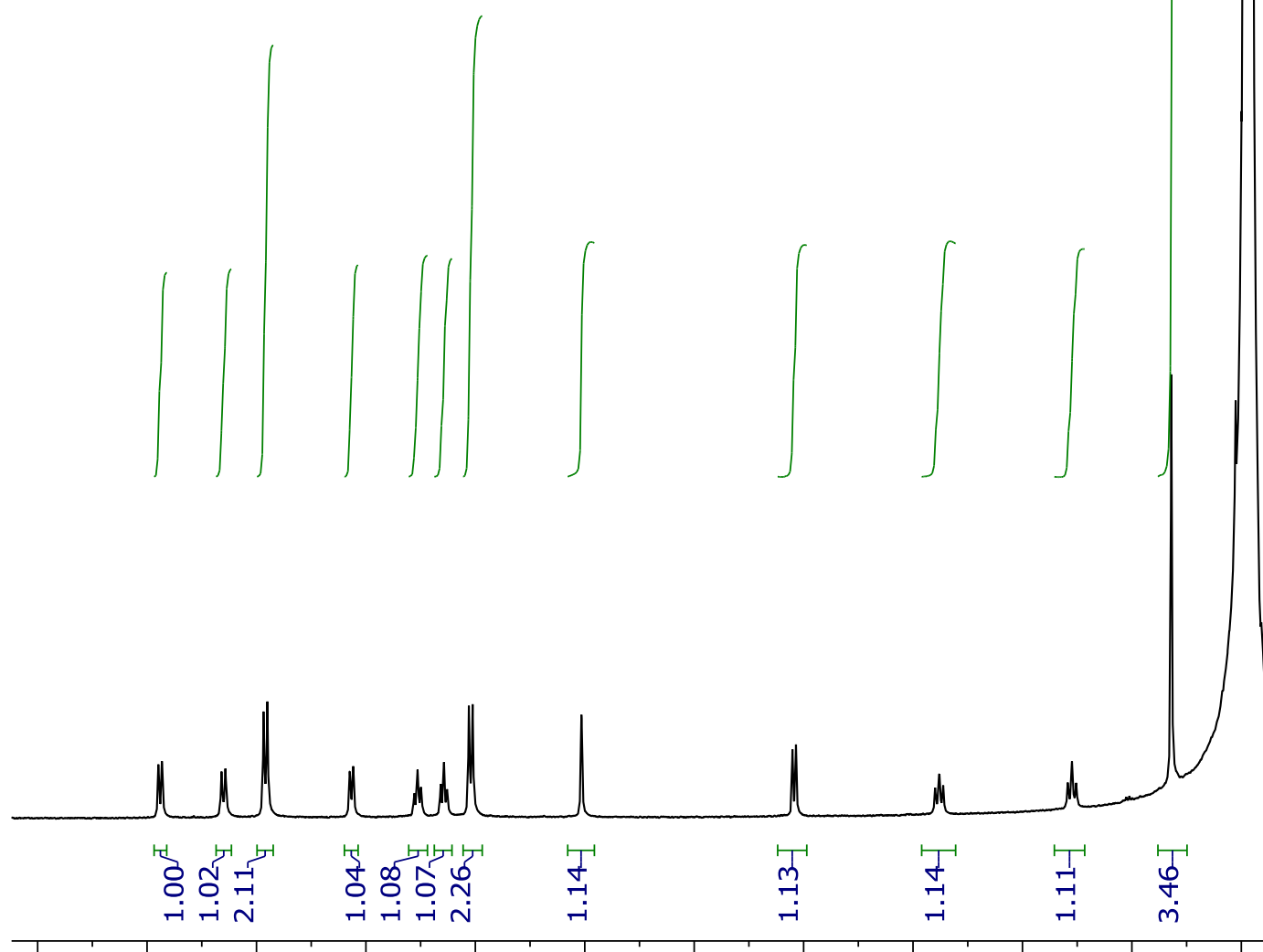

$\begin{array}{llllllllll}9.0 & 8.5 & 8.0 & 7.5 & 7.0 & 6.5 & 6.0 & 5.5 & 5.0 & 4.5\end{array}$

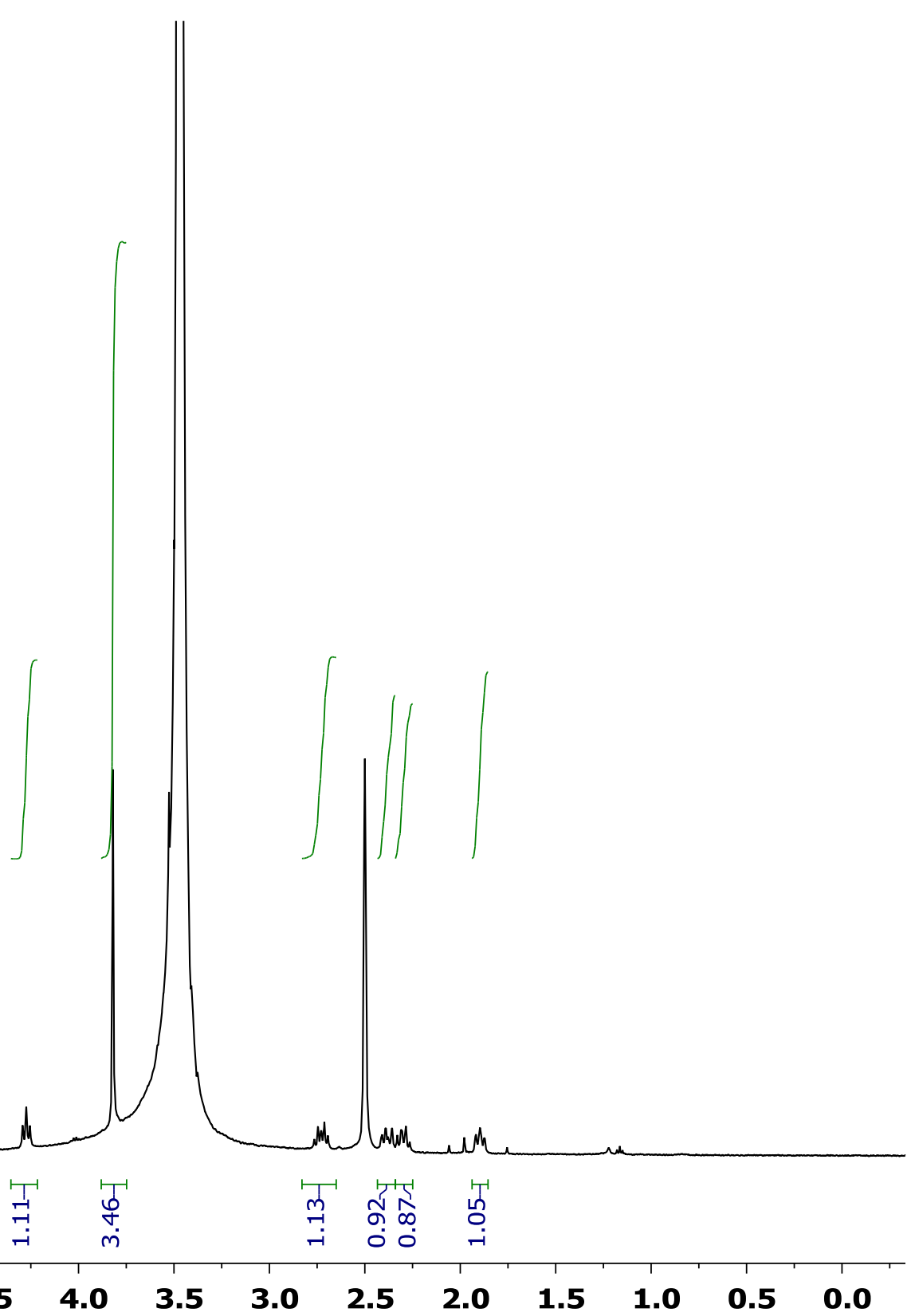




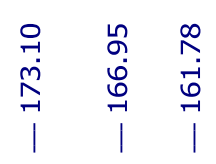

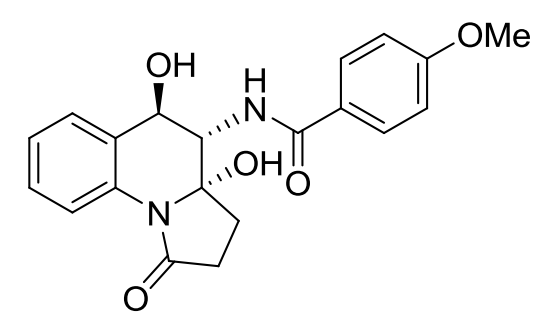

$5 b$

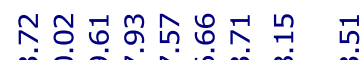

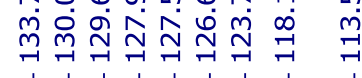 \\ ।}

¿্ 


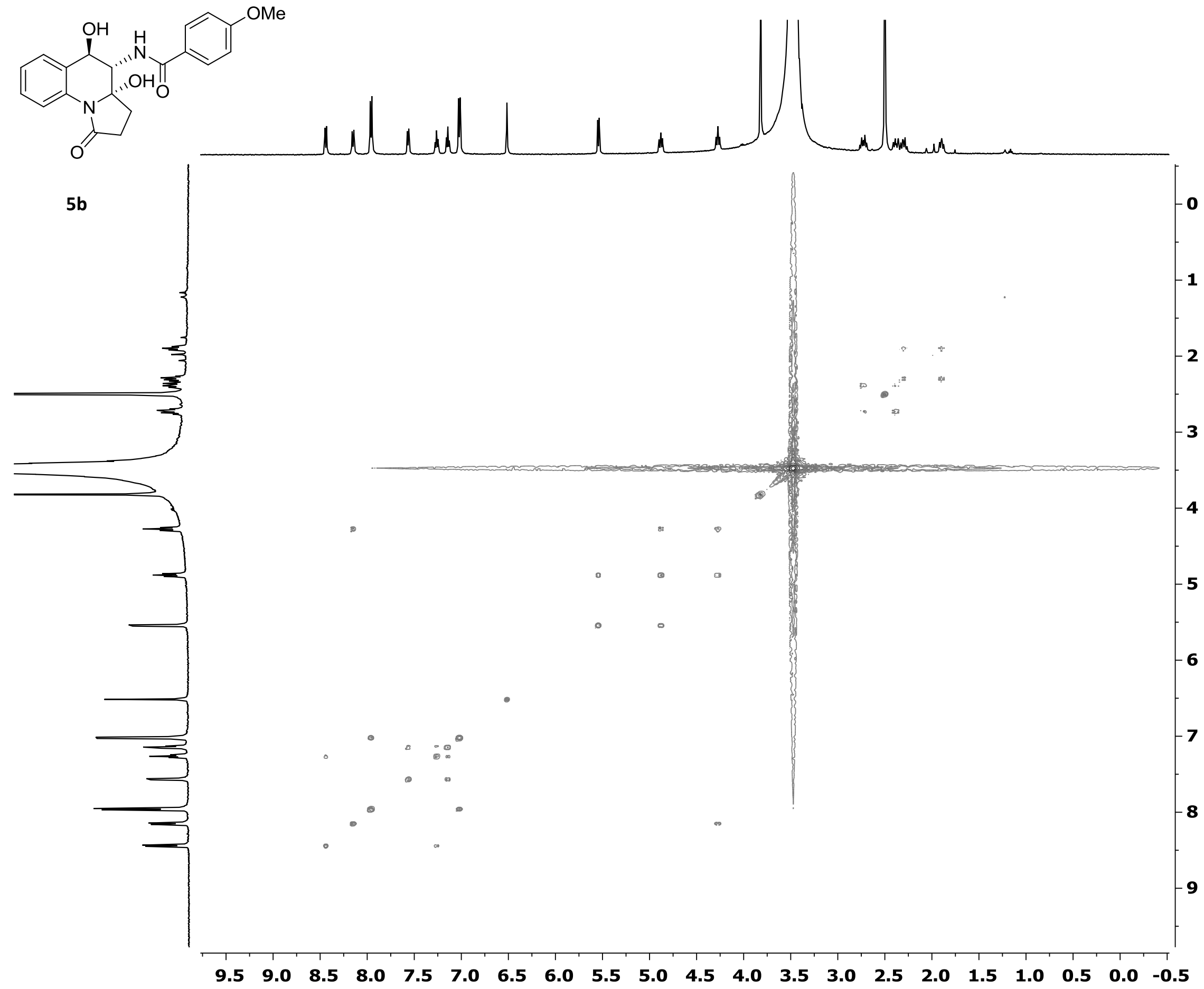




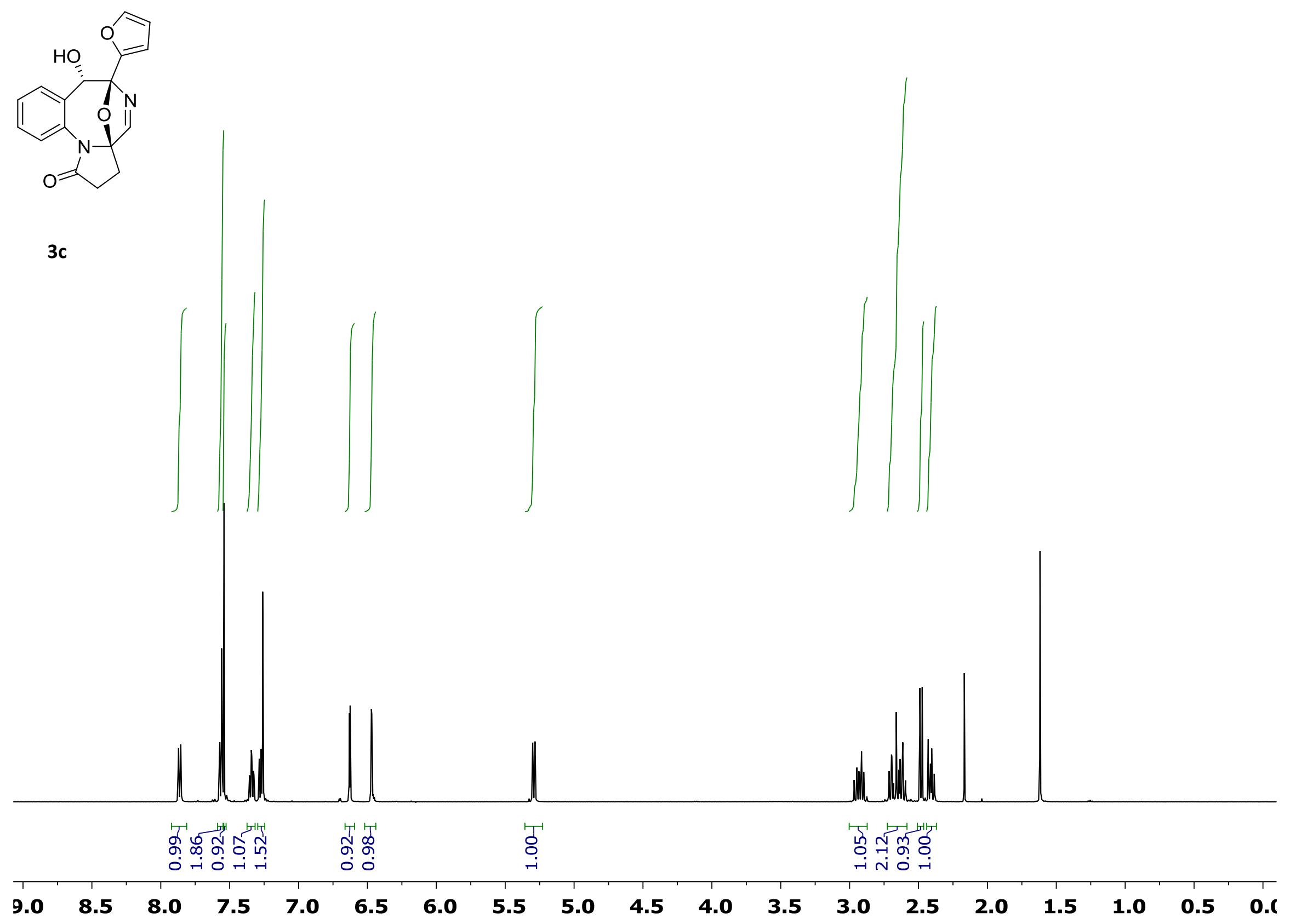



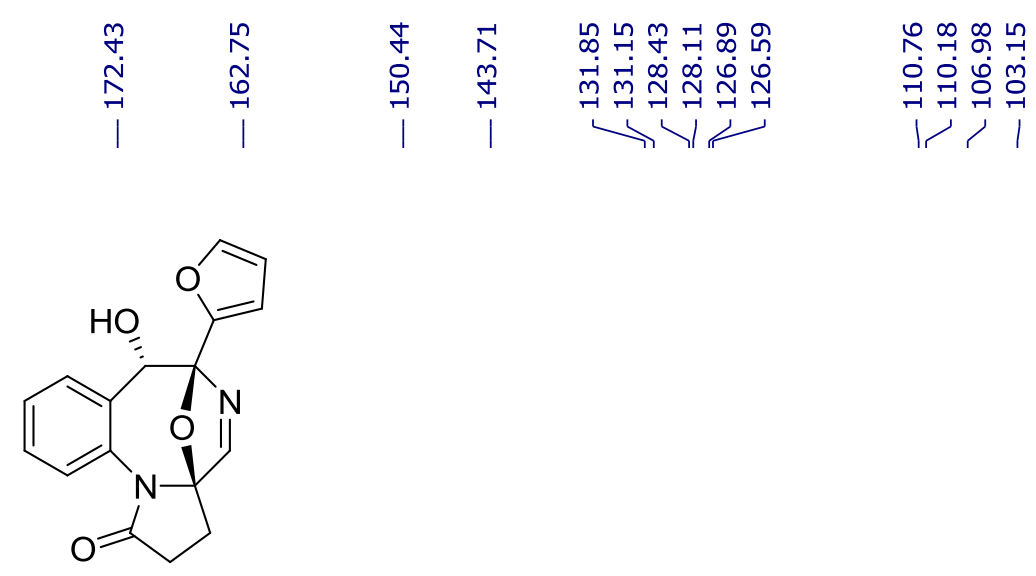

$3 c$

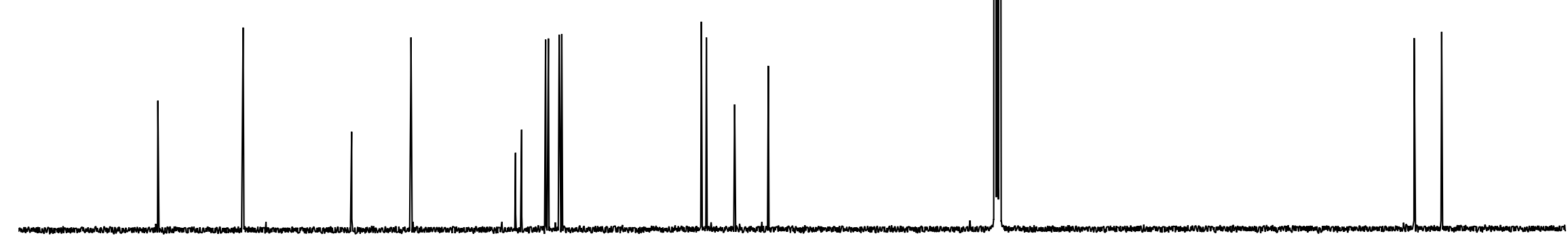




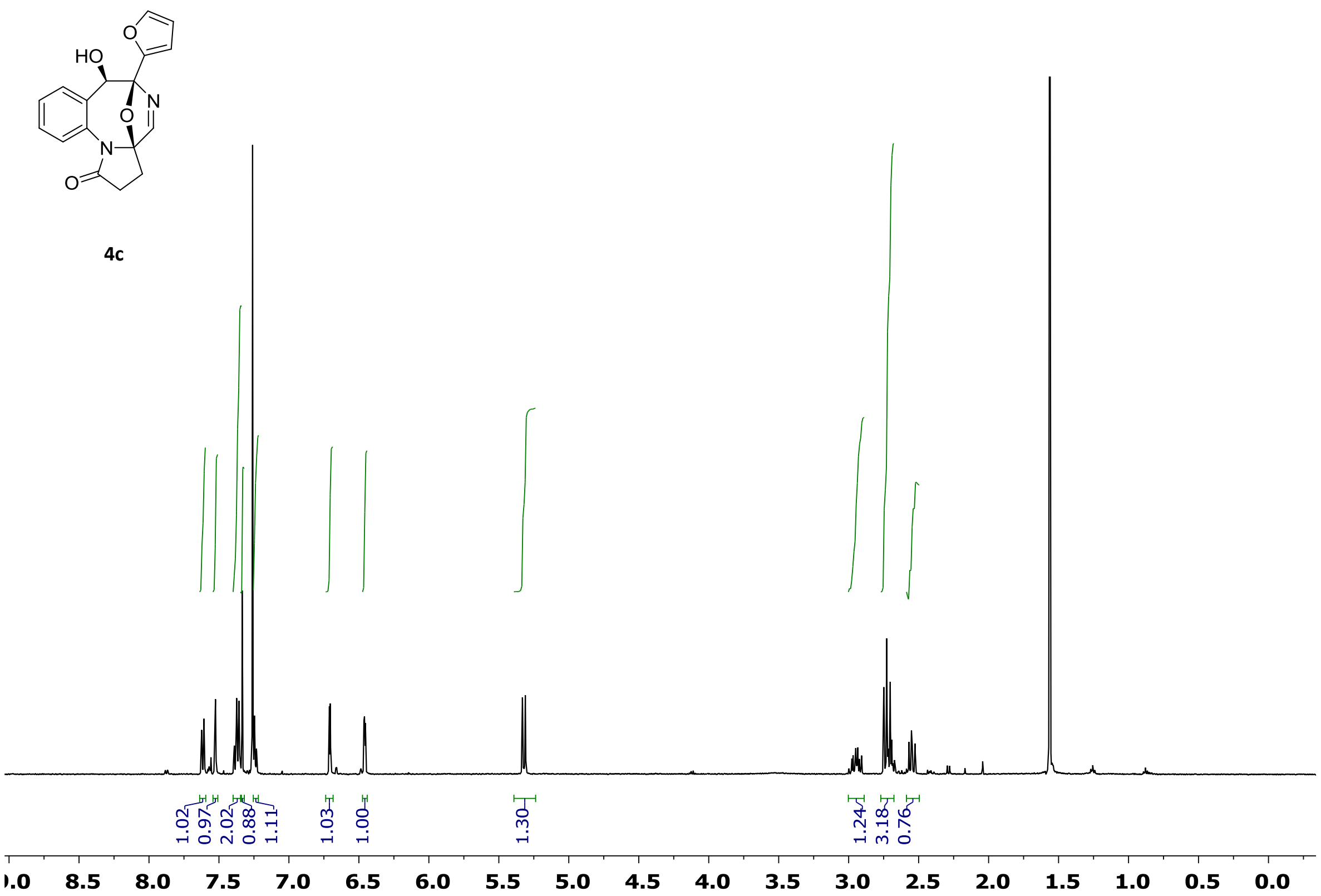




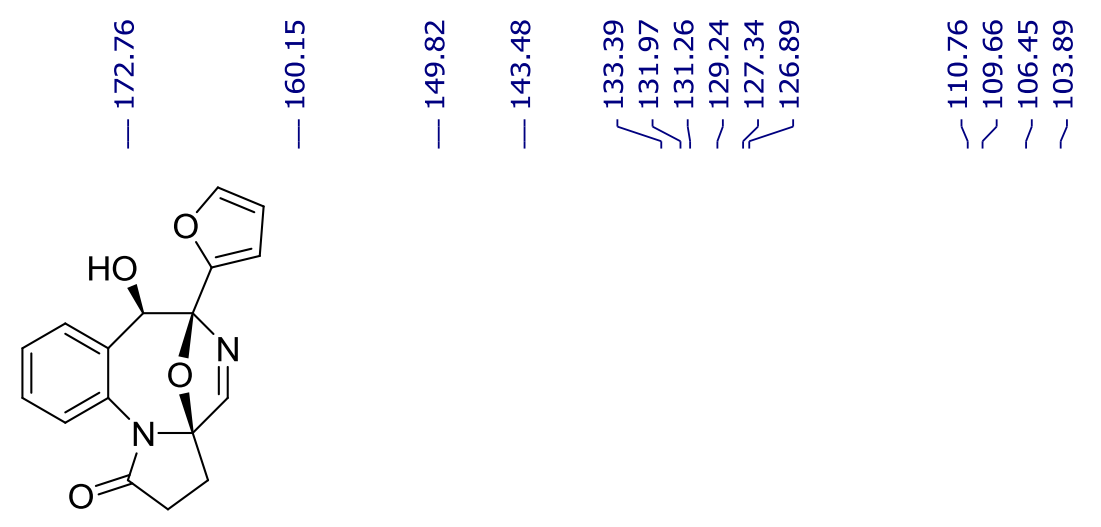

$4 c$
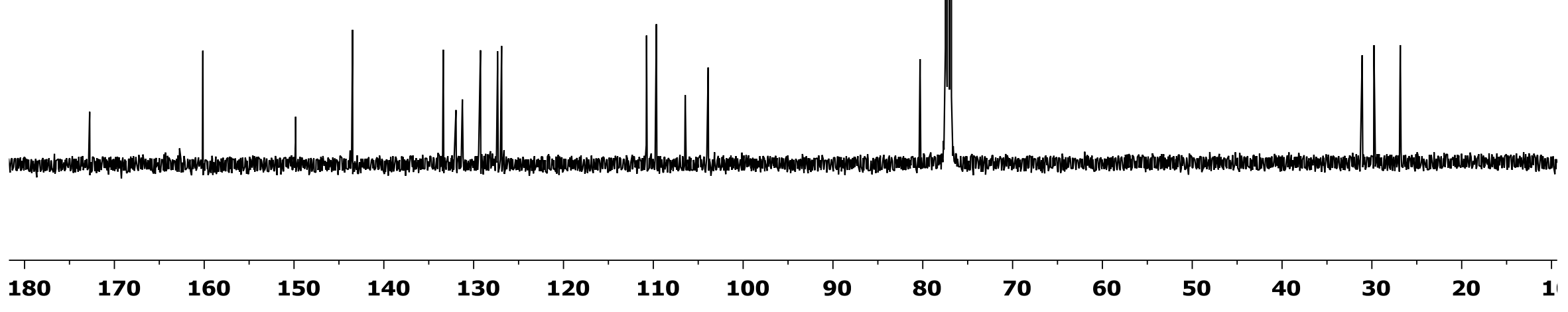


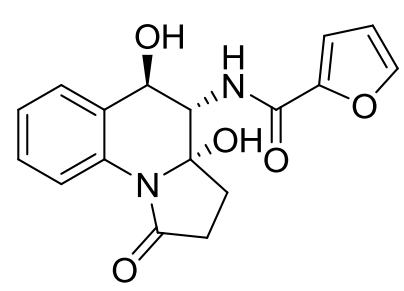

$5 c$
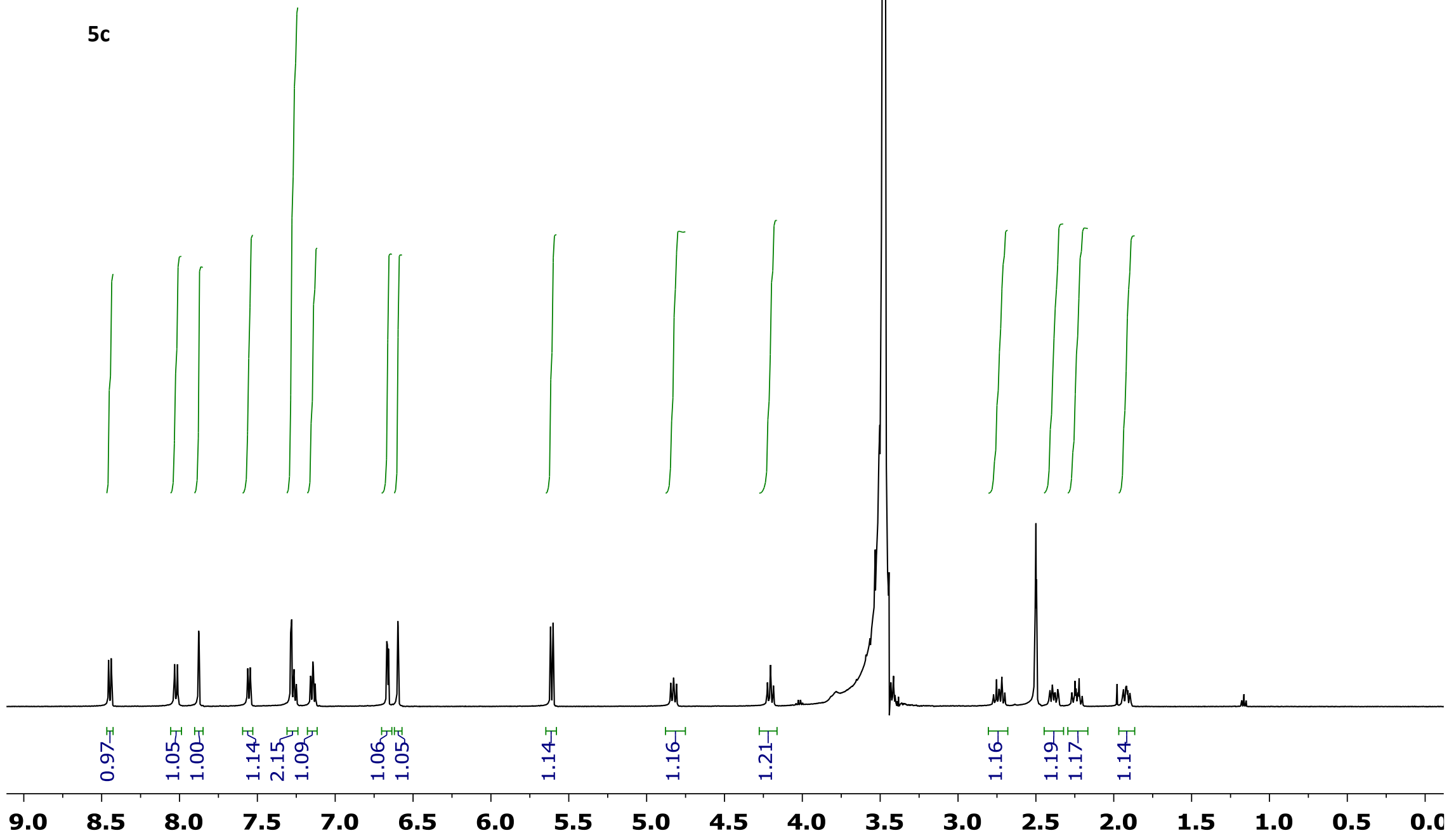


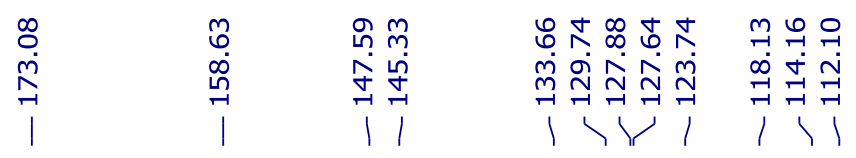

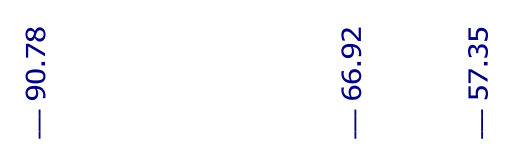

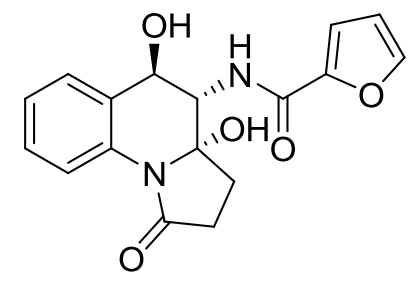

$5 c$

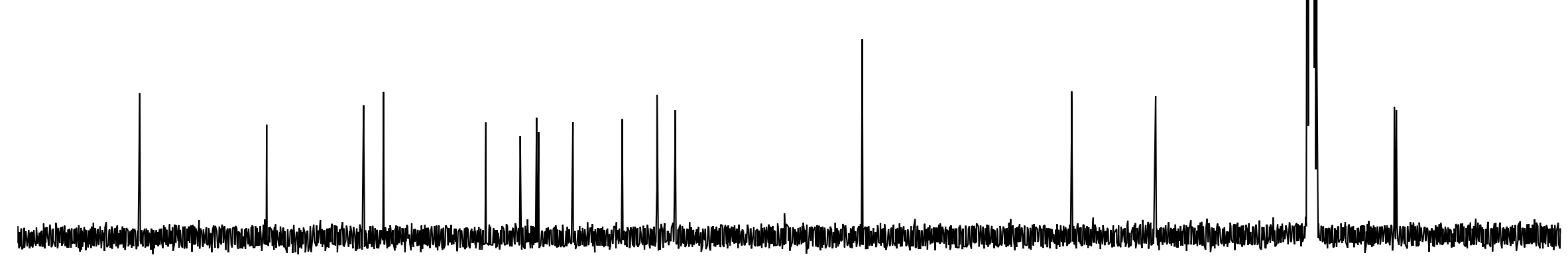

$180 \quad 170 \quad 160 \quad 150$

$140 \quad 130 \quad 120$

110

100

90

80

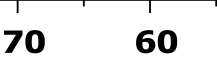

50

$40 \quad 30$ 


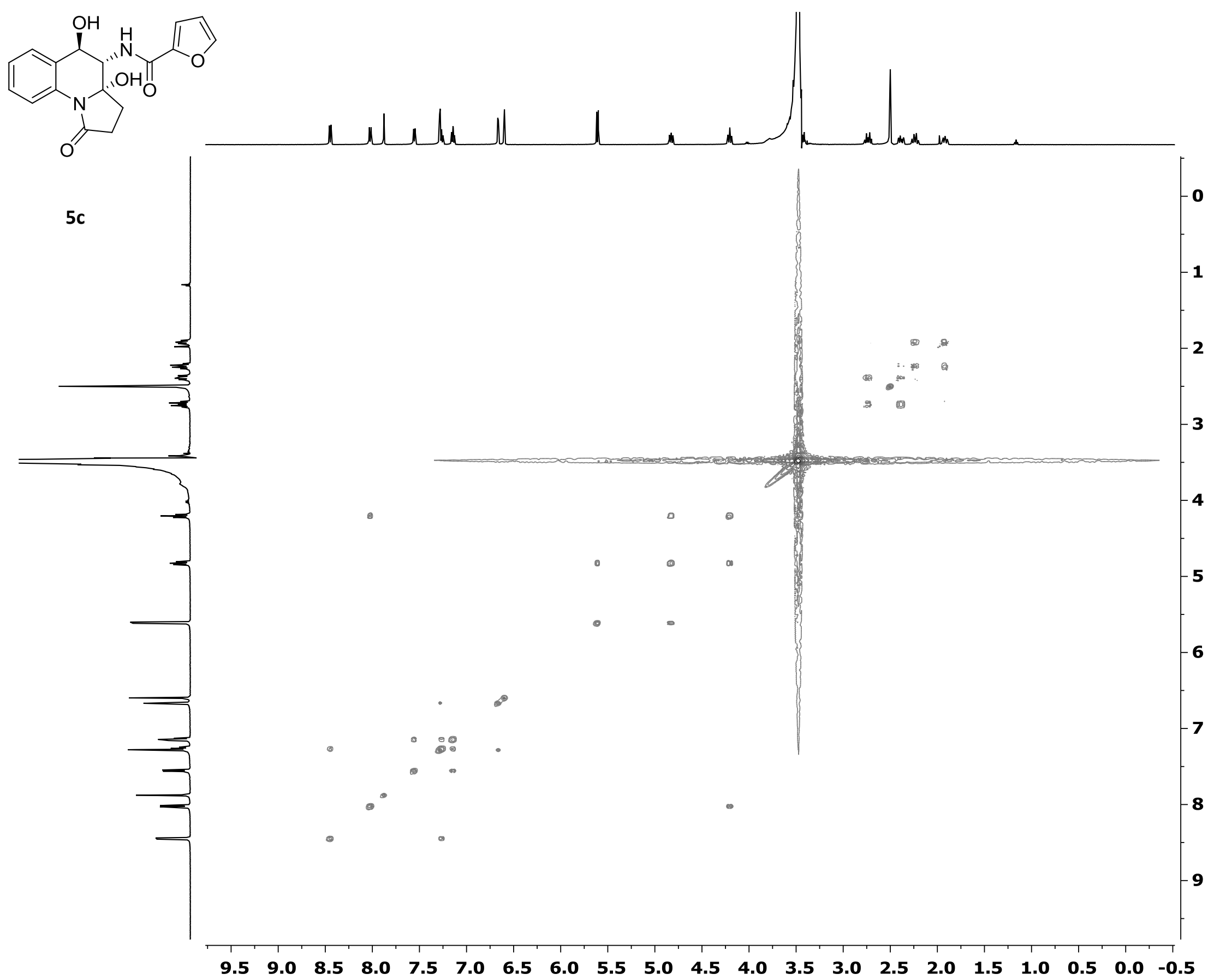



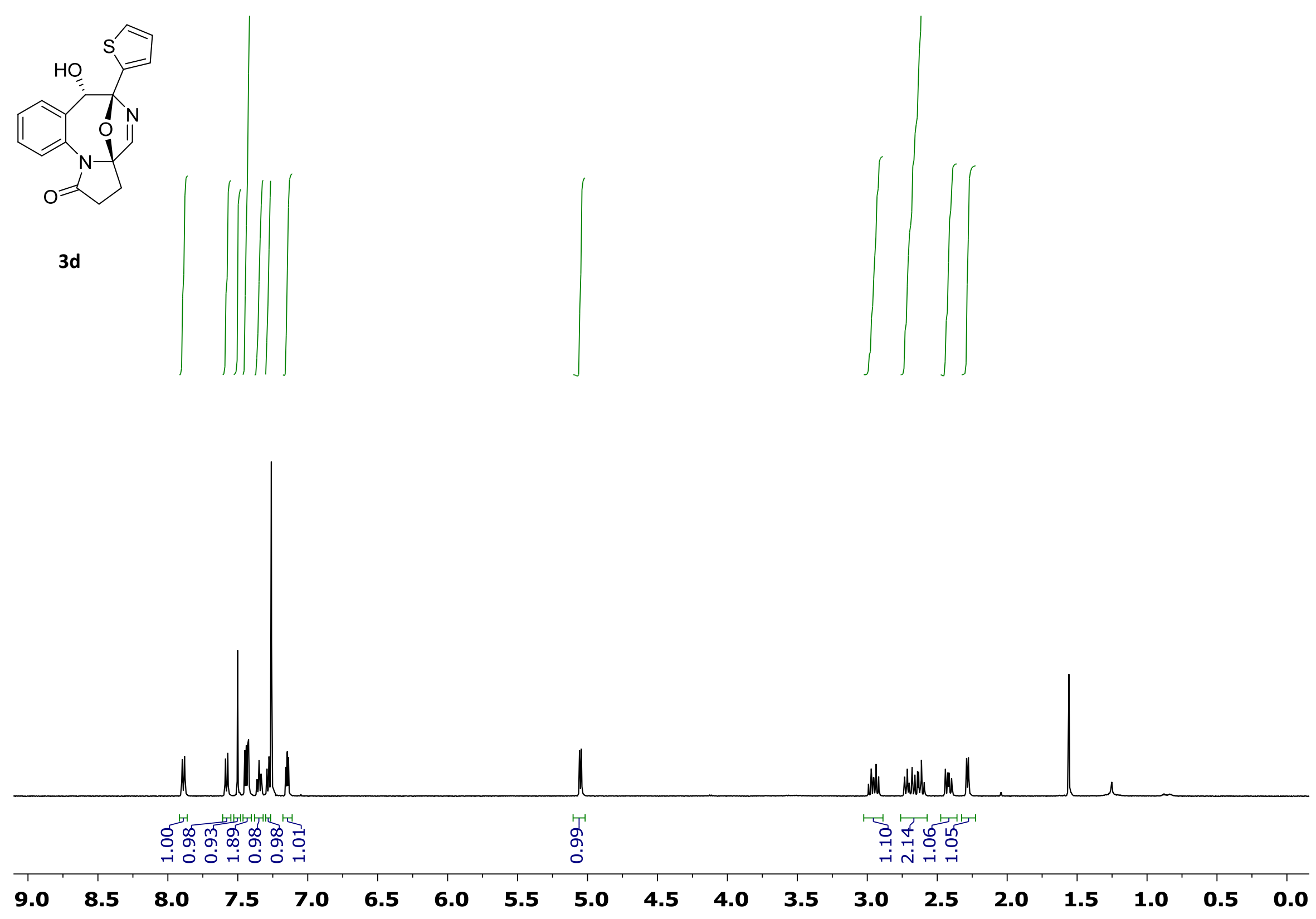


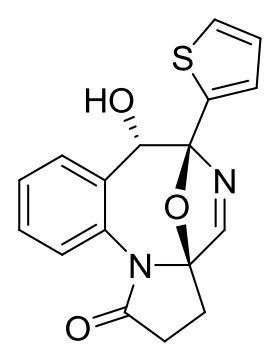

3d
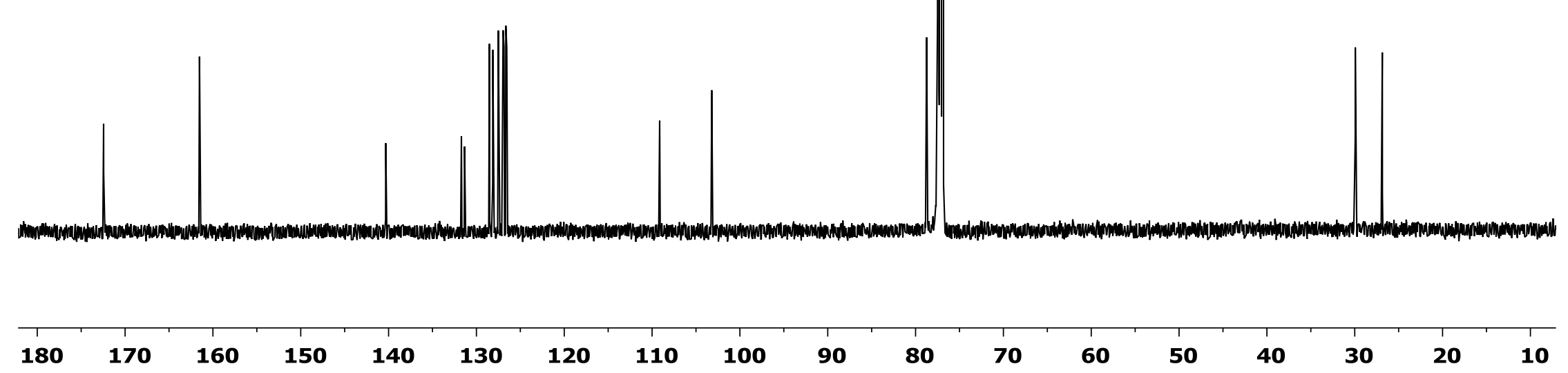


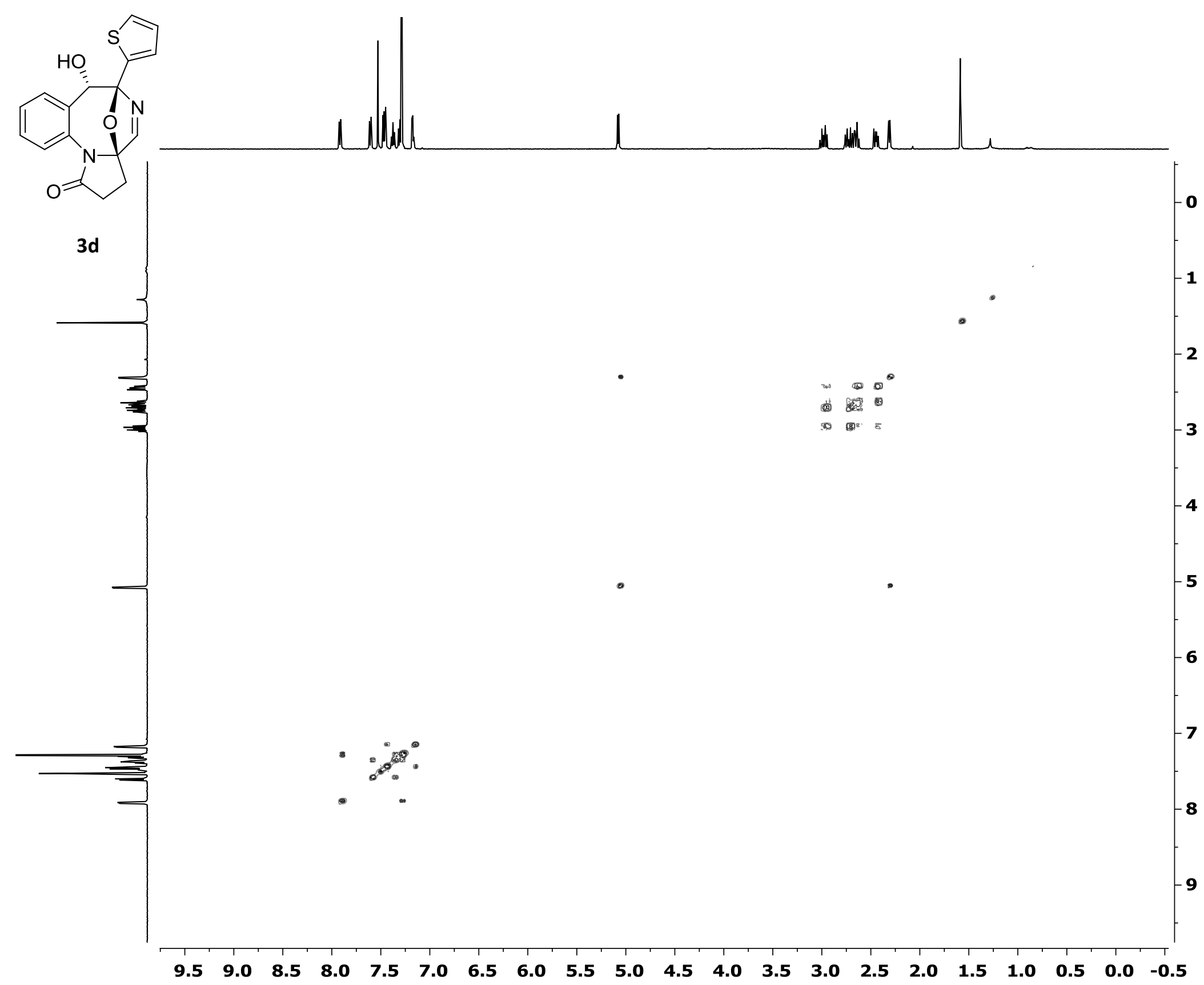




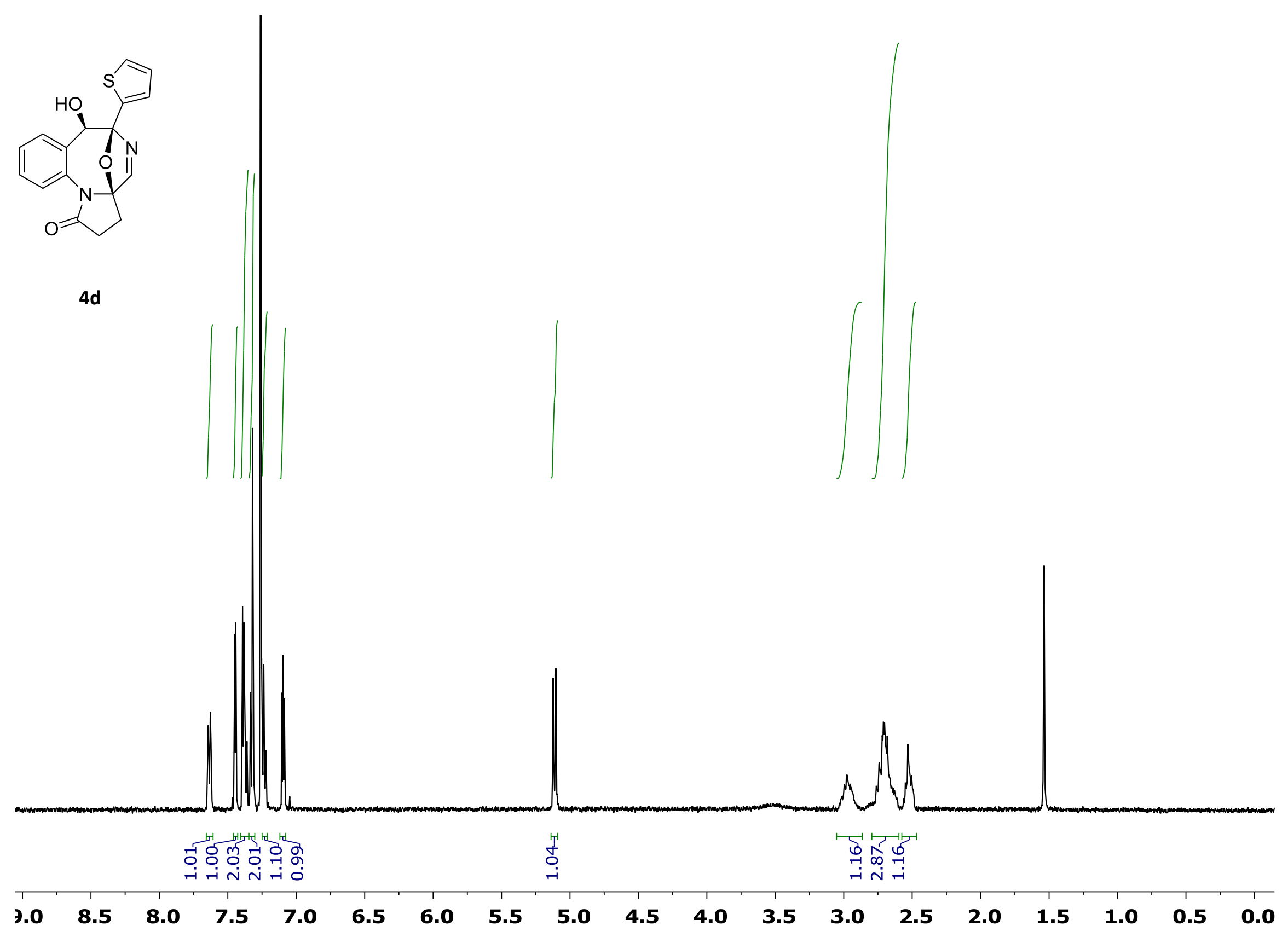




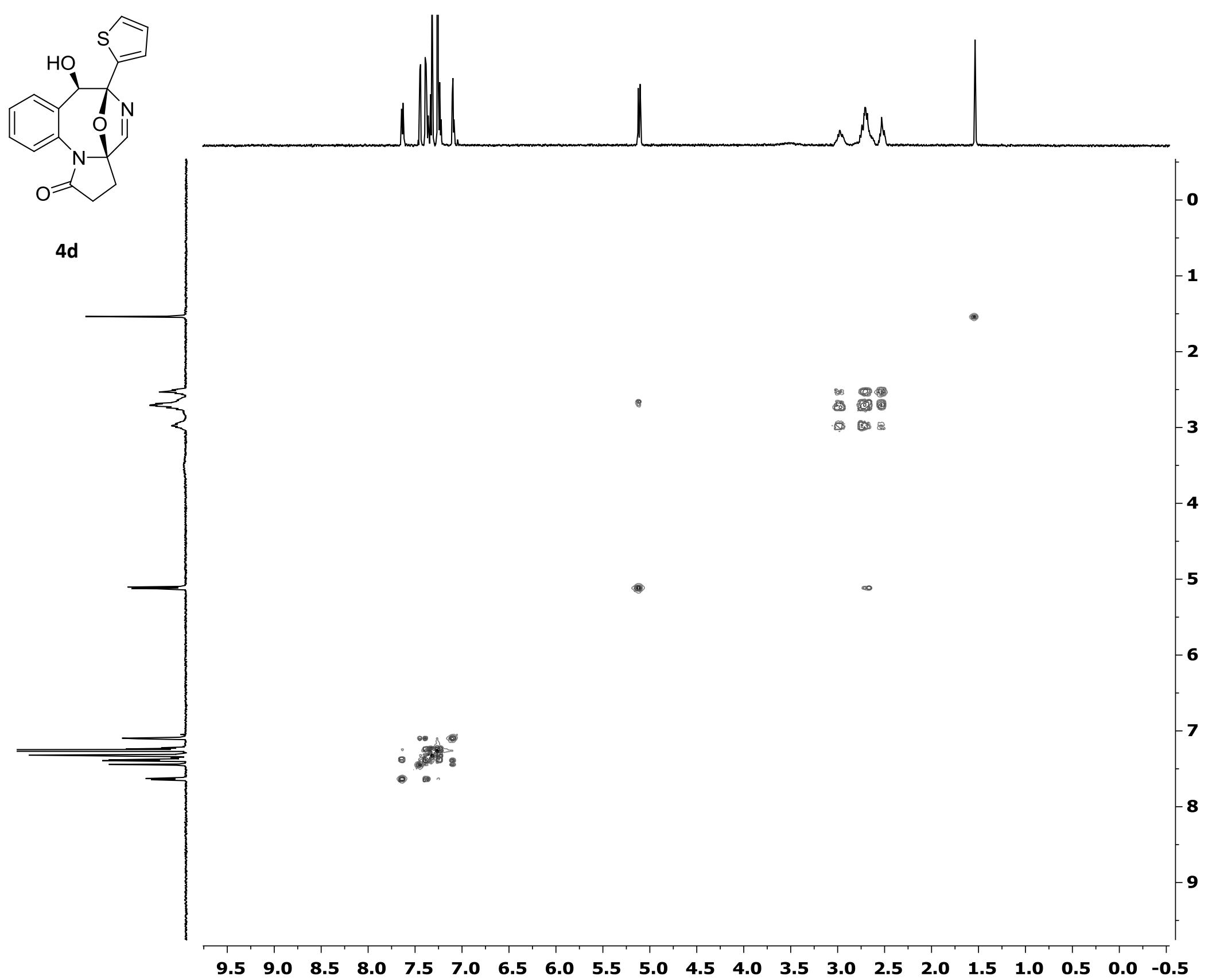



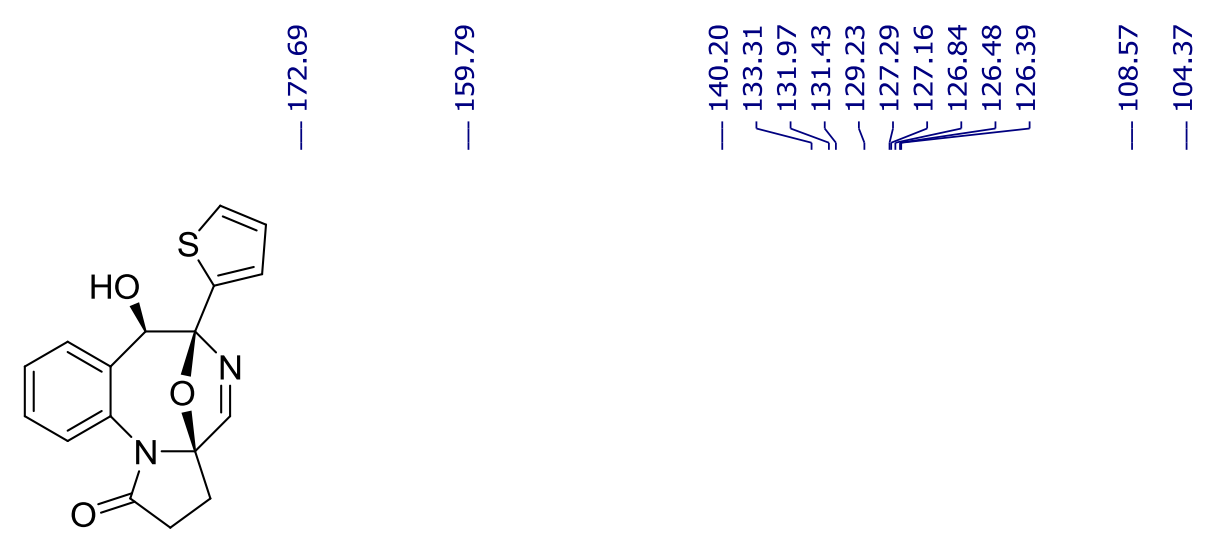

$\stackrel{0}{\substack{\infty \\ \infty \\ 1}}$

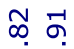

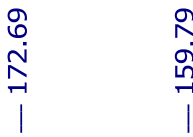

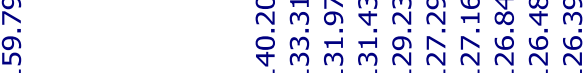

|

|

sin

$4 d$

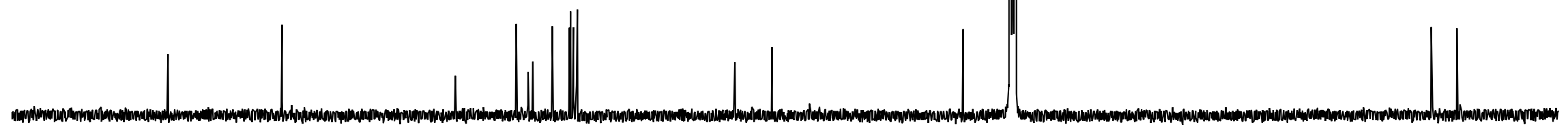

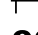

170

160

150

140

130

120

110

100

90

80

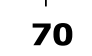

60

50

40

30

20 
<smiles>O=C(N[C@H]1[C@@H](O)c2ccccc2N2C(=O)CC[C@@]12O)c1cccs1</smiles>
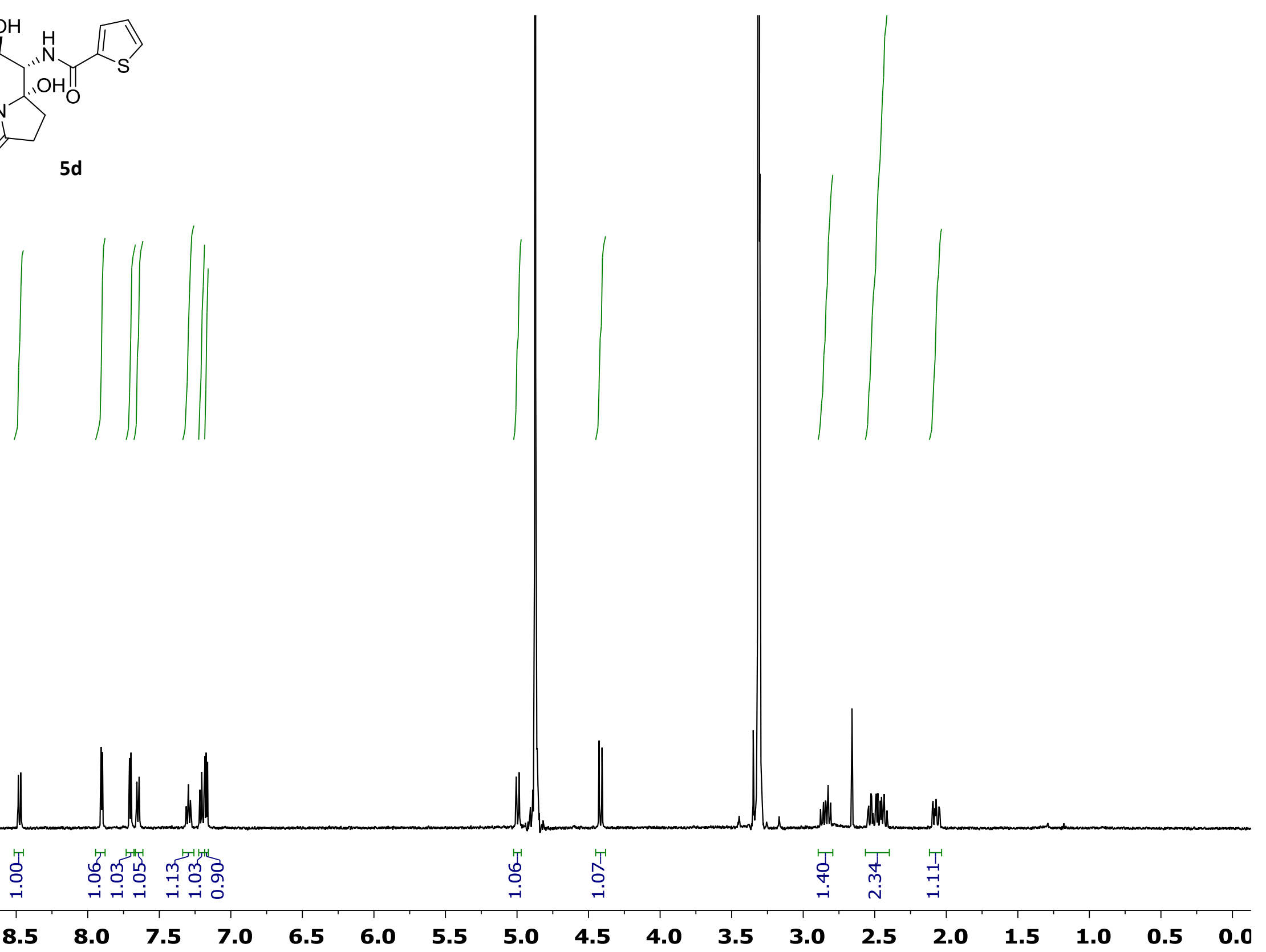
\begin{tabular}{ll}
+ & 0 \\
+ & 0 \\
\multirow{1}{*}{} & 0 \\
\hline &
\end{tabular}

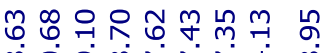

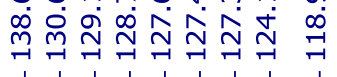

|

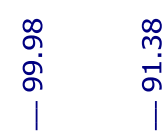

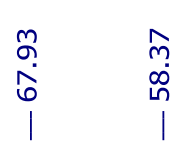

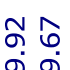

จิ

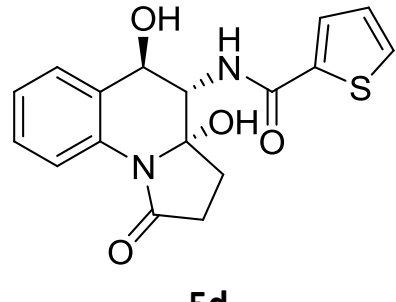

$5 d$

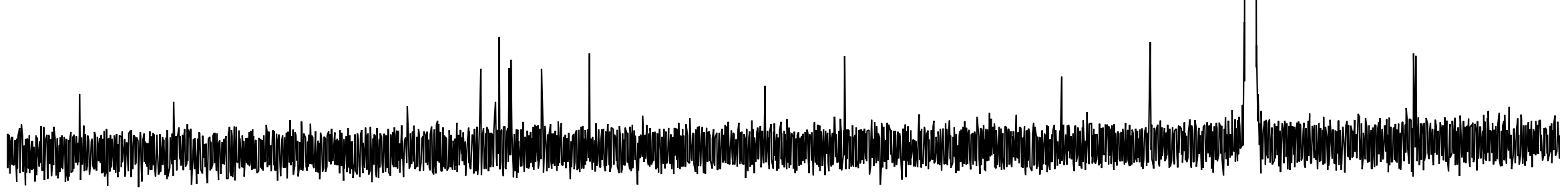


Photoassisted Diversity-Oriented Synthesis: Intramolecular Cycloadditions...

N.N. Bhuvan Kumar and Andrei G. Kutateladze, S106

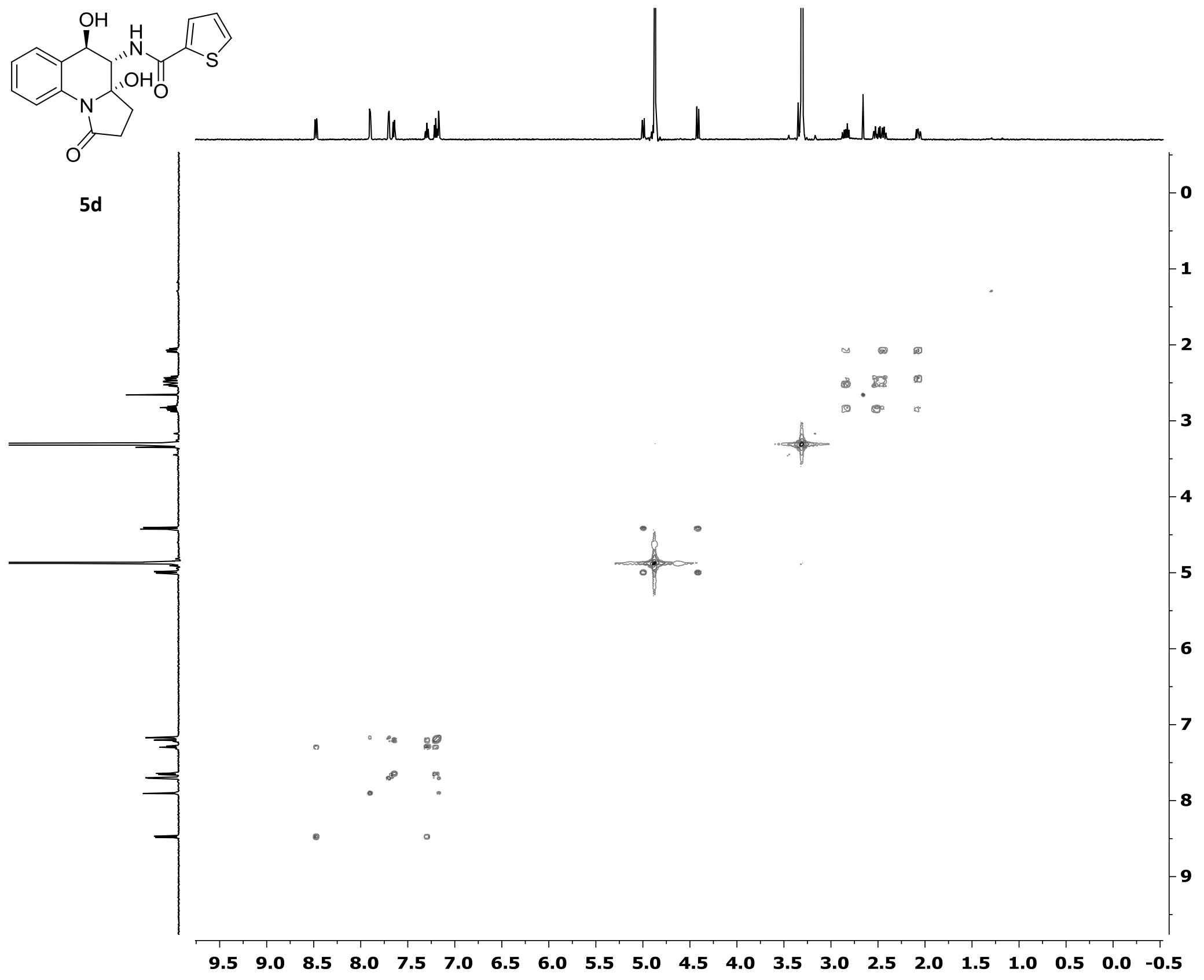


Photoassisted Diversity-Oriented Synthesis: Intramolecular Cycloadditions...

N.N. Bhuvan Kumar and Andrei G. Kutateladze, S107

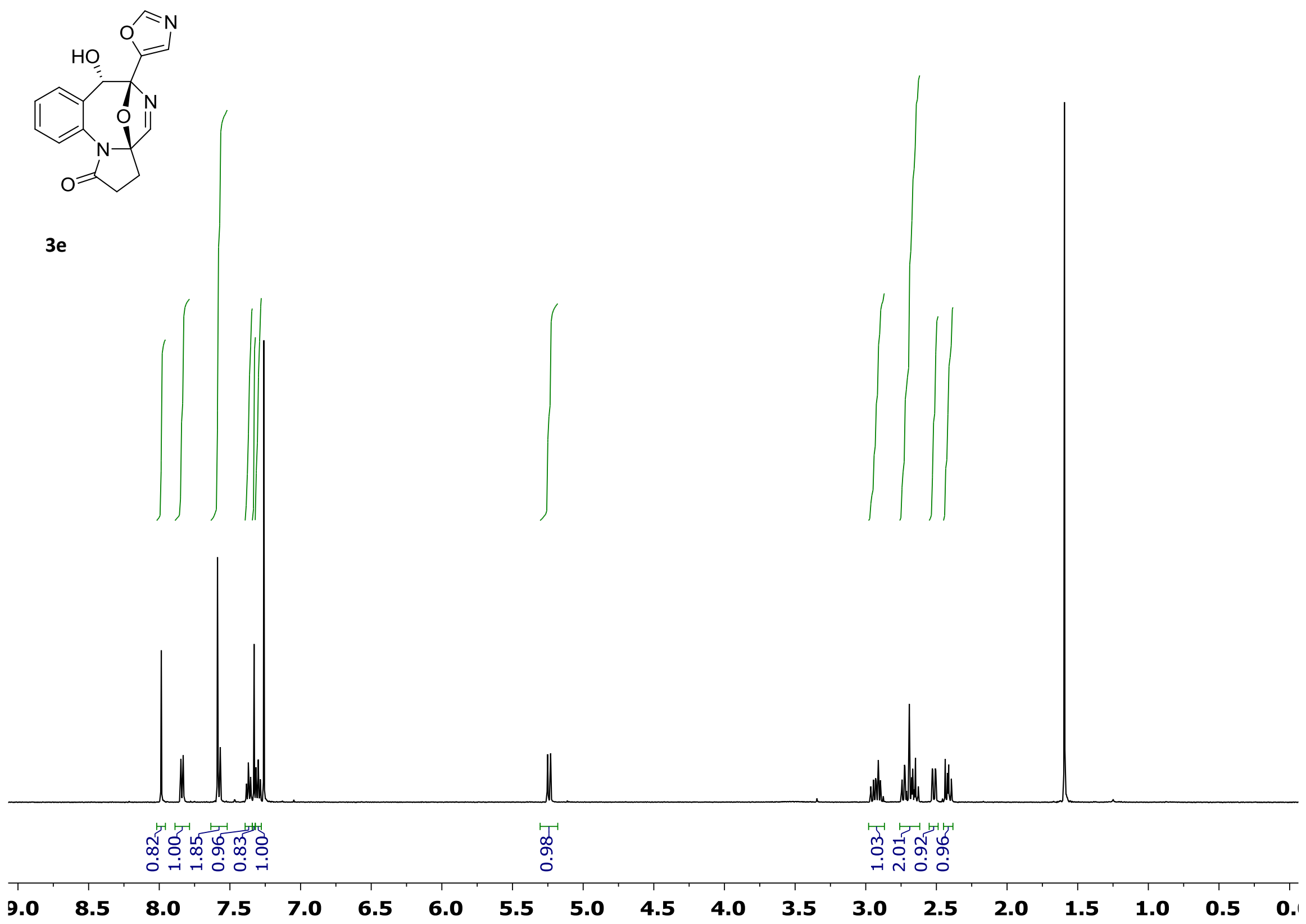



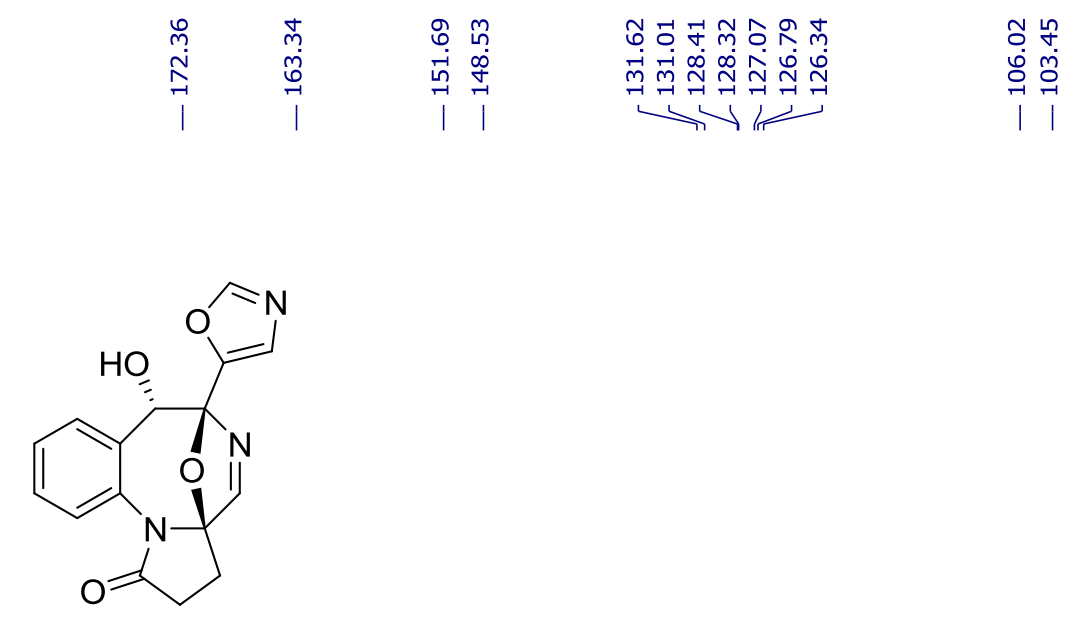

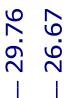

$3 e$

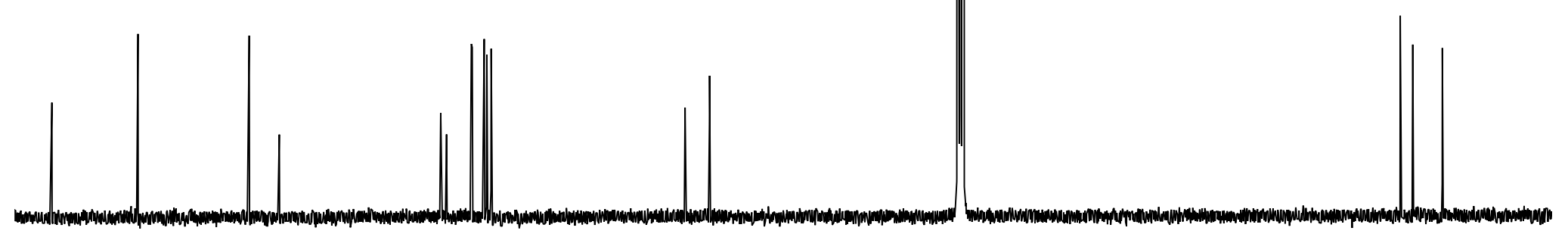

$\begin{array}{lll}170 & 160 & 150\end{array}$

140130

120110

100

90

80

7060

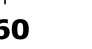

50

40

30 


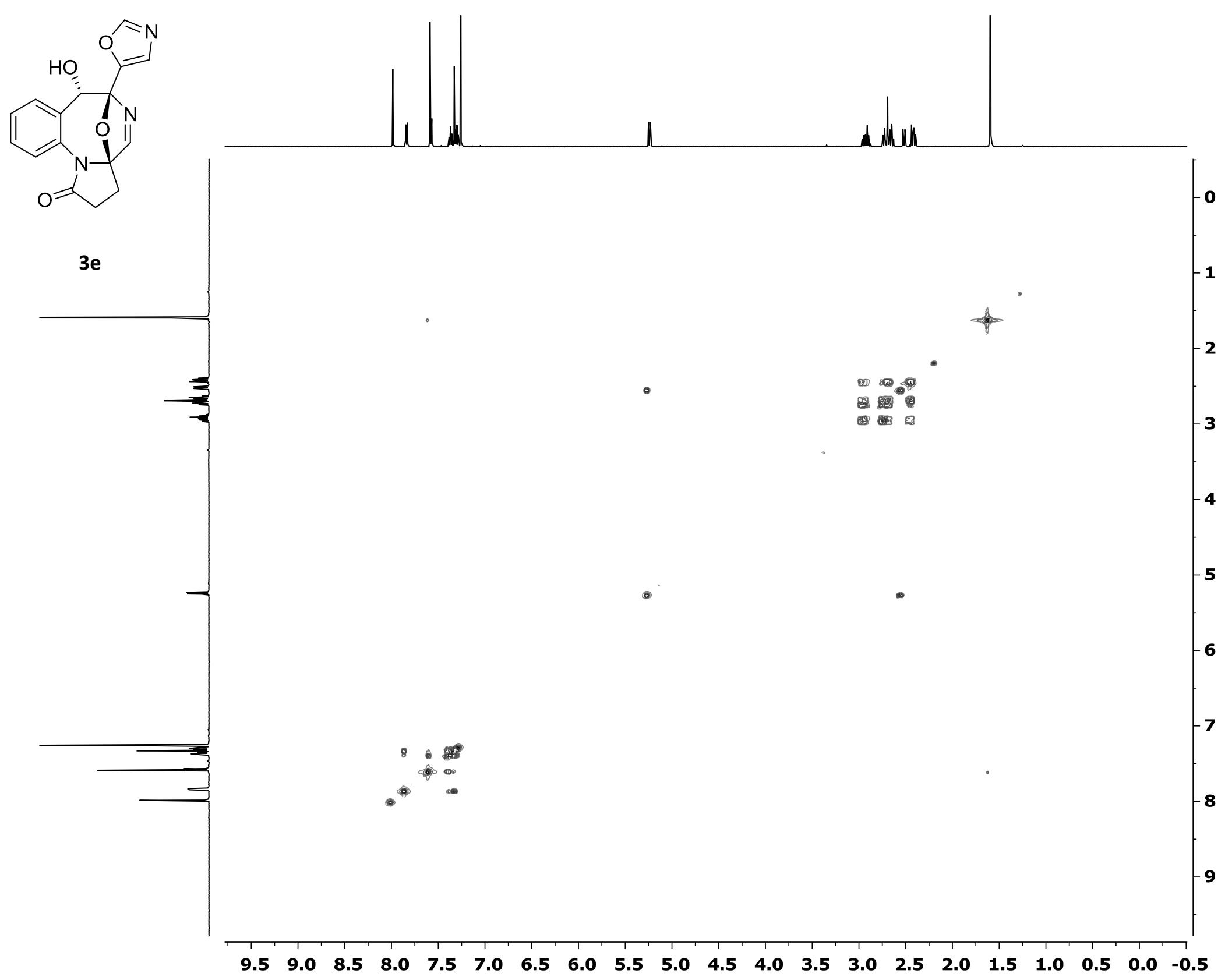



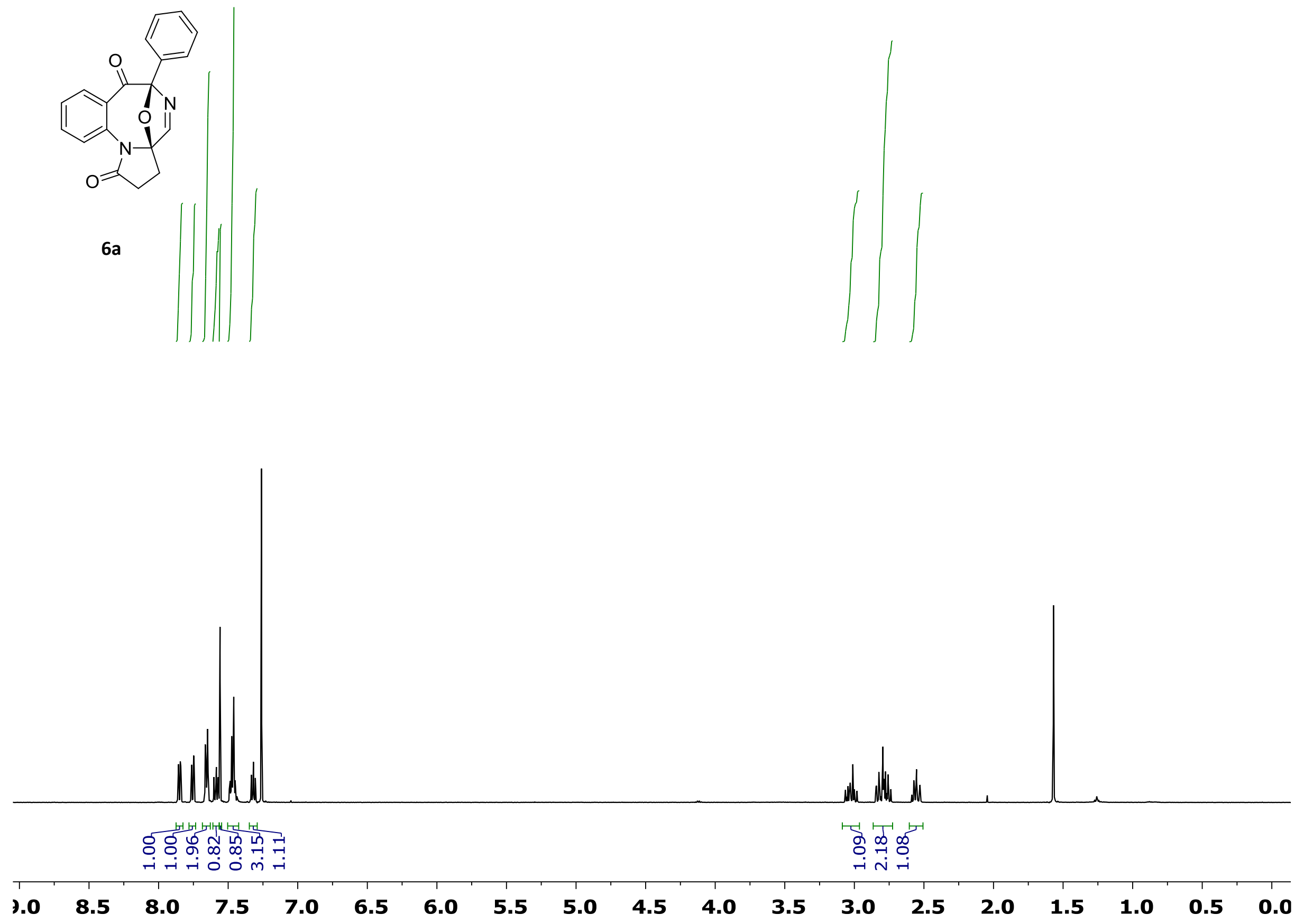
Photoassisted Diversity-Oriented Synthesis: Intramolecular Cycloadditions...

N.N. Bhuvan Kumar and Andrei G. Kutateladze, S111

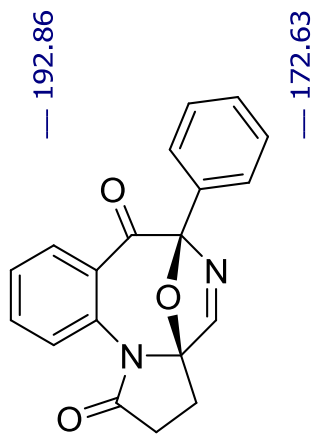

$6 a$
+
$\dot{0}$
-1
1

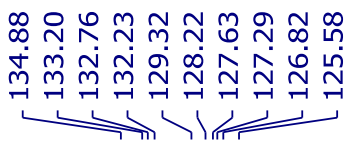

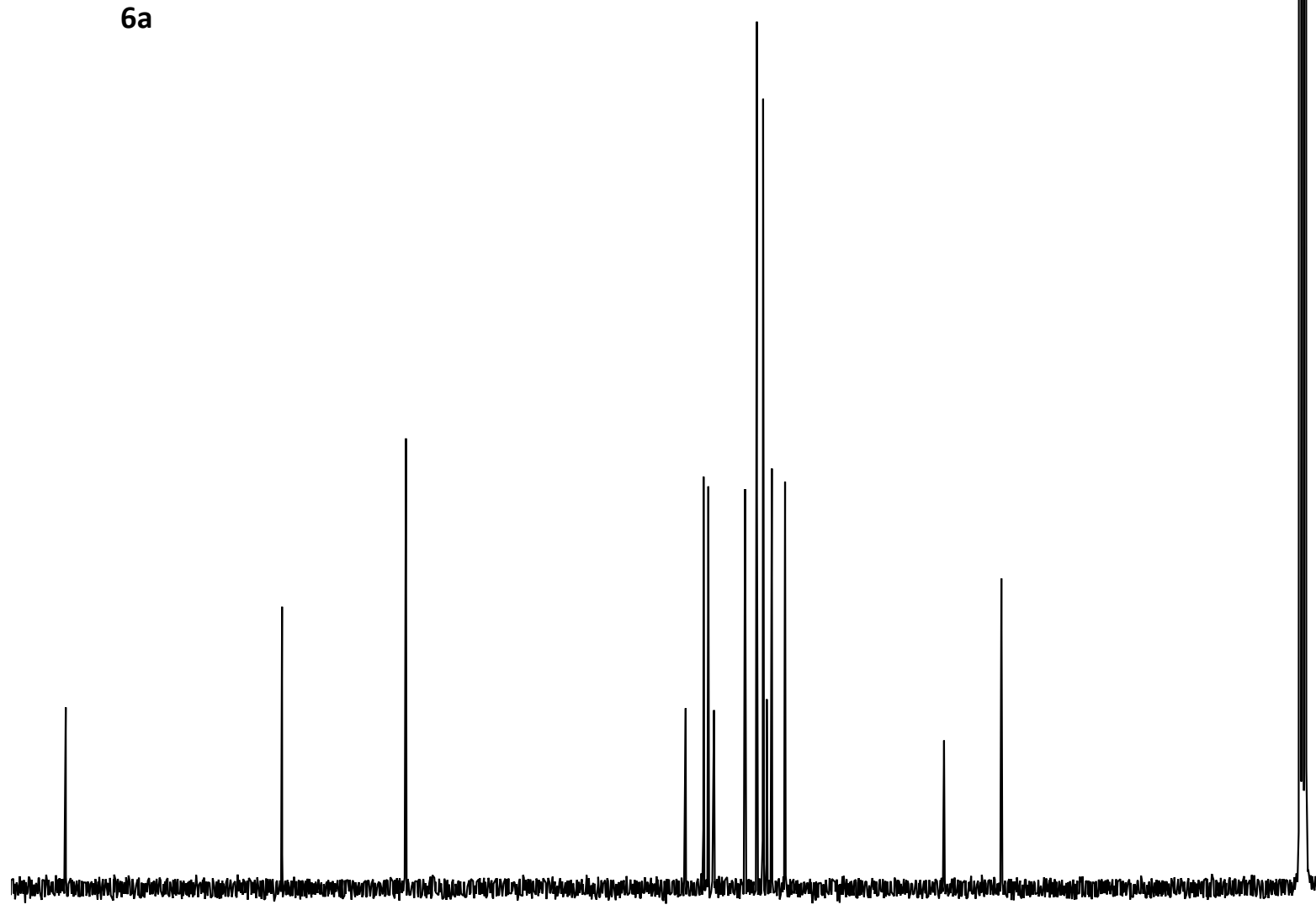
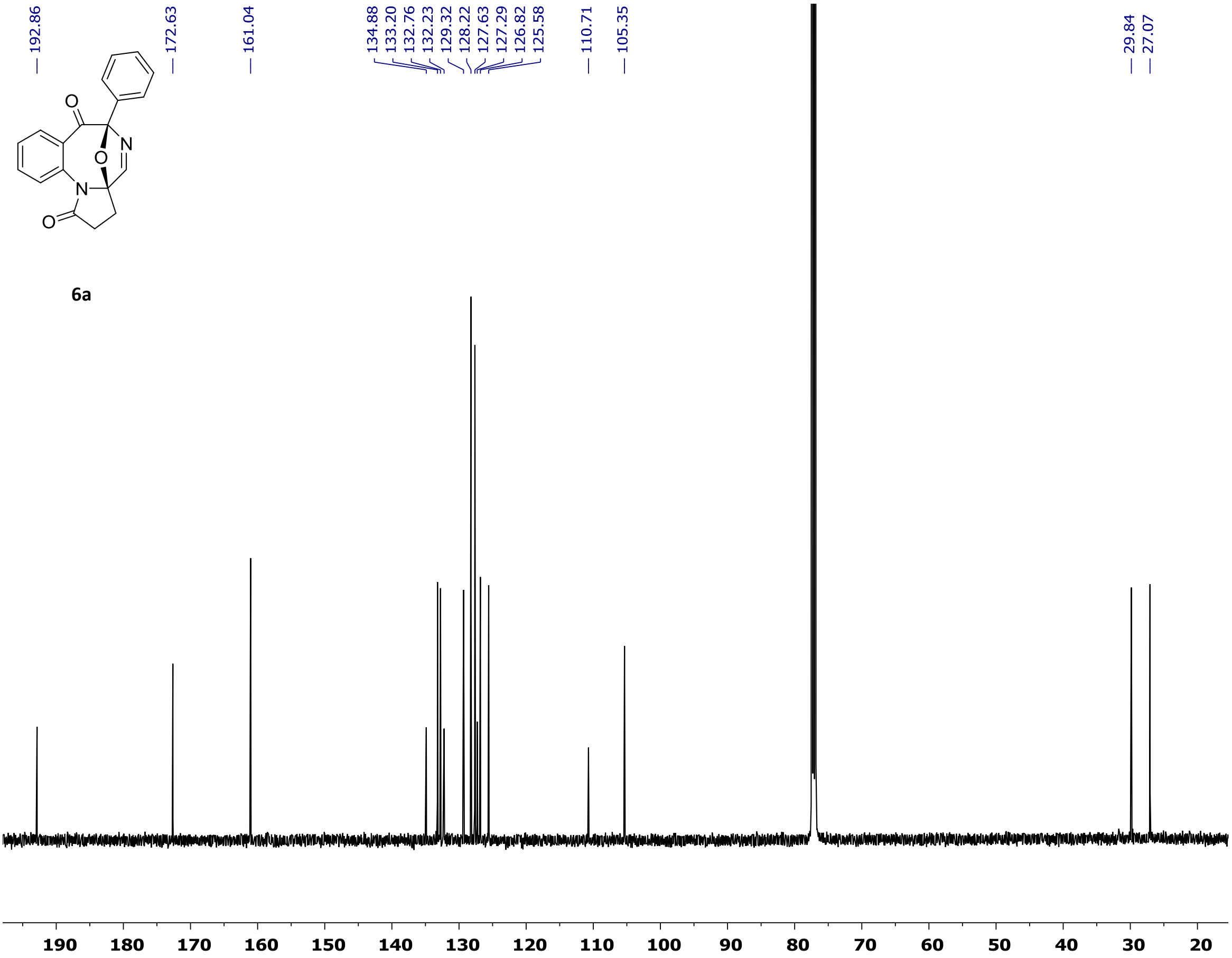


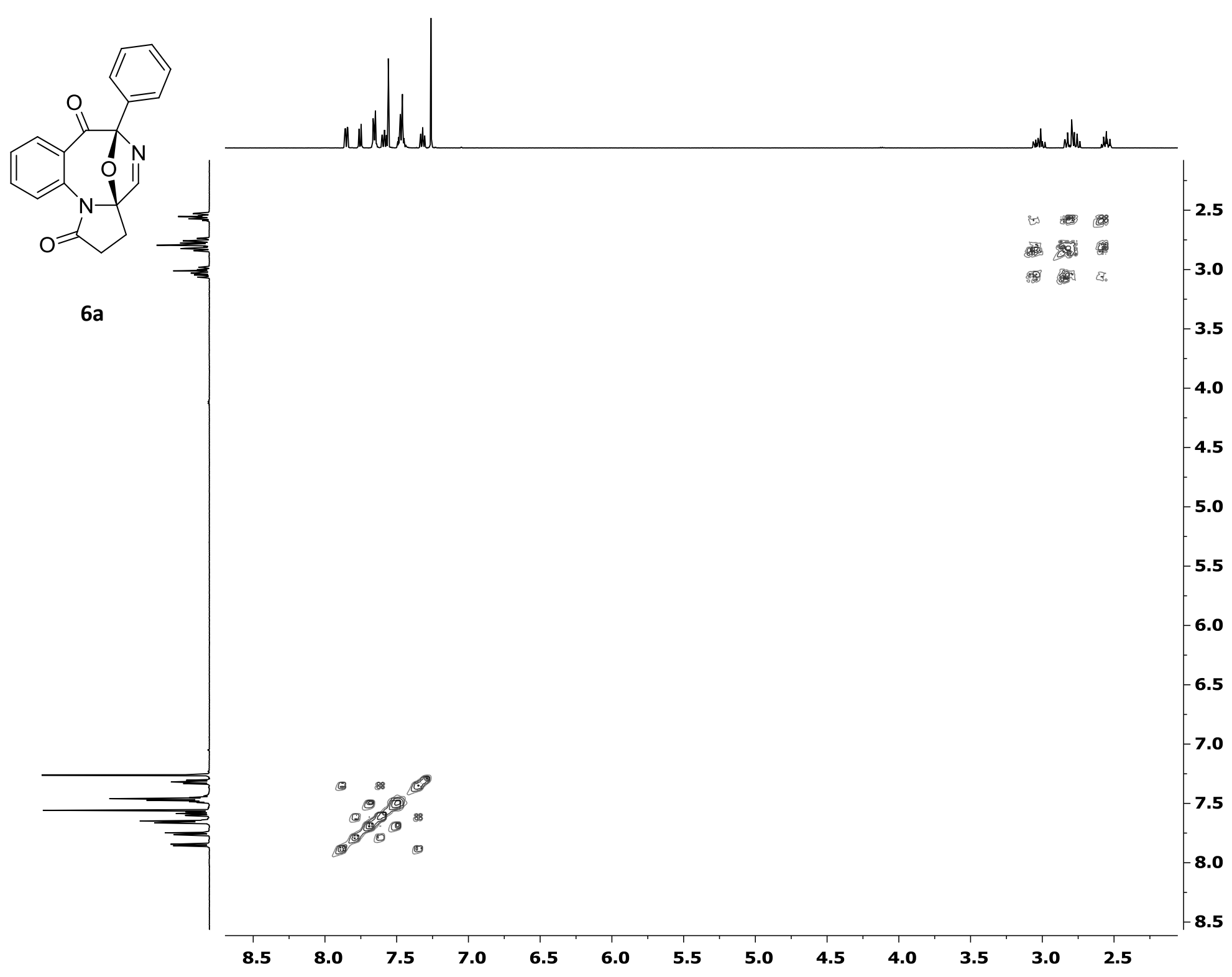




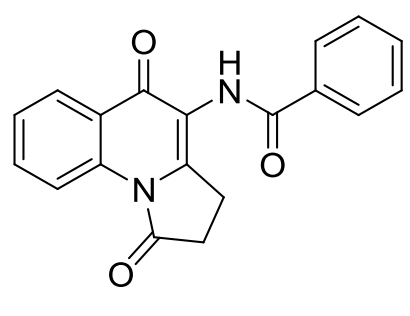

$7 a$
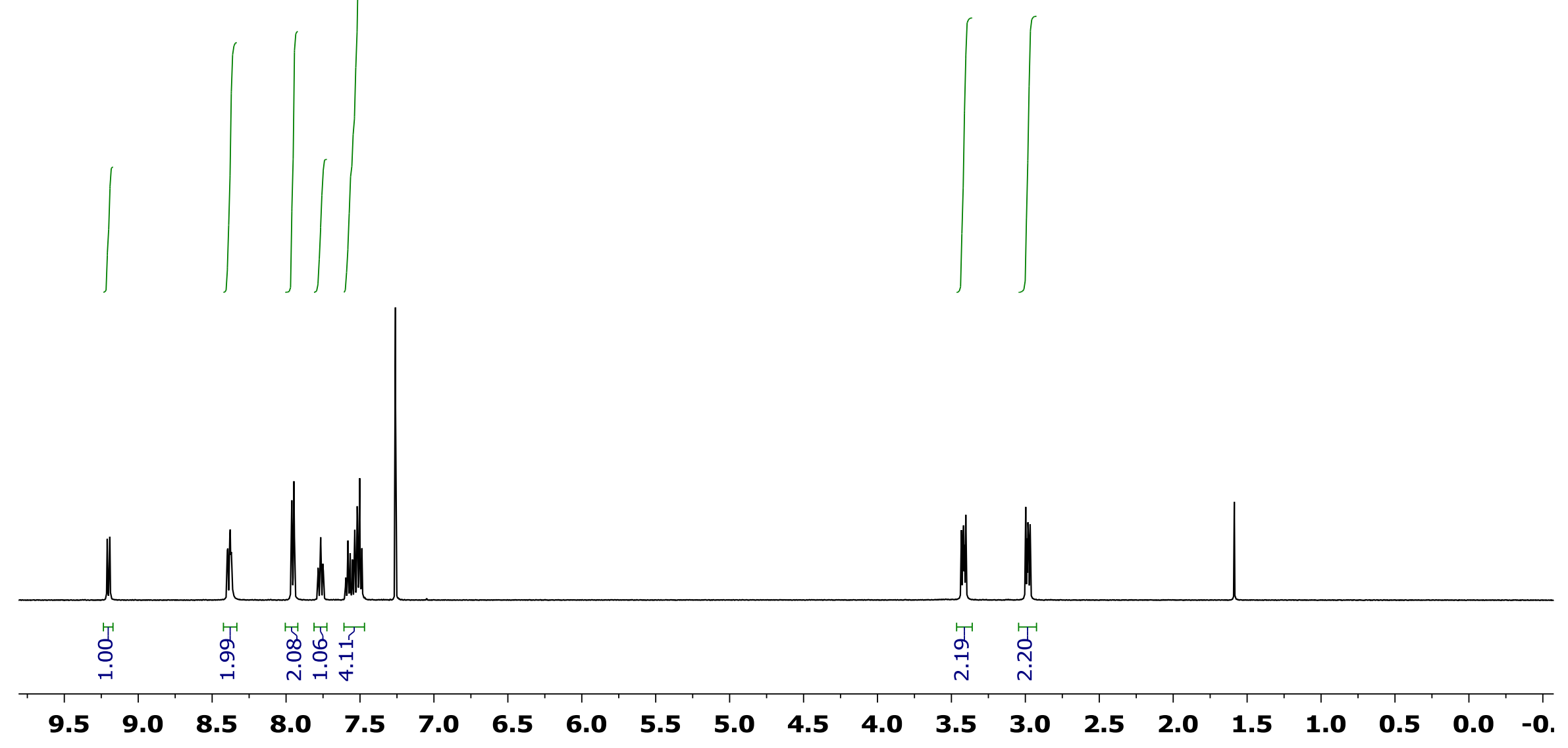

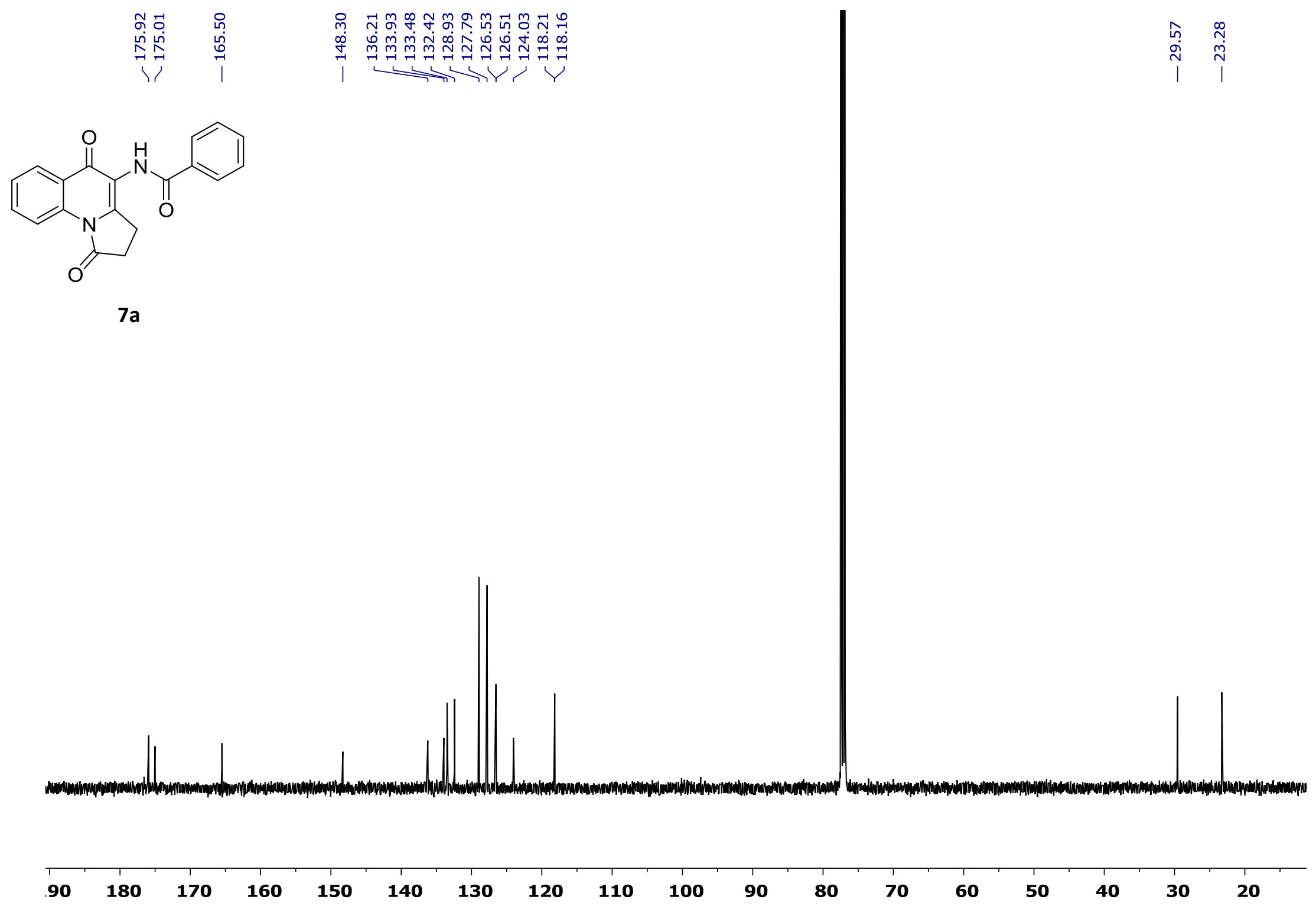


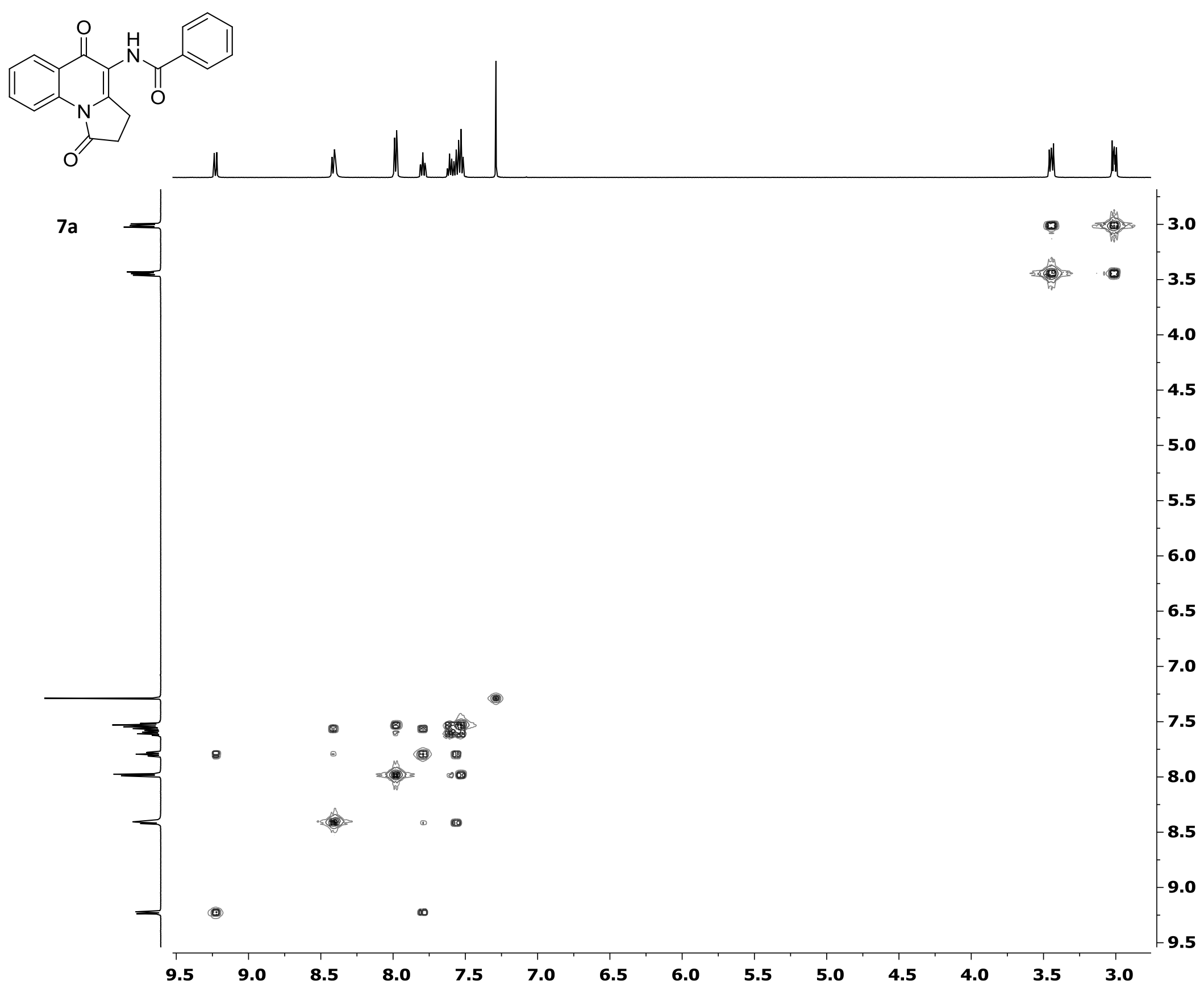




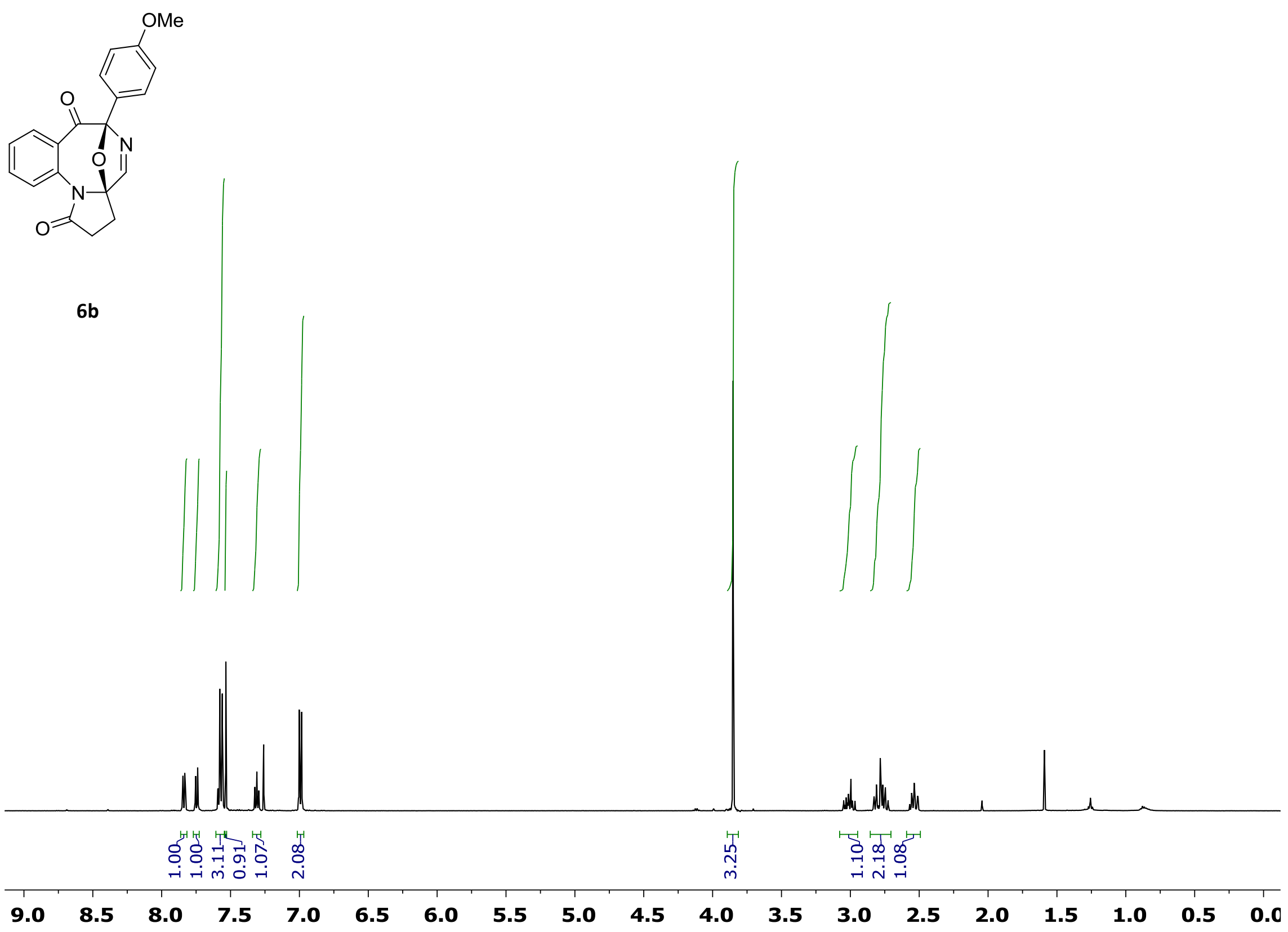




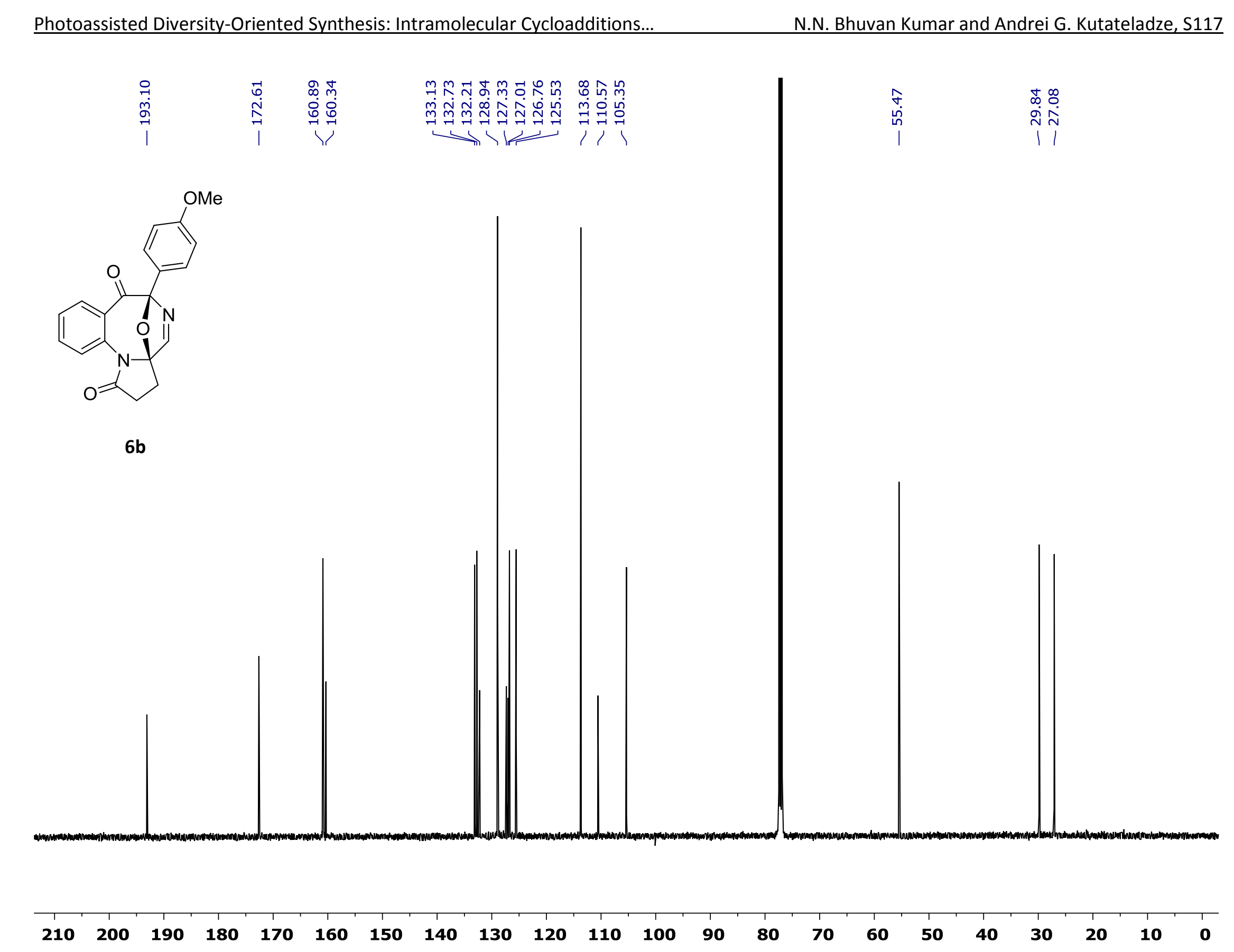




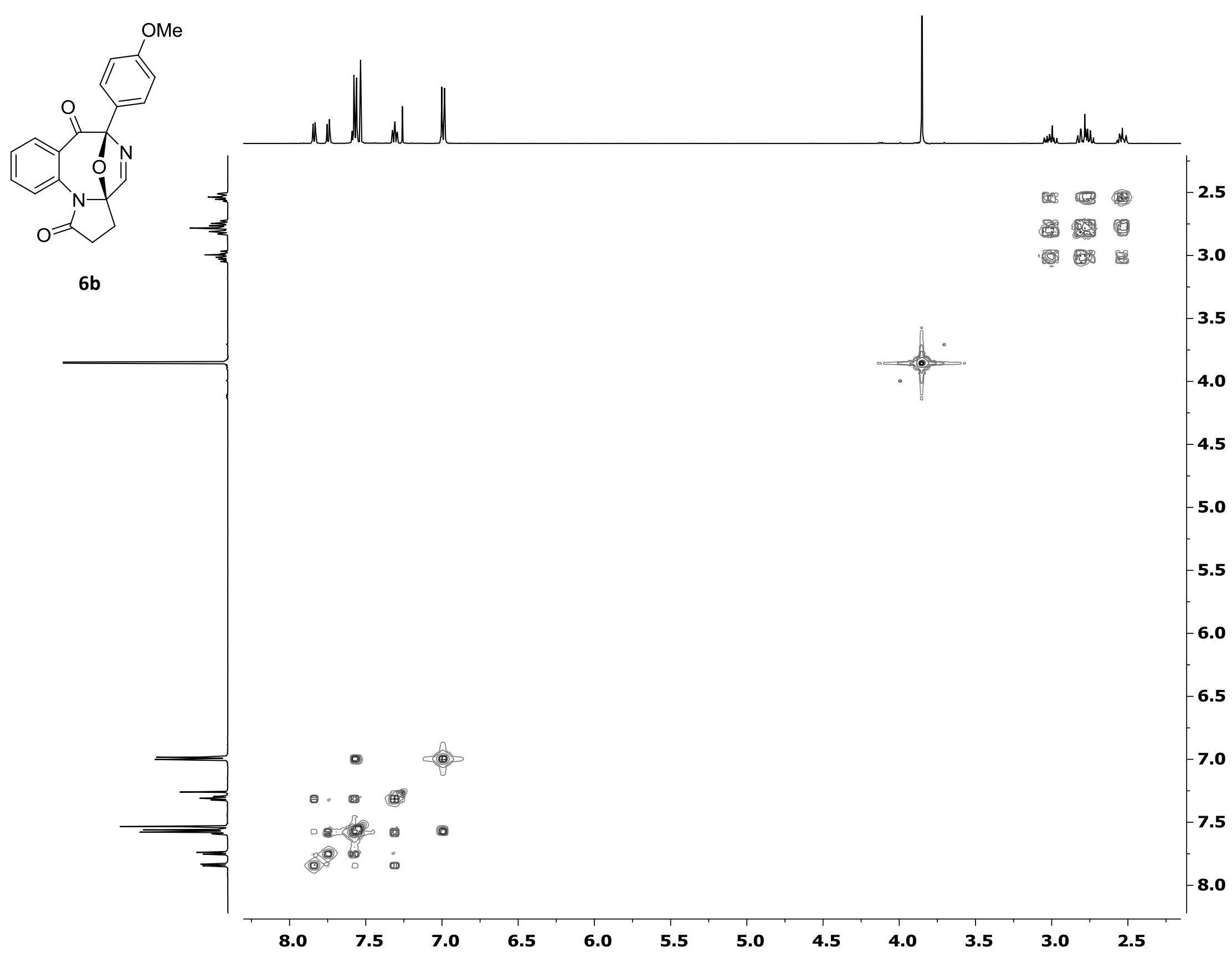




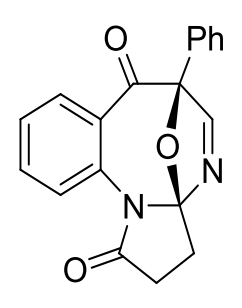

$11 f$
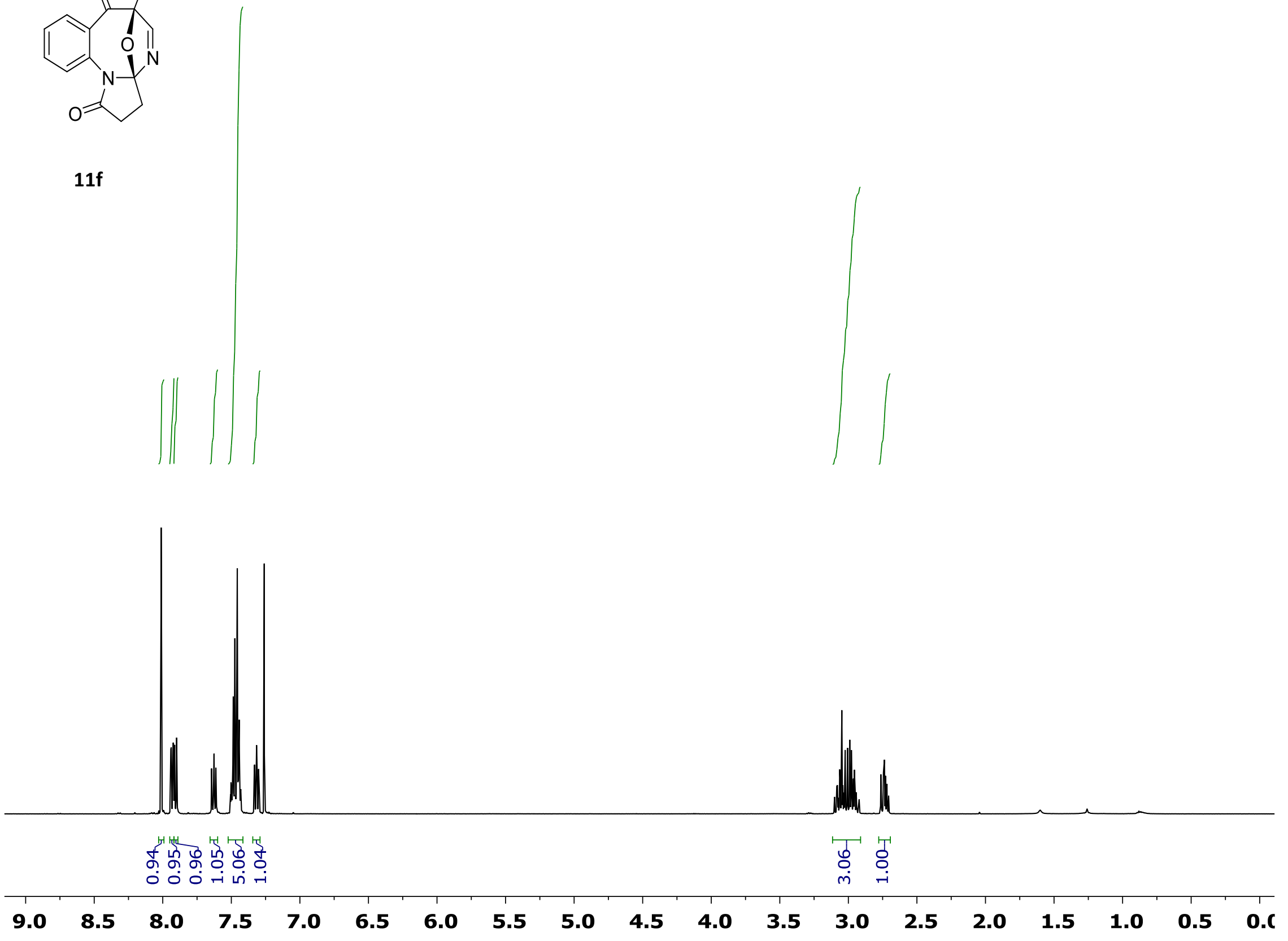

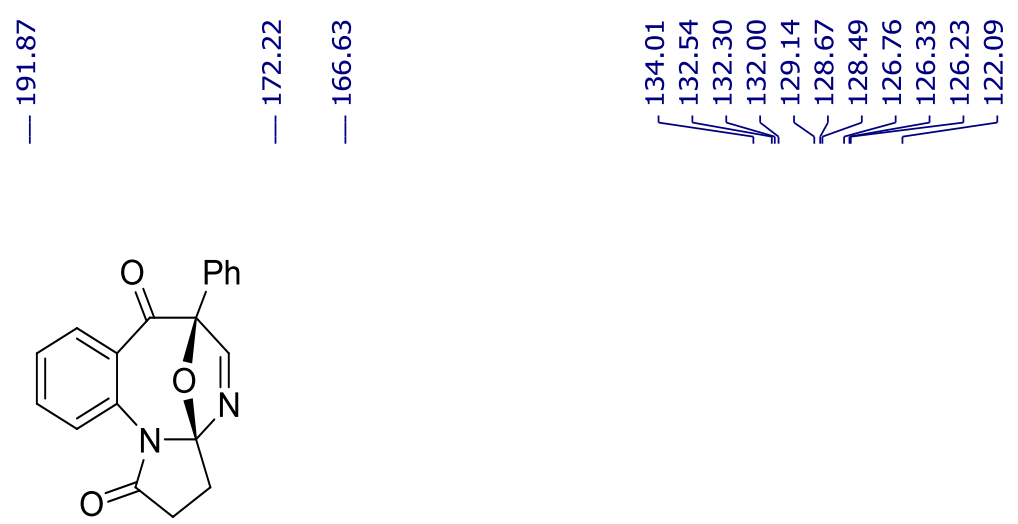

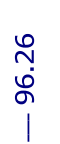

ำ

ก

$11 f$

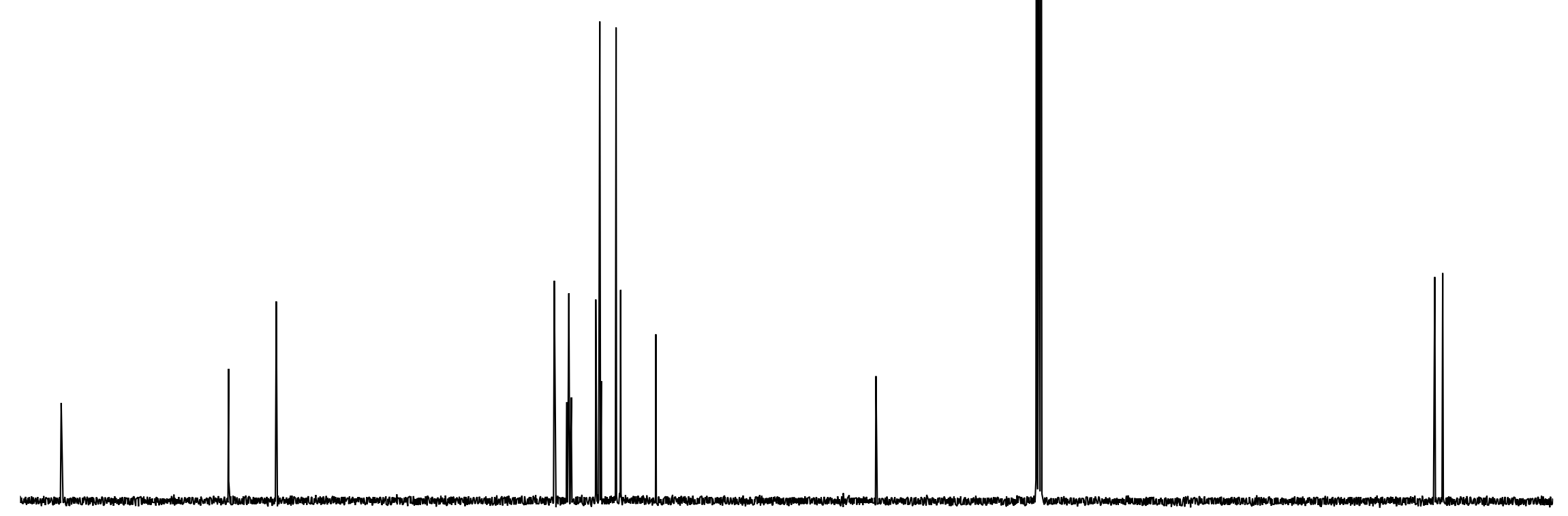




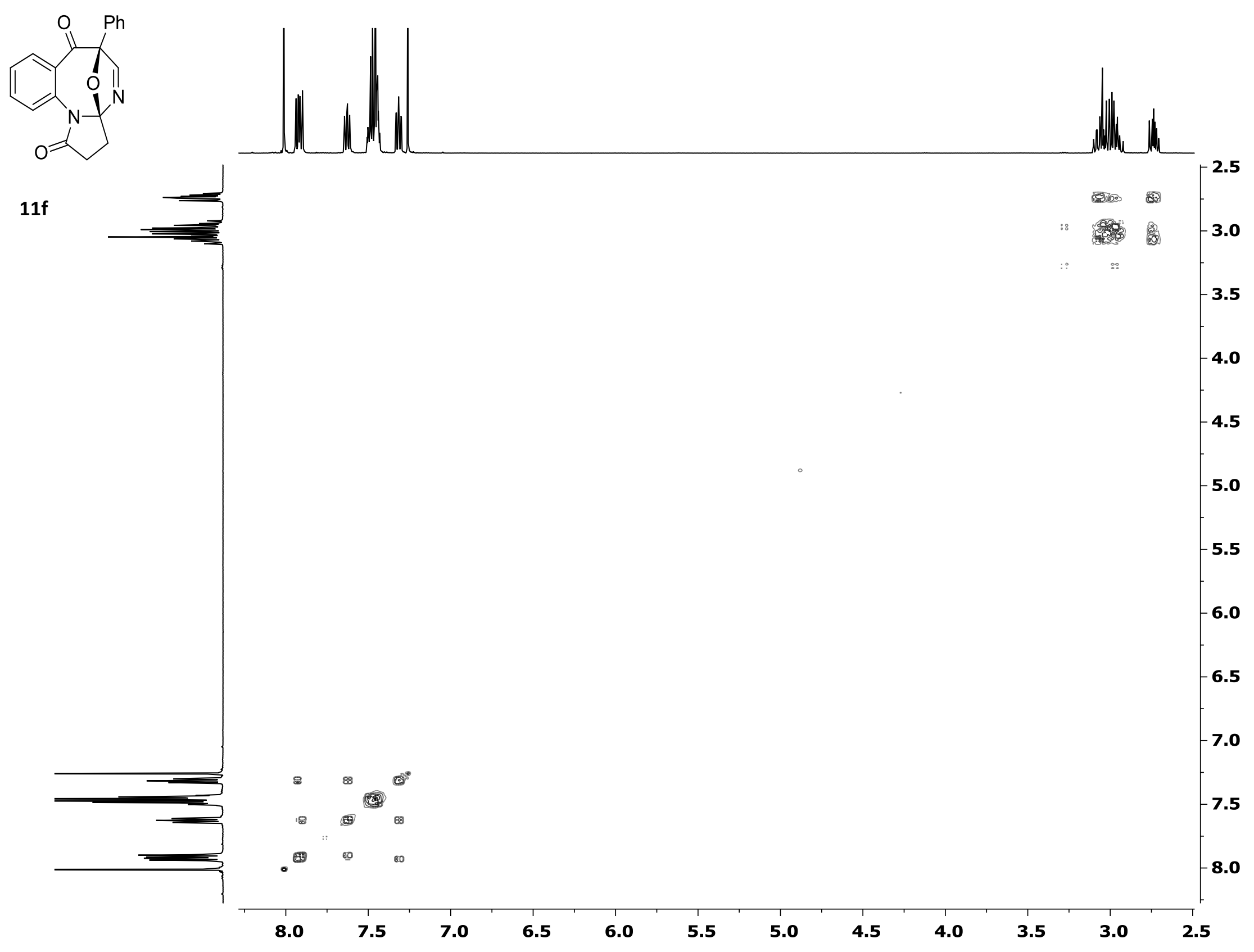



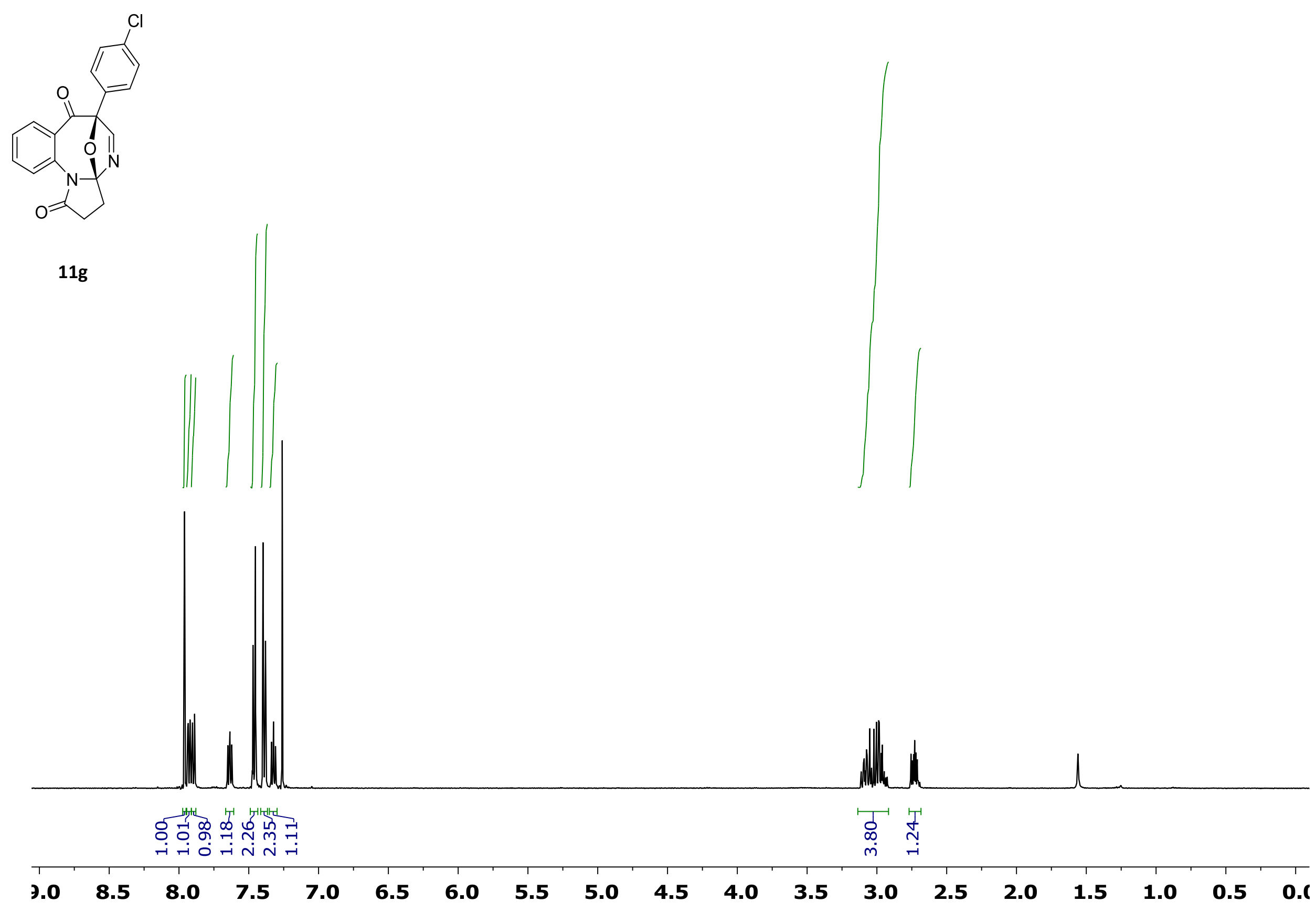


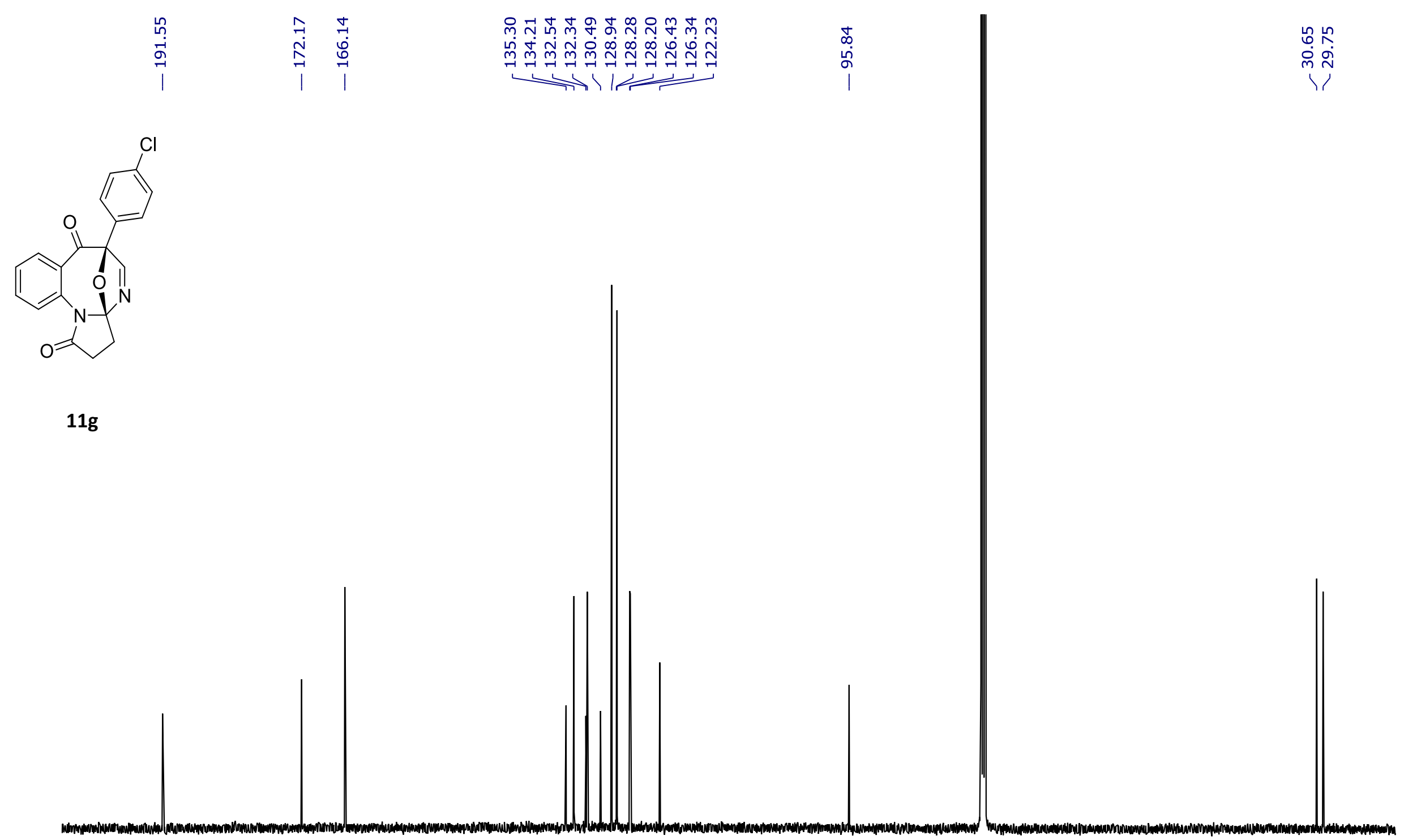
200
19018

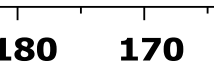
160
150
$140 \quad 130$
120
110
100
90
80

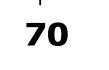
60
50
40
30 

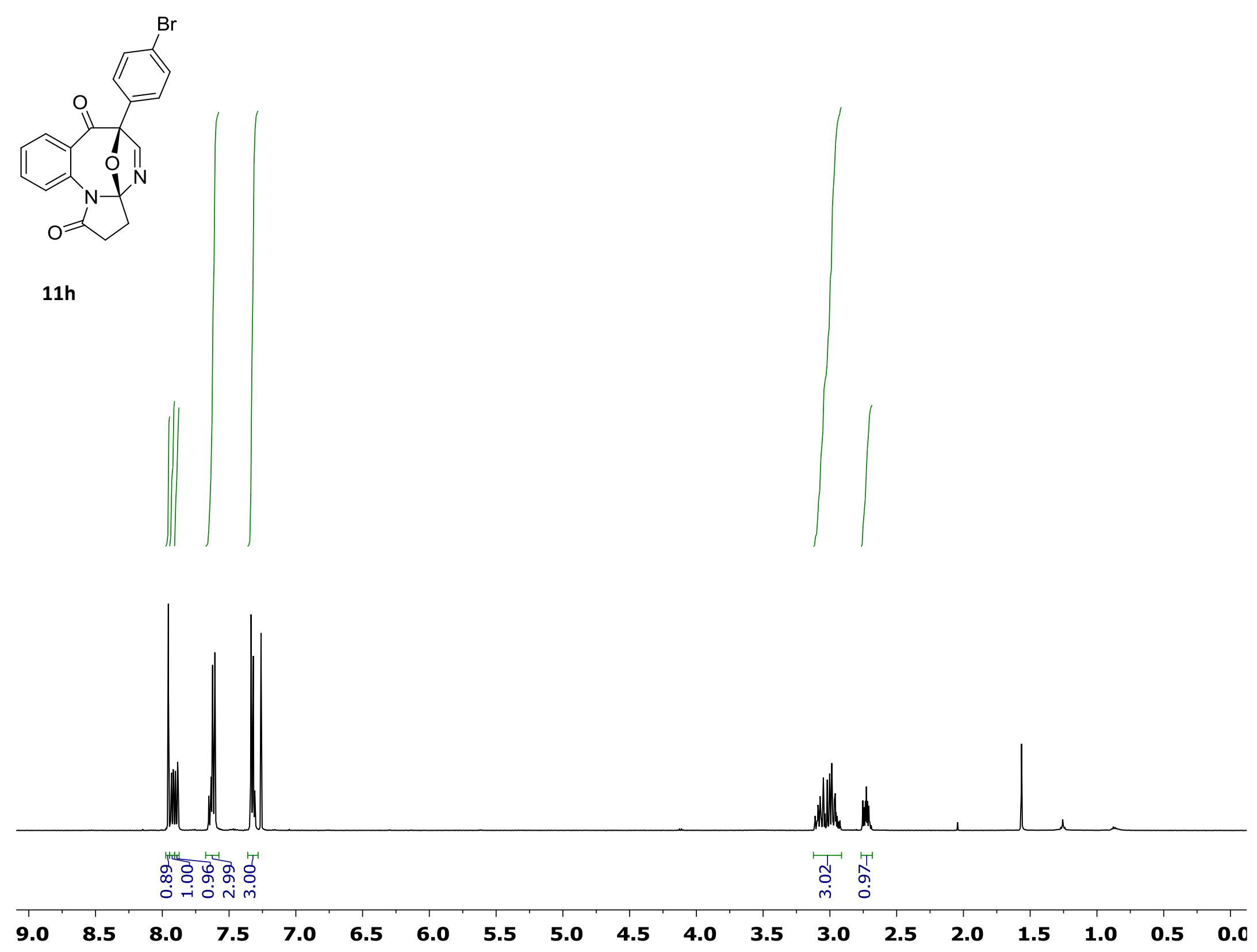


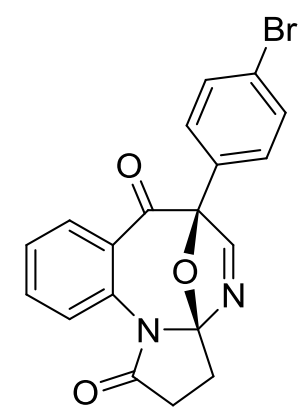

$11 \mathrm{~h}$

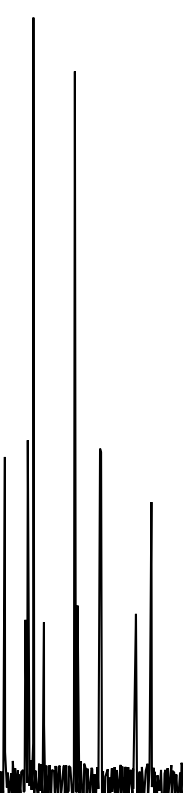




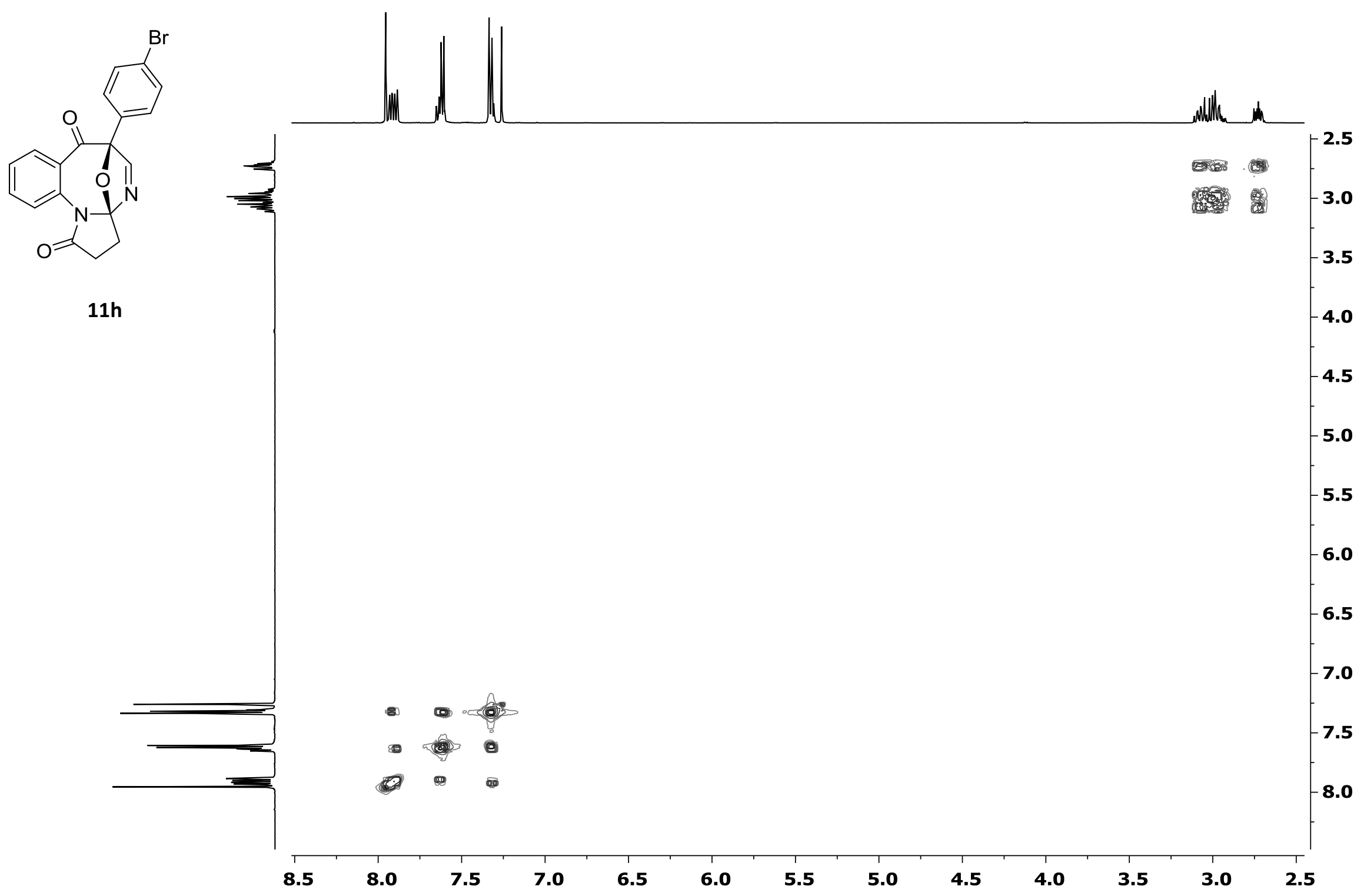




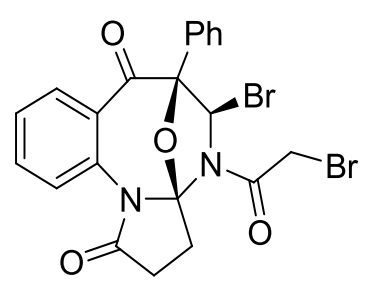

In situ preparation of dibromide $\mathbf{1 2}$ - NMR of reaction mixture

12

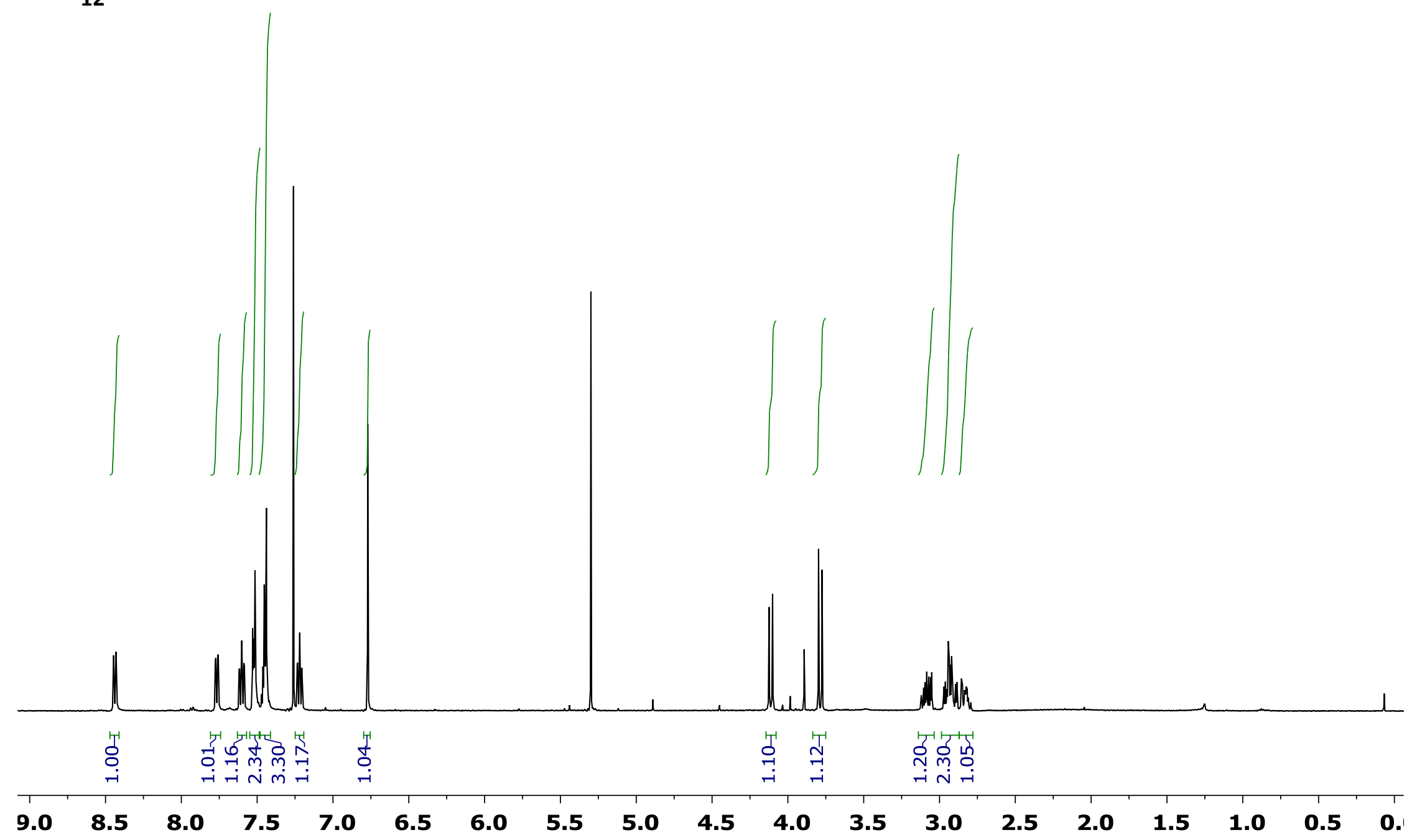




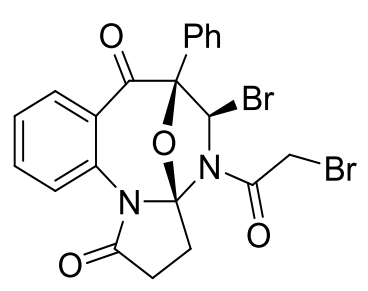

12

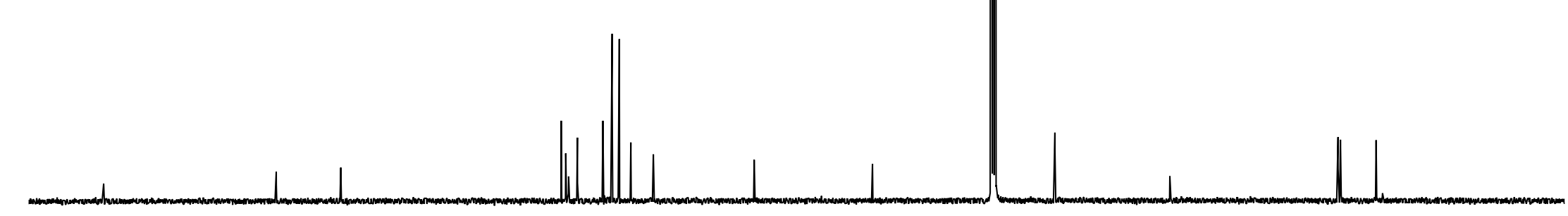




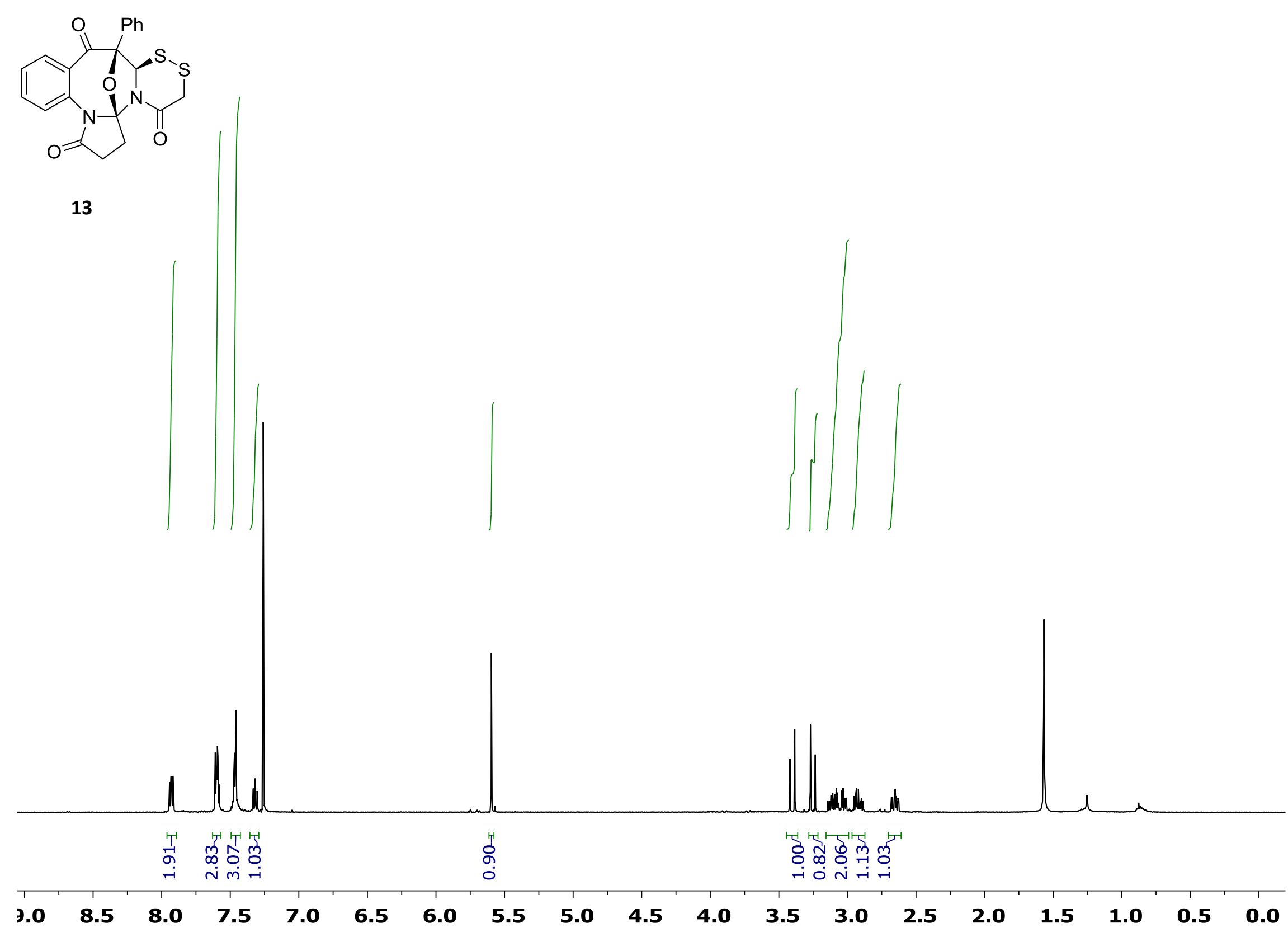



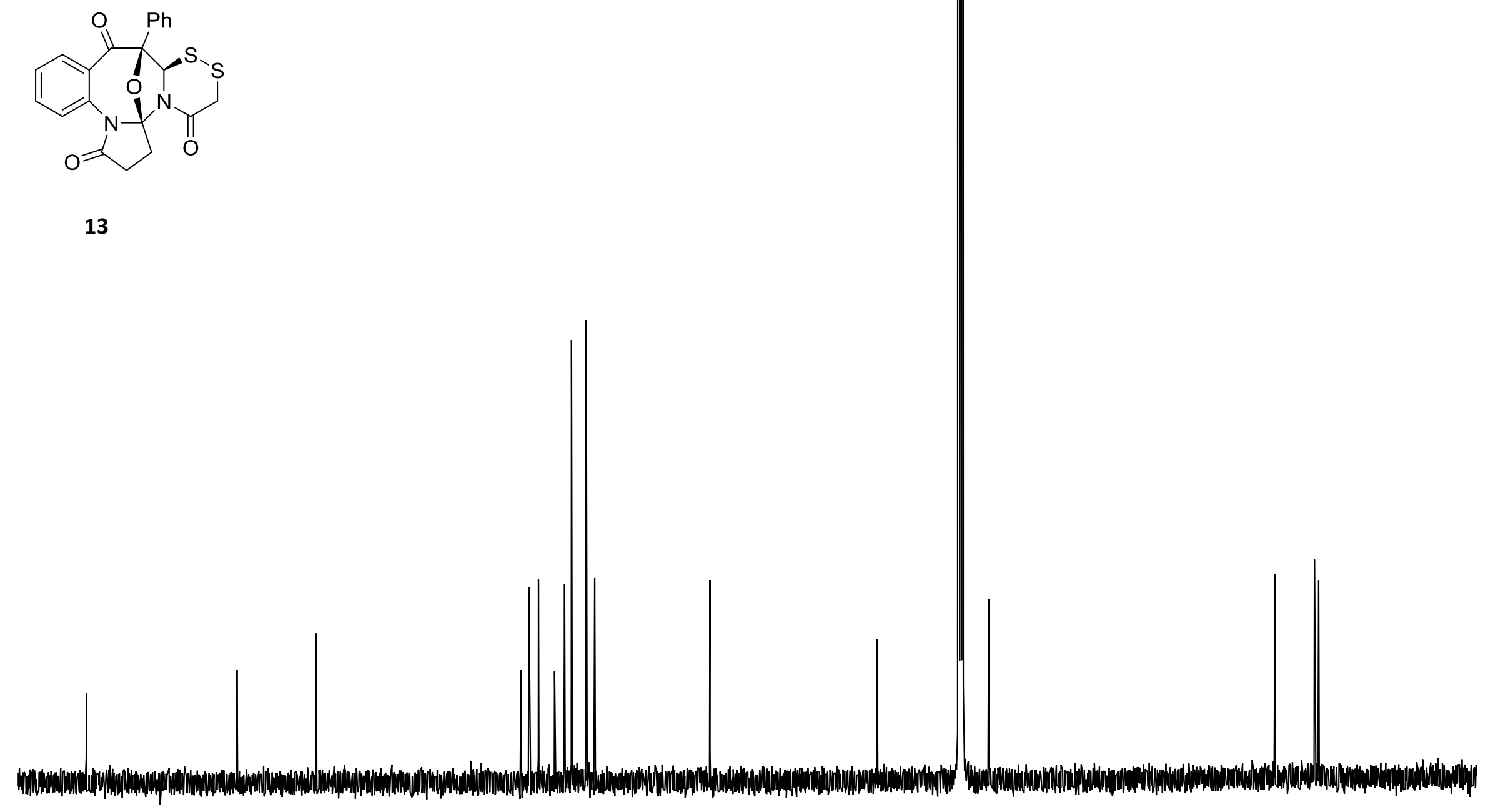


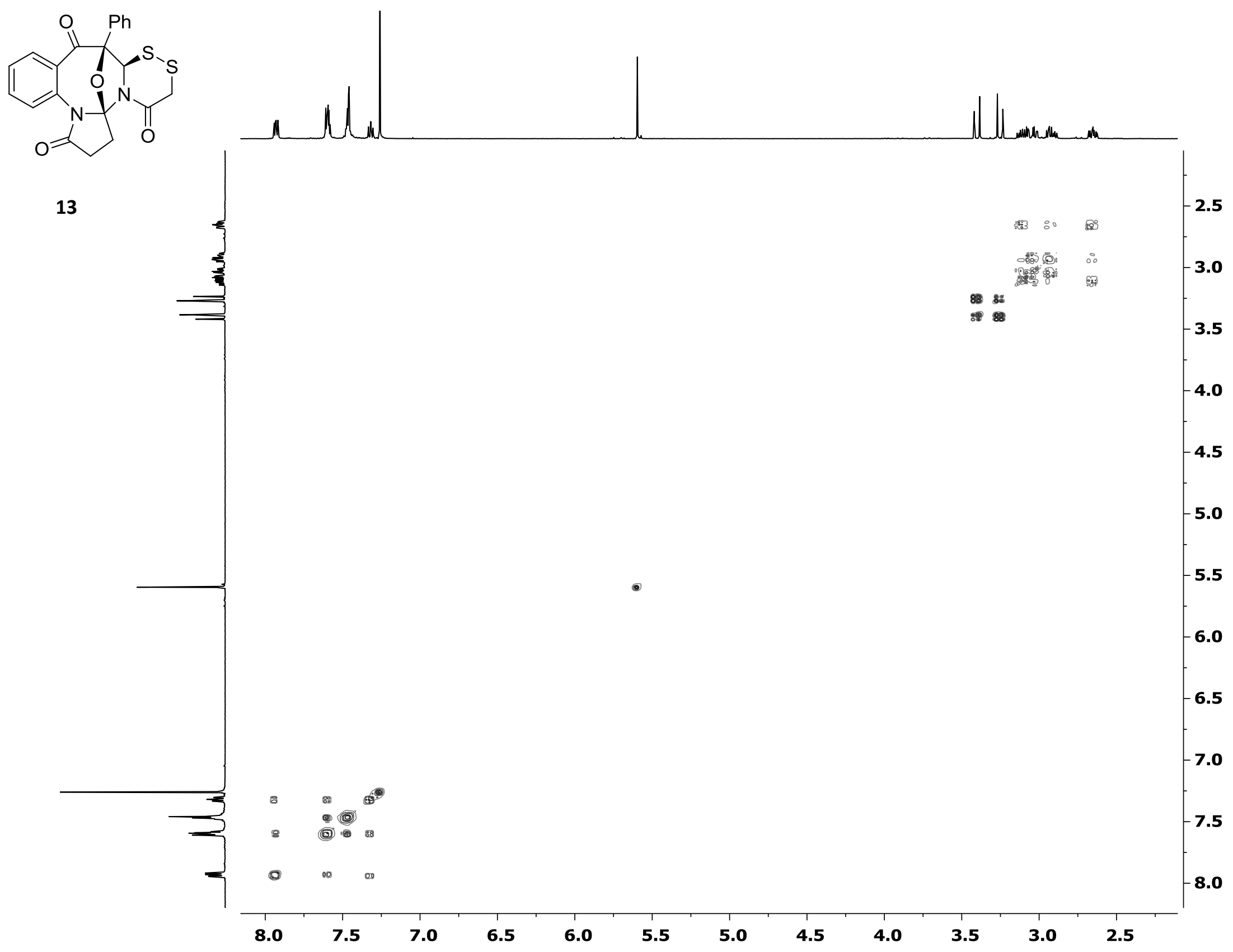



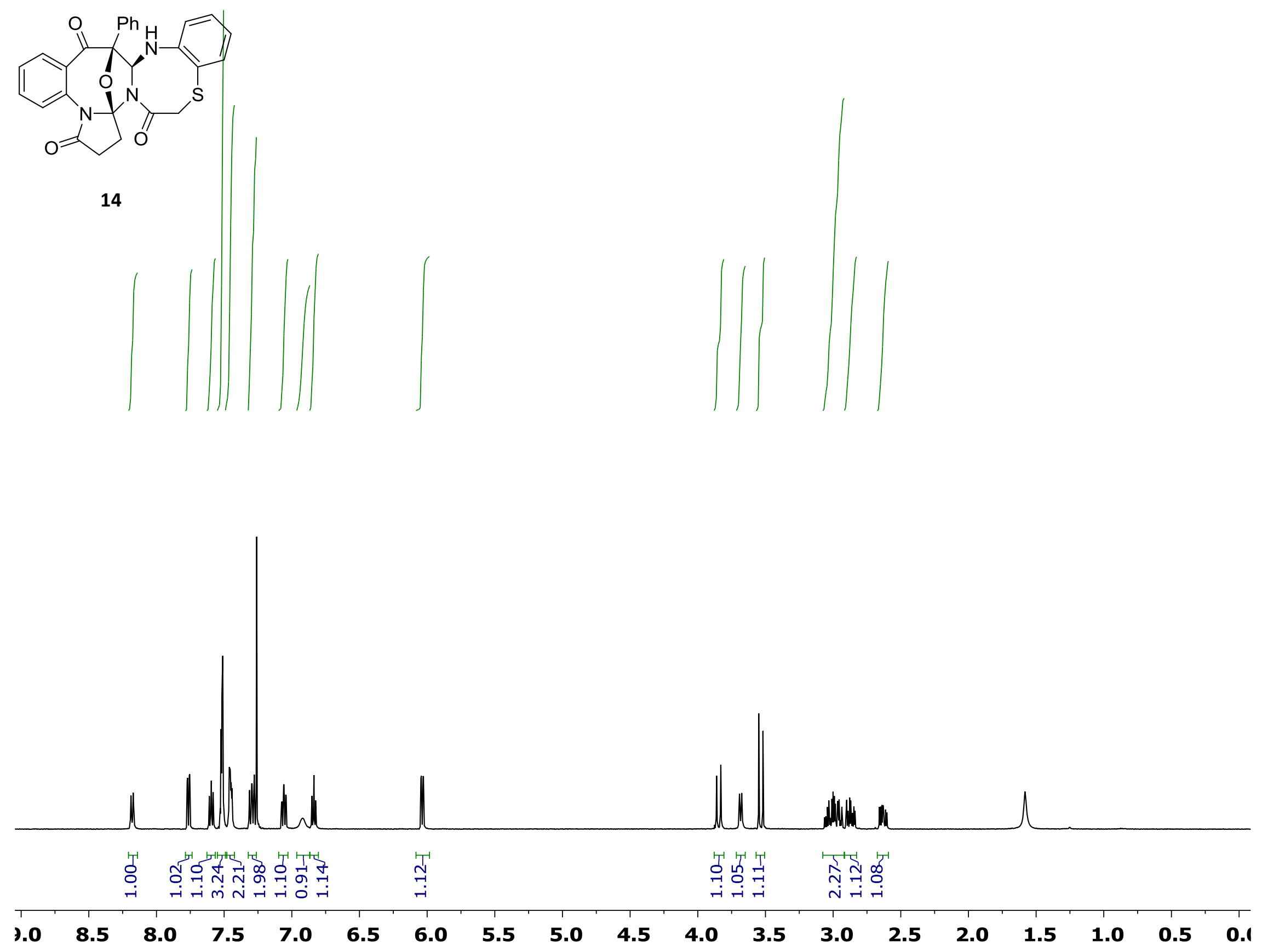


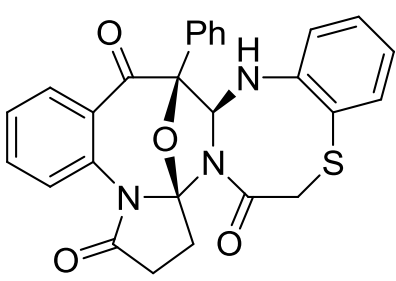

14

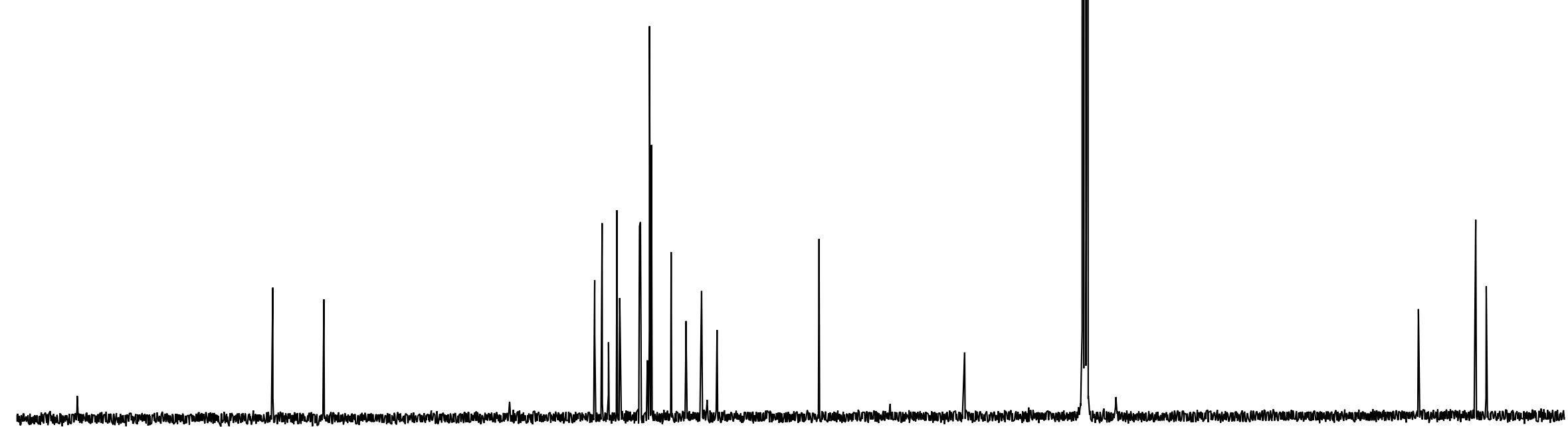




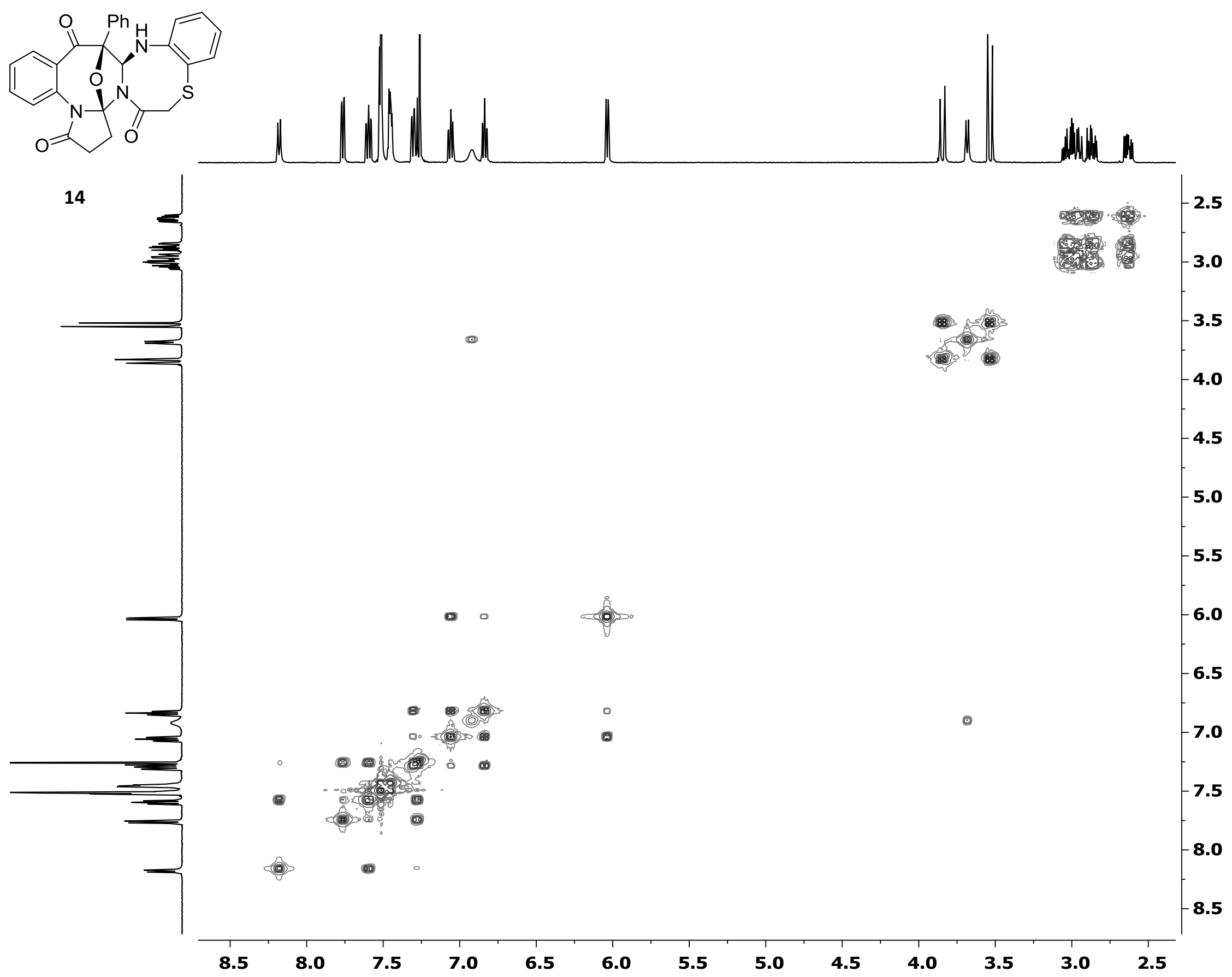




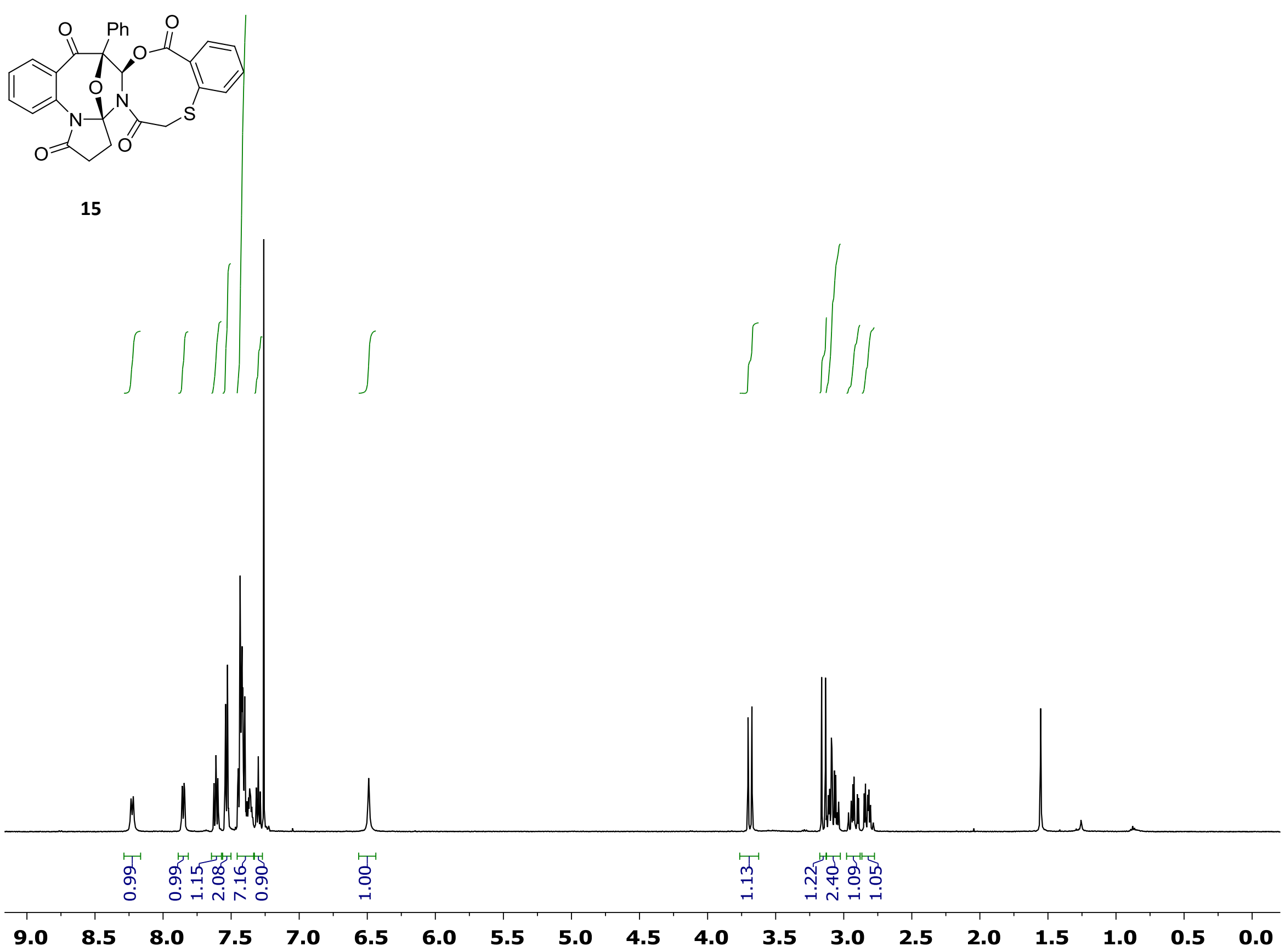




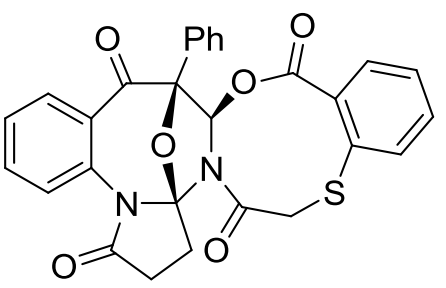

15

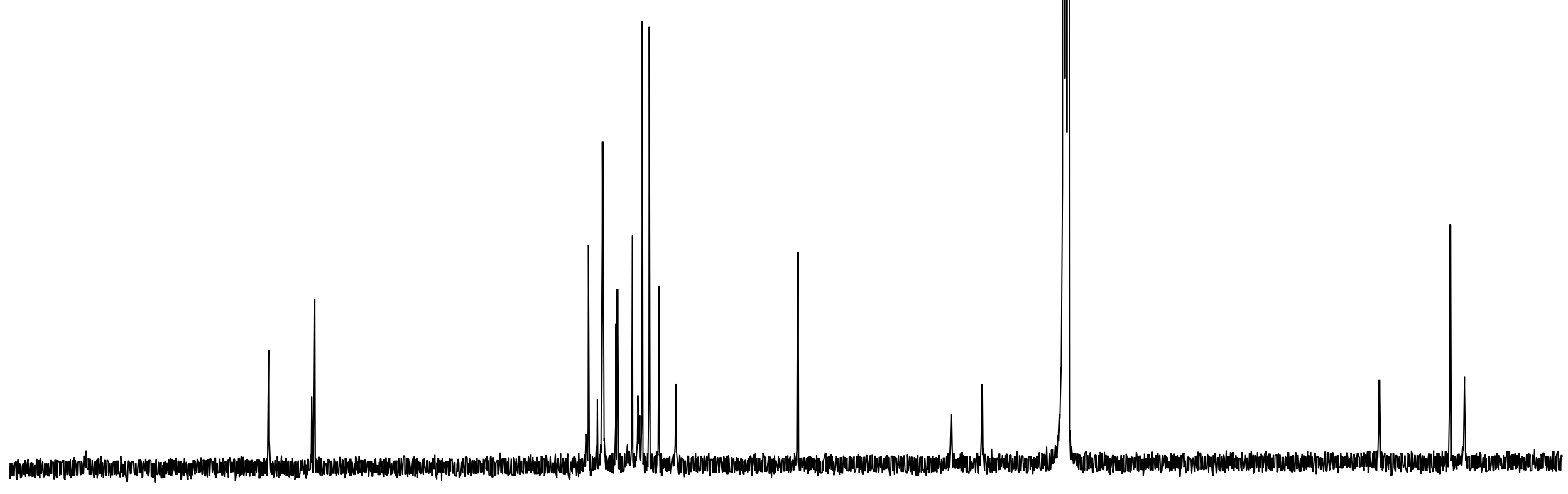




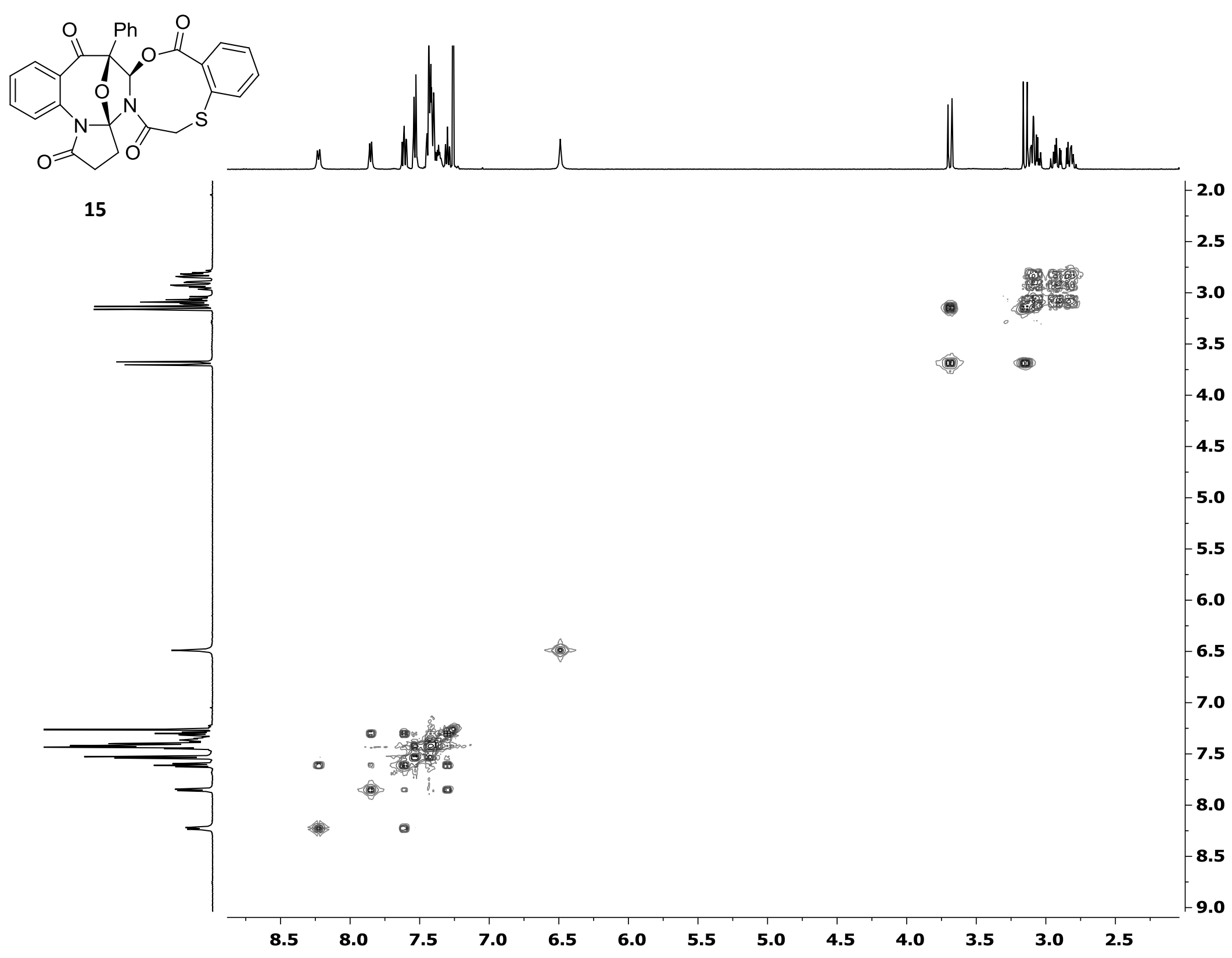

